\title{
THE CO-CONSTRUCTION OF ENTREPRENEURIAL SENSEMAKING
}

AN EMPIRICAL EXAMINATION

OF SOCIALLY SITUATED COGNITIVE MECHANISMS

IN ENTREPRENEURIAL COGNITIVE DEVELOPMENT

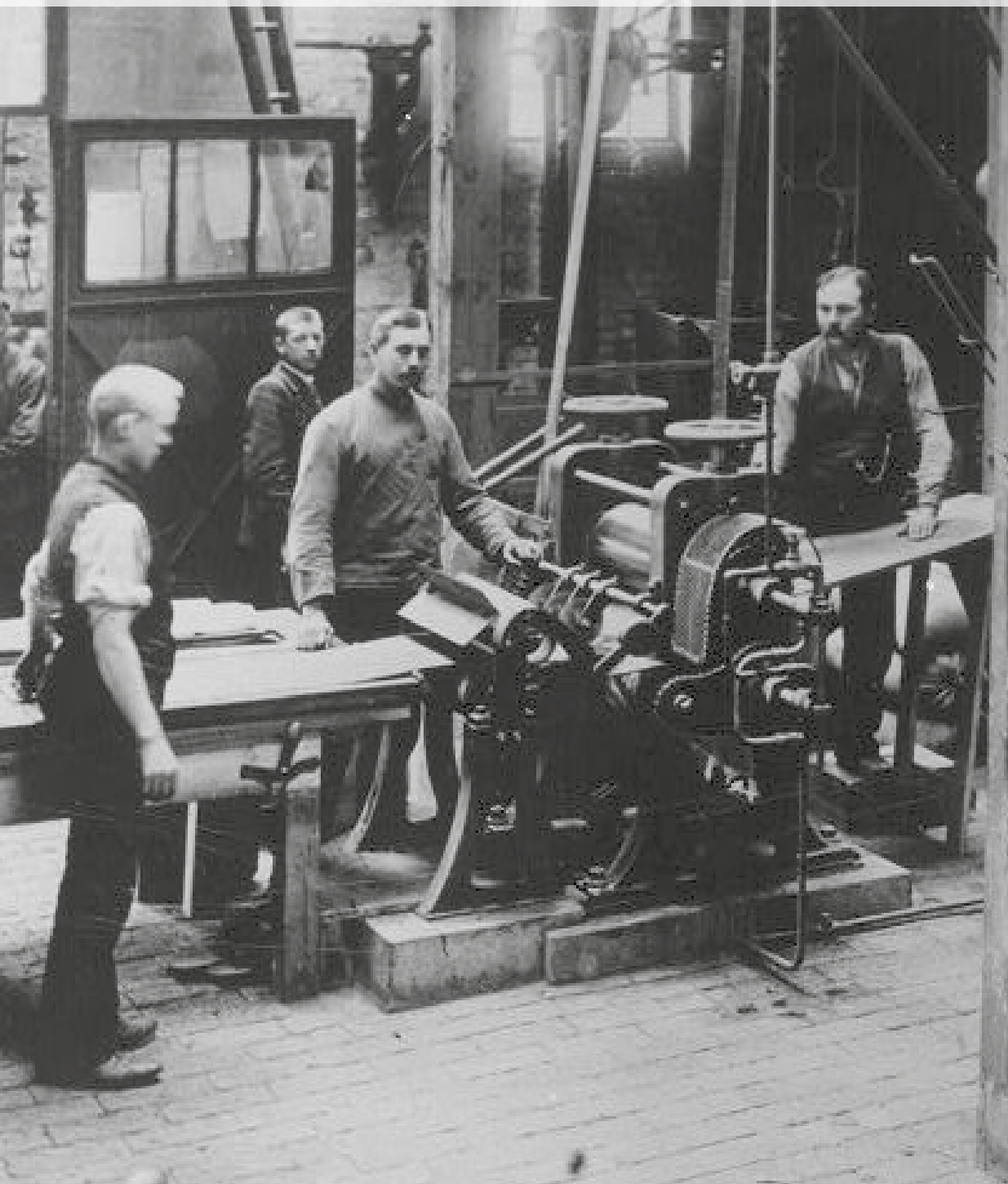

Gabi A. Kaffka 



\title{
THE CO-CONSTRUCTION OF ENTREPRENEURIAL SENSEMAKING
}

\author{
AN EMPIRICAL EXAMINATION \\ OF SOCIALLY SITUATED COGNITIVE MECHANISMS \\ IN ENTREPRENEURIAL COGNITIVE DEVELOPMENT
}




\section{Graduation committee:}

Chairman/secretary: Prof. dr. T.J.A. Toonen University of Twente

Supervisors:

Prof. dr. A.J. Groen

University of Twente

Dr. ir. J. Kraaijenbrink

University of Twente

Members:

Prof. dr. P.C. de Weerd-Nederhof

Univeristy of Twente, BMS

Dr. M.L. Ehrenhard

University of Twente, BMS

Prof. dr. J. Kratzer

TU Berlin, Germany

Prof. dr. P. Englis-Danskin

Campbell School for

Business, Berry College,

USA

Prof. dr. B.G. Englis

Campbell School for

Business, Berry College, USA

Dr. N. Krueger

Entrepreneurship Northwest,

USA

Cover: Reinhold Lessmann. Grammophonfabrik der Brueder Joseph und Emil Berliner, Kniestrasse 18. Hannover-Nordstadt; original upload on Wikimedia on 12 July 2004; The Library of Congress, 101 Independence Ave, SE Washington, DC 20540

ISBN: 978-90-365-4320-0

DOI: $10.3990 / 1.9789036543200$

(C) Gabi Kaffka, 2017

All rights reserved. No part of this publication may be reproduced in any form or by any means electronic or mechanical, including photocopying, recording or by any information storage and retrieval system, without permission in writing from the author. 


\title{
THE CO-CONSTRUCTION OF ENTREPRENEURIAL SENSEMAKING
}

\author{
AN EMPIRICAL EXAMINATION \\ OF SOCIALLY SITUATED COGNITIVE MECHANISMS \\ IN ENTREPRENEURIAL COGNITIVE DEVELOPMENT
}

\section{DISSERTATION}

to obtain

the degree of doctor at the University of Twente under the authority of the Rector Magnificus

Prof. dr. T.T.M. Palstra

on the account of the decision of the graduation committee to be publicly defended

on Wednesday May $24^{\text {th }} 2017$ at 12.45 hrs

by

\section{Gabi Anja Kaffka}

born on $29^{\text {th }}$ of May, 1981

in Hannover, Germany 
This dissertation has been approved by:

Prof. dr. A.J. Groen (supervisor)

Dr. ir. J. Kraaijenbrink (co-supervisor) 


\section{Foreword}

The cover of this dissertation shows men working at the company of Berliner's plant in Hanover, Germany. Throughout his life Emile Berliner, the inventor of the gramophone, was (sometimes painfully) aware of the role of other parties in realizing his value proposition - even in internationally context, which at the time was still unusual. The example of Berliner not only illustrates the benefits of co-creation of value and the implicit co-construction of sensemaking that goes with it. He also originates from the city I was born in.

The picture on the cover, with the men standing proudly at their industrial work place, signals the will to get things done - and the role of others in that difficult process of starting and running a business. In fact, starting a business never is easy. And I don't think entrepreneurs think that either. But how do they make sense of their business opportunity - since, after all, meaning is not 'just there'? That is the funny thing about meaning; it can be given willfully, intentionally, and directionally, but it will invariably also be shaped by others. It is this topic of co-construction of entrepreneurial sensemaking which fascinates me and which now, after almost seven years since my research began, has led to the writing of this book.

Ironically enough, the topic of my research made me increasingly aware of the fact that the execution of a dissertation itself involves the co-construction of sensemaking. Research questions are formulated, approaches decided and theoretical perspective- and all these activities involve other people who shape that process. And those people who affected my sensemaking I want to pay tribute to which is more than due.

There are my supervisors Aard Groen and Jeroen Kraaijenbrink. I want to thank both of them for their support, their patience, and their tolerance for my sometimes wildly random research pursuits. You gave me a lot of freedom to explore and experiment, and I am very grateful for that. Norris Krueger, you opened a world of intellectual adventure and freedom for me. Your brilliance is only equaled by your collegiality and good-spirited nature. I am serious when I say I owe you deeply, and I thank you for all that you've taught me and made possible. 
In terms of technical support at the University of Twente, none of this thesis would have been possible without the patient and professional support of Jan Jaap Struis, my personal ICT coach, who made NVivo run work time and again on my laptop.. hup hup JJ! Also a great thanks to the secretaries Monique and Joyce who always remained cool, calm, collected and sweet with my hundred and one questions.. You are a pleasure to have in the office! And in particular Joyce, whom I saw become an entrepreneur, thank you for our conversations and laughter.

There are also my colleagues who have made my time as a doctoral student at the University of Twente enjoyable and interesting. Raymond, you became an unexpectedly dear sparring partner, and I hope that once in a while we'll have opportunity for thought exchange in the future because it was not only work-functional but also a lot of fun. Michel, Björn and Sandor, your intellectual, business and political endeavors are inspiring. You three were like 'caring big brothers' for the 'younger' generation of Ph.D.'s, and I want to thank you for that. To dixit generation of Ph.D's - Arjan, Martin, Ellen, Raja and Natalie - I can only say: it was the greatest of fun with you, and thank you for the get-togethers and enjoyable evenings we had in Enschede and at your homes. It was truly an honor to work alongside you. Ellen, we all missed you after you chose to pursue your way outside the UT. You were a focal point in this PhD network and I am so grateful to have met you. Martin, the pub-quizzes with you will never be forgotten - thank you for never a dull moment! A special thanks goes to Raja and Natalie - there was something irresistible about our first year as Ph.D's, and although we drifted apart as our research topics started taking us to different areas of investigation, there will always be a very special place in my heart for the ellipsis as we knew it. Nathalie, chiccaa, you not only were a fantastic colleague, but became one of my best friends - thank you for all our 'shizzle' and good times!

A special thanks also goes to all my long-term friends who each have affected the sensemaking of myself throughout the years and also of this dissertation. Your friendship and support are invaluable for me. I will only mention the inner circle - though there are more who are extremely meaningful to me (and I particularly think of the best of all host sisters Camille and Laura). Daniel, thank you for your optimism and brotherly 
watchfulness. Petra, thank you for providing a little piece of Germany in Amsterdam and all those years of our friendship. Marianne en Juun, thank you for your friendship - in particular Marianne whose sweet and noble character is beyond belief - and I sincerely wish that IJburg forever will be the place where I can find you, good conversations, and great food - it's been a sanctuary for me. Philip - you know this is where the cow flies and people leave their cars to secure the street. We've been close for so long that it's almost we're like Statler and Waldorf from the Muppets, only much, much more fun of course. Michael, without our phone conversations and shared suspicion of heat this world would be a much less joyful place for me. I know I should not shower you with admiration, but you are one of the intellectual stars of my life, and I thank you for your support and critical encouragement throughout my life.

Charity and Onne, while I don't see or talk with you two as much as I would like, you have been very important to me throughout these years. Your friendship and the memories we share are priceless. It is those memories that I draw strength from and that give me energy. I wish we could live much closer to each other, so that I could enjoy your presence more often. Know that you are deeply appreciated. And Rebecca - almost 25 years of friendship make me fall short of words to describe what you mean to me. It is wonderful to have you and your family in my life, and I am endlessly grateful for all your advice, comfort, time together and of course Remo's and your hospitality and soul food.

To my sister Judith I wish to extend my appreciation for always being so patient with me being away and abroad. I know that I am not the best and closest of sisters, but your love has always been a warm reassurance of what family means, and I want to thank you for it. Also I thank my nephew Max who has taught me about what really matters in life.

And of course, first and foremost of all, the deepest appreciation for my parents, Bärbel and Herbert Kaffka. Without you two, none of this would have been possible literally, considering you are my parents. But even more so it was your love, encouragement and unconditional belief in me that made me the person I am as well as the author of this dissertation. And that is why, with utmost love, gratitude and admiration, I dedicate this dissertation to you. 

"La moindre chose contient un peu d'inconnu. Trouvons-le!" (Guy de Maupassant)

To my parents, Bärbel and Herbert Kaffka - für alles. 


\section{Table of Contents}

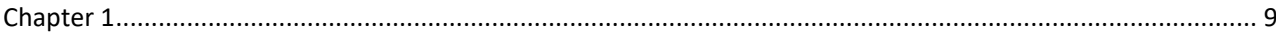

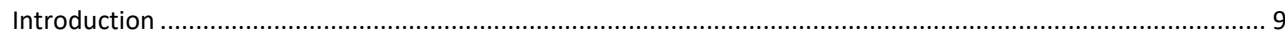

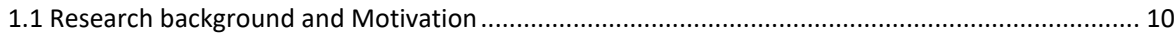

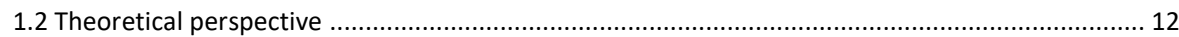

Defining Entrepreneurial Opportunity Development .................................................................... 12

The Role of Stakeholders in Entrepreneurial Cognitive Development ........................................... 13

Entrepreneurship as socially situated cognitive phenomenon .................................................... 14

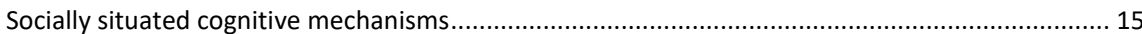

Studying socially situated cognitive mechanisms: the role of sensemaking .................................... 16

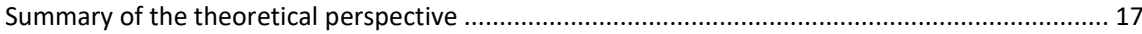

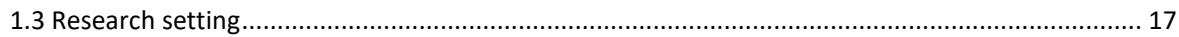

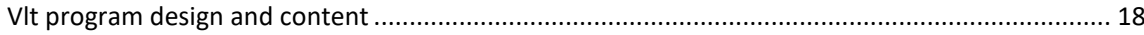

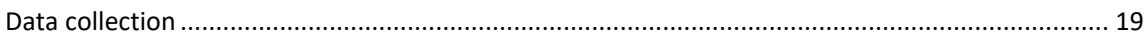

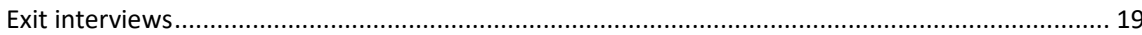

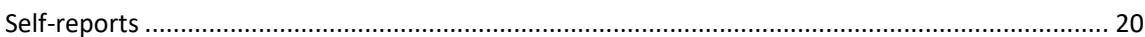

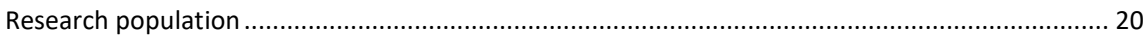

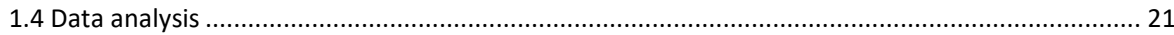

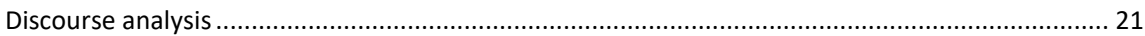

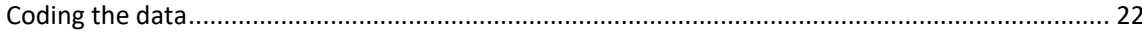

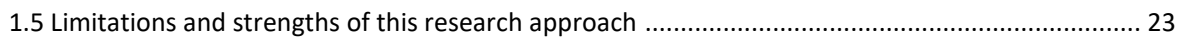

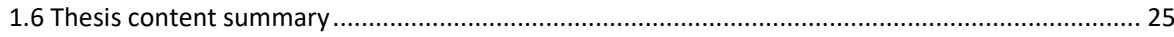

First study: effect of feedback mechanisms on entrepreneurial cognitive development ................. 26

Second study: sensebreaking mechanisms in entrepreneurial cognitive development................... 27

Third study: Mental maps and the development of a market orientation ...................................... 27

Fourth study: Sensemaking of the business model artifact.......................................................... 28

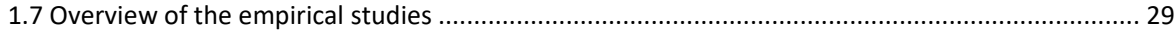

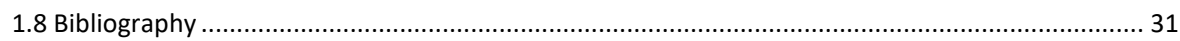

Chapter 2 
Cowboys, Greenhorns, Trappers and Saloon-owners: A 'Wild West' Taxonomy of Targeted Feedback Effects

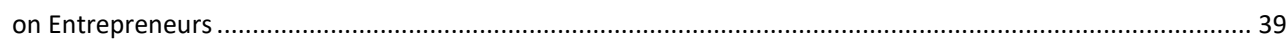

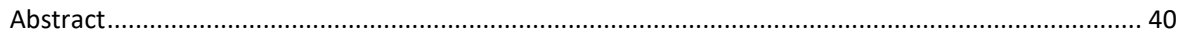

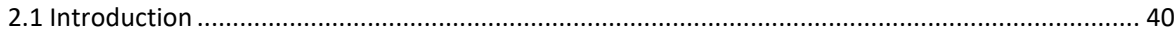

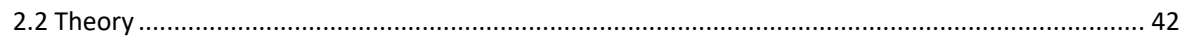

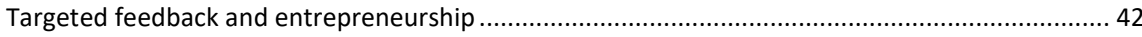

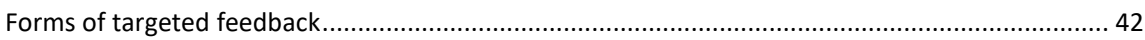

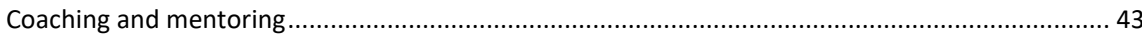

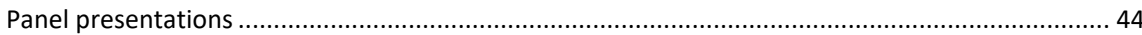

Targeted feedback and cognitive development ................................................................ 45

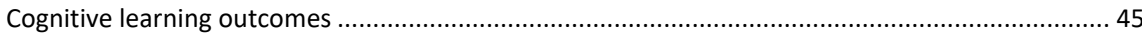

Taxonomy development based on the effects of targeted feedback mechanisms ........................ 46

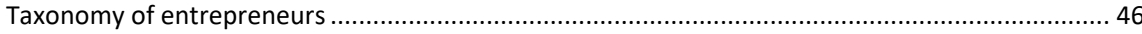

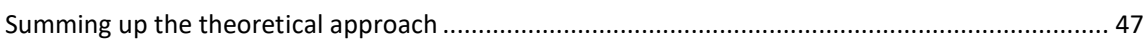

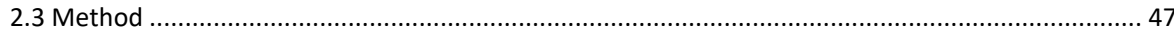

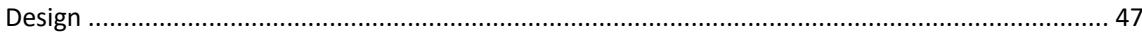

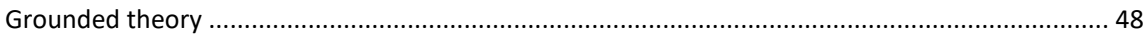

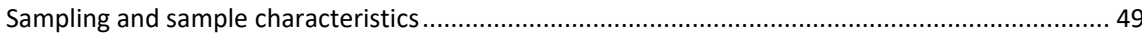

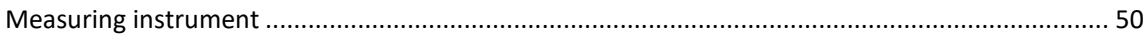

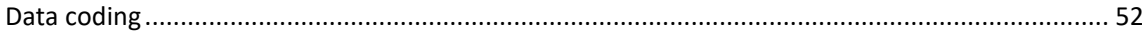

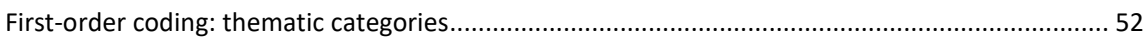

Second-order coding: cognitive learning outcomes ............................................................. 53

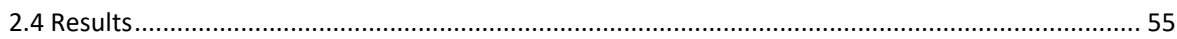

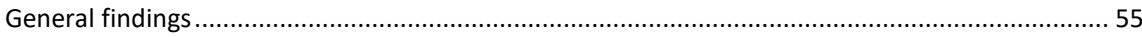

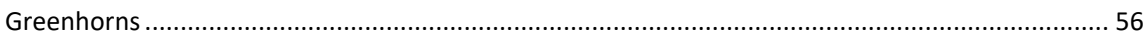

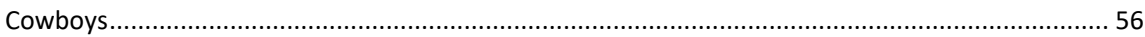

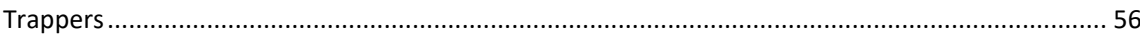

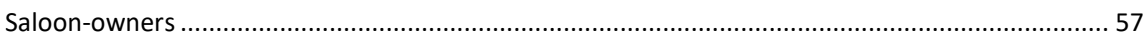

Type 1: Greenhorns - Novice entrepreneurs developing a service-based value offer ..................63

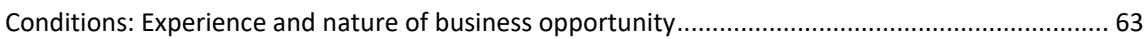

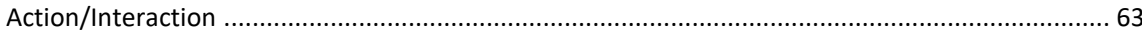


Type 2: Cowboys - Novice entrepreneurs developing a product-based value offer.....

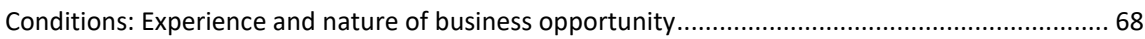

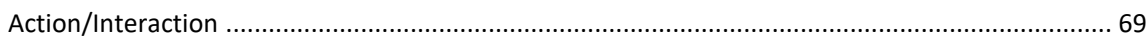

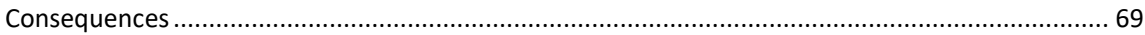

Type 3: Trappers - Experienced entrepreneurs moving from service-based value offer to productbased value offer

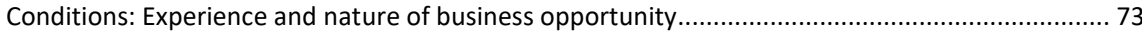

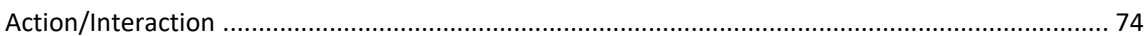

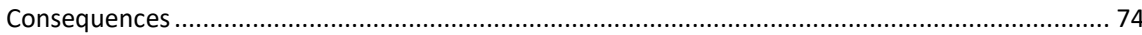

Type 4: Saloon-owners - Experienced entrepreneurs expanding an existing product-based value offer

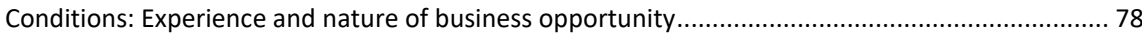

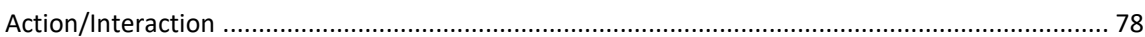

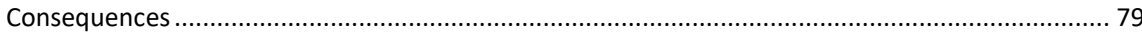

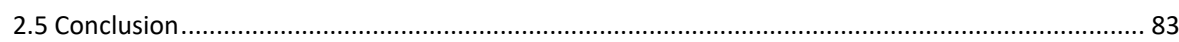

Taxonomy of entrepreneurs: how entrepreneurial experience and type of business opportunity

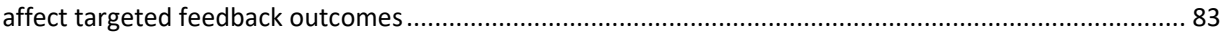

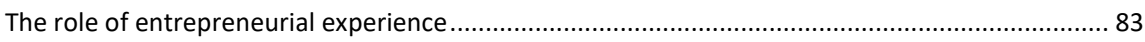

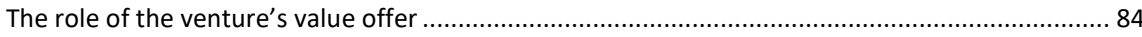

Comparing the effects of coaching and panel feedback on cognitive development of

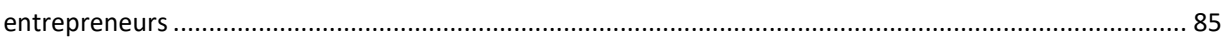

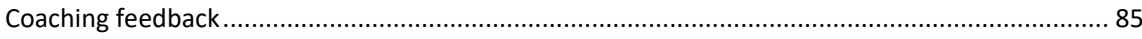

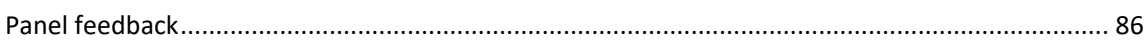

Outcomes of targeted feedback mechanisms: similarities and contrasts ................................... 86

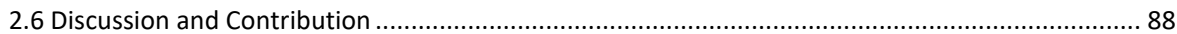

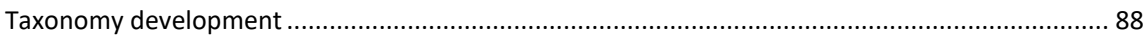

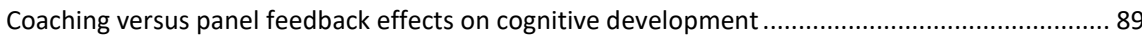

Targeted feedback mechanisms as forms of socially situated cognition ...................................... 90

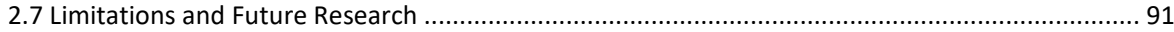

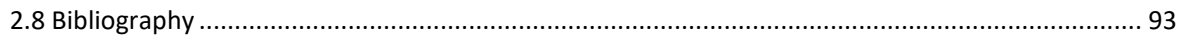




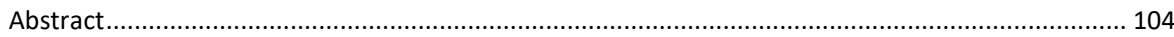

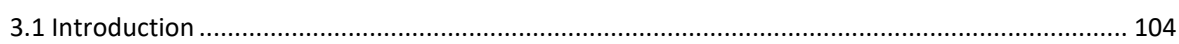

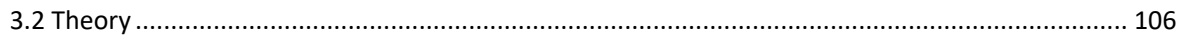

Sensemaking as a socially situated cognitive phenomenon ................................................ 107

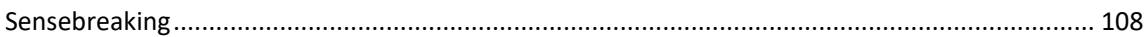

Effect of sensebreaking on entrepreneurial cognition: novel sensemaking of resources .............. 110

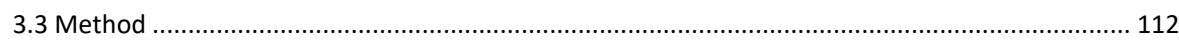

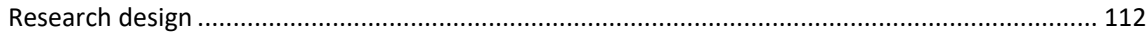

Data collection instrument ............................................................................................... 112

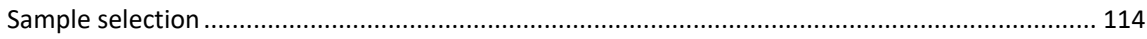

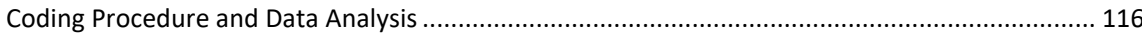

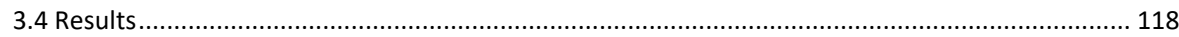

Differences in frequency of sensebreaking dimensions .................................................... 119

Differences in stakeholders involved in sensebreaking .......................................................... 120

Sensebreaking and its effects on entrepreneurial cognition ............................................ 121

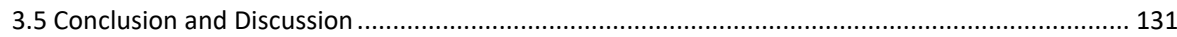

Differential effects and frequencies of sensebreaking mechanisms on entrepreneurial cognitive

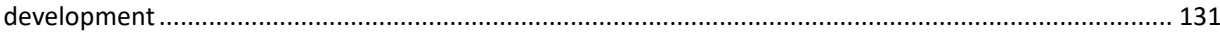

Effects of different stakeholders on sensebreaking ......................................................... 133

The concept of sensebreaking in the context of socially situated cognition ............................. 135

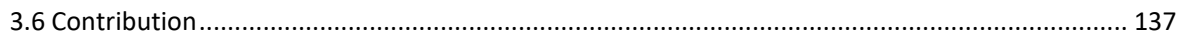

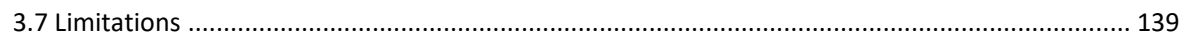

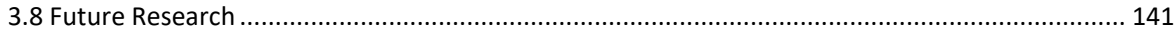

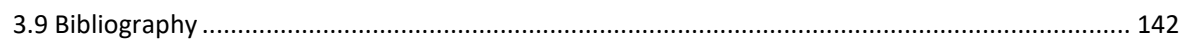

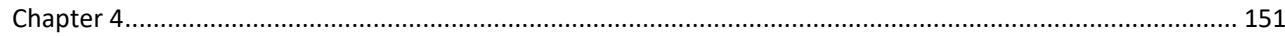

Sensemaking beyond the customer: A cognitive perspective on market orientation development ............... 151

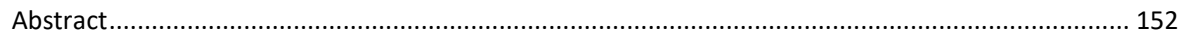

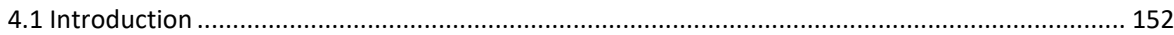

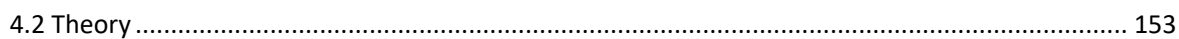

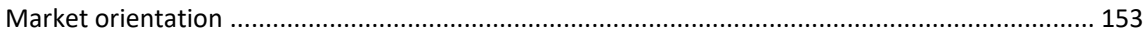

The development of a market orientation from a cognitive perspective................................... 154 
Sensemaking as market-oriented problem solving ........................................................... 157

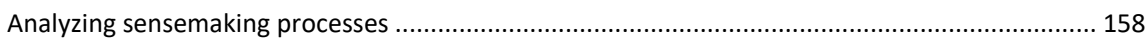

Market-oriented problem solving as categorization ........................................................... 159

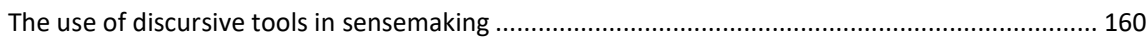

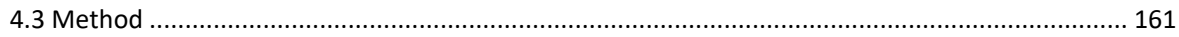

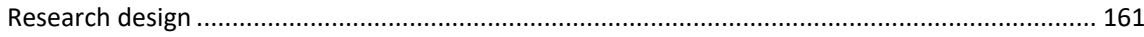

Qualitative research ............................................................................................................ 161

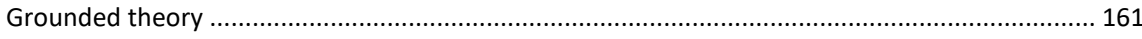

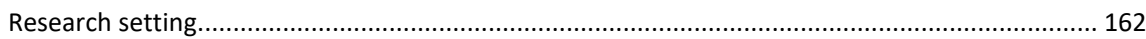

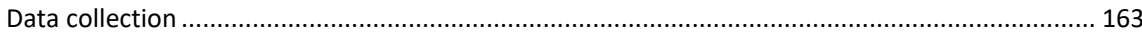

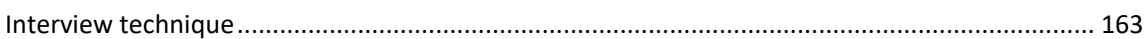

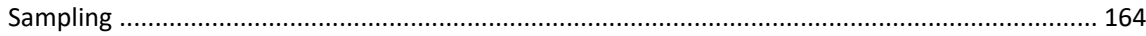

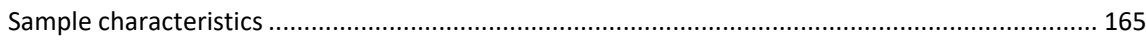

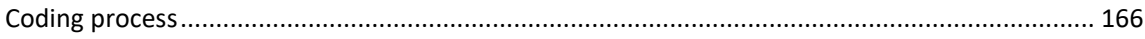

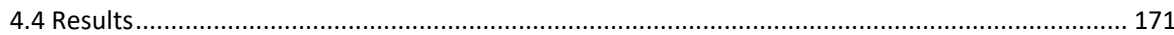

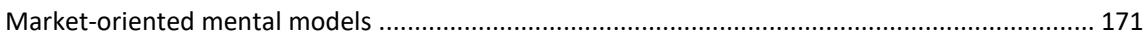

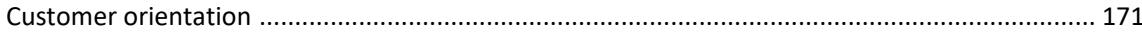

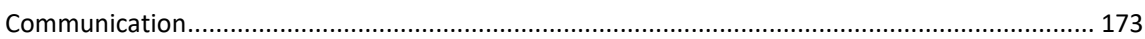

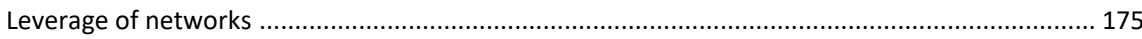

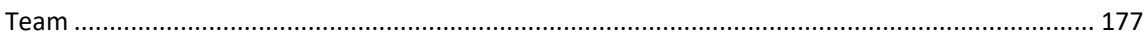

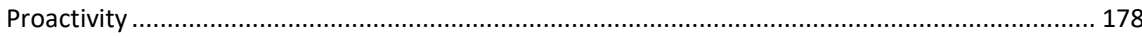

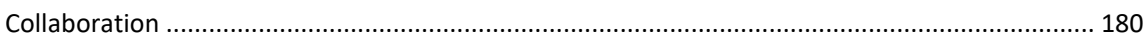

Comparing market-oriented mental models of novice and experienced entrepreneurs.............. 181

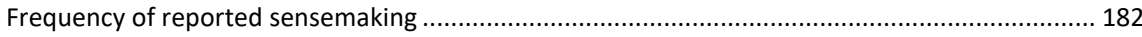

Content of market-oriented mental models.................................................................... 183

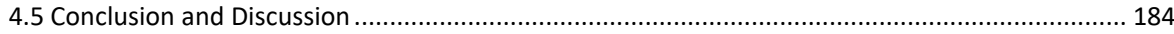

Market-oriented mental models and existing market orientation theory ................................... 185 
The difference between novice and experienced entrepreneurs: From mental models to mental schemas

4.6 Contribution 190

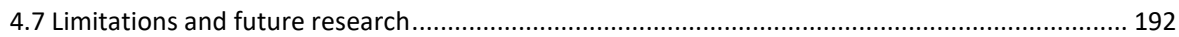

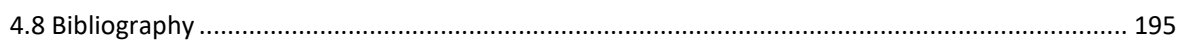

Chapter 5 203

Making sense of the business model: A cognitive investigation into the use of the business model artifact by entrepreneurs

Abstract 204

5.1 Introduction 204

5.2 Theory. 206

The business model artifact in the literature. 206

Sensemaking of the business model artifact 208

Purposes of the business model artifact. 209

The role of education in opportunity development 215

5.3 Method 216

Research design . 216

Setting 216

Measuring education

Data collection

Biased questioning and answering: The role of the 'Question under Discussion' and social desirability

Sample characteristics 220

Data analysis

Coding process

Inter-rater reliability

5.4 Results.

Comparison of the sub-samples by means of a t-test

Purposes reported by entrepreneurs

The purpose of understanding the venture's value creation logic 230

The purpose of developing the venture's value creation logic. 232

The purpose of communicating about the venture's value creation logic 233 
The role of education in business model use: differences between sensemaking of stem and nonstem entrepreneurs

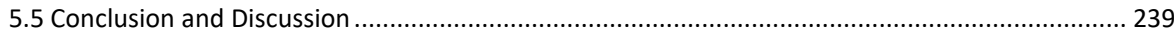

Sensemaking of the business model artifact purposes ........................................................... 240

The business model artifact as socially situated cognitive mechanism .................................. 241

Practical implications of this study .................................................................................... 242

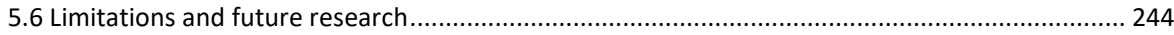

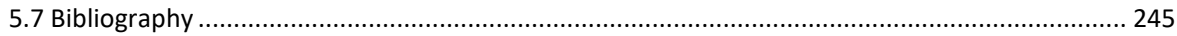

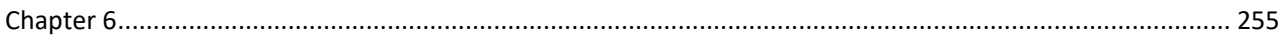

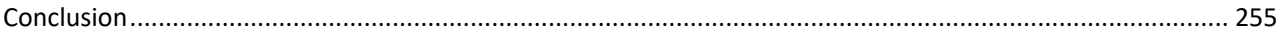

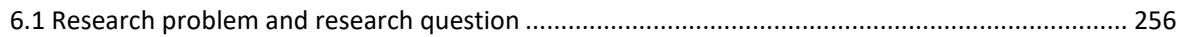

Summary of Chapter 2: Wild west: Targeted feedback mechanisms and their effect on cognitive

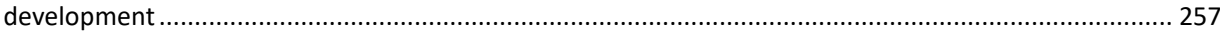

Summary of Chapter 3: Sensebreaking and development of entrepreneurial cognition ............... 258

Summary of Chapter 4: Mental models of market orientation .............................................. 259

Summary of Chapter 5: Sensemaking of the business model concept ...................................... 260

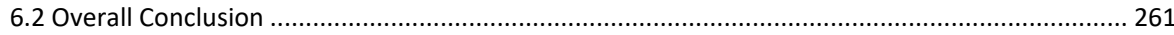

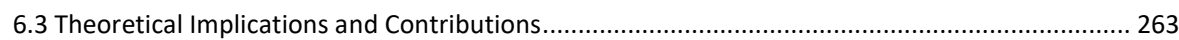

The contribution of the sensemaking perspective ........................................................................ 263

How socially situated cognitive mechanisms enhance learning about language ......................... 265

Methodical implications: Diary research ............................................................................. 266

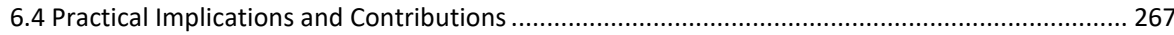

Implications for entrepreneurs ............................................................................................. 267

Implications for entrepreneurship education .................................................................. 268

Entrepreneurship support structures ............................................................................... 269

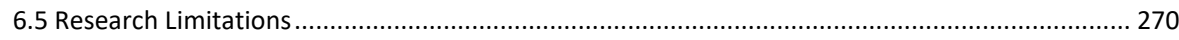

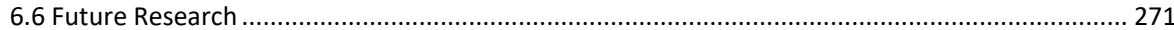

Self-reports: A future alley of data collection in entrepreneurship studies ................................ 271

Studying the explanatory role of socially situated cognitive mechanisms in entrepreneurial cognitive development 
6.7 Last words: The value of analyzing socially situated cognitive mechanisms .....

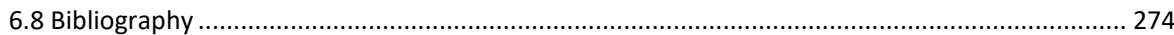

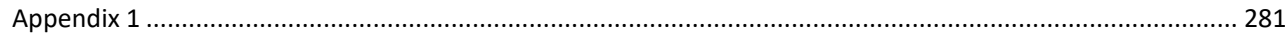

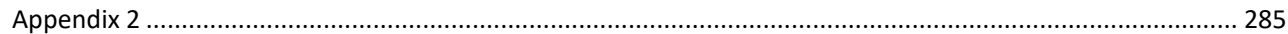

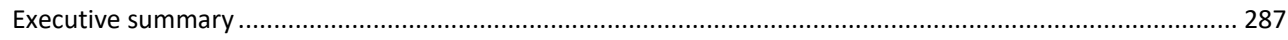

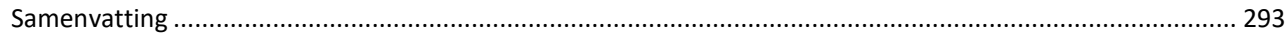


Chapter 1

INTRODUCTION 


\subsection{ReSEARCH BACKGROUND AND MOTIVATION}

Entrepreneurship contributes to innovation, firm creation, and employment generation (Ripsas, 1998; Shane \& Venkataraman, 2000) and therefore attracts a considerable amount of attention by academics and policy-makers (Shane, 2012). In particular, the last two decennia, cognitive aspects of entrepreneurial opportunity identification have received increasing attention by researchers (Baron, 1998; Mitchel et al., 2002; Grégoire, Corbett \& McMullen, 2011).

Entrepreneurial cognition refers to knowledge structures involved in decisionmaking and interpretation processes that influence the process of value creation in various ways (Grégoire et al, 2010). Cognitive theory gives consciousness "a fundamental role in human action" (Shaver \& Scott, 1991: p. 26). Examples of cognitive structures include heuristics and biases, mental maps and representations, metaphors, analogies or symbols that are used by entrepreneurs (Hill \& Levenhagen, 1995; Brännback \& Carsrud, 2009a, 2009b; Zott \& Huy, 2007; Bingham \& Eisenhardt, 2011; Clarke, 2011; Cornelissen et al., 2012).

Various studies have found that cognitive ability is related to the direction and performance of action (Autere \& Autio, 2000). This means that entrepreneurial cognition is not only related to mental processes, but that it is also inextricably intertwined with entrepreneurial action. The existing literature acknowledges the role of entrepreneurial cognition in business opportunity development; yet, empirical studies on this subject are scarce (Krueger, 2003; 2007; Bingham \& Eisenhardt, 2011). Specifically, we know little about the micro-level processes by which actors manage to influence the entrepreneur's cognitive development; how entrepreneurial cognition develops, or how it is expressed in different steps of the entrepreneurial process, remains therefore a relevant research question (Mitchell et al., 2007; Dew et al., 2015).

In particular, while the literature has recognized the social cognitive perspective of entrepreneurial opportunity development (Shepherd \& Krueger, 2002; West, 2007; Hmielski \& Baron, 2009; Mitchell et al., 2011) we lack qualitative insights into the social cognitive processes which affect the development of entrepreneurial cognition (Ozgen \& Baron, 2007) and the social cognitive mechanisms which facilitate entrepreneurial development. Social cognitive mechanisms are for example, observational learning, 
modeling and social referencing (White et al., 2014) and involve the interaction with or consideration for other individuals or groups. In recent years, an approach termed socially situated cognition has been introduced in entrepreneurship research.

Socially situated cognition integrates social psychology and situated cognition research and views entrepreneurship as a dynamic process in which cognitive, motivational, and emotional regulatory abilities of entrepreneurs interact together within specific social situations, with specific social actors (Mitchell et al., 2011).

This thesis focuses on socially situated cognitive mechanisms by which various parties affect the development of entrepreneurial cognition. Socially situated cognitive mechanisms are linked to social cognition and specifically focus on the action-oriented, situated, embodied and distributed nature of entrepreneurial cognition as described by Mitchell et al. (2011). The central research question which guides this dissertation is the following:

How do socially situated cognitive mechanisms affect entrepreneurial cognitive development during business opportunity development?

To answer this question, we undertook four empirical studies. These studies yield insights into the way that entrepreneurial cognition, in the form of sensemaking processes, is not only developed by the entrepreneur himself, but shaped in coconstruction with stakeholders and with the aid of artifacts. The first study focuses on targeted feedback mechanisms, in the form of panel and coaching interactions, while the second study analyzes sensebreaking mechanisms by which existing understandings are challenged by third parties which enables new sensemaking processes. The third study examines the development of market-oriented mental models among entrepreneurs; and the fourth study focuses on entrepreneurial sensemaking of using the business artifact concept during opportunity development.

The objective of this thesis is to provide empirical evidence to show how socially situated cognitive mechanisms affect the development of entrepreneurial cognition. Insights into the socially situated mechanisms of sensemaking co-construction yield valuable insights into the micro-foundations of interactive entrepreneurship which has been called for in the literature (Shepherd, 2015). More practically, knowledge about these mechanisms can be leveraged for venture start-up activities, by means of 
facilitating suitable business incubation or acceleration interventions. For example, the socially situated cognitive mechanism of sensebreaking we examine in Chapter 3 can be leveraged in order to stimulate the development of a (more) entrepreneurial mindset.

The rest of this chapter is built up as follows. First, an overview of the theoretical underpinnings of this thesis are presented. Next, we elaborate on the research setting and its suitability for the study of entrepreneurial cognitive development. Then we provide an overview of the four empirical studies that this thesis contains.

\subsection{THEORETICAL PERSPECTIVE}

\section{Defining Entrepreneurial Opportunity DeVelopment}

Entrepreneurship studies are concerned with the process of discovery, evaluation and exploitation of opportunities as well as people who are engaged in this process (Shane \& Venkataraman, 2000). There are three central questions in entrepreneurship research: a) why, when, and how opportunities come into existence; b) who discovers and exploits them, how and why; and c) what different modes of actions are used for the exploitation of entrepreneurial opportunities (Shane \& Venkataraman, 2000; 2001). This thesis focuses on the first of these questions - namely how opportunities come into existence.

Sarasvathy et al. (2010) distinguish between three views on the concept of entrepreneurial opportunity: opportunity recognition, opportunity discovery and opportunity creation. While there is no consensus on the matter, the view of 'opportunity creation' (in which the commercial success of an opportunity is seen to be dependent on mutual negotiation among stakeholders) fits with our focus on the social context, and therefore, the role and importance of stakeholders in opportunity development.

The 'opportunity creation' view relates to business opportunity development as described by Archidivili, Cardozo \& Ray (2003). These authors write that "entrepreneurs develop business opportunities to create and deliver value for stakeholders in prospective ventures. While elements of opportunities may be "recognized," opportunities are made, not found. [...] The need or resource "recognized" cannot become a viable business without this "development."” (Archidivili et al., 2003: p. 113). 
They add that the process of entrepreneurial opportunity development "is cyclical and iterative: an entrepreneur is likely to conduct evaluations several times at different stages of development; these evaluations could lead to recognition of additional opportunities or to adjustments to the initial vision." (Archidivili et al., 2003: p. 118).

In this thesis, we define business opportunity development as consisting of opportunity perception, discovery, creation as well as evaluation. Those opportunities are seen and analytically treated as being 'created' as described by Sarasvathy et al., (2010) - a view which supports a process-based, interactive perspective on opportunity development. Before we elaborate on the concept of socially situated cognition in entrepreneurship we will describe the concept of entrepreneurial cognition.

\section{The Role of Stakeholders in Entrepreneurial Cognitive Development}

Entrepreneurial cognition has been defined as "knowledge structures that people use to make assessments, judgments or decisions involving opportunity evaluation, venture creation and growth" (Mitchell et al., 2002: p.97). Entrepreneurs use previous knowledge to 'connect the dots'; for example they leverage mental models, metaphors and symbols in order to identify and successfully prepare a business opportunity (Hill \& Levenhagen, 1995; Baron \& Ensley, 2006; Zott \& Huy, 2007; Cornelissen et al., 2012). Cognitive processes are associated with mental representations of the self, of others, of events and contexts, and of other mental states and constructs (Grégoire et al., 2011). Cognitive differences at the individual level may determine how actors would execute entrepreneurial tasks (Forbes, 2005). For example, Palich \& Bagby (1995) found that entrepreneurs frame the same stimuli differently than other people which leads them to perceive a certain situation more positively.

External stakeholders are important for venture development because the acquisition of external (im)material resources is key to the venture's development, survival and growth. The literature on entrepreneurship has paid attention to the role of particular stakeholders such as the top management team (West, 2007) and groups consisting of founders and investors (Lim et al., 2013) who affect entrepreneurial cognition. Ozgen and Baron (2007) studied the role of mentors and other industry-related network actors in shaping entrepreneurial cognition and found that third parties such as mentors positively affect opportunity identification. Similarly, St-Jean \& Audet (2012) show that these parties offer varied types of support in addition to industry knowledge, 
in particular support in the entrepreneur's cognitive development. These studies show that third parties play an important role in entrepreneurial cognitive development.

\section{ENTREPRENEURSHIP AS SOCIALLY SITUATED COGNITIVE PHENOMENON}

Business opportunity development takes place in a social context and is affected by the entrepreneur's (inter)action with relevant stakeholders (Clarke \& Cornelissen, 2011). In fact, various authors have analyzed the creation of a business venture as a social activity and the role of social networks in entrepreneurship (Shaver \& Scott, 1991; Korsgaard, 2011; Leyden et al., 2013). Alvarez \& Barney (2007; 2013) describe entrepreneurial opportunities as 'social constructions' and enacted by entrepreneurs and stakeholder involved in value creation processes. West (2007), for example, points out how the socio-cognitive alignment of team members influences decision-making in entrepreneurial ventures - which can help to explicate how individual and organizational levels of analysis are related. In addition, Gemmell et al. (2012) found that shared cognition between entrepreneurs and trusted partners can help them generate creatively rich ideas.

Mitchell et al. (2011) proposed to study the phenomenon of entrepreneurship by using an approach termed 'socially situated cognition' (SSC). This approach combines social psychology and situated cognition research and facilitates the analysis of entrepreneurial cognition-in-action such as stakeholder interaction and their influence on the development of entrepreneurial cognition. SSC assumes that entrepreneurial opportunities are co-constructed by relevant stakeholders, such as investors or first customers (Clarke, 2011; Cornelissen \& Clarke, 2010). For example, Cornelissen \& Clarke (2010) have pointed to the importance of "embedding entrepreneurs in a social context and recognizing the role of that social environment in creating and justifying opportunities for ventures." (Cornelissen \& Clarke, 2010: 542).

SSC refers to feedback and information structures which shape entrepreneurial cognition and action. It consists of "[s]ensemaking through human-action-based language [which] may be conceived as a device that entrepreneurs use to direct and self-regulate their thinking processes in a social context (action oriented and embodied), a way that entrepreneurs may approach and in turn be influenced by a situated communication context (situated), and a tool entrepreneurs use to facilitate collective 
meaning and action in the developed or shared expertise vis-à-vis their new venture idea (distributed)." (Mitchell et al., 2011: pp. 4-5).

Various studies have analyzed entrepreneurial opportunity development using the socially situated cognitive approach. Some focus on distributed cognition in entrepreneurship and the way relevant stakeholders influence the sensemaking processes of entrepreneurs during the initial (and all following) economic value cocreation processes (Cornelissen \& Clarke, 2010; Dew et al, 2015). For example, West (2007) found that frames of references for managers/team members play an important role in what he calls the 'design of socio-cognitive grids', namely shared meaning about specific events or strategies to pursue. And Navis \& Glynn (2010) study the emergence of new market categories through legitimization towards others by means of people's meaningful action and find that linguistic frames, announcements and endorsements play an important role during entrepreneurial opportunity development. These meaningmaking processes are affected by the context and situation in which they take place and involve sensemaking processes by entrepreneurs.

The co-construction of sense - in other words, the development of meaning of previously unknown events and processes in collaboration with others - has been a focal assumption in SSC. Therefore, sensemaking plays a central role in socially situated cognition (Mitchell et al., 2011) and is seen as the result of intersubjective knowledge collaboration between stakeholders involved (Davidson, 2001; Mitchell et al., 2011; Venkataraman et al., 2012). SSC postulates that agency is 'emergent and distributed' across actors and that business opportunities are shaped by 'relational processes'. In other words, an entrepreneur's intentionality and other cognitive faculties as well as his behavior is shaped by and during the interaction of stakeholders involved in opportunity development (Garud \& Giuliani, 2013).

\section{SOCIALLY SITUATED COGNITIVE MECHANISMS}

Taking the perspective of SSC we conceptualize the development of entrepreneurial cognition as the co-construction of entrepreneurial sensemaking during business opportunity development. Specifically, we focus on socially situated cognitive mechanisms which affect the development of entrepreneurial cognition. The concept of mechanism links to some general ideas which are shared by most accepted definitions (Hedström \& Ylikoski, 2010): A mechanism is identified by the kind of effect or 
phenomenon it produces; it is an irreducibly causal notion and it has a structure; mechanisms form a hierarchy (lower level mechanisms explain higher level mechanisms) and mechanisms can be combined, e.g. two or more mechanisms can be combined to form a more complicated mechanism. The concept of mechanism has been applied in the social sciences to the study of social mechanisms, such as the relationship between education and social inequality (Boudon, 1974). And social-cognitive mechanisms have been analyzed in terms of their role in the interaction with, or consideration for, other individuals or groups; for example observational learning, modeling and social referencing (White et al., 2014).

In this study, we examine socially situated cognitive mechanisms by which stakeholders can affect entrepreneurial cognition, as well as those which are used by entrepreneurs to allow stakeholders to engage in the development of entrepreneurial cognition.

\section{STUDYING SOCIALLY SITUATED COGNITIVE MECHANISMS: THE ROLE OF SENSEMAKING}

Sensemaking consists of categorization (Weick, 1995; Weick et al., 2005). People construct and use mental categories to simplify and order the information they perceive. Categorization involves the "construction and bracketing of the textlike cues that are interpreted" by individual actors (Weick, 1995: p 12). Macrae \& Bodenhausen (2000) describe how people make sense of the social context and contribute to the construction of it by means of categorical thinking. Despite their importance in organizational contexts (Weick et al., 2005), sensemaking is a neglected topic in the field of entrepreneurship. Cornelissen (2013) for example suggests that we 'may need to dig deeper' into the role of language, interaction and thought in entrepreneurial sensemaking processes.

Sensemaking in the form of categorization activities is not only reflected in mental processes but also in language which is used for the expression of ideas and meaning. It has been argued that attention should be paid to "the dynamic and active interrelation between language and thought, labeled sensemaking, and [...] the important role of language as a key mediating mechanism or device in influencing the cognitions of others, including, say, investors and other prospective stakeholders of a venture." (Cornelissen \& Clarke, 2010: 542). The way we use language to express what we have done and imagine what we will do is a powerful instrument in shaping the business opportunity. 
It is through language that individual actors acquire knowledge about and from the (social) context in which they operate. This especially holds true in the case of business opportunity development which is a socially interactive process (Cornelissen \& Clarke, 2010). Language is therefore a focal point of analysis for the study of how shared meaning is created, and how third parties affect this. Clarke \& Cornelissen (2014) posit that language shapes entrepreneurial cognition by providing a means to verbalize one's ideas and "shape or limit the ways in which the speaker forms conceptions of the world" (Clarke \& Cornelissen, 2014: p. 387). For example, Cornelissen (2013) and Garud \& Giuliani (2013) point toward entrepreneurial narratives to understand entrepreneurship.

The role of language, in the form of entrepreneurial discourse which reflects categorization activities, is central to our study of socially situated cognition. The language used by entrepreneurs to describe the process of developing a business opportunity provides us with information about the perception and interpretation of entrepreneurs, and therefore also about their cognitive development.

\section{SUMMARY OF THE THEORETICAL PERSPECTIVE}

In sum, in this thesis we analyze the development of entrepreneurial cognition during business opportunity development, by means of examining different socially situated cognitive mechanisms which affect entrepreneurial sensemaking. Socially situated cognition emphasizes the role of language, in particular when analyzing entrepreneurial sensemaking. That is why we draw on data collected from entrepreneurs' discourse - in the form of verbal and self-written reports - about their engagement in business opportunity development. In order to ensure that the entrepreneurs are indeed engaged in opportunity development the research takes place in a setting of business incubation and acceleration.

\subsection{ReSEARCH SETTING}

The setting in which our research is carried out is a business incubation/acceleration program called the Venture Lab Twente. The Venture Lab Twente (VLT) is a business incubator/accelerator program and affiliated with the University of Twente (the Netherlands). It serves the purpose of regional economic stimulation, by means of helping to grow innovative, high-tech, small firms. The VLT 
program ran for four years, from 2010 to 2014 . This program was set up by multiple actors which include the European Union, Dutch provinces, regional governmental institutions as well as the University of Twente. The Dutch Institute of KnowledgeIntensive Entrepreneurship (NIKOS) located at the faculty of business administration of the University of Twente is in charge of organization and management of the VLT program.

The VLT program has gained an international reputation for its holistic and inclusive approach to entrepreneurship stimulation and support, and has received considerable (inter)national recognition for it. For example, the VLT won the national preliminaries European Enterprise Promotion Award 2013 and in the same year also won the Technopolicy Network Growth Award.

\section{VLT PROGRAM DESIGN AND CONTENT}

The VLT program offers a one-year trajectory to (aspiring) entrepreneurs who seek to develop a business opportunity. It is designed to incubate new ventures or accelerate the successful commercialization of novel products and services. Every three months a new cohort of participants entered the one-year trajectory, which meant that after the first full year of operation there was a constant influx of new participants replacing those finishing or leaving the program. Each cohort of the Venture Lab Twente consisted of up to 25 entrepreneurs.

Participant entrepreneurs can make use of the physical benefits of the VLT program; housing, access to office and associated ICT facilities. The trajectory also contains theory-based as well as practice-oriented trainings, intensive weekly coaching sessions, meetings with specialists (for example, fiscal experts), as well as access to networks and finance. Trainings take place at least one day a week, with an additional one hour of coaching per week, as well as tri-monthly panel presentations in which the entrepreneur receive feedback on their business idea. Also, other meetings are offered to participants, such as synergy and peer-to-peer feedback groups with participants of the program, and informal interaction between the entrepreneur and the VLT support staff. The high frequency for potential interaction with venture-relevant third parties in the VLT program is an intentional aspect of the VLT program design. In that way, the VLT program offers and catalyzes opportunities for interaction between entrepreneurs and 
stakeholders, providing favorable conditions for the analysis of socially situated cognition of entrepreneurs.

While the VLT targets novice high-tech, small firms it keeps its program admission flexible and therefore is open to entrepreneurs with various backgrounds. An integral part of the VLT program design is the data collection for research purposes and which furnished the data for this thesis.

\section{Data collection}

Various researchers of the University of Twente designed and implemented data collection points into the VLT program, with the aim of monitoring the development of the entrepreneurs and their ventures throughout the program. The goal of the data collection was not only to collect demographic and attributive data on entrepreneurs who develop a business opportunity - such as age, gender, education, self-efficacy, risk propensity, or other variables measured with quantitative scales - but also to monitor the development of the entrepreneurs and their opportunities throughout the program in a qualitative fashion. Therefore, the research and monitoring program consisted of quantitative and qualitative data.

The data used in this thesis is mainly drawn from two distinct qualitative data sets of the VLT business incubator program. These included in-depth interviews with entrepreneurs who exited the VLT program and self-reported logbook data which entrepreneurs provided during the VLT program in the form of a weekly diary. Below we describe both forms of data in more detail.

\section{EXIT INTERVIEWS}

Exit interviews took place with all entrepreneurs at the end of the one-year business incubation/acceleration program. The entrepreneurs were interviewed within three months of their leaving the program, in order to make sure that the reports that were collected reflected the entrepreneurs' recent impressions and perceptions and the maximum amount of details related to their thoughts and actions throughout the preceding year of business opportunity development. The interviews lasted between 40 minutes and two and a half hours. 
The following measures were taken to ensure the validity and reliability of the interview data. The questions asked in the interviews ensured a systematic collection of evidence and helped to avoid probing or 'fishing' for answers. Also, the structured nature facilitated the comparison of interview answers on a number of topics, such as the entrepreneurs' perception of the business model canvas. In Appendix 1 the complete interview blueprint can be found. In addition, the interviews were audio-taped and transcribed.

\section{SELF-REPORTS}

In addition to the interviews, the data set of this thesis stems from digital selfreports in the form of weekly diary or logbook entries which are collected via an online system. Those self-reports intended to capture a number of varied topics. They are structured along the following four open questions: 1) Learnings: What were the most important things that you learned in the past week? (2) Results: What results have you made in the past week? 3) Issues: What issues have you been most concerned with in the past week? and 4) Next Steps: What are the next steps that you are going to take in the coming weeks? These questions provide detailed data about the entrepreneur and the venture that was needed. The weekly diaries were collected digitally and allowed the documentation of the entrepreneur's own perception and interpretation of various, realtime, interactions during opportunity development.

The discourse collected via these two types of qualitative data provides the basis for the qualitative analysis of social-cognitive mechanisms which affect entrepreneurial sensemaking during opportunity development.

\section{RESEARCH POPULATION}

In total, more than 200 entrepreneurs participated in the VLT incubation/acceleration program. While the program focuses on high-tech startup ventures it does allow other applicants into the program, such as entrepreneurs of small, existing ventures with the plan to develop a new opportunity, or novice entrepreneurs without a high-tech idea. This means that the parameters of entry were less stringent and that the population of entrepreneurs who participated in the program was quite diverse. 
The average entrepreneur who participates in the VLT program is 44 years old (with a standard deviation of 10 years), has 14 years of work experience (here also with a standard deviation of 10 years) of as well as 7 years of entrepreneurial experience (standard deviation of 7 years). This shows that our sample consists of respondents with a diverse amount of both work and previous entrepreneurial experience. One out of eight participants is female which points to a strong over-representation of male entrepreneurs in our sample.

From the 200 entrepreneurs who participated in the VLT program, we selected those who are still pursuing the development of a business opportunity and of whom the exit interviews were taken by the time of our data collection. In addition, and in line with our focus on entrepreneurial cognitive development during opportunity development, we excluded exit interviews of entrepreneurs who had decided to abandon the development of an opportunity as well as family entrepreneurs and intrapreneurs (individuals who are not independently pursuing an opportunity but who exhibit entrepreneurial behavior within an already existing and established firm). Family entrepreneurs and intrapreneurs by definition operate in the context of organizational structures linked to an existing opportunity; this makes their business opportunity development process less relevant for the purposes of this study which focuses on novel opportunity development.

\subsection{Data ANALYSIS}

This thesis focuses on the role of socially situated cognitive mechanisms in entrepreneurial cognitive development. To study this phenomenon, we draw on qualitative data in order to analyze sensemaking processes which are reflected in the entrepreneurs' discourse.

\section{DISCOURSE ANALYSIS}

Discourse analysis is used for the examination of discourse content and meaning. It facilitates the understanding of how entrepreneurs perceive and interpret their actions and intentions and those of others by an in-depth focus on their justification and representations of those activities (Silverman, 2001). Discourse analysis is rooted in linguistics which refers to study of meaning conveyed in discourse. Meaning derives from the relations between individual elements of analysis - words - and systems of relations 
between words (Silverman, 2001). The root of linguistics lays in what Saussure (1916) describes as 'semiotics' (the study of origin and meaning of sign systems). As such, linguistics has concentrated mainly on historical changes in the meanings of words (Silverman, 2001).

Discourse analysis is more generally concerned with the interpretative repertoire, identities and category systems. In particular, categorization analysis (Silverman, 2001) is applied in the study of meaning construction. Categorization analysis is commonly applied in the study of sensemaking processes; it is associated with labeling activities undertaken by actors (Weick et al., 2005). Labeling or categorization activities play an important role in organizational sensemaking of strategic issues (Day \& Lord, 1992).

Discourse analysis fits well with our focus on entrepreneurial cognitive development for which we analyze sensemaking processes. Those sensemaking processes are contained in categorization activities manifesting in the entrepreneurs' discourse, and which also convey insights into processes by which socially situated cognitive mechanisms enable entrepreneurial cognitive development.

\section{CODING THE DATA}

A primary approach to coding in discourse analysis is to organize data into key categories of interests, themes and terms which arise from identification of similarities, variation, emphasis, and detail in the text (Seale, 1998). A point of attention in qualitative data coding is the validity and reliability of the data analysis and subsequent research results. These were ensured by audio-taping and transcription of the interviews, as well as digital collection of the self-reported logbooks of entrepreneurs. In this way, in our analysis the coders worked with low-inference descriptors: the descriptor data is available in verbatim and thus accessible for follow-up research and validation of all research results presented in this thesis. The coding results of each analysis are digitally stored in the NVivo (a qualitative data analysis program) and the Windows Excel (a spreadsheet application program for statistical and graphical purposes).

A codebook increased the reliability of the respective empirical study. The categories we identified are reported in code books aimed to eliminate ambiguity of meaning during the coding as much as possible. In the case of the deductive studies, those codebooks are informed by the operationalization of theoretical concepts, and help 
to make the coding 'logic' of the different coders more transparent and structured, and thus accountable. Also, researcher triangulation by independent reading and coding of the data by more than one researcher and subsequent comparison of coding results helped to safeguard the reliability of the research results.

The methodological aspects presented in sections $1.4-1.5$ are elaborated in more detail in the four empirical studies in Chapters 2 through 5.

\subsection{LIMITATIONS AND STRENGTHS OF THIS RESEARCH APPROACH}

There are a number of aspects which are a challenge in carrying out qualitative research. But there are also a number of reasons why a qualitative research method is appropriate and suitable for social scientific research in general, and in particular for answering the central research question of this thesis. In this section we first will elaborate on the limitations of this methodical approach, before turning to the arguments in favor for it.

A limitation of this thesis is that of the form of the data which regards the selfreported logbooks. The diary data is a very 'raw' form of data; the entrepreneurs were free to write down thoughts about their development, only guided by the four 'topics' within each weekly diary. The resulted in rather chaotic data; sometimes, sentences are not finished, various issues are mentioned in general without going into relevant details or names of the parties involved. With the exception of Kato and Wiklund (2011) who analyzed blog entries of entrepreneurs, insights into legitimate ways of doing logbook or diary studies are sparse. However, a clear advantage of this data collection method is its resulting real-time data which makes for a very pure and authentic form of research data. Also, the distinction between four topics in each diary entry facilitates a structured way of data collection.

A second limitation is found in the characteristics of our research sample. Our data was drawn exclusively from entrepreneurs in a business support environment, limiting it to entrepreneurs who are in need of (in)tangible resources in order to succeed, and who are therefore willing to participate in an institutional, theory-driven incubation/acceleration program. Therefore we are unable to draw general, population- 
wide conclusions about the phenomenon of entrepreneurial development. It can be noted however that the research sample consists of a diverse population of entrepreneurs - in terms of age, educational and industrial background and previous entrepreneurial experience - which assures that findings were not drawn on a homogeneous or biased group of entrepreneurs per se.

A third limitation of this study is the limitations in size of the research population. Since qualitative data analysis involves relative time-intensive data collection, manual coding and interpretative analysis it is often restricted in terms of the amount of respondents that can be processed. This is a constraint often encountered in qualitative research and yields the danger of anecdotic results and a lack of generalizability to a larger population. Certainly additional participants would make our results more robust. Yet qualitative research - and ensuing smaller samples than those that are able to be obtained with a quantitative research method - also makes for the richness of data we have collected and lays conceptual as well as methodological ground work for more large-scale, comprehensive studies in the field of entrepreneurship.

There are also distinct strengths about qualitative research methods which motivated our choice for its employment.

Firstly, with this research method we are able to come to understand how the process of 'meaning making' during opportunity development occurs, and specifically the role of third parties in this process. This is much needed in entrepreneurship where authors have called for the identification of cognitive drivers for entrepreneurial decisionmaking and action (Grégoire et al, 2010). The qualitative data used in this thesis yields insights into the interplay of entrepreneurial sensemaking processes and their actions, and is therefore suited for the identification of cognitive drivers, in the form of those entrepreneurial sensemaking processes.

Secondly, the collection of qualitative data enables us to avoid the bias of socially desirable responding (SDR). SDR is seen as a major pitfall in survey research (Bird, 2014). Qualitative data make it possible to ask open-ended questions and study the use of symbols, metaphors, denotations of words which reflect subjective impressions and individual sensemaking processes that are crucial to the study of entrepreneurial cognition as well as socially situated cognitive mechanisms which affect entrepreneurial cognition. Also, the collection of two types of qualitative data made it possible to cross- 
analyze the entrepreneurs' reports during the analysis, to check for corroborating or contractor reports by entrepreneurs on their own activities and thoughts.

Thirdly, the collection of two types of qualitative approach contributes methodologically, by providing a glimpse into the possibilities of different qualitative methods and their richness in terms of data. Specifically, this thesis combines real-time, longitudinal data in the form of weekly diaries with retrospective data in the form of indepth interviews which enable insights into entrepreneurial thought-processes over time which is beneficial in terms of data triangulation and thus contributes to the validity and reliability of the studies presented in this thesis.

Fourthly, by comprehensive qualitative data treatment, we can show "how the (theoretically defined) elements identified are assembled or mutually laminated" (Silverman, 2001: p. 290). SSC is a perspective intrinsically focusing on the time-related, embedded, distributed and interactive phenomenon of entrepreneurial opportunity development (Mitchell et al, 2011). SSC can therefore best be examined empirically, longitudinally and in-depth. We have done so by analyzing the reported perceptions and actions of entrepreneurs during business opportunity development.

A fifth argument in favor of qualitative research in general, and this thesis in particular, derives from the nature of the concept which is central to this study: sensemaking. Our choice for qualitative data provides us with the opportunity to "examine how particularly sayings and doings are embedded in particular patterns of social organization" (Silverman, 2001: p. 290) which is crucial to understanding entrepreneurial processes as Cornelissen \& Clarke (2010) have pointed out.

\subsection{THESIS CONTENT SUMMARY}

This first chapter contains the introduction of the study and a short overview of the empirical studies in the following chapters. The empirical studies that this thesis consists of and which are presented in Chapters $2-5$ each focus on a different socially situated cognitive mechanism or instrument of opportunity development. The results of those studies show that socially situated cognitive mechanisms offer ways for third parties to affect entrepreneurial cognition in interaction during opportunity development, but also 
offer tools for entrepreneurs to engage stakeholders in interaction which affects not only opportunity development but also entrepreneurial cognitive development.

The four studies provide empirical evidence of the enactive and interactive nature of entrepreneurial cognitive development, and contribute to our understanding of the social situatedness of that development. An overview of the four studies is given below.

\section{FIRST STUDY: EFFECT OF FEEDBACK MECHANISMS ON ENTREPRENEURIAL COGNITIVE DEVELOPMENT}

Chapter 2 consists of a study on the effect of different feedback mechanisms on entrepreneurial cognition during opportunity development. The research question of this study reveals how two different targeted feedback mechanisms - coaching and panel feedback - affect entrepreneurial cognitive development during opportunity development.

The literature has recognized the influence of targeted feedback on entrepreneurial cognitive development (St-Jean \& Audet, 2012). In this study we used the grounded theory approach and examine the effect of coaching and panel feedback on the cognitive development of entrepreneurs. To do so, we drew on longitudinal, real-time logbook data of 70 entrepreneurs inside a business incubation/acceleration program and we carry out a systematic comparison of the effects of those targeted feedback mechanisms.

We develop a taxonomy of entrepreneurs based on the type of value offer developed - product or service - as well as by prior entrepreneurial experience. The taxonomy yields four distinct types of entrepreneurs which we labeled 'Greenhorns', 'Cowboys', 'Trappers' and 'Saloon-owners'. Our findings provide empirical evidence of how the two feedback mechanisms affect the development of declarative knowledge, procedural knowledge and metacognition among entrepreneurs. With this study we contribute to theory-building on conceptualizing the role of different types of targeted feedback mechanisms in the development of entrepreneurial cognition of different categories of entrepreneurs. More practically, we provide insights into the ways in which institutional business support can optimally stimulate entrepreneurial cognitive development during opportunity development. 


\section{SECOND STUDY: SENSEBREAKING MECHANISMS IN ENTREPRENEURIAL COGNITIVE DEVELOPMENT}

Chapter 3 contains an empirical study on sensebreaking. In this study, we examine the effects of intersubjective sensebreaking on the development of entrepreneurial cognition and the contribution stakeholders provide.

Sensebreaking occurs when a person's existing understanding or process of sensemaking is disrupted by contradictory evidence. Sensebreaking challenges existing assumptions and thus creates a meaning void which is indispensable for novel sensemaking processes (Pratt, 2000). Sensebreaking instances induce entrepreneurs to reframe their interpretations of a situation, redirect their strategy or actions, and/or question the entrepreneur's individual learning and current understanding (Vlaar et al., 2008). These mechanisms of questioning, reframing and redirecting are central elements to our study of sensebreaking.

For this study we drew on qualitative data from weekly diary entries provided by entrepreneurs over a period of one year. In applying discourse data analysis we find that sensebreaking involves a range of stakeholders. Predominantly, sensebreaking is achieved by the mechanism of redirecting, followed by reframing and - much less frequent - questioning. The results show that sense-breaking enables novel sensemaking processes among entrepreneurs vis-à-vis a variety of topics relating to opportunity development. In particular, the reframing mechanism triggers sensebreaking in terms of metacognitive development.

We conclude that sensebreaking mechanisms of reframing, redirecting and questioning are useful for, and contribute to, a deeper understanding and conceptualization of critical feedback effects provided by different stakeholders on entrepreneurial sensemaking. Practically, the findings offer and provide insights into the functioning of sensebreaking mechanisms used inside and outside institutional business support settings.

\section{THIRD STUDY: MENTAL MAPS AND THE DEVELOPMENT OF A MARKET ORIENTATION}

The fourth chapter contains an empirical study led by the research question of how a market orientation develops among individual entrepreneurs. A market orientation is conceptualized in terms of mental models which reflect problem solving that is linked to the creation of (superior) customer value during business opportunity development. 
Taking a grounded theory approach, we analyze qualitative data from interviews with 50 entrepreneurs involved in business opportunity development and examine entrepreneurial problem solving regarding customer value creation, capture and delivery.

Our analysis yields a number of market-oriented mental models which entrepreneurs develop during opportunity development. Most notably, we find that market-oriented mental models predominantly link to a broader stakeholder orientation, and appear to shift from activity-related mental models - among novice entrepreneurs to more generic mental schemas used by experienced entrepreneurs.

With our findings we contribute to a better understanding of the cognitive development of a market orientation among entrepreneurs, seen as a relevant research and policy object (Shane, 2012). In particular, the results yield insights into the development of a market orientation and how it functions as mechanism for increasing entrepreneurs' awareness for stakeholders when solving problems in the creation of customer value, as well as encouraging interaction with those stakeholders. On a practical level, insights from this study can be used to optimize venture development in business support programs. More specifically, trainings geared towards the development of an entrepreneur's stakeholder orientation can be better designed.

\section{FOURTH STUDY: SENSEMAKING OF THE BUSINESS MODEL ARTIFACT}

The fifth chapter contains an empirical study which focuses on sensemaking by entrepreneurs of the business model artifact. The business model artifact as a concept can be used during business opportunity identification and development for different purposes.

We carried out a qualitative analysis of interviews with 85 entrepreneurs who are involved in business opportunity development, with particular attention paid to the effect of educational background on sensemaking processes regarding their use of the business model artifact. Entrepreneurs with a so-called 'STEM' background (an educational background in the academic disciplines of science, technology, engineering and mathematics) tend to focus on the technical aspects of their business idea (Berry, 1996). Therefore, we sought to examine the effect of a STEM background in the context of this study. The results show that entrepreneurs make sense of using the business model artifact mainly for understanding and developing their value creation logic. To a 
lesser extent, it is used for communicating and analytical purposes. Also, our findings show that STEM entrepreneurs use the business model artifact significantly more for communication and analytical purposes than non-STEM entrepreneurs.

The results contribute to the literature on entrepreneurship by demonstrating empirically, comparatively and systematically for which purposes the business model concept is used by entrepreneurs during opportunity development. In addition, our study contributes to theory-building on how human capital - in the form of education - affects sensemaking processes of the business model artifact during opportunity development. Practically our results encourage the more intensive use of the business model artifact's purposes of analysis and communication by entrepreneurs during business opportunity development.

\subsection{OVERVIEW OF THE EMPIRICAL STUDIES}

In Table 1-1 we present the individual research questions that guided each empirical study, its respective methodology, and the major results of each of the abovementioned studies. 


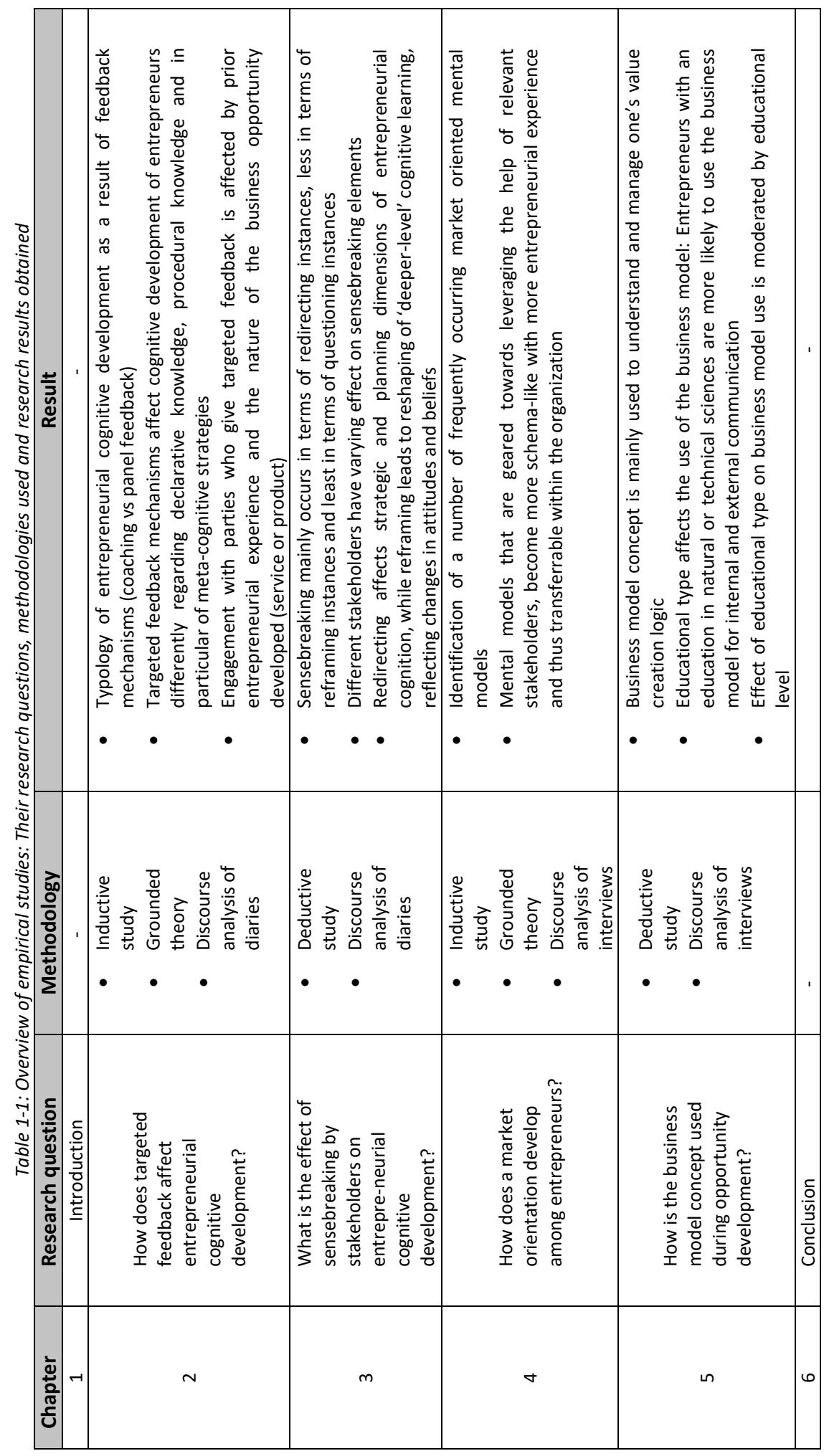




\subsection{BIBLIOGRAPHY}

Alvarez, S. A., \& Barney, J. B. (2007). Discovery and creation: Alternative theories of entrepreneurial action. Strategic entrepreneurship journal, 1(1-2), 11-26.

Alvarez, S. A., \& Barney, J. B. (2013). Epistemology, opportunities, and entrepreneurship: Comments on Venkataraman et al. (2012) and Shane (2012). Academy of Management Review, 38(1), 154-157.

Ardichvili, Alexander, Richard Cardozo, and Sourav Ray. "A theory of entrepreneurial opportunity identification and development." Journal of Business venturing 18.1 (2003): 105-123.

Autere, J., \& Autio, A. (2000). Is entrepreneurship learned? Influence of mental models on growth motivation, strategy, and growth. In Academy of Management Conference.

Bandura, A. (2001). Social cognitive theory: An agentic perspective. Annual review of psychology, 52(1), 1-26.

Baron, R. A. (1998). Cognitive mechanisms in entrepreneurship: Why and when enterpreneurs think differently than other people. Journal of Business venturing, 13(4), 275-294.

Baron, R. A., \& Ensley, M. D. (2006). Opportunity recognition as the detection of meaningful patterns: Evidence from comparisons of novice and experienced entrepreneurs. Management science, 52(9), 1331-1344.

Berry, M. M. (1996). Technical entrepreneurship, strategic awareness and corporate transformation in small high-tech firms. Technovation, 16(9), 487-522.

Bingham, C. B., \& Eisenhardt, K. M. (2011). Rational heuristics: the 'simple rules' that strategists learn from process experience. Strategic Management Journal, 32(13), 14371464. 
Bird, B. (2014). Toward a taxonomy of entrepreneurs' behavior. Handbook of Entrepreneurial Cognition, 113-131.

Boudon, R. (1974). Education, opportunity, and social inequality: Changing prospects in western society.

Brännback, M., \& Carsrud, A. (2009a). Cognitive maps in entrepreneurship: Researching sense making and action. In Understanding the entrepreneurial mind (pp. 75-96). Springer New York.

Brewer, M.B. \& Hewstone M. Social Cognition. 2004. Malden, MA: Blackwell Publishing Ltd.

Carsrud, A. L., \& Brännback, M. (Eds.). (2009b). Understanding the entrepreneurial mind: Opening the black box (Vol. 24). Springer Science \& Business Media.

Clarke, J. (2011). Revitalizing entrepreneurship: how visual symbols are used in entrepreneurial performances. Journal of Management Studies, 48(6), 1365-1391.

Clarke, J., \& Cornelissen, J. (2011). Language, communication, and socially situated cognition in entrepreneurship. Academy of Management Review, 36(4), 776-778.

Clarke, J. S., \& Cornelissen, J. P. (2014). 11. How language shapes thought: new vistas for entrepreneurship research. Handbook of Entrepreneurial Cognition, 383.

Cornelissen, J.P. and Clarke, J.S. (2010). Imagining and rationalizing opportunities: inductive reasoning, and the creation and justification of new ventures. Academy of Management Review, 35, pp. 539-557.

Cornelissen, J. P., Clarke, J. S., \& Cienki, A. (2012). Sensegiving in entrepreneurial contexts: The use of metaphors in speech and gesture to gain and sustain support for novel business ventures. International Small Business Journal, 30(3), 213-241.

Cornelissen, J.P. (2013). Portrait of an entrepreneur: Vincent van Gogh, Steve Jobs, and the entrepreneurial imagination. Academy of Management Review, 38, pp. 700-709. 
Davidson, D. (2001). Subjective, intersubjective, objective (Vol. 3). Oxford University Press.

Day, D. V., \& Lord, R. G. (1992). Expertise and problem categorization: the role of expert processing in organizational sense-making. Journal of Management Studies, 29(1): 3547.

Dew, N., Grichnik, D., Mayer-Haug, K., Read, S., \& Brinckmann, J. (2015). Situated Entrepreneurial Cognition. International Journal of Management Reviews, 17(2), 143164.

Fiske, S.T. \& Taylor, S.E. 2013. Social Cognition - From Brains to Culture. London: Sage Publications, Inc.

Forbes, D. P. (2005). Managerial determinants of decision speed in new ventures. Strategic Management Journal, 26(4), 355-366.

Garud, R., \& Giuliani, A. P. (2013). A narrative perspective on entrepreneurial opportunities. Academy of Management Review, 38(1), 157-160.

Gemmell, R. M., Boland, R. J., \& Kolb, D. A. (2012). The socio-cognitive dynamics of entrepreneurial ideation. Entrepreneurship Theory and Practice, 36(5), 1053-1073.

Grégoire, D. A., Barr, P. S., \& Shepherd, D. A. (2010). Cognitive processes of opportunity recognition: The role of structural alignment. Organization Science, 21(2), 413-431.

Grégoire, D. A., Corbett, A. C., \& McMullen, J. S. (2011). The cognitive perspective in entrepreneurship: An agenda for future research. Journal of Management Studies, 48(6), 1443-1477.

Hedström, P., \& Ylikoski, P. (2010). Causal mechanisms in the social sciences. Annual Review of Sociology, 36, 49-67. 
Hill, R. C., \& Levenhagen, M. (1995). Metaphors and mental models: Sensemaking and sensegiving in innovative and entrepreneurial activities. Journal of Management, 21(6), 1057-1074.

Kato, S., \& Wiklund, J. (2011). Doing Good To Feel Good-A Theory Of Entrepreneurial Action Based In Hedonic Psychology. Frontiers of Entrepreneurship Research, 31(4), 1.

Korsgaard, S. (2011). Entrepreneurship as translation: Understanding entrepreneurial opportunities through actor-network theory. Entrepreneurship \& Regional Development, 23(7-8), 661-680.

Krueger Jr, N. F. (2003). The cognitive psychology of entrepreneurship. In Handbook of entrepreneurship research (pp. 105-140). Springer US.

Krueger, N. F. (2007). What lies beneath? The experiential essence of entrepreneurial thinking. Entrepreneurship theory and practice, 31(1), 123-138.

Leyden, D. P., Link, A. N., \& Siegel, D. S. (2014). A theoretical analysis of the role of social networks in entrepreneurship. Research Policy, 43(7), 1157-1163.

Lim, J. Y. K., Busenitz, L. W., \& Chidambaram, L. (2013). New venture teams and the quality of business opportunities identified: Faultines between subgroups of founders and investors. Entrepreneurship Theory and Practice, 37(1), 47-67.

Macrae, C. N., \& Bodenhausen, G. V. (2000). Social cognition: Thinking categorically about others. Annual review of psychology, 51(1), 93-120.

Mitchell, R. K., Busenitz, L., Lant, T., McDougall, P. P., Morse, E. A., \& Smith, J. B. (2002). Toward a theory of entrepreneurial cognition: Rethinking the people side of entrepreneurship research. Entrepreneurship theory and practice, 27(2), 93-104.

Mitchell, R. K., Busenitz, L. W., Bird, B., Marie Gaglio, C., McMullen, J. S., Morse, E. A., \& Smith, J. B. (2007). The central question in entrepreneurial cognition research 2007. Entrepreneurship theory and practice, 31(1), 1-27. 
Mitchell, R. K., Randolph-Seng, B., \& Mitchell, J. R. (2011). Socially situated cognition: Imagining new opportunities for entrepreneurship research. Academy of Management Review, 36(4), 774-776.

Navis, C., \& Glynn, M. A. (2010). How new market categories emerge: Temporal dynamics of legitimacy, identity, and entrepreneurship in satellite radio, 19902005. Administrative Science Quarterly, 55(3), 439-471.

Ozgen, E., \& Baron, R. A. (2007). Social sources of information in opportunity recognition: Effects of mentors, industry networks, and professional forums. Journal of business venturing, 22(2), 174-192.

Palich, L. E., \& Bagby, D. R. (1995). Using cognitive theory to explain entrepreneurial risk-taking: Challenging conventional wisdom. Journal of business venturing, 10(6), 425438.

Pratt, M. G. (2000). The good, the bad, and the ambivalent: Managing identification among Amway distributors. Administrative Science Quarterly, 45(3), 456-493.

Santos, F. M., \& Eisenhardt, K. M. (2005). Organizational boundaries and theories of organization. Organization science, 16(5), 491-508.

Sarasvathy, S. D., Dew, N., Velamuri, S. R., \& Venkataraman, S. (2010). Three views of entrepreneurial opportunity. In Handbook of entrepreneurship research (pp. 77-96). Springer New York.

Saussure, F. D. (1916). 1966. Course in general linguistics. New York: McGraw Hill.

Seale, C. (1998). Qualitative interviewing. Researching society and culture. London: Sage, 202-216.

Seale. 1999. The quality of qualitative research. London: Sage.

Shane, S. (2012). Reflections on the 2010 AMR decade award: delivering on the promise of entrepreneurship as a field of research. Academy of Management Review, 37(1), 1020. 
Shane, S., \& Venkataraman, S. (2000). The promise of entrepreneurship as a field of research. Academy of management review, 25(1), 217-226.

Shane, S., \& Venkataraman, S. (2001). Entrepreneurship as a field of research: A response to Zahra and Dess, Singh, and Erikson. Academy of management review, 26(1), 13-16.

Shaver, K. G., \& Scott, L. R. (1991). Person, process, choice: The psychology of new venture creation. Entrepreneurship theory and practice, 16(2), 23-45.

Silverman, D. (2001). Interpreting qualitative data: Methods for analysing talk, text and interaction. London: Sage.

Smircich, L., \& Stubbart, C. 1985. Strategic management in an enacted world. Academy of Management Review,10: 724-736.

St-Jean, E., \& Audet, J. (2012). The role of mentoring in the learning development of the novice entrepreneur. International Entrepreneurship and Management Journal, 8(1), 119-140.

Strauss, A. \& Corbin, J. 2015. Basics of Qualitative Research. Newbury Park, CA: Sage Publications, Inc.

Venkataraman, S., Sarasvathy, S. D., Dew, N., \& Forster, W. R. (2012). Reflections on the 2010 AMR decade award: Whither the promise? Moving forward with entrepreneurship as a science of the artificial. Academy of Management Review, 37(1), 21-33.

Vlaar, P. W., van Fenema, P. C., \& Tiwari, V. (2008). Cocreating understanding and value in distributed work: How members of onsite and offshore vendor teams give, make, demand, and break sense. MIS quarterly, 32(2), 227-255.

Weick, K. E., Sutcliffe, K. M., \& Obstfeld, D. (2005). Organizing and the process of sensemaking. Organization science, 16(4), 409-421. 
Weick, K. E. (2012). Organized sensemaking: A commentary on processes of interpretive work. Human Relations, 65(1), 141-153.

West, G. P. (2007). Collective cognition: When entrepreneurial teams, not individuals, make decisions. Entrepreneurship Theory and Practice, 31(1), 77-102.

White, S. W., Mazefsky, C. A., Dichter, G. S., Chiu, P. H., Richey, J. A., \& Ollendick, T. H. (2014). Social-cognitive, physiological, and neural mechanisms underlying emotion regulation impairments: Understanding anxiety in autism spectrum disorder. International Journal of Developmental Neuroscience, 39, 22-36

Zott, C., \& Huy, Q. N. (2007). How entrepreneurs use symbolic management to acquire resources. Administrative Science Quarterly, 52(1), 70-105. 


\title{
CHAPTER 2
}

Cowboys, Greenhorns, Trappers and Saloon-oWners: A 'Wild West' Taxonomy of TARgeted Feedback Effects On ENTREPRENeURS

\author{
Gabi A. Kaffka, University of Twente \\ Norris Krueger, Entrepreneurship NorthWest \\ Jeroen Kraaijenbrink, University of Twente \\ Aard J. Groen, University of Twente
}

History of the manuscript: This chapter is a modified version of a peer-reviewed paper that was presented at BCERC conference 2014 in London, Ontario (Canada). 


\section{ABSTRACT}

In this study we examine the effect of different feedback mechanisms on entrepreneurs. We employ a grounded theory approach in our analysis of weekly logbooks of 70 entrepreneurs. Those entrepreneurs leverage two targeted feedback mechanisms - coaching and panel feedback - during participation in a one-year business incubation support program. We identify four categories of entrepreneurs - which we label greenhorns, cowboys, trappers and saloon-owners - that show distinctive uses and effects of different targeted feedback mechanisms. With this study, we contribute to theory-building by presenting a taxonomy of feedback effects in entrepreneurship, as well as practical evidence of the benefits of segmenting entrepreneurs in business support program according to their leverage of targeted feedback mechanisms.

\subsection{INTRODUCTION}

Feedback plays an important role in entrepreneurship (Markman et al., 2002; Ozgen \& Baron, 2007). Targeted feedback typically comes from coaches and mentors (St-Jean \& Audet, 2012) but can also come from relevant stakeholders, such as experts or colleagues in the industry (Ozgen \& Baron, 2007) as well as investors (Collewaert \& Sapienza, 2014) or panel presentations (Miron-Schatz et al., 2014; Gerasymenko et al., 2015).

Targeted feedback helps entrepreneurs to develop their business opportunity successfully (Shepherd \& Krueger; 2002; Rice, 2002) because it results in important learning effects of entrepreneurs (Ozgen \& Baron, 2007). It has a positive effect on opportunity identification (De Carolis \& Saparito, 2006; Ozgen \& Baron, 2007), increases self-efficacy (Markman et al., 2002; St-Jean \& Aubret, 2012), enhances customer understanding and technical learning (Rice, 2002) and facilitates entrepreneurial (cognitive) learning (St-Jean \& Audet, 2012).

In particular, the literature has recognized the influence of targeted feedback on entrepreneurial cognitive development (St-Jean \& Audet, 2012). Studies suggest that cognitive development is crucial in moving from a novice entrepreneurial mindset to a more 'expert' entrepreneurial mindset (Dreyfus \& Dreyfus, 2005; Krueger \& Day 2010), underlining the importance of cognitive aspects of opportunity development. 
While existing studies on the effect of coaching on entrepreneurs have provided valuable insights, they have typically treated entrepreneurs as a homogeneous group. Studies on this topic have focused on mentoring or coaching effects on novice entrepreneurs (Allen et al, 2005; Kutzhanova et al, 2009; St-Jean \& Audet, 2012), at the detriment of examining the effect of targeted feedback on different categories of entrepreneurs. But as research suggests, entrepreneurs' age and education affect whether entrepreneurs benefit from targeted feedback provided by mentors (Bisk, 2002). Also, we lack insights into the effects of different forms of targeted feedback on the cognitive development of entrepreneurs.

In this study, we undertake a systematic comparison of the ways in which two forms of targeted feedback - coaching and panel presentation feedback - affect entrepreneurial development during opportunity development. Using a grounded method approach we examined longitudinal, real-time logbook data of 70 entrepreneurs inside a business incubation/acceleration program.

We develop a taxonomy based on the effects of different forms of targeted feedback on entrepreneurial cognitive development. We find that coaching and panel feedback have different effects on entrepreneurial cognitive learning. In particular, the way targeted feedback mechanisms influence the entrepreneur's cognitive development is affected by the type of value offer developed - product or service - as well as by prior entrepreneurial experience.

Our findings yield four distinct categories of entrepreneurs which we labeled, respectively, greenhorns, cowboys, trappers and saloon-owners. With this study we contribute to empirical evidence of the development of entrepreneurial metacognition, and more generally how feedback mechanisms affect entrepreneurial cognitive development. More practically, we provide insights into the ways in which institutional business support can optimally stimulate entrepreneurial cognitive development during opportunity development. 


\subsection{THEORY}

\section{TARGETED FEEDBACK AND ENTREPRENEURSHIP}

In the literature, targeted feedback is found to affect entrepreneurial development and performance. For example, Ozgen \& Baron (2007) in a study of the effects of mentors, industry networks and professional forums on opportunity recognition found that all three feedback sources have direct, positive effects on novice entrepreneurs' opportunity recognition. And Haynie et al.'s (2010b) study demonstrates that there is positive relationship between targeted (cognitive) feedback and entrepreneurial performance. The authors distinguish between outcome-based - defined as feedback associated with performance-oriented information relative to an objective standard - and cognitive feedback which is described as information about the relations in the environment, relations perceived by the person and the relations between the environment and the person's perception. The results show that that, given a dynamic decision task in a business context, cognitive feedback promotes significant normative improvements in decision accuracy. These studies underline the positive effects of targeted feedback on entrepreneurial cognitive development.

Prior research also found that different feedback sources influence the way in which individuals are affected by feedback. For example, individuals are affected differently by feedback depending on the credibility of the feedback source (Son \& Kim, 2014) and they prefer advice of some parties to that of others (Fischer \& Reuber, 2003). And Bisk (2002) proposes that entrepreneurs' age and education are factors that affect the benefits which entrepreneurs perceive to obtain from targeted feedback in the form of mentoring. These studies indicate that the source of feedback matters, and that personal attributes influence the way in which entrepreneurs are affected by targeted feedback. Although the variability of feedback effect coming from different sources has been established (Son \& Kim, 2014), this variability has not yet been examined among entrepreneurs.

\section{FORMS OF TARGETED FEEDBACK}

It has been recognized that interventions at pre-start and start-up stages of a business help to reduce high failure rates (Deakins et al., 1998). In that way they contribute to successful business incubation. It is therefore not surprising that targeted feedback processes are prominent dimensions of the entrepreneurial ecosystem 
(Gulbranson \& Audretsch, 2008; Cohen \& Feld 2011). Prominent examples of targeted feedback for entrepreneurs are coaching and mentoring. These aim at skill development and performance enhancement, in addition mentoring is geared towards longer term career development (Passmore, 2007). In addition, pitch or panel presentations, for example for investors (Pollak et al, 2012), offer targeted feedback opportunities.

This study focuses on two forms of targeted feedback: coaching/mentoring feedback and feedback received in panel presentations.

\section{COACHING AND MENTORING}

An increasingly popular intervention during venture start-up is that of targeted feedback via coaching or mentoring. While there are differences between coaching and mentoring, they resemble each other in the sense that they both are geared towards the mentees/coached individual's career/professional and personal development - albeit in (slightly) different ways relating to process and content (Crompton \& Smyrnios, 2006; Crompton et al., 2012).

There is an upsurge of business incubation and acceleration programs with an emphasis on coaching, mentoring or other counselling services (Rice, 2002; Scillitoe \& Chakrabati, 2010). Authors have pointed to intensive mentoring processes which have become key to accelerator programs (such as TechStars.org; see Gulbranson \& Audretsch, 2008).

Studies have found that targeted feedback from mentors increases the mentees self-efficacy (Saadoui \& Affess, 2015), his or her self-confidence as well as self-esteem (Waters et al. 2002). Targeted feedback has also been found to augment the mentee's ability to achieve goals, identify problems, learn, manage the firm and deal with change (Deakins et al., 1998) and to increase his or her knowledge and contact network (Wikholm et al., 2005).

Radu et al. (2013) show that mentors influence entrepreneurs' attitudes and behaviour by means of a number of communicational strategies: persuasion, engagement, criticism, and provocation, while the impact of these strategies are categorized in terms of commitment, compliance or resistance. St-Jean \& Audet (2014) studied the intervention style used by mentors with entrepreneurs, and found that low directivity (the maieutic style) together with a high level of mentor involvement in the mentoring relationship generates the most positive outcomes for the mentees, whereas 
high directivity in combination with low level of involvement of the mentor leads to poorer results for the mentee.

Previously, St-Jean \& Audet (2012) also examined the effects of coaching on skillbased, affective and cognitive learning of entrepreneurs. They found that cognitive learning plays the biggest role in coaching and distinguish between three different forms of cognitive learning outcomes by novice entrepreneurs: verbal knowledge, knowledge organization and cognitive strategies.

\section{Panel presentations}

In addition to targeted feedback in the form of coaching and mentoring, targeted feedback is increasingly studied in a 'pitch' or panel presentation situations. This form of targeted feedback consists of a rather short verbal presentation of the business idea to a panel of (relevant) experts or other (potential) stakeholders, such as five-minute verbal pitches that are used by venture capital firms and entrepreneurship competitions. Panel feedback has gained publicity and popularity by being used on television shows such as Mark Burnett's Shark Tank or Sony Pictures' Dragon's Den. In these shows, aspiring entrepreneurs present their business idea to a panel of very wealthy private investors.

Studies have examined the preparedness of entrepreneurs when giving presentations to venture capitalists (Chen et al., 2009) and looked at the impact of the entrepreneurs' presentation skills and preparedness on business angels' initial investment decisions (Clark, 2008; Galbraith et al., 2013).

Pollack et al. (2012) describe how entrepreneurs use the pitch presentation to enact their business opportunity and enlist the help of stakeholders. Entrepreneurs do so by guiding financiers to make sense of the venture as if it already existed, through the use of a narrative. The authors argue that new venture entrepreneurs can possibly better focus on achieving legitimacy, not necessarily on directly acquiring financial resources, because resources are granted after legitimacy is achieved.

According to Miron-Schatz et al. (2014) panel presentations have gained increasing importance in business opportunity development (and even perpetrated the context of institutionalized, regulated settings. The authors describe conferences which organize panel presentations, such as the Health Information Management Systems Society (HIMSS) conference and how entrepreneurial education program increasingly incorporate panel presentations in their business incubation/acceleration curriculum. 


\section{TARGETED FEEDBACK AND COGNITIVE DEVELOPMENT}

Cognitive development is associated with the development of knowledge structures which help us organize the way we interpret, analyze, remember, and use information about the social world (Mitchell et al., 2007). Entrepreneurial cognition plays an important role during opportunity identification and preparation (Narayan et al., 2011; Kor et al., 2007; Baron \& Ensley, 2006). Studies found various positive effects of targeted feedback on entrepreneurial cognitive development. For example, targeted feedback from various sources can help in identifying business opportunities (Ozgen \& Baron, 2007).

Targeted feedback is a critical ingredient in evolving the entrepreneur's cognition alongside the entrepreneur's venture (Krueger, 2007; 2009). In this study we focus on the effect of targeted feedback on entrepreneurial cognitive development in terms of cognitive learning outcomes.

\section{Cognitive LEARNING OUTCOMES}

Three forms of cognitive learning outcomes are distinguished in the literature (Kraiger et al., 1993). These are 1) declarative knowledge which is associated with verbal knowledge of concrete pieces of information, for example a name, a concept, a theory or even how to produce statements - usually related to a specific area of expertise; 2) knowledge organization, or procedural knowledge, for example about different stakeholders' perspectives and 'linking knowledge together'; and 3) cognitive strategies which are associated with the ability to find the best problem-solving strategy for a particular problem.

Kraiger et al. (1993) proposed that only cognitive strategies are associated with metacognitive abilities because cognitive strategies are related to learning about one's goals or 'learning to learn': "The term metacognition has been used to refer to both the knowledge of one's own cognition and the regulation of such (Brown, 1975; Leonesio \& Nelso, 1990). Metacognitive skills include panning, monitoring, and revising goalappropriate behavior (Brown et al, 1983; Schoenfeld, 1985) or understanding the relationship between task demands and one's capabilities (Pressley et al, 1987). They also include skills in regulating or evoking appropriate strategies (Beretier \& Scardamalia, 1985). Strategies refer to a broad range of mental activities that facilitate knowledge acquisition and application (Prawat, 1989)." (Kraiger et al., 1993) 
The difference between cognitive skills in the form of verbal/declarative knowledge, knowledge organization, and cognitive strategies has also been empirically and successfully examined in the context of entrepreneurship by St-Jean \& Audet (2012). The authors found a positive correlation between targeted feedback from mentors and the development of entrepreneurial cognition in terms of these cognitive learning outcomes. Their results show that novice entrepreneurs report cognitive development the most in terms of knowledge organization, closely followed by the development of verbal knowledge and to a lesser extent that of cognitive strategies.

The findings of St-Jean \& Audet (2012) about different cognitive learning outcomes as a result of coaching offer valuable insights into the ways in which entrepreneurial cognition is affected by targeted feedback. In this study we conceptualize the effects of targeted feedback in terms of these different learning outcomes. That is elaborated in more detail in the method section.

\section{TAXONOMY DEVELOPMENT BASED ON THE EFFECTS OF TARGETED FEEDBACK MECHANISMS}

Existing researches focuses on the process and impact of targeted feedback by means of mentoring among nascent entrepreneurs. However, Bisk (2002) studied the satisfaction of entrepreneurs with the mentoring relationship and their perception of the effectiveness of their assigned mentors and found that entrepreneurs' age and education are factors that impact on whether they benefited from the mentoring engagement. Since we are in particular interested in analyzing the effects of targeted feedback mechanisms on entrepreneurs with varying amount of prior entrepreneurial experience, we set out to develop a taxonomy of feedback effects.

\section{TAXONOMY OF ENTREPRENEURS}

Both taxonomy and typology development target the identification and conceptualization of distinctive categories of individuals, on the basis of characteristic (dis)similarities of individuals, groups or other (social) entities. A glance upon the literature in entrepreneurship shows that studies have mainly focused on entrepreneurial typologies.

Typologies in entrepreneurship are for example based on differences in motivations, expectations and goals which entrepreneurs have (Filley \& Aldag, 1978; Cooper \& Dunkelberg, 1986; Lafuente \& Salas, 1989). Other typologies are based on the degree of entrepreneurs' resource possession and ways of resource acquisition (Jarillo, 1989), their venture set-up strategy (Filion, 2004) or the composition of founding 
teams and founder's background characteristics such as education or experience (Smith \& Miner, 1983; Lorraine \& Dussault, 1987). While these studies show the relevance of categorizing entrepreneurs according to particular attitudes, behaviors and strategies, they leverage existing variables and concepts in order to depict their categorization results.

Contrary to a typology, a taxonomy is inductively developed. A taxonomy offers a framework for the identification and conceptualization of distinctive categories of individuals, on the basis of characteristic (dis)similarities of individuals, groups or other (social) entities. For example Fauchart \& Gruber (2011) found that the social identity of a firm founder not only determines how he or she accords meaning to venture development, but also influences firm growth. This shows that induction-based theory development is relevant for a better understanding of venture development processes and ultimately performance.

The relevance of taxonomy development is the reason why we decided to develop a taxonomy in this study. Since there are no systematic conceptualization of how different targeted feedback mechanisms affect entrepreneurial cognitive learning outcomes differently, we aim to establish such a taxonomy based on empirical, qualitative data.

\section{SUMMING UP THE THEORETICAL APPROACH}

In this study we seek to identify the effects of different forms of targeted feedback among entrepreneurs of varying age and experience and from various sectors. In order to analyze those effects in such a varied sample and contribute to theory-building on this topic, we set out to develop a taxonomy of the effects of coaching and panel feedback on cognitive learning outcomes previously used by St-Jean \& Audet (2012). To carry out this empirical analysis, we employ a grounded theory approach that we present next.

\subsection{METHOD}

\section{DESIGN}

Since there are no systematic conceptualization of how different targeted feedback mechanisms affect entrepreneurial cognitive learning differently, we aim to establish a taxonomy based on empirical, qualitative data. We employ a qualitative research method 
which provides us with the opportunity to inductively identify and compare the effects of feedback mechanisms on entrepreneurs, as well as develop a taxonomy of entrepreneurial feedback leverage.

\section{GROUNDED THEORY}

In this study, we use the grounded theory procedure for the analysis of the effect of feedback mechanisms. Grounded theory is a way of thinking about and conceptualizing of data, and its ontological roots can be found in the importance of discovery (Corbin \& Strauss, 1990; Strauss \& Corbin 1994). It is a qualitative research method based on the careful and systematic collection of data and its analysis, using the cross-comparison method in the analysis of empirical data, with the aim of establishing conceptual relationships (Strauss \& Corbin, 1994).

The grounded theory method is characterized by systematic coding procedures, and constantly making comparisons while analyzing the data. In grounded theory, coding is seen as an important element of transforming raw data into theoretical constructions of social processes (Kendall, 1999). This leads to concept development with 'considerable meaningful variation' which is the base for theory development (Strauss \& Corbin, 1994).

Because grounded theory involves the development of theory, based on inductive (empirically-based) research, it contributes to theory generation (Strauss \& Corbin, 1994) or theory elaboration. Theory elaboration develops or refines theories further by specifying more carefully "the circumstances in which it does or does not offer potential for explanation" (Vaughan, 1992: p. 175). Grounded theory, based on empirically-derived categories and their distinctiveness, is therefore very well suited for the development of a taxonomy.

Corbin \& Strauss (1990) distinguish three different coding processes in grounded theory; open coding, selective coding and axial coding. Open coding is a coding process which involves the breaking down data analytically to arrive at new way of thinking about or interpreting phenomena reflected in the data. In selective coding, all categories are unified around a central 'core' category and are filled-in with descriptive detail. Axial coding is applied when the 'coding paradigm' is used for relating (possibly theory-based) categories and subcategories, and to then test their relationships against data.

Axial coding combines the advantages of open and selective coding, by being 'more concertedly' than open coding, according to Corbin \& Strauss (1990), yet more 
open than selective coding because axial coding leaves room for more than one core category. It is a 'coding paradigm' which orders data and the relationship between concepts in terms of the following aspects: phenomena, conditions, (inter)actions and consequences (Corbin \& Strauss, 1990). This coding process provides reference points yet offers enough flexibility to inductively construct core categories.

Because of aforementioned advantages this study we use axial coding to examine the phenomenon of feedback in its conditions, (inter)actions and consequences pertaining to coaching and panel feedback. In the case of our study the central phenomena are two forms of targeted feedback, coaching and panel feedback. The conditions are examined in terms of background characteristics of entrepreneurs as well as their business opportunity. The (inter)actions involved are those of feedback interactions, focusing on instances of both forms of targeted feedback, coaching and panel feedback, during business opportunity development.

As mentioned earlier, we analyze the consequences of coaching and panel (inter)actions in terms of three cognitive learning outcomes identified in the literature (Kraiger et al., 1993) and which have been successfully applied to the study of coaching feedback outcomes (St-Jean \& Audet (2012), namely declarative knowledge, procedural knowledge and cognitive strategies. Below the sampling and coding procedure are described in more detail.

\section{SAMPLING AND SAMPLE CHARACTERISTICS}

We applied purposeful sampling in this study in order to attain a varied sample of respondents. It was drawn from the population of entrepreneurs who participated in the one-year business incubation/acceleration program at the Venture Lab Twente (VLT).

The VLT program consists of a number of support structures, among which weekly coaching and three-monthly panel presentations. Coaching as well as panel presentations represent instances of targeted feedback which are offered equally to all participants throughout the incubation/acceleration program. The regular frequency of both targeted feedback mechanisms make the incubation/acceleration program a setting which is characterized by a controlled taking place of targeted feedback events. This condition satisfies our requirement of studying both targeted feedback mechanisms and thus makes the incubation/acceleration an attractive setting for our study.

Respondents were selected from the population of entrepreneurs who had finished the one-year business acceleration program and exited this program in the recent past. 
These entrepreneurs were still involved in business opportunity development and/or had already started to commercially exploit it at the time of exiting the VLT program.

Our initial sample contained 70 respondents of which we discarded those respondents who were either not self-employed (for example they had a franchise or were family entrepreneurs and not starting a novel business opportunity) or they had stopped developing their business opportunity.

The final sample consisted of 54 entrepreneurs who had finished the incubation/acceleration program at the time of data collection for this study. The distribution of gender in our final sample was extremely unequal due to a high participation of male entrepreneurs in the business accelerator program; there are only 5 female entrepreneurs in our sample.

The respondents in our sample vary in their amount of prior entrepreneurial experience, age group, educational and employment histories as well as business opportunity. The average age of entrepreneurs in our sample is 45 years, with an average of 15 years of work experience and 7 years of entrepreneurial experience.

Regarding the educational background of respondents, it must be noted that the sample is not entirely evenly distributed across the various educational fields: 34 entrepreneurs have an education in the fields of natural sciences and engineering (which we label 'technical' education) as opposed to 22 entrepreneurs with an education in the social sciences and humanities (which we label 'non-technical' studies). This means that our sample contains more respondents with a 'technical' than with a 'non-technical' education, probably due to the incubation/acceleration program's physical and organizational proximity to a technical university and a science park as well as its profile as a high tech start-up incubuator/accelerator.

\section{MEASURING INSTRUMENT}

The data used in this study is derived from the so-called 'diaries', in the form of weekly, digitally submitted self-reports entries into an online logbook-keeping system set up for the Venture Lab Twente.

To measure the effect of the two forms of feedback in this study we use data from digital diaries. These digital diaries are part of a larger university-based research program among entrepreneurs of the incubation/acceleration program at the Venture Lab Twente. Keeping of the diary was a requirement for the entrepreneurs to take part 
in the program. We only selected entrepreneurs in our sample who had written in their logbook 20 times or more throughout the program.

Diary studies have a long tradition in the field of psychology because they offer the opportunity to investigate micro-level processes within their natural context. Diary data is suitable for the development of a taxonomy because diary data provides reliable person-level information (Bolger et al., 2003) and can be used to compare individual differences in that kind of information. Since we compare the effects of feedback mechanisms on the micro-level a diary analysis fits very well with the purpose of our study. A particular strength of diary research is the high validity and reliability of the qualitative data collected through nearly real-time accounts of individual experience with reduction in biases due to retrospection.

The psychology literature distinguishes between three types of diary collection methods; interval, signal, and event contingent diary design. For this study the intervalcontingent protocol (Wheeler and Reis, 1991; Bolger et al., 2003) is used. The intervalcontingent protocol requires participants to communicate their experiences at regular, predetermined intervals. Because of the ongoing, dynamic and uncertain nature of entrepreneurial action, entrepreneurs may not be reflexively aware of which events or signals deserve reporting.

By establishing frequent and regular moments of reporting, we aimed to minimize the loss of data of skewed reports due to 'retrofitting'. Retrofitting refers to the facts that the longer an event is in the past, the higher the chance that a person is prone to forget (relevant) details or succumb to nostalgia by sketching a situation or event as more advantageous, beneficial or positive that it might have actually been.

Furthermore, the use of regular intervals allowed us to make the habit of filling out the diaries an integral part of the program. In the case of this study, the intervals of the diaries were weekly. Weekly intervals were judged as appropriate since they allow sufficient time for entrepreneurs to deploy their regular activities while being able to report on the progress of their ventures with as little retrospection as possible.

The diaries address four topics explicitly; these are 'learnings', 'results', 'issues' and 'next steps'. The diaries are filled in and kept digitally; entrepreneurs were able to access and write their weekly diaries digitally and university-based researchers monitored and stored the data and made them accessible to us. Anonymity for the program participants and discretion in handling the data were guaranteed to all 
participants and strictly respected by the researchers throughout the process of data collection and analysis.

On average, respondents wrote diaries 37 times during the program, which translates into an average of 37 weeks of diary data per person. Considering the incubation/acceleration program runs one year - about 52 weeks -, on average respondents provided diary entries at more than two thirds of their time of the program. Therefore the amount of diary entries provides a reliable source of information about the entrepreneurs' perception and interpretation of their time in the program.

\section{DATA CODING}

The unit of analysis is a report of effects of phenomenon coaching/mentoring and panel feedback, as well as its conditions and interactions, in the diary text. In total we coded about 10,500 diary entries. On the bases of Nvivo rapports of search engine outcomes, we identified a total of 402 instances in the diaries which related to the two mechanisms of targeted feedback. We then applied grounded theory in order to identify and compare leverage by entrepreneurs of the two targeted feedback mechanisms of coaching and panel presentations. To do so, we employed first- and second-order coding steps. These are explained below.

\section{FIRST-ORDER CODING: THEMATIC CATEGORIES}

The data collected from the digital diaries was first entered into a dataset in the software program QSR NVivo 10.0. NVivo is an inductive, systematic and flexible coding tool in which conceptual categories can easily be coded digitally. We coded the empirical data in the form of self-reports about (inter)actions and their consequences related to coaching and panel presentations into thematic categories.

To code into thematic categories, we look at how entrepreneurs make sense of the feedback interactions reported in their diary entries. Sensemaking processes are driven by the individual actor's seeking of plausibility of events and situations (Weick, 1995; Humphreys et al., 2012) and they are interpretative processes by which actors pick up cues ('signaling'), note them ('bracketing') and categorize them ('labeling') (Weick, 1995; Weick et al., 2005). The grounded theory approach can fruitfully be combined with the sensemaking perspective because the latter allows to examine changes in actors' cognitive structures, in particular when studied temporally (Strike \& Rerup, 2016), as is the case in this research. 
Two coders separately coded the instances in the diaries in which feedback interactions and results were mentioned (as explicitly noted by the entrepreneurs themselves). The two coders then compared the identified sensemaking reports of respondents about coaching or panel (inter)actions and their results against other reports and discussed similarities and differences. On the basis of these comparisons and discussions, we construed so-called content-oriented thematic data points (Seale, 1998). By means of cross-comparison of the data and inter-rater comparison of coding results we identified a number of recurring sensemaking 'themes' that could be associated with a more encompassing thematic categories. These thematic categories range from an increased awareness for business planning and modelling, to a more positive attitude towards potential customers and an increased awareness of the importance of stakeholders. The former, then, was assigned the thematic label 'business plan/model', whereas the latter two are grouped together under the thematic label 'market orientation'.

On the basis of the thematic categories and the reported amount of (inter)actions with coaches respectively panel members we identified similarities and differences between entrepreneurs in our sample. In addition, the coders compared the background of the respondents for similarities and differences. For example, we noticed that some entrepreneurs reported more interaction with panel members than others, and that some entrepreneurs reported some learning thematic learning outcomes more than others. Entrepreneurs reporting similar patterns of (inter)actions with coaches respectively panel members and similar thematic learning outcomes were then grouped together.

\section{SECOND-ORDER CODING: COGNITIVE LEARNING OUTCOMES}

The second-order coding of the data contains the coding of thematic learning outcomes into declarative knowledge (verbal learning), procedural knowledge (knowledge organization) and learning of cognitive strategies (including metacognition) described by St-Jean \& Audet (2012), or a combination of those three categories where applicable and agreed upon by both coders.

Data was only coded if specific signal words would allow the coders to do so. This means that for example the sole mention of received feedback or report of discussions with a coach, without any further content-related sensemaking by the respondents, would not be coded.

A learning outcome is coded as declarative (verbal) knowledge when the diary report mentions general information, referrals to people, networks or other suggestions 
such as the request of a coach for a better or different definition of the venture's goals, mission or vision. For example, a respondent wrote in his diary: "My coach has given me the address of [a contact person working at the Chamber of Commerce]; this is about patent advice." This quote contains the reference to a concrete piece of information that the entrepreneur received from his coach - the name of a contact person who can help with the development of the opportunity in the form of patent advice - and is therefore coded as reflecting the development of declarative knowledge.

A learning outcome is coded as procedural knowledge (knowledge organization) when the diary entry relates to feedback regarding how the entrepreneur had obtained information or knowledge about how to engage in certain venture-related activities such as the strategy, focus areas or the marketing plan. An example of procedural knowledge development is shown in the following quote: "Last Tuesday I had my first (official) meeting with my coach. So far, [the company] gets its assignments by having good relationship with technicians at customer side. A new approach might be to attack the management with a solution orientated presentation." We see here that the coaching interaction has led to the consideration of how to acquire new assignments - namely, by means of a 'solution oriented presentation' -which reflects the development of procedural knowledge.

Learning outcomes related to the entrepreneurs' own role in their venture (for example, discussions about the personal development plan of the entrepreneur) but also the venture's business model or business plan are coded as both declarative and procedural knowledge. This is due to the fact that it is not possible to distinguish between content and process of in thematic outcomes - they are characteristically intertwined.

A learning outcome in which entrepreneurs reflect on and/or abstract their learning from the concrete situation or event at hand to a more general learning outcome are coded into cognitive strategies (metacognitive development). For example, an entrepreneur described the outcome of coaching interaction in the following way: "Most important session was with personal coach. In talking to her I came to understand my drivers and obstructions better. In particular the awareness what my character is capable of doing and not doing makes me more determined in the way forward: my energy and ability to stimulate people has great potential but also a danger of over-asking. I need to build in personal reflection time and rest moments to consider those risks and dangers." This quote contains a reflective learning instance - the building-in of personal reflection 
time to consider risks and dangers associated with opportunity development - which demonstrates the development of a cognitive strategy and is therefore coded as such.

Throughout both first-order and second-order coding and the analysis, the coders continuously cross-compared the various diary reports and sought to categorize respondents who show similarities. This regards not only similarities in the consequences reported in the diaries (in terms of thematic and cognitive learning outcomes of coaching and panel feedback) but also similarities in the respondents' background and the amount of (inter)actions they reported.

This cross-comparison yielded four distinctive categories of entrepreneurs which are described in the results section.

\subsection{RESULTS}

The results of our analysis are presented below. We first give a short general overview of the main findings, before elaborating them in more detail.

\section{GeNERAL FINDINGS}

Overall we noticed that targeted feedback from coaches is reported almost twice as frequently as feedback from the panel presentation. On average, respondents mention coaching 7.1 times and panel feedback 3.7 times in their diaries. This difference in amount of instances reported is most likely related to the more frequent amount of coaching interaction instances as opposed to panel interaction instances; coaching takes place once a week whereas panel presentations take place every three months.

We also noted that not all references to coaching or panel feedback interaction in the diaries contain actual information about the effect of that interaction: A little more than a fifth $(22 \%)$ of all coaching or panel interaction is reported in the dairies, simply contain a mention of the respondents that they should be or are preparing and/or planning coach meetings respectively panel presentations. Those are discarded from the analysis.

We noticed that differences between the four types of entrepreneurs mainly stem from two conditions: the nature of the business opportunity - whether the business 
opportunity was service-based or product-based - and the prior entrepreneurial experience of the respondents. The results of our analysis yield four distinctive categories of entrepreneurs with regards to the cognitive effects associated with targeted feedback (inter)actions. Entrepreneurs of each category convey different cognitive learning outcomes with regards to the two feedback mechanisms. Based on the analysis we categorized entrepreneurs in our sample in one of four categories. Each of these categories conveys resembling characteristics with a typical personage found in classic American 'Wild West'-movies and were named, accordingly, 'Greenhorns', 'Cowboys', 'Trappers', 'Saloon-owners'. The different categories are described below.

\section{GREENHORNS}

Entrepreneurs in this category are novice to business opportunity development and characteristically do not have a delineated business plan yet. They seek to develop a service-based business opportunity, however more than entrepreneurs in the other categories they lack customer focus, self-confidence and a pro-active 'go-getter' mentality. Entrepreneurs in this category are labeled as 'Greenhorns', a name for a person new to the 'Wild West' of America and vulnerable to its dangers. While they are ambitious and willing to enter a world of uncertainty - that of business opportunity development - they lack the resources, networking skills or knowledge about where to get the right resources needed - whether it be information, finances, or potential customers.

\section{CowBoys}

Entrepreneurs in this category develop a product-offer and have a strong technology background (most commonly in computer or natural sciences and engineering). While they lack prior entrepreneurial experience, they are able to quickly develop the business opportunity; this category is characterized by a pro-active and very goal-oriented attitude. Entrepreneurs in this category can be described as 'Cowboys' who are without fear of the uncertainty of business opportunity development. They are very committed to and focused on developing their business opportunity. They leverage their (technological) skills in order to enter unknown markets - just as cowboys in the Wild West leveraged the benefits of a fast horse to ride deep into unknown territories.

\section{TRAPPERS}

Entrepreneurs in this category have prior experience with the development of a business opportunity, however that opportunity is service-based; now they seek to move 
from a service-based offer to a product-based offer. While they are seasoned veterans in entrepreneurial activities, they are new to the dangers and benefits of value creation in terms of a product-offer; for example, they lack specific knowledge of the nature of product development, such as technology and human resource management involved in scaling the business towards product development. Entrepreneurs in this category can be described as 'Trappers', those individuals in the Wild West who travelled alone in unknown, unchartered territory. They often seek encouragement and sparring partner in feedback instances, while also being critical and goal-oriented; in that sense, this category combines the characteristic self-confidence issues of greenhorns with the goaloriented focus of the cowboys.

\section{SALOON-OWNERS}

Entrepreneurs in this category have as much entrepreneurial experience as the trappers, however they had started with a product-based business opportunity and have seen growth of the venture throughout the past. They employ staff and are used to the creation, capture and delivery of a product-based value offer. Entrepreneurs in this category are in a comfortable position reminiscent of that of saloon-owners in the Wild West who owned a resource-rich establishment, with a steady income and a customerbase in a relatively safe environment. They are therefore labelled 'Saloon-owners'. These entrepreneurs seek to differentiate their offer or even develop a completely new product, and are therefore faced with the challenges of novel business opportunity development.

Table 2-1 depicts the main characteristics of each of the four categories of entrepreneurs in terms of their background, as well as interactions with and consequences of coaching and panel. Those consequences of coaching and panel feedback are grouped in thematic categories identified in the entrepreneurs' diary reports.

Table 2-1: Themes and their frequency among the different types of entrepreneurs

\begin{tabular}{|c|c|c|c|c|}
\hline \multirow[t]{2}{*}{ Theme } & \multicolumn{4}{|c|}{$\begin{array}{l}\text { Categories of entrepreneurs (leverage of coaching } \\
\text { feedback, \% of total reports per category) }\end{array}$} \\
\hline & Cowboys & $\begin{array}{c}\text { Greenhorn } \\
\text { S }\end{array}$ & Trappers & Saloon-owners \\
\hline Identity & $14 \%$ & $12 \%$ & $9 \%$ & $15 \%$ \\
\hline Business model/plan & $7 \%$ & $22 \%$ & $17 \%$ & $14 \%$ \\
\hline Market orientation & $34 \%$ & $8 \%$ & $5 \%$ & $7 \%$ \\
\hline
\end{tabular}




\begin{tabular}{|c|c|c|c|c|}
\hline Communication & $2 \%$ & $3 \%$ & $8 \%$ & $0 \%$ \\
\hline Time management & $7 \%$ & $3 \%$ & $3 \%$ & $2 \%$ \\
\hline Strategy & $5 \%$ & $8 \%$ & $4 \%$ & $8 \%$ \\
\hline Team & $7 \%$ & $2 \%$ & $2 \%$ & $7 \%$ \\
\hline Network & $5 \%$ & $2 \%$ & $5 \%$ & $2 \%$ \\
\hline Focus & $2 \%$ & $7 \%$ & $3 \%$ & $0 \%$ \\
\hline Presentation & $0 \%$ & $2 \%$ & $4 \%$ & $8 \%$ \\
\hline Reflection & $0 \%$ & $5 \%$ & $5 \%$ & $0 \%$ \\
\hline General/diverse & $0 \%$ & $5 \%$ & $8 \%$ & $0 \%$ \\
\hline feedback/tips & $0 \%$ & $0 \%$ & $1 \%$ & $0 \%$ \\
\hline Finances & $0 \%$ & $2 \%$ & $0 \%$ & $0 \%$ \\
\hline Subsidy & $0 \%$ & $1 \%$ & $0 \%$ & $8 \%$ \\
\hline Intellectual property & & & & \\
\hline
\end{tabular}

The thematic categories cover a wide range of topics. One of the dominant outcomes of targeted feedback is the entrepreneur's identity. Another important theme relates to the development of the venture's business model or plan and the entrepreneur's market orientation. Other frequently mentioned targeted feedback pertain to issues of communication, time management, as well as the entrepreneur's team or network development, and the development of this strategy. Other thematic learning outcomes we identified relate to learning to focus, and the importance of presentations and reflection. Thematic categories related to more material themes - such as finances, subsidy and intellectual property (IP) were sparsely identified.

In graph 2-1 and graph 2-2 we show the cognitive learning outcomes which result from coaching respectively panel feedback interaction. Right below that in table 2-2 we give an overview on the main characteristics of the four different categories of entrepreneurs, as well as the different cognitive learning outcomes. Those four categories are then presented in more detail. 
Graph 2-1 Cognitive learning outcomes as a result of coaching feedback

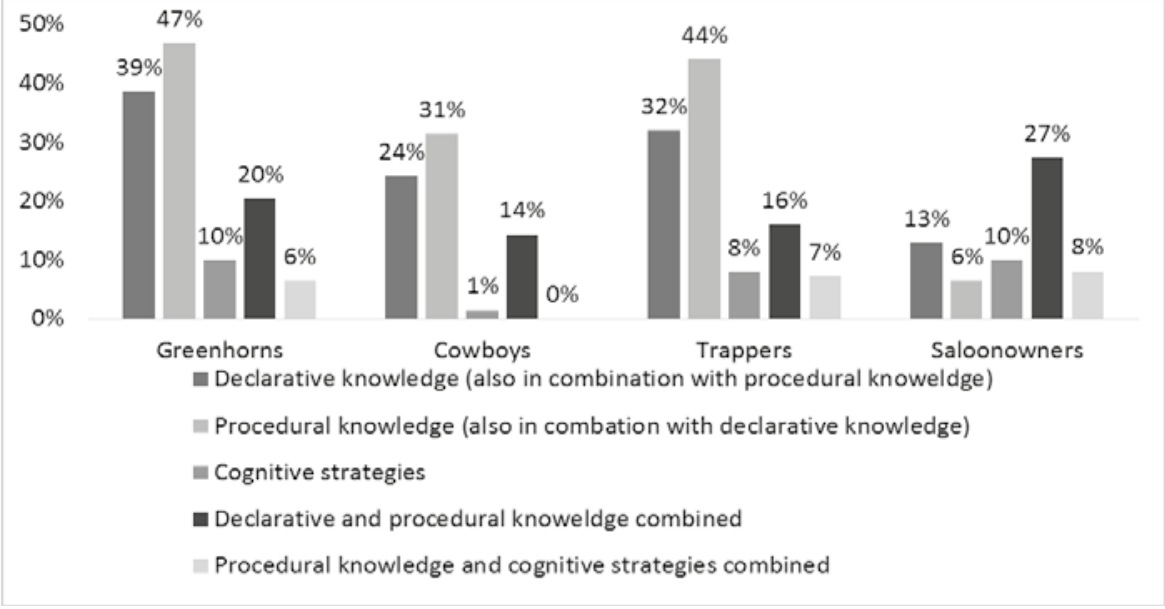

Graph 2-2 Cognitive learning outcomes as a result of panel feedback

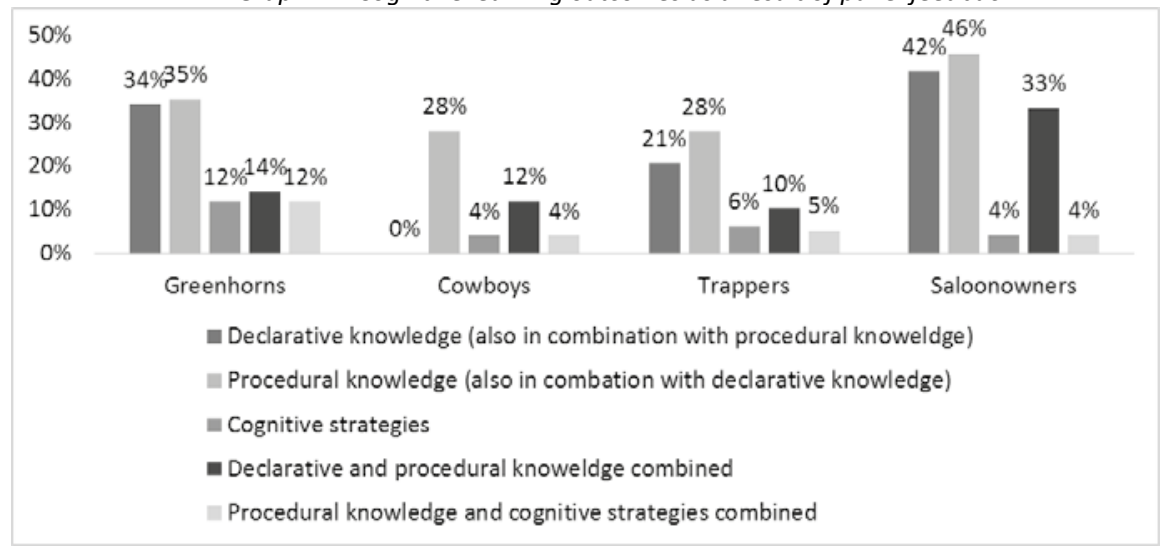




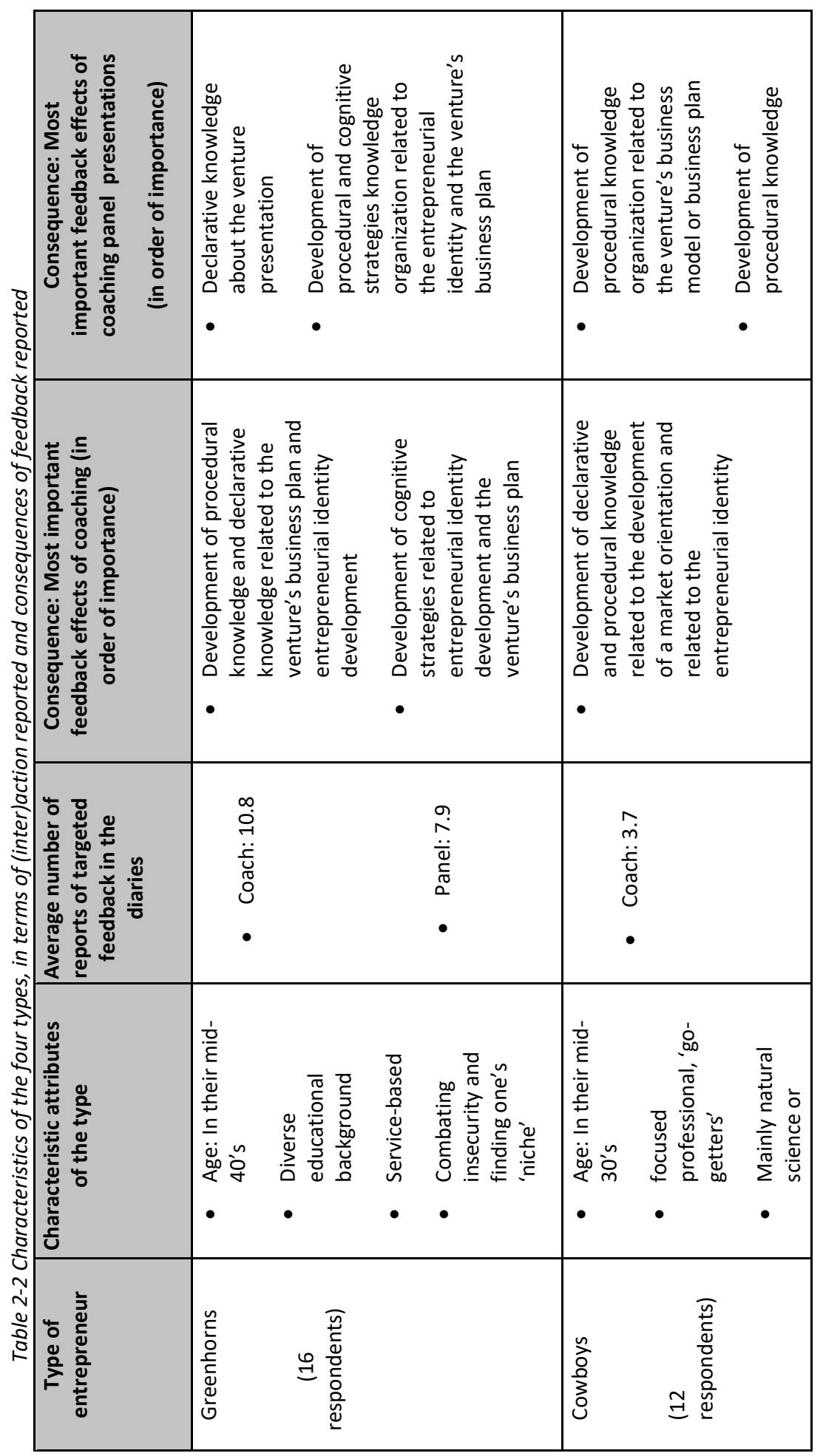




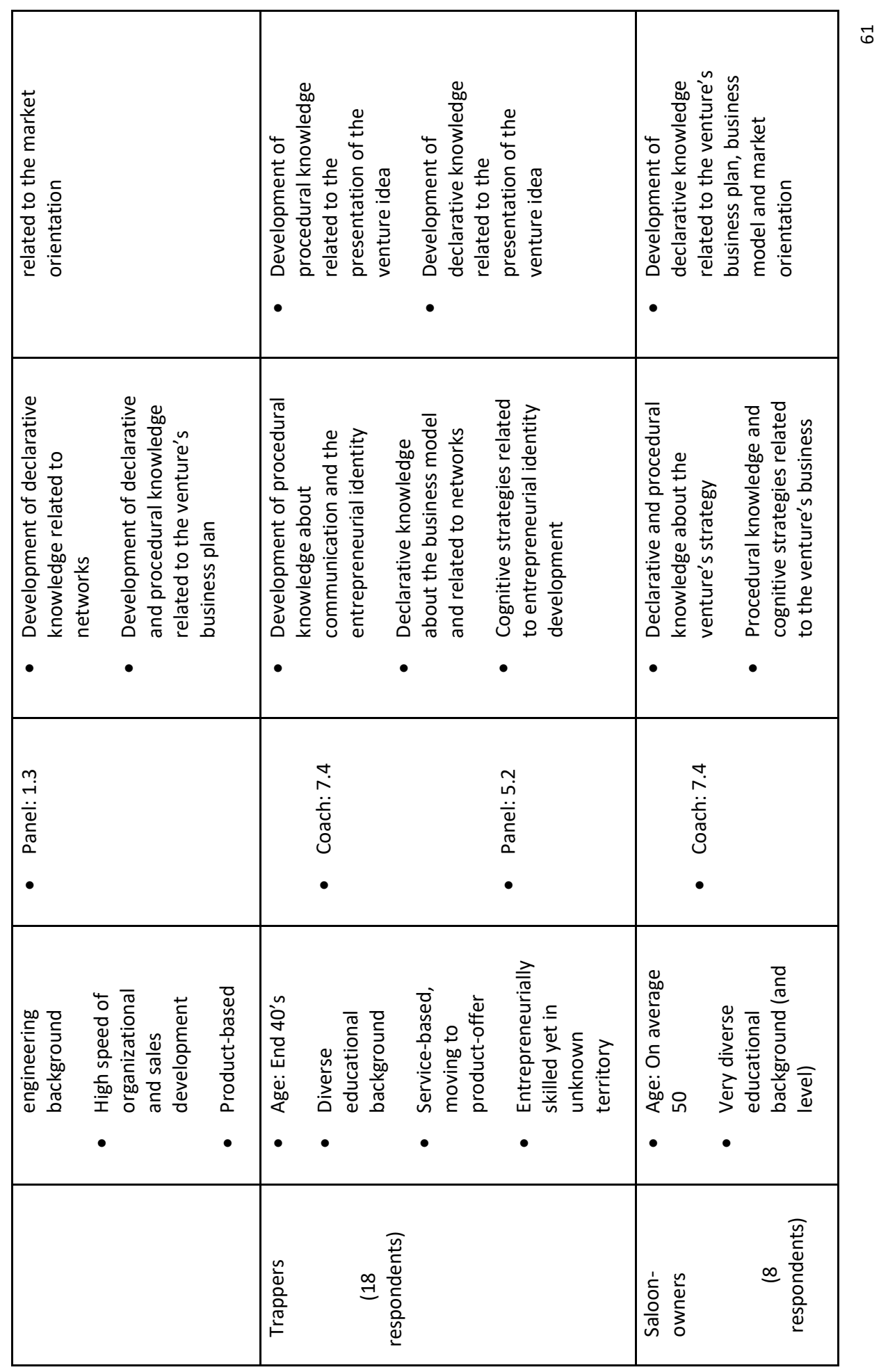




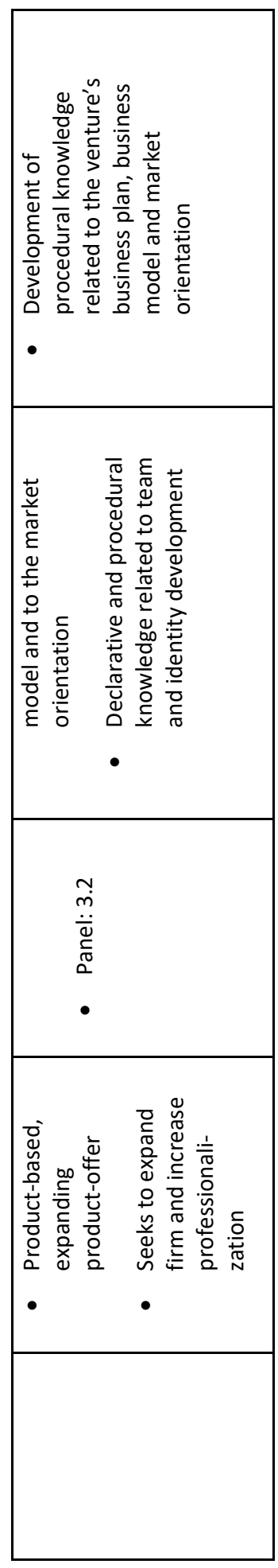




\section{TYPE 1: GREENHORNS - NOVICE ENTREPRENEURS DEVELOPING A SERVICE-BASED VALUE OFFER}

\section{CONDITIONS: EXPERIENCE AND NATURE OF BUSINESS OPPORTUNITY}

18 entrepreneurs from our sample are in the category called 'Greenhorns'. They are novice entrepreneurs, typically in their mid- to late-40's (on average 46 years old) and are characterized by an educational background in arts and social sciences. Only one respondent has an education in the field of natural science. All respondents have on average four years of entrepreneurial experience and 22 years of work experience. That means that they have quite long previous working experience in employment.

Characteristically, these entrepreneurs only recently seriously started to focus on developing their business to a sustainable, long-term enterprise. This does not necessarily mean they have just begun developing a business opportunity. There are entrepreneurs in this sample who have been working self-employed for quite a number of years - in one case, seven years -, but they have had relative stable or even part-time employment incomes from particular source(s) of work. Greenhorns usually develop a service-based business opportunity, most often in the realm of IT services and consultancy, but also in other commercial services and the media \& print sectors.

\section{ACTION/INTERACTION}

Greenhorns report both coaching and panel interactions more often than entrepreneurs of the other three categories. On average, every respondent in this group reports coaching interaction more than ten times and interactions with the panel almost 8 times.

Entrepreneurs in this category seek guidance and structure more than the other types of entrepreneurs. Yet, for them it is not always evident that they are expected to pro-actively 'steer' their coach-interaction. For example, one respondent notes this learning of the previous week in this diary:

R03: "That you don't really profit from coaching if you don't plan it [in your schedule] structurally."

This quote shows the growing awareness of the respondent to be strategic about the coaching - by planning it structurally in his agenda - and also acknowledges the value of 
feedback because the entrepreneur implicitly underlines the 'profit' one can gain from it. This quote is exemplary for the greenhorns' appreciation of targeted feedback instances.

Greenhorns are very affected by panel interaction. It often is received with great appreciation as well as seen as a serious undertaking. An example is given in the following quote which another entrepreneur wrote in his diary:

R01: "Last week was very productive in terms of my first experience of delivering business panel presentation. [..] I got many valuable comments from the panel members and coach. This learning would not be possible if I am literally forced to produce the business concept for the business panel presentation."

This entrepreneur emphasizes the boost in his own activities related to venture development due to the imminent panel presentation. He too acknowledges the value of targeted feedback - not only in terms of valuable feedback, but by providing an incentive to engage in opportunity development.

Respondents in this category repeatedly emphasize the value of the feedback they receive, without being as precise about the content of the feedback solicited and/or given. Both coaching and panel feedback received by these entrepreneurs relates to by a broad variety of thematic learning outcomes.

\section{Consequences}

In general, coaching and panel feedback influences this group strongly in terms of development of the entrepreneurial identity as well as planning and strategizing cognition.

\section{Coaching feedback outcomes}

The effect of coaching feedback on the cognitive development reported by entrepreneurs of the greenhorn type mainly relates to declarative and procedural knowledge about one's entrepreneurial business plan or business model, the venture's strategy as well as one's entrepreneurial identity. In addition, entrepreneurs in this category typically develop cognitive strategies regarding the entrepreneurial identity as a result of coaching. In graph 2-1 we show the thematic learning outcomes reported by 'greenhorn'-entrepreneurs which are related to coaching feedback. 
Graph 2-1: Thematic learning outcomes from feedback associated with coaching reported by greenhorns, in \% of all (inter)actions reported

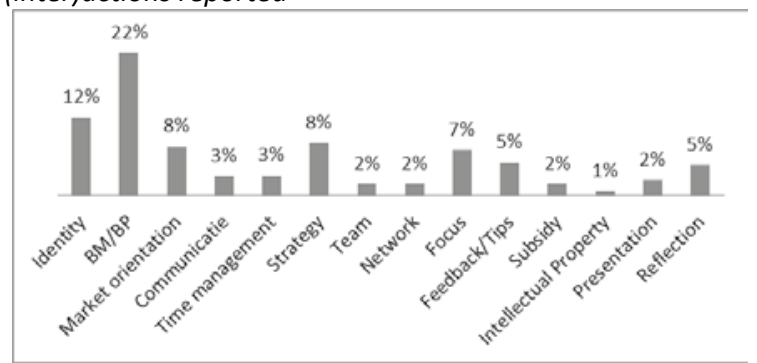

In the case of greenhorns, coaching feedback leads the development of declarative knowledge - for example, in the form of new concepts - and even more often procedural knowledge, such as how to design a business plan. For example, one respondent writes under the heading 'Next steps' in the diary:

R24: "Answering what the added value of my business plan is. The last weeks I have had a brainstorm session with my coach in order to get a better image of [...] the progressive filling of my business plan."

This quote shows how coaching interaction has triggered the respondent to think about and work on his business plan, by means of brainstorming about respectively 'progressive filling' of that plan. The coaches raise awareness for the importance of setting specific goals, working out a venture-set up strategy and finding partners and customers. For example, one entrepreneur in this category wrote in his diary:

R30: "I have planned with [coach]: in 2 weeks I have my added value models ready and we'll discuss them. Also then we make the route for the business plan for the end of April."

This quote shows the development of both declarative and procedural knowledge: the respondent reports his setting of a deadline for 'added value models' which invariably involve learning instances about those models and as well as their design. Also, the entrepreneur mentions the making of a 'route' and this indicates the learning of a process of how to put a business plan together. 
In addition, coaching affects cognitive strategies in terms of identity development, in terms of an acknowledgment of the risks associated with a self-employed lifestyle. Coaches are described as facilitating reflection about personal insecurities and the balance between private life and venture development. For example, one entrepreneur in this category emphasizes the role of the coach in developing an 'entrepreneurial' identity and conflicts that can arise - and financially painful choices - when entrepreneurship is recognized as one's passion:

R33: Last week I had two coaching sessions [...]. These two coaching sessions made things clear for me, but also provided disappointment and a lot of questions that still need to be answered. One thing is for sure, what I am doing now doesn't hold the passion that I need to fulfill what I want. But the main reason for that is simply said money. The money that I need to pay for the mortgage, food and living. This reason keeps me going on doing what I do through the day: work! It is not that I don't have any work to do right now, actually there is work plenty at this time, and more request are coming in. But that also terrifies me. With all work also comes a certain responsibility that doesn't fulfill my passion, it actually distances itself more from my passion and I probably will start to lose focus and interest (while it actually makes money). With all these learnings I am starting to feel lost, lost in a big world where I can't find any time to get my passion on top."

This quote shows that the entrepreneur recognizes the importance of passion for one's profession or more generally income-creating activity. This shows how the respondent's develops his professional identity in terms of an awareness for, and subsequent critical examination of, two seemingly juxtaposed identities, namely passionate entrepreneur versus pragmatic income-earner. The quote is characteristic of how coaching is leveraged for cognitive strategies by 'greenhorn'-entrepreneurs. In this case, the entrepreneur expresses the learning of the cognitive strategy which is best described with the saying 'money cannot buy happiness'.

\section{Panel feedback outcomes}

In this category, panel feedback leads to declarative knowledge and procedural knowledge mainly regarding the entrepreneurs' business model or business plan and his presentational skills. Graph 2-2 gives an overview of the themes that greenhorns mentioned in relation to their leverage of panel feedback. 
Graph 2-2: Thematic learning outcomes associated with panels reported by greenhorns, in \% of (inter)actions reported

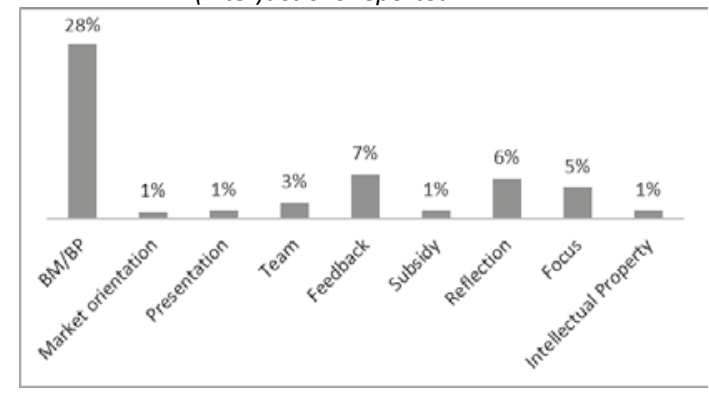

The mechanism of panel feedback entails an examination of the sustainability and profitability of the Greenhorns' business opportunity. For example, one respondent wrote in his diary:

R01: "Last week was very productive in terms of my first experience of delivering business panel presentation. In past I have made several presentations in front of international audience. However this was different and special. It has different dimension. Not just the technology but how to create sustainable business."

This entrepreneur notes how he has learned from panel feedback that a presentation of his business opportunity must contain business aspects. This reflects the development of declarative knowledge - tips on the elements of a good presentation of the business idea -, but also procedural knowledge insofar that the feedback pertains to how a business presentation should be given.

This type of entrepreneur reports general feedback benefits, without specifications about which topic is associated with it. One entrepreneur for example makes a similar observation regarding panel feedback:

R30: "At the business presentation I learned that presenting too much vision and not enough substance does not work for a business panel."

The quote of this respondent shows that he is developing knowledge about the components of a business presentation. In general, the diary entries of 'greenhorn'entrepreneurs show that the panel feedback leads to the development of declarative 
knowledge of how to come up with a viable business idea and which elements such a presentation should contain.

In addition, panel feedback results in the development of procedural knowledge regarding the business plan or business model, and also to cognitive strategies regarding that business plan as well as one's entrepreneurial identity.

This category of entrepreneurs reports the effects of coaching feedback mainly in terms developing procedural knowledge, but also declarative knowledge and cognitive strategies - about the design of the venture's business plan or business model and one's entrepreneurial identity. Panel feedback notably also results in the development of the entrepreneur's declarative knowledge and procedural knowledge related to presentational skill formation. It must be noted that entrepreneurs in the category of greenhorns are not yet very clear about their goals in getting feedback or formulating clear intentions which the other three categories do significantly more.

\section{TyPe 2: CoWboys - NOVICE ENTREPRENEURS DEVELOPING A PRODUCT-BASED VALUE OFFER}

\section{CONDITIONS: EXPERIENCE AND NATURE OF BUSINESS OPPORTUNITY}

12 entrepreneurs in our sample are also novice entrepreneurs but they are different from the first category. They are rather young of age; the average age of entrepreneurs is 36 years. Entrepreneurs in this category typically hold a degree in higher (vocational) education in the natural or engineering sciences. On average, they have two years of entrepreneurial experience and 8 years of working experience.

These entrepreneurs develop their business opportunity mainly in the sector of machinery, (technological) equipment and components. Their venture is set up around a patent or prototype which is (loosely) linked to university or industry research; if not via their own educational track, they are affiliated to experts, professors, or other high-ranked actor in the field of engineering or natural science.

Characteristically, cowboys hold an existing - or pending - patent and have set up their business organization at an amazing speed and become ready to produce value after maximally three years; either on their own or in collaboration with a launching customer. 


\section{ACTION/INTERACTION}

Entrepreneurs in this category mention panel presentations and coaching feedback the least in their diaries, on average 4,85 times; coaching about 3,7 times, and panel presentation and feedback issues only 1,4 times. These are entrepreneurs who are developing their first business idea and who are already making their first sales. Panel presentation (inter)actions seem to be less influential to them, considering that there is hardly any mention of them - or of the panel feedback received - in the logbooks.

Cowboys appear to be the most disciplined regarding their coach and panel meetings. Though they are not as passionate about the feedback they receive as other categories are, they certainly take feedback interactions seriously. As one respondent notes in his diary under the headings 'Next steps':

R35: "Meeting with [coach] on Wednesday, prepare the materials to be discussed."

This quote is exemplary of the cowboys' attitude towards and engagement in feedback interaction. While they report less learning outcomes than the other three categories their diary reports testify their sincerity in maximally leveraging those interaction instances. As another entrepreneur for example notes in his dairy after a panel presentation:

R47: "Panel presentation could go better. But also a little disappointing that the panel members didn't listen well with the exception of [name of one panel member]. They had not prepared sufficiently, such as making the effort to look at my website. However I must admit that I had provided the information of what I wanted to talk about at a rather late time."

This quote demonstrates how cowboys are not only critical about the seriousness of their own efforts but also about those of the panel. This can sometimes even lead to slight disappointment with the way the other party has prepared the interaction instance.

\section{CONSEQuences}

These entrepreneurs report to have shaped their business idea as a result of both feedback mechanisms but without significantly changing their value proposition, collaborations or customer segment(s). Instead, in this category there is a lot of focus on issues of internal organization, collaboration and revenue streams. 


\section{Coaching feedback outcomes}

Cowboys are by far more focused on coaching than on panel feedback. They report cognitive learning outcomes in terms of the development of a market orientation and their own entrepreneurial identity, as well as time management and venture team aspects. Graph 2-3 depicts the distribution of thematic learning outcomes associated with coaching feedback.

Graph 2-3: Feedback leverage thematic learning outcomes associated with coaching reported by cowboys, in \% of (inter)actions reported

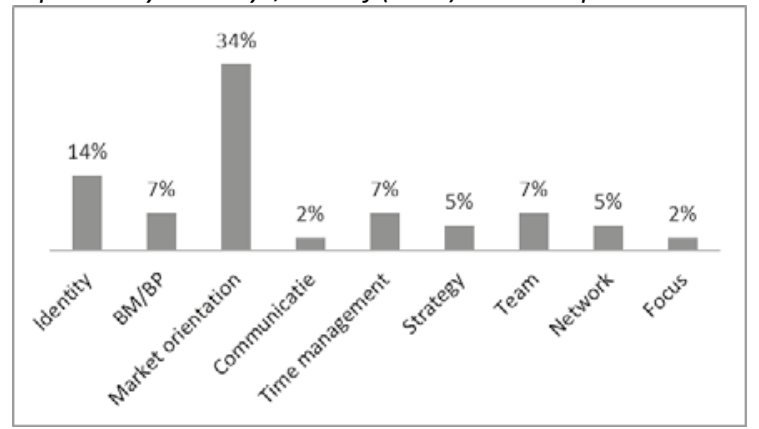

Respondents in this group are affected by coaching feedback in very specific, marketoriented aspects such as sales, marketing and customer relations and which are linked to the development of procedural and declarative knowledge. For example, one respondent writes:

R48: "How do I get a follow-up assignment, and new acquisition? Discussed this with my coach: I will call the [local, formal industrial network] if I can present myself."

This entrepreneur reports a very concrete effect of coaching feedback, namely the development of declarative and procedural knowledge about network, in this case how to present one's venture to potential customers in specific networks he simultaneously is learning about. It is a typical example of the way cowboys are affected by targeted feedback, namely in terms of very specific actionable intentions - which is in contrast to the more general that the diaries of greenhorn-type entrepreneurs contain. 
While market orientation and network development are of major concern to entrepreneurs of the category 'cowboys', they also mention the value of coaching feedback regarding team issues, as shows the following quote:

R35: "Some progress is made on the professionalism of our organization. I have a quite good feeling how to implement this and shared these ideas with two other shareholders [...]. Coming Wednesday I will discuss this with [the coach] as well. The plan is to discuss this with all share-holders after the summer holidays in September and start with the implementation thereafter."

This entrepreneur reports how coaching feedback for him is mainly relevant for the professionalization of his venture's organization and the role of shareholders in this. The quote shows how the development of both declarative and procedural knowledge about certain strategic issues are combined in the cognitive learning outcomes of cowboys as a result of coaching feedback.

Entrepreneurs of this category also leverage coaching feedback for identity development differently than greenhorn-entrepreneurs. While greenhorns perceive the coach as a guide, for cowboys the role of the coach is that of 'sparring partner'. As exemplified in the following diary quote, identity development is seen much more in terms of improving specific entrepreneurial or business skills:

R23: "Had a constructive coach meeting regarding certain business issues. Must make an extra step in clarifying the unique selling points."

As we see, this entrepreneurs reports coaching feedback regarding identity development in terms of business skill development, specifically his presentational and marketing skills. This shows that cowboys' reports are related to the development of both declarative and procedural knowledge about business management skills, as opposed to the greenhorns' emphasis on reflection about entrepreneurial values such as risk-taking or autonomy.

\section{Panel feedback outcomes}

In terms of the panel feedback mechanism, entrepreneurs of this category mainly report the development of procedural knowledge regarding the business model or business plan of the venture and market-orientation - but only mention this type of feedback very 
scarcely. Graph 2-4 shows which thematic learning outcomes cowboys reported in relation to panel feedback leverage.

Graph 2-4: Feedback leverage thematic learning outcomes associated with panel reported by cowboys, in \% of all reported (inter)actions

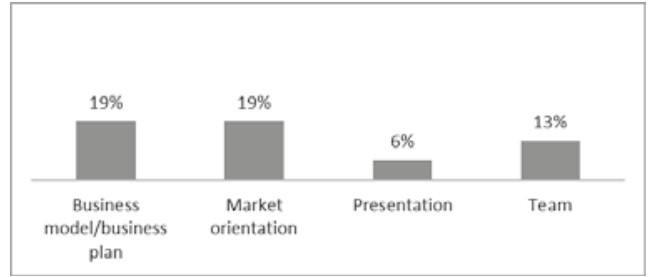

Cowboys use panel feedback to 'test' their business plan, value creation logic or market orientation. Like coaching, cowboys leverage panel feedback by translating the feedback to specific points of attention. For example, one respondent wrote in his diary:

R23: "Had a panel presentation with triggering feedback. How to approach the telecom market: it might not be good to approach the big companies first."

This quote shows the entrepreneur's development of procedural knowledge, which is reflected in his note that it 'might not be good to approach the big companies first'. It is a typical demonstration of the cowboy-entrepreneurs' pattern of panel feedback outcome: it is not mentioned often but when it does is mentioned it is taken very seriously by the respondents of this category and related to the development of procedural knowledge.

In fact, in the case of cowboys, panel feedback results mainly in the development of procedural knowledge, and hardly in that of declarative knowledge and cognitive strategies. Another respondent is also very concrete and serious about the cognitive learning outcome of panel feedback:

R48: Today [my business partner] and I held a panel presentation. I noticed how motivated the panel members are (2 people), and we received feedback which we found very meaningful. The most valuable for me was the business assessment Venture Lab with which on the one hand we can design our own professional presentation model based on those compounds and on the other hand we can integrate the marketing plan in it (business model). We hope to show this more clearly in the next panel presentation. 
The respondent reports how the panel feedback will help to make a 'professional' presentation-model, and indicates that he has learned how to integrate the marketing plan into the business model. We see that the entrepreneur immediately relates the panel feedback to concrete intentions, while reporting the development of procedural knowledge in terms of because how to use a specific presentation model for the integration of the venture's marketing plan.

We observed that cowboys report cognitive learning quite differently than the other categories. They report significantly less thematic categories than entrepreneurs of the other three types, and almost exclusively report the development of procedural knowledge. And they are very concrete in the formulation of next steps based on that feedback.

For this type of entrepreneur, cognitive development as a result of coaching occurs mainly in terms of declarative and procedural knowledge. The results of targeted feedback mechanisms are very specifically described and about venture development in terms of business model or business plan. Coaching feedback also related to entrepreneurial identity development however unlike greenhorns these entrepreneurs are concerned with becoming more 'business-like', also seen in their concern with planning and strategic skills (mainly team and time management related). Panel feedback is scarcely mentioned and relates to the development of procedural knowledge related to the venture's business model or business plan, the entrepreneur's market orientation, his presentational skills and team issues.

TYPE 3: TRAPPERS - EXPERIENCED ENTREPRENEURS MOVING FROM SERVICE-BASED VALUE OFFER TO PRODUCT-BASED VALUE OFFER

\section{CONDITIONS: EXPERIENCE AND NATURE OF BUSINESS OPPORTUNITY}

18 entrepreneurs in our sample are categorized as trappers. Trapper-entrepreneurs have an existing venture, typically based on a service-offer, and aim to develop another, product-based opportunity.

In this category, entrepreneurs characteristically work in the sectors of Commercial services \& Supplies and IT-services -consultancy. They are accustomed to being selfemployed, however work as service providers and therefore unfamiliar with production chains and up-front investments. 
Trappers are diversely educated; educational backgrounds include the humanities, social sciences, engineering and natural sciences. On average, a 'trapper' is 50 years old and has 10 years of prior entrepreneurial experience. This type of entrepreneur also has the highest amount of work experience in employment: on average 16 years.

\section{ACTION/INTERACTION}

With respect to coaching and panel (inter)action reported in the diaries, trappers report them on average 12 times, respectively 7 and 5 times (coaching and panel interactions). This service-experienced category of entrepreneurs mentions both feedback mechanisms second most often.

This category of entrepreneur changes coach or express dissatisfaction with the coach more than the other types, for example by reporting more critical notes about the coach not being the right person. As one trapper-entrepreneur noted in his diary:

R28: "Switched coach because of need for more practical support and experience."

in terms of not being knowledgeable enough in terms of entrepreneurial experience or lacking expertise in the sector in which they aim to develop a product-based business opportunity.

\section{Consequences}

In the diaries of this type of entrepreneur we identified many different thematic learning outcomes as an effect of targeted feedback. Both coaching and panel feedback are reported the second most often, only surpassed by the greenhorn-entrepreneurs.

\section{Coaching feedback outcomes}

The thematic learning outcomes of coaching are related to development of the business model or business plan, and the entrepreneurs' identity. Other important coaching effects relate to learning how to focus, as well about time planning, strategy and the entrepreneur's market orientation. The broad variety of topics reported is characteristic of both trappers and greenhorns. Graph 2-5 displays the thematic learning outcomes that trappers report which result from coaching feedback. 
Graph 2-5: Feedback leverage thematic learning outcomes associated with coaching reported by trappers, in \% of (inter)actions reported

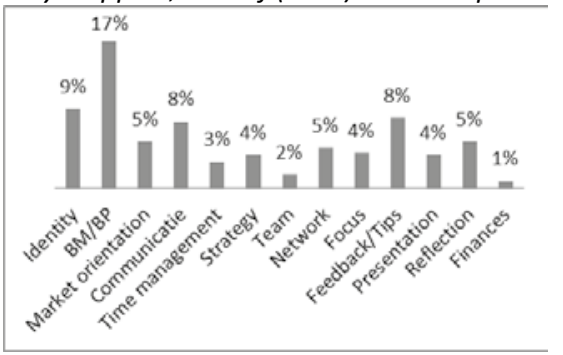

Trappers frequently seek advice on how to set up a business model, for example for adding a new or different value offer to their existing venture. Yet, this is quite different from their existing business model because now they face value creation via a product, and it leads to a lot of new learnings. For example, one respondent writes in his diary:

R19: "After an excellent session with my coach l've learned that a profit model might even work with given everything away for free! Busy now working out all variations."

The entrepreneur points to his learning of business modeling and the different ways in which value can be created. This reflects the development of declarative knowledge, namely the concept of social entrepreneurship in the form of non-profit business models.

Trappers perceive coaching as affecting the way value is created in the face of changing from an already existing service-offer or adding to it a product-offer which is unchartered territory for them. They are remarkably cautious about what could go wrong and weary of the time- and energy-consuming path of product development. This becomes particularly salient in the way they leverage coaching feedback for entrepreneurial personality development, as the following quote from the diary exemplifies:

R15: "[Learnings o]n personal level after session with coach: Bring the [business opportunity] more towards my personal ambition and role. This will make it more valuable for [the existing venture] and more sustainable next to all the other activities that require attention."

The quote shows that the entrepreneur appreciates the sparring partner he sees in the coach, but it is also of importance to him that the coach is available and effective. This 
quote reflects how procedural knowledge and cognitive strategies are developed at the same time; in this case the process of developing a business opportunity - bringing it towards his personal ambition and role - and reflection on that this can be helpful for his business activities in general, by making it more sustainable in the long run.

Respondents who we categorized in the type 'trapper' report the effects of coaching feedback in terms of development of declarative knowledge about business model and networks, as well as the development of procedural knowledge about communication and presentation skills, and procedural knowledge/cognitive strategies regarding the entrepreneur's identity.

It must be noted that trappers are experienced entrepreneurs and can use that expertise to avoid 'rookie' mistakes in opportunity development. They are critical and/or demanding regarding coaching quality; as already mentioned, entrepreneurs of this type more often change coach than entrepreneurs in the other categories.

\section{Panel feedback outcomes}

In terms of effects of the mechanism of panel feedback, entrepreneurs of the trappertype appreciate the panel's critical feedback and they are willing to reflect upon it as well as put it to practical use. Graph 2-6 shows the distribution of thematic learning outcomes associated with panel feedback reported by this type.

Graph 2-6: Feedback leverage thematic learning outcomes associated with panels reported by trappers, in \% of reports

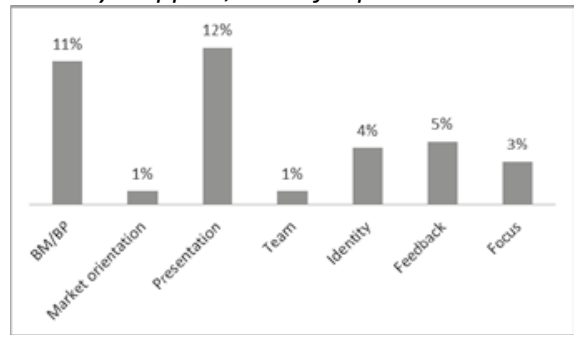

In general, entrepreneurs in this category appreciate panel feedback more than entrepreneurs in the other categories. We found that this is the case because they can test their envisioned value creation logic. For example, one respondent says the following about feedback received in his panel presentation: 
R19: "I need to define my business model more thoroughly as I thought before. This is a conclusion of listening to the 'money suppliers' in the panel."

This quote shows how the entrepreneur learning from panel feedback about how to design his business model and reflects the development of procedural knowledge. The emphasis that entrepreneurs in this category put on searching for feedback on details of their business opportunity is characteristic for this group. While entrepreneurs of this type have experience in developing a business opportunity they also know that it is important to work on improving one's communicational skills. For example, one respondent notes in this diary:

R04: "I gave my second VLT panel presentation: it was pleasant, good training, but learned nothing new from the panel (except for how to better communicate my ideas)."

The entrepreneur, while reporting that he 'learned nothing' new, actually reports a learning outcome, namely how to better communicate his ideas which reflects the development of procedural knowledge.

To a lesser extent, we found that panel feedback also leads to the development of declarative knowledge and - unlike the other categories - to the development of cognitive strategies thematically related to the presentation of their business idea and their entrepreneurial identity.

In general, this type of entrepreneur reports a wide range of thematic learning outcomes. Those learning outcomes reflect cognitive development mainly terms of procedural knowledge and cognitive strategies regarding the venture's business model, for example how to make their business opportunity attractive for relevant market parties - such as collaborative partners, potential subsidy distributors - but also how to set up a team. Panel feedback leads to the development of procedural knowledge (and also declarative knowledge and cognitive strategies, though much less) on a more restricted number of themes, related to how to design and present the business opportunity, how to focus a business plan or their own identity development. 
TYPE 4: SALOON-OWNERS - EXPERIENCED ENTREPRENEURS EXPANDING AN EXISTING PRODUCT-BASED VALUE OFFER

\section{CONDITIONS: EXPERIENCE AND NATURE OF BUSINESS OPPORTUNITY}

The fourth category of entrepreneurs also consists of experienced entrepreneurs. They characteristically seek to enter a new market with an existing product or want to develop a new product in addition to the already existing product. They are slightly older on average than entrepreneurs in the other three categories, namely 49 years.

Saloon-owners have the most entrepreneurial experience of all categories - on average 12 years - however less work experience in employment than the third category: 13 years on average. In terms of education background they are very diverse; in this category we find more entrepreneurs than in the other categories whose highest degree is in secondary education. In this category, entrepreneurs with an educational background in social sciences are higher educated (academic degree) than those with a background in natural sciences or engineering (higher vocational training). The sectors these entrepreneurs operate in are typically those of Commercial Services \& Supplies, Software \& IT services and to a lesser extent also Machinery, Equipment \& Components.

\section{ACTION/INTERACTION}

Entrepreneurs in this category report an average of 10 instances in the diary which are related to coaching and panel feedback mechanisms. Coaching feedback interactions however get considerable more attention than those of the panel feedback; they are reported respectively 7 and 3 times on average.

Saloon-owners have prior entrepreneurial experience, they display a similar, experienced-based critique on the suitability of the coaching as trappers do. Yet, this type seems to be milder in their critique; they don't change coaches like trappers do. This could be due to a greater satisfaction with the status quo of their company than their service-based counterparts and to a less extreme change of their business; after all, going from serviceoffer to product-offer requires more novel learning experiences than the development of another product offer. 


\section{Consequences}

It appears that saloon-owners have gotten rather 'wrapped up' in day-to-day business activities and seek to use targeted feedback to reassess their venture's long-term, strategic vision about value creation and delivery.

\section{Coaching feedback outcomes}

The effects of coaching pertain to themes of identity and business model/plan development as well as issues concerning his market orientation. There are also a variety of other themes that are mentioned. Graph 2-7 shows the saloon-owners' thematic learning outcomes as a result of coaching feedback.

Graph 2-7: Thematic learning outcomes as a result of coaching feedback. Reported by saloon-owners, in \% of all reports of this type

In this category, entrepreneurs are concerned with the planning of the company's future, but also what that means for themselves. For example, one respondent in this category reflects on the lack of time for more reflection about the future of his business, and the role of the coach in this:

R44: "This week I had a meeting with my coach for the first time and I liked it a lot. What I learned most is that I don't know yet precisely which direction I want to go. Do I want to start a new business, or do I want to improve the existing one. Secondly, I am profiting a lot from the knowledge that my coach has and that I can immediately apply in my own business."

This entrepreneur reports that he is learning about where he wants to take his company and that the coach is helping with that because he doesn't know yet which direction 
he wants to go'. The quote reflects the development of cognitive strategies because the respondent points out explicitly that he is learning how to learn - namely, to determine whether his existing venture is suitable for the development of new business opportunity or not.

Respondents of this category perceive a coach as a general sparring partner for growth- and organizational questions. In that sense, coaching is beneficial to reflect on the entrepreneur's (changing) role in his company which is more mature in comparison to the state of venture development of the other three categories; for example, planning in time for reflection.

Equally important for saloon-owners is strong and structured interaction with their environment, in terms of new impulses coming from a new or different product, or entering a new market. They are particularly concerned with the identification and acquisition of more or different customer segments and the best way to deliver value to these segments. For example, one respondent in this category wrote in his diary:

R29: "With my coach I discussed the effort I make for new customers. We tried to define groups and the first thing I need to do is get in contact with relevant people from that groups to avoid to sell to each individual client and try to make it interesting for the groups to buy together."

This respondent points to the need to redefine his selling activities by rethinking the way he sees customers; namely, instead of serving one customer at a time, move towards group-oriented sales activities. This reflects the development of procedural knowledge in terms of how to do sales. Another instance of procedural knowledge - in combination with declarative knowledge - is given by the following respondent when he reports the following thoughts under the heading 'Learnings' in his diary:

R07: "During the 2 coach session new way of thinking about your business and the structure. We completed the business model in an I-pad application and it was very useful. So I learned to work with this new business application as software tool and to complete it according the business model generation process/book." 
This quote exemplifies the benefits of coaching for the entrepreneurs' professionalization in terms of specific business development, characteristic for coaching effects reported by respondents of this type.

\section{Panel feedback outcomes}

Panel feedback triggers reflection and even different views among saloon-owners. The results shown in graph 2-8 gives an overview thematic learning outcomes as a result of panel feedback reported by entrepreneurs of this category.

Graph 2-8: Feedback leverage thematic learning outcomes associated with panels reported by saloon-owners, in \% of total reports of panel interaction in this category

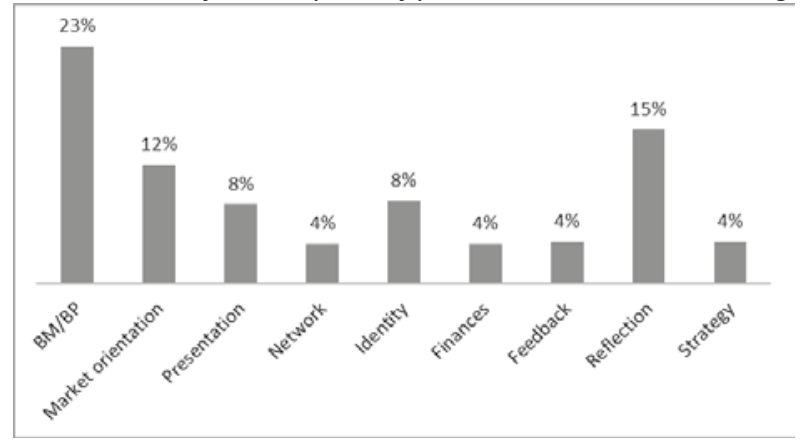

We see that the thematic learning outcomes of business model/business plan, market orientation and reflection dominate panel feedback leverage by saloon-owners. The effect of feedback is described as facilitating a change in the entrepreneur's perspective on his company. As one respondent notes in his diary:

R46: "Outcome of panel presentation: Way of thinking about the company/market opportunities is very personal. When you are not used to think 'big', you need assistance for this (if that is the goal for the company/product). [The product idea] has a great opportunity, but needs an organization for this."

The entrepreneur reports how panel feedback is leveraged for better business model development, in particular how to prepare the internal organization in order to match the scaling of the product. This indicates the development of procedural knowledge linked to the venture's organization. 
Panel feedback is used by entrepreneurs in this type to improve the business model, with an emphasis on customer value creation. One respondent explicitly addressed this issue in the following way:

R13: "This afternoon I did my first panel presentation. I was to much focussed on the technical aspects of [the business opportunity]. The panel missed my commercial paragraph and an enumeration of other possible technical solutions. They also advised me to investigate possible other technical applications."

The entrepreneur reports his learning of a model, which reflects the development of declarative knowledge - namely, that model. The quote also shows that experienced entrepreneurs resemble novice ones in the way that they are not necessarily focused on customer needs. The panel feedback effects show that even experienced entrepreneurs who run a successful business benefit from targeted feedback in terms of reflection about their new business opportunity, especially regarding the entrepreneurs' customer- and market orientation.

For this category, coaching feedback characteristically leads to a wide variety of thematic learning outcomes, mainly occurs in terms of procedural knowledge, or combined declarative and procedural knowledge, and sometimes also in terms of cognitive strategies. The effect of panel feedback on cognitive development is mainly reported in terms of declarative and procedural knowledge regarding the venture's business plan or business model, as well as the entrepreneur's market orientation.

Interestingly, we observed that saloon-owners (similar to cowboys) have a tendency to formulate goals and intentions precisely; with regards to expectations from coaching and panel feedback as well as a result of it. Saloon-owners, just as cowboys, are more at ease and further along with the product development process than entrepreneurs of the other two types. 


\subsection{CONCLUSION}

Above we described the effect of coaching and panel feedback on the cognitive development of entrepreneurs. Based on the results of our analysis, we draw two major conclusions. One, we can distinguish between different categories of entrepreneurs with respect to cognitive development. Second, that targeted coaching and panel feedback mechanisms have a different effect on the cognitive development of entrepreneurs. Each of the conclusions are described below.

TAXONOMY OF ENTREPRENEURS: HOW ENTREPRENEURIAL EXPERIENCE AND TYPE OF BUSINESS OPPORTUNITY AFFECT TARGETED FEEDBACK OUTCOMES

We distinguish between four categories of entrepreneurs that we labelled, respectively, 'Greenhorns', 'Cowboys', 'Trappers' and 'Saloon-owners'. Appendix 2 shows the comparison of the four types of entrepreneurs we identified among the respondents in our sample in terms of thematic learning outcomes as a result of coaching respectively panel (inter)actions.

These four categories differ along two characteristics: prior entrepreneurial experience and the type of opportunity which is developed by entrepreneurs (in terms of product or service). Entrepreneurs in these categories show distinctive effects of cognitive development as a result of coaching and panel feedback mechanisms. Below we present these conclusions in more detail.

\section{THE ROLE OF ENTREPRENEURIAL EXPERIENCE}

Our findings show that novice entrepreneurs report different cognitive learning outcomes than experienced entrepreneurs. Entrepreneurs who have relatively little or no prior entrepreneurial experience - typified as greenhorns and cowboys - report the effects of coaching feedback in terms of development of declarative knowledge more than experienced entrepreneurs, particularly regarding their identity and the making of their business model or business plan. The experienced entrepreneurs - trappers and saloonowners - report the effect of coaching feedback for development of procedural knowledge and cognitive strategies than novice entrepreneurs, in particular the combination of procedural knowledge and declarative knowledge development. 
Another difference between novice and experienced entrepreneurs is that experienced entrepreneurs report the changing of coaches in their diaries while novice entrepreneurs don't appear to be changing coaches, or at least do not mention it. This indicates that experienced entrepreneurs more often than novice entrepreneurs experience a mismatch between their own expectations and the coaching feedback they receive.

\section{THE ROLE OF THE VENTURE'S VALUE OFFER}

Another important factor is the business opportunity which is developed by the entrepreneurs. Both greenhorns and trappers report a considerably larger variety of thematic learning outcomes than cowboys and saloon-owners, with an emphasis on themes of development of focus in and presentation of their business idea. Also, both types leverage panel feedback for cognitive learning largely in terms of declarative knowledge development. While trappers can draw on their existing expertise, it appears that this expertise does not per se facilitate a shift to a product based, novel business opportunity.

A possible explanation is that their background in service-based opportunity development makes the move to product-based more challenging, resulting in more learning instances. Prior experience in service-based value creation generates an expertise which is unlike that of entrepreneurs who develop a product-based value offer.

The value offer of trappers and greenhorns distinguishes itself from that of cowboys and saloon-owners in that it is typically not in a manufacturing sector - such as electronics, machinery and component - but mainly in the realm of ICT, for example software. That means that their value proposition is less 'material' or scalable than that of cowboys and saloon-owners and yields a situation similar to that of greenhorns.

As for cowboys and saloon-owners who characteristically develop a manufactured product we found similarities in the effects of targeted feedback. For example, panel feedback outcomes in terms of the development of one's market orientation is reported almost exclusively by cowboys and saloon-owners.

We conclude that prior entrepreneurial experience and the nature of a business opportunity affects the way in which entrepreneurs' cognitive development is affected by targeted feedback mechanisms. 


\section{COMPARING THE EFFECTS OF COACHING AND PANEL FEEDBACK ON COGNITIVE DEVELOPMENT OF}

\section{ENTREPRENEURS}

Both feedback mechanisms yield different effect per type of entrepreneur. Those are described below per feedback mechanism, then compared and contrasted.

\section{COACHING FEEDBACK}

The results show that coaching feedback affects cognitive development outcomes on a broader variety of themes than panel feedback, and leads to considerably more development of cognitive strategies than panel feedback.

In the case of greenhorns, coaching results mainly in the development of procedural knowledge and to a lesser extent declarative knowledge about their venture's business plan. The development of procedural knowledge also occurs with respect to their entrepreneurial identity, in terms of their own entrepreneurial attitude and behavior. This type also develops cognitive strategies related mainly to identity development, and to a lesser extent to business modeling or business planning.

As for the cowboys, coaching feedback predominantly results in the development of declarative and procedural knowledge with their market orientation, and to a lesser extent with respect to their entrepreneurial attitude and behavior. The development of cognitive strategies are hardly affected by coaching feedback.

In the case of trappers, coaching feedback leads to learning linked to more thematic categories than in the other three categories. Notably, it leads to the development of procedural knowledge about how to communicate with external parties and the development of their entrepreneurial identity, but also to declarative knowledge about the business model and the entrepreneur's network. They also develop cognitive strategies with regards to their entrepreneurial identity, but to a much lesser extent.

For saloon-owners', coaching feedback predominantly results in the development of combined declarative and procedural knowledge about their venture's strategy, respectively combined procedural knowledge and cognitive strategies related to their venture's business model or their market orientation. 


\section{Panel feedback}

Regarding panel (inter)actions, all categories report feedback leverage mainly in terms of declarative knowledge and procedural knowledge related to their business plan and/or business model. In fact, the development of declarative knowledge is reported more often as a result of panel feedback than of coaching feedback. Thematically, entrepreneurs report presentational skills considerably more in the case of panel feedback than coaching feedback.

Greenhorns report the results of panel feedback mainly in terms of the development of declarative knowledge about the venture presentation itself, but also procedural knowledge and cognitive strategies regarding the entrepreneurs' identity and their business plan. This type develops cognitive strategies (or meta-cognitive development) more often as a result of panel feedback than of coaching feedback.

Cowboys are affected by panel feedback mainly in terms of procedural knowledge about their business model, business plan or market orientation development. The other two cognitive learning outcomes are not often reported. However, the development of cognitive strategies is still more often reported than in the case of coaching feedback and related to issues of team development.

For trappers, the main panel feedback outcome is the development of procedural knowledge and to a lesser extent declarative knowledge. Thematically, both cognitive learning outcomes relate to the presentation of their venture idea. Other cognitive learning outcomes are considerably less reported.

Saloon-owners report the effects of panel feedback mainly in terms of the development of declarative and procedural knowledge. Both are more often mentioned as a result of panel feedback than of coaching feedback and relate to the entrepreneur's business plan, business model and market orientation. This type hardly mentions cognitive strategies as an outcome of panel feedback.

\section{OUTCOMES OF TARGETED FEEDBACK MECHANISMS: SIMILARITIES AND CONTRASTS}

While both coaching and panel feedback frequently affect the entrepreneur's procedural knowledge - also called knowledge organization - they differ regarding the other two cognitive learning outcomes: learning of declarative knowledge and cognitive strategies. 
Our results show that coaching feedback affects the development of procedural knowledge and cognitive strategies (meta-cognitive development) regarding the venture's business model or its business plan and to the entrepreneur's reflection on and cognitive development of his own entrepreneurial identity. Panel feedback affects more learning of declarative knowledge; in particular regarding the theme business model/business plan.

That the development of cognitive strategies (or meta-cognitive development) is more often related to coaching feedback instead of panel feedback might be related to the fact that coaching sessions were much more frequent and regular than panel interactions. The latter only occurred once every three months while coaching was a weekly activity. More personal coaching (inter)actions provide a setting inductive to intimate and trust-based relationship, thus possibly enhancing (openness to) cognitive learning by the entrepreneur.

We also found that panel feedback has an absolute and relative more powerful effect on the thematic learning outcomes of business plan and business model development, as well as the entrepreneur's presentation of his venture idea, particularly in the development of declarative and procedural knowledge. Solely trappers - who also report to change the coach more than the other three types - report all types of learning outcomes more often as a result of coaching feedback, absolutely and relatively.

We conclude that on the one hand, coaching feedback results in a broader variety of thematic learning outcomes, and in absolute terms leads to more development of cognitive strategies than panel feedback. On the other hand however, panel feedback is more 'efficient' for the development of cognitive strategies in the case of greenhorns and cowboys, and for declarative and procedural knowledge for entrepreneurs categorized as saloonowners.

We also conclude that while in absolute numbers cognitive learning outcomes are more often reported as a result of coaching feedback, they are relatively more reported as a result of panel feedback. 


\subsection{DisCUSSION AND CONTRIBUTION}

In this section, we discuss the three conclusions presented above in the light of their contribution to the field of entrepreneurship.

\section{TAXONOMY DEVELOPMENT}

The taxonomy presented in this study enables a segmentation of entrepreneurs according to their differing 'response' to targeted feedback mechanisms in terms of cognitive development. St-Jean \& Audet (2012) showed that coaching feedback mainly results in procedural knowledge (knowledge organization) and declarative (verbal) knowledge). Our results confirm their findings, yet also yield a more nuanced picture of targeted feedback mechanisms and their effect on the cognitive development of different categories of entrepreneurs.

In particular the role of prior entrepreneurial experience is commonly recognized as a decisive variable in cognitive processes in entrepreneurial cognition (Baron \& Ensley, 2006; Grégoire et al., 2010). Entrepreneurs make decisions based on their cognitive ability to 'connect the dots', to bring together bits of previously unconnected information, in order to create novel value (Baron \& Ensley, 2006). This ability is affected by the entrepreneur's prior knowledge (Grégoire et al., 2010). Human capital in the form of entrepreneurial experience matters during business opportunity identification, preparation and exploitation (Smith, 1967; Davidsson \& Honig, 2003; Haynie et al., 2009). For example, expert entrepreneurs deal with information differently than novice entrepreneurs (Dew et al., 2009).

Results of this study also yield evidence for a correlation between the type of opportunity or value offer - service-based or product-based - and the way in which targeted feedback affects an entrepreneurs' cognitive development. Regarding the role of the intrinsic characteristics of an entrepreneurial opportunity, this has been pointed out by Grégoire \& Shepherd (2012) who criticize that "differences among opportunities have been largely ignored". The authors suggest that differences in business opportunities are not only based on cognitive attributes but also on differences in the nature of business opportunities, which parallels the finding of this study. 
Our results provide empirical underpinnings for the role that the nature of a value offer plays in entrepreneurial cognitive development, but also advances theory-building on the effects of intrinsic opportunity differences on entrepreneurial cognition.

\section{COACHING VERSUS PANEL FEEDBACK EFFECTS ON COGNITIVE DEVELOPMENT}

The results of this study provide a yet lacking distinction between the effects of two different targeted feedback mechanisms on entrepreneurial cognitive development, coaching and panel feedback.

We found a complementary effect of those two feedback mechanism in terms of 'pulling' or 'pushing' method of feedback (Cull, 2006). The differences between coaching and panel feedback outcomes parallel the differences between pull and push effects of feedback as described by Cull (2006). The author describes the 'push' method of feedback as drawing someone in the direction of the 'right' conclusion by being explicit about it, while the 'pulling' method helps entrepreneurs to develop their meta-cognition.

Panel feedback notably leads to the acquisition of new information, concepts or theories which has the effect of 'pushing'. 'Pushing' feedback relates to "the ability to offer stimulation; to offer creative ideas, challenges, knowledge, success stories, models and tools, leading-edge thinking and wisdom". (Cull, 2006: p. 10). That type of knowledge is associated with 'declarative knowledge' and, as our findings show, happens more often in panel feedback instances than in coaching feedback. For example, the categories of 'Greenhorns', 'Cowboys' and 'Trappers' report the learning of presentational skills in relation to the panel feedback mechanism which is an important skill in business development and for investor attractiveness (Chen et al., 2009; Cardon et al., 2009). This is a valuable 'pushing' effect of panel feedback.

Coaching, on the contrary, leads to what is called the 'pull' effect: The 'pulling' effect of feedback is associated with coaching support by listening, asking the right questions and drawing out the mentee's own answers to problems. We show that feedback mechanism of coaching functions mainly in terms of the 'pulling' method which helps entrepreneurs to develop their meta-cognition, such as reflection and the learning of an entrepreneurial, market-oriented mindset. This 'pulling' is also related to a more complex forms of cognitive learning, in terms of cognitive strategies. In this study, we find that while the 'pushing' is 
more frequently achieved by panel feedback, the 'pulling' is mainly affected by coaching feedback, in particular in terms of meta-cognitive development or 'deeper-level' learning.

This reflective, meta-cognitive (Haynie et al., 2012; St-Jean \& Audet, 2012) or 'deeperlevel' entrepreneurial learning (Krueger, 2007) is associated with the ability to reflect upon, understand and control one's learning, for example by means of conditional knowledge: when and why to use strategies (Schraw \& Dennison, 1994), which is particularly relevant for in the case of business opportunity development. For example, Haynie et al. (2010a) who examined the nature of a situated metacognitive mindset of entrepreneurs, argue that metacognition can be enhanced through training and that it is important in achieving desirable outcomes from entrepreneurial actions.

Baron (2014) defined the development of accurate metacognition as one of the intriguing questions meriting more attention by entrepreneurship scholars. Our results provide empirical evidence that coaching and panel targeted feedback mechanisms lead to the development of (meta)cognitive skills, and that coaching is a more effective cognitive mechanisms metacognitive learning than panel feedback. In doing so, this study contributes to more insights into entrepreneurial metacognition and its development.

On a more practical note, the segmentation of entrepreneurs according to cognitive learning outcomes yields in combination with the insights into the pull and push effects of coaching respectively panel feedback provide valuable tools for planning and implementing feedback instances in incubation/acceleration programs. Our results also yield insights into the moderating role of the type of business opportunity and thus present support systems a way to reflect on the optimal coaching and mentoring matching and content.

\section{TARGETED FEEDBACK MECHANISMS AS FORMS OF SOCIALLY SITUATED COGNITION}

Thirdly, this study contributes to understanding the role of socially situated cognition in entrepreneurship. The conceptualization of entrepreneurship as a socially situated cognitive phenomenon has come to be widely recognized in entrepreneurship studies (Mitchell et al., 2011; Mitchell et al., 2014; Clarke \& Cornelissen, 2014).

Socially situated cognition understands entrepreneurship as action-oriented, situated, embodied and distributed (Mitchell et al., 2011). In particular, the role of language has received attention in studies which examine at the socially situated cognitive nature of opportunity development (Mitchell et al., 2014; Clarke \& Cornelissen, 2014). 
This study yields insights into distributed and embedded cognitive processes in entrepreneurship, by its focus on the effects of interactive feedback processes on entrepreneurial cognitive development. Entrepreneurs make sense of venture-related issues by engaging in feedback interaction which leads to the development of declarative knowledge, procedural knowledge or cognitive strategies. The development of these forms of knowledge or cognitive structures is aimed at creating legitimate economic value in the form of successful development of a business opportunity.

Meaningful, verbal feedback during coaching and panel interaction contributes to establishing the venture's legitimacy. Legitimacy plays a key role in successful opportunity development (Zimmerman \& Zeitz, 2002; Navis \& Glynn, 2010; Werven et al., 2015). It is via discursive interaction that entrepreneurs express and shape thoughts, actions and desires, and learn how to create a legitimate and successful business opportunity (Werven et al., 2015).

Our findings show how different, socially situated and embedded targeted feedback mechanisms affect the cognitive development of entrepreneurs differently. In that way, this study contributes to our understanding of feedback mechanisms as socially situated cognitive phenomena.

\subsection{Limitations AND FUtURE RESEARCH}

This study knows a number of limitations. Firstly, the number of entrepreneurs in our study sample is not large enough to be able to generalize our findings - a constraint often encountered in qualitative research. On a related note, we studied a biased sample of entrepreneurs. Our data was drawn exclusively from diaries of entrepreneurs in a business support environment, limiting it to help-seeking entrepreneurs. Therefore the results cannot be representative, and we are unable to draw general, population-wide conclusions.

Secondly, it might be worthwhile to consider cultural or institutional factors when looking at mentoring effects on entrepreneurial mindset development. Authors have found that these factors matter in opportunity development (Mueller \& Thomas, 2001); however, those factors could not be taken into account in this study. 
A third limitation concerns the form of the data. Diary data is a very 'raw' form of data; the entrepreneurs were free to write down thoughts about their development, only guided by the four 'topics' within each weekly diary. The result is rather chaotic data; sometimes, sentences are not finished, various issues are mentioned in general without going into relevant details or names of the parties involved. However, the resulting real-time data also makes it a very pure and authentic form of research data.

Based on the results of this study, we identify four distinctive research alleys which promise the generation of valuable insights into the process of business opportunity development.

Firstly, the taxonomy of feedback effects developed in this study can help us compare and assess the relative value of targeted feedback. While studies have shown that feedback has a positive effect on opportunity recognition (Ozgen \& Baron, 2007) and cognitive learning (St-Jean \& Audet, 2012), future studies can yield more detailed insights into the way in which various duration periods of coaching affect entrepreneurs differently. This, in turn, could yield more understanding of the effect of long-term intervention during business opportunity development. Research into this area can facilitate the optimization of support systems of business incubation/acceleration, for example geared towards entrepreneurs with differing amount of prior experience or different types of business opportunities.

Secondly, the role of targeted feedback mechanism in the development of different metacognitive abilities can advance our understanding of their development. For example, Haynie et al. (2010a) distinguish between metacognitive awareness, metacognitive resources (in the form of metacognitive knowledge and metacognitive experience), metacognitive strategy) and metacognitive monitoring. Research into the effect of coaching and panel feedback on the development of these metacognitive abilities could yield valuable information about how these abilities are developed by means of different targeted feedback mechanisms.

Thirdly, while qualitative data is increasingly seen as important in business research (Watson, 2011), there is still a world to discover in terms of research methods. More finegrained data collection methods and analyses can provide understanding of unique entrepreneurial processes, whether cognitive or action-based. Modern technology and multimedia offer proficient means for academics to design novel research instruments, for 
example in the form of logbooks, short surveys, or social media applications. For example Foo, Uy \& Baron (2009) advocate a more extensive use and critical evaluation of these methods.

Fourthly, the taxonomy which we developed might find useful application in explaining differences among entrepreneurs in other contexts. For example, this segmentation could help explain ex ante differences in entrepreneurial intentions, but also entrepreneurial goalsetting behavior - such as regarding team development or investment decisions -as well as networking behavior of entrepreneurs outside an institutional support setting. Therefore, further research in this direction is recommended.

Overall, the current study shows that targeted feedback offers useful mechanisms for entrepreneurial cognitive development during business opportunity development, and that the effect of targeted feedback differs depending on the type of entrepreneur. We believe that this study will open the door to further and even deeper study of important mechanisms of feedback in the entrepreneurial ecosystem.

\subsection{BIBLIOGRAPHY}

Bisk, L. (2002). Formal entrepreneurial mentoring: the efficacy of third party managed programs. Career development international, 7(5), 262-270.

Fischer, E., \& Reuber, A. R. (2003). Support for rapid-growth firms: a comparison of the views of founders, government policymakers, and private sector resource providers. Journal of small business management, 41(4), 346-365.

Allen, T. D., Day, R., \& Lentz, E. (2005). "The role of interpersonal comfort in mentoring relationships". Journal of Career Development, 31(3), 155-169.

Baron, R. A. and M. D. Ensley (2006). "Opportunity Recognition as the Detection of Meaningful Patterns: Evidence from Comparisons of Novice and Experienced Entrepreneurs." Management Science 52(9): 1331-1344. 
Baron, R.A. (2014). "Thinking about Cognition and its Central Role in Entrepreneurship: Confessions of a 'Reformed' Behaviorist”. Handbook of Entrepreneurial Cognition, 61-85

Bolger, N., A. Davis, et al. (2003). "Diary Methods: Capturing Life as it is Lived." Annual Review of Psychology 54(1): 579-616.

Cardon, M. S., Wincent, J., Singh, J., \& Drnovsek, M. (2009). The nature and experience of entrepreneurial passion. Academy of management Review, 34(3), 511-532.

Chen, X.-P., Yao, X., and Kotha, S. Entrepreneur Passion And Preparedness In Business Plan Presentations: A Persuasion Analysis Of Venture Capitalists' Funding Decisions. Academy of Management Journal 52, 1 (2009), 199-214.

Clark, C. (2008). The impact of entrepreneurs' oral 'pitch' presentation skills on business angels' initial screening investment decisions. Venture Capital 10, 3 257-279.

Clarke, J. S., \& Cornelissen, J. P. (2014). 11. How language shapes thought: new vistas for entrepreneurship research. Handbook of Entrepreneurial Cognition, 383 - 397

Cohen, D. G. and B. Feld (2011). Do more faster: TechStars lessons to accelerate your startup. Hoboken, N.J., Wiley.

Collewaert, V. and Sapienza, H. J. (2016). How Does Angel Investor-Entrepreneur Conflict Affect Venture Innovation? It Depends. Entrepreneurship Theory and Practice, 40(3): 573597

Cooper, A. C. and W. C. Dunkelberg (1986). Entrepreneurship and paths to business ownership. Strategic Management Journal, 7(1): 53-68.

Cull, J. (2006). "Mentoring young entrepreneurs: what leads to success?". International journal of evidence based coaching and mentoring, 4(2), 8-18.

Corbin, J. M., \& Strauss, A. (1990). Grounded theory research: Procedures, canons, and evaluative criteria. Qualitative sociology, 13(1), 3-21. 
Crompton, B. M., \& Smyrnios, K. X. (2006). Training, Coaching and Mentoring: Supporting High-Technology Entrepreneurs Through Commercialisation and Market Entry Stages. In 51st International Council for Small Business World Conference, Melbourne, Australia., June.

Crompton, B. M., Smyrnios, K. X., \& Bi, R. (2012). Measuring the influence of business coaching on fast-growth firms. Small Enterprise Research, 19(1), 16-31.

Davidsson, P., \& Honig, B. (2003). The role of social and human capital among nascent entrepreneurs. Journal of business venturing, 18(3), 301-331.

Deakins, D., Graham, L., Sullivan, R., \& Whittam, G. (1998). New venture support: an analysis of mentoring support for new and early stage entrepreneurs. Journal of Small Business and Enterprise Development, 5(2), 151-161.

De Carolis, D. M., \& Saparito, P. (2006). Social capital, cognition, and entrepreneurial opportunities: A theoretical framework. Entrepreneurship theory and practice, 30(1), 41-56.

Dew, N., Read, S., Sarasvathy, S. D., \& Wiltbank, R. (2009). Effectual versus predictive logics in entrepreneurial decision-making: Differences between experts and novices. Journal of business venturing, 24(4), 287-309.

Dreyfus, H. L., \& Dreyfus, S. E. (2005). Peripheral vision expertise in real world context. Organization studies, 26(5), 779-792.

Fauchart, E., and M. Gruber. (2011). Darwinians, communitarians, and missionaries: The role of founder identity in entrepreneurship." Academy of Management Journal, 54(5): 935957. 
Filion, L. J. (2004). Operators and visionaries: differences in the entrepreneurial and managerial systems of two types of entrepreneurs. International Journal of Entrepreneurship and Small Business, 1(1): 35-55.

Filley, A. C. and R. J. A. Aldag (1978). Characteristics and Measurement of an Organizational Typology. The Academy of Management Journal, 21(4): 578-591.

Fiske, S.T. \& Taylor, S.E. Social Cognition - From Brains to Culture. 2013. London: Sage Publications, Inc.

Foo, M. D., Uy, M. A., \& Baron, R. A. (2009). How do feelings influence effort? An empirical study of entrepreneurs' affect and venture effort. Journal of Applied Psychology, 94(4), 1086.

Galbraith, C. S., McKinney, B. C., DeNoble, A. F., \& Ehrlich, S. B. (2013). The impact of presentation form, entrepreneurial passion, and perceived preparedness on obtaining grant funding. Journal of Business and Technical Communication, 1050651913513902.

Gerasymenko, V., De Clercq, D., \& Sapienza, H. J. (2015). Changing the business model: Effects of venture capital firms and outside CEOs on portfolio company performance. Strategic Entrepreneurship Journal, 9(1), 79-98.

Grégoire, D. A., P. S. Barr, et al. (2010). Cognitive Processes of Opportunity Recognition: The Role of Structural Alignment. Organization Science, 21(2): 413-431.

Grégoire, D. A., \& Shepherd, D. A. (2012). Technology-market combinations and the identification of entrepreneurial opportunities: An investigation of the opportunity-individual nexus. Academy of Management Journal, 55(4), 753-785.

Gulbranson, C. A., \& Audretsch, D. B. (2008). Proof of concept centers: accelerating the commercialization of university innovation. The Journal of technology transfer, 33(3), 249258.

Haynie, J. M., Shepherd, D. A., \& McMullen, J. S. (2009). An opportunity for me? The role of resources in opportunity evaluation decisions. Journal of Management studies, 46(3), 337-361. 
Haynie, J. M., D. Shepherd, et al. (2010a). A situated metacognitive model of the entrepreneurial mindset. Journal of Business Venturing, 25(2): 217-229.

Haynie, J. M., D. A. Shepherd, et al. (2010b). Cognitive Adaptability and an Entrepreneurial Task: The Role of Metacognitive Ability and Feedback. Entrepreneurship Theory and Practice, 36(2): 237-265.

Haynie, J. M., Shepherd, D. A., \& Patzelt, H. (2012). Cognitive adaptability and an entrepreneurial task: The role of metacognitive ability and feedback. Entrepreneurship Theory and Practice, 36(2), 237-265.

Humphreys, M., Ucbasaran, D., \& Lockett, A. (2012). Sensemaking and sensegiving stories of jazz leadership. Human Relations, 65(1), 41-62.

Jarillo, J. C. (1989). Entrepreneurship and growth: the strategic use of external resources. Journal of Business Venturing, 4(2): 133-147.

Kendall, J. (1999). Axial Coding and the Grounded Theory Controversy. Western Journal of Nursing Research, 21(6): 743-757.

Kor, Y. Y., J. T. Mahoney, et al. (2007). Resources, Capabilities and Entrepreneurial Perceptions*. Journal of Management Studies, 44(7): 1187-1212.

Kraiger, K., Ford, J. K., \& Salas, E. (1993). Application of cognitive, skill-based, and affective theories of learning outcomes to new methods of training evaluation. Journal of applied psychology, 78(2), 311.

Krueger, N. F. (2007). What Lies Beneath? The Experiential Essence of Entrepreneurial Thinking. Entrepreneurship Theory and Practice, 31(1): 123-138.

Krueger, N. (2009). Entrepreneurial Intentions are Dead: Long Live Entrepreneurial Intentions. Understanding the Entrepreneurial Mind. A. L. Carsrud and M. Brännback, Springer New York. 24: 51-72. 
Krueger Jr, N. F. and M. Day (2010). Looking Forward, Looking Backward: From Entrepreneurial Cognition to Neuroentrepreneurship. Handbook of Entrepreneurship Research. Z. J. Acs and D. B. Audretsch, Springer New York. 5: 321-357.

Kutzhanova, N., Lyons, T. S., \& Lichtenstein, G. A. (2009). Skill-based development of entrepreneurs and the role of personal and peer group coaching in enterprise development. Economic Development Quarterly, 23(3), 193-210.

Lafuente, A. and V. Salas (1989). Types of entrepreneurs and firms: The case of new Spanish firms. Strategic Management Journal, 10(1): 17-30.

Lorraine, J., and L. Dussault. (1987). Management behaviors and types of entrepreneurs: The case of manufacturing businesses in the survival and establishment stage. Proceedings of the 32nd World Conference, International Council for Small Business Eds RG Wyckham, LN Meredith, GR Bushe (Simon Fraser University, Burnaby, British Colombia).

Markman, G. D., D. B. Balkin, et al. (2002). Inventors and New Venture Formation: the Effects of General Self-Efficacy and Regretful Thinking. Entrepreneurship Theory and Practice, 27(2): 149-165.

Miron-Shatz, T., Shatz, I., Becker, S., Patel, J., \& Eysenbach, G. (2014). Promoting business and entrepreneurial awareness in health care professionals: Lessons from venture capital panels at medicine 2.0 conferences. Journal of medical Internet research, 16(8), e184.

Mitchell, R. K., Busenitz, L. W., Bird, B., Marie Gaglio, C., McMullen, J. S., Morse, E. A., \& Smith, J. B. (2007). The central question in entrepreneurial cognition research 2007. Entrepreneurship Theory and Practice, 31(1), 1-27.

Mitchell, R. K., Randolph-Singh, B. Mitchel, J. R., 2011. Socially situated cognition Imagining new opportunities for entrepreneurship research. Academy of Management Review, 36(4): 774-776 
Mitchell, R. K., Mitchell, J. R., Zachary, M. A., \& Ryan, M. R. (2014). 13. Simulating socially situated cognition in exchange creation. Handbook of Entrepreneurial Cognition, 412.

Mueller, S. L. and A. S. Thomas (2001). Culture and entrepreneurial potential: A nine country study of locus of control and innovativeness. Journal of Business Venturing, 16(1): 51-75.

Narayanan, V. K., L. J. Zane, et al. (2011). The Cognitive Perspective in Strategy: An Integrative Review. Journal of Management, 37(1): 305-351.

Navis, C., \& Glynn, M. A. (2010). How new market categories emerge: Temporal dynamics of legitimacy, identity, and entrepreneurship in satellite radio, 1990-2005. Administrative Science Quarterly, 55(3), 439-471.

Ozgen, E. and R. A. Baron (2007). Social sources of information in opportunity recognition: Effects of mentors, industry networks, and professional forums. Journal of Business Venturing, 22(2): 174-192.

Pollack, J.M., Rutherford, M.W., and Nagy, B.G. (2012). Preparedness and Cognitive Legitimacy as Antecedents of New Venture Funding in Televised Business Pitches. Entrepreneurship Theory and Practice 36, 5 (2012), 915- 939.

Passmore, J. (2007). An integrative model for executive coaching. Consulting Psychology Journal: Practice and Research, 59(1), 68.

Radu Lefebvre, M., \& Redien-Collot, R. (2013). How to do things with words: The discursive dimension of experiential learning in entrepreneurial mentoring dyads. Journal of Small Business Management, 51(3), 370-393.

Rice, M. P. (2002). Co-production of business assistance in business incubators: an exploratory study. Journal of Business Venturing, 17(2): 163-187.

Saadaoui, S., \& Affess, H. (2015). Evaluating the role of coaching on coaching on developing entrepreneurial self-efficacy. European Journal of Business and Social Sciences, 3(11), 54-61. 
Scillitoe, J. L. and A. K. Chakrabarti (2010). The role of incubator interactions in assisting new ventures. Technovation, 30(3): 155-167.

Schraw, G., \& Dennison, R. S. (1994). Assessing metacognitive awareness. Contemporary educational psychology, 19(4), 460-475.

Seale, C. 1998. Researching Society and Culture. London: Sage Publications Ltd.

Shane, S. and S. Venkataraman (2000). The Promise of Entrepreneurship as a Field of Research. The Academy of Management Review, 25(1): 217-226.

Shepherd, D. A. and N. F. Krueger (2002). An Intentions-Based Model of Entrepreneurial Teams' Social Cognition. Entrepreneurship Theory and Practice, 27(2): 167-185.

Smith, N. R. (1967). The entrepreneur and his firm: The relationship between type of man and type of company. Occasional Papers, Bureau of Business and Economic Research, Michigan State University, 109.

Smith, N. R., and John B. Miner. (1983). Type of entrepreneur, type of firm, and managerial motivation: Implications for organizational life cycle theory. Strategic Management Journal, 4(4): 325-340.

Son, S. J., \& Kim, D. Y. (2014, January). The Effect of Feedback Sources Learning-Goal Orientation on Employee Creativity. In Academy of Management Proceedings (Vol. 2014, No. 1, p. 15502). Academy of Management.

St-Jean, E., \& Audet, J. (2012). The role of mentoring in the learning development of the novice entrepreneur. International Entrepreneurship and Management Journal, 8(1), 119140.

Strauss, A., \& Corbin, J. (1994). Grounded theory methodology. Handbook of qualitative research, 273-285. 
Strike, V., \& Rerup, C. (2016). Mediated sensemaking. Academy of Management Journal, 59(3), 880-905.

Vaughan, D. (1992). Theory elaboration: The heuristics of case analysis. What is a case, 173-202.

Waters, L., McCabe, M., Kiellerup, D. \& Kiellerup, S. (2002). The role of formal mentoring on business success and self-esteem in participants of a new business start-up program, Journal of Business and Psychology, 17(1), 107-121.

Watson, T. J. (2011). Ethnography, Reality, and Truth: The Vital Need for Studies of 'How Things Work' in Organizations and Management. Journal of Management Studies, 48(1): 202-217.

Weick, K. E. (1995). Sensemaking in organizations (Vol. 3). Sage.

Weick, K. E., Sutcliffe, K. M., \& Obstfeld, D. (2005). Organizing and the process of sensemaking. Organization science, 16(4), 409-421.

van Werven, R., Bouwmeester, O., \& Cornelissen, J. P. (2015). The power of arguments: How entrepreneurs convince stakeholders of the legitimate distinctiveness of their ventures. Journal of Business Venturing, 30(4), 616-631.

Wheeler, L. and H. T. Reis (1991). Self-Recording of Everyday Life Events: Origins, Types, and Uses. Journal of Personality, 59(3): 339-354.

Wikholm, J., Henningson, T. \& Hultman, C. M. (2005). Demand of mentoring among new starters. ICSB 50th World Conference, Washington, DC

Zimmerman, M. A., \& Zeitz, G. J. (2002). Beyond survival: Achieving new venture growth by building legitimacy. Academy of Management Review, 27(3), 414-43. 


\section{ChAPTER 3}

\section{Sensebreaking and the DeVelopment of Entrepreneurial Cognition}

Gabi A. Kaffka, University of Twente

I. Singaram, Rollins College, FI (USA)

Jeroen Kraaijenbrink, University of Twente

Aard J. Groen, University of Twente

History of the manuscript: This chapter is a modified version of a peer-reviewed paper that was presented at the RENT 2012 conference in Lyon (France). 


\section{ABSTRACT}

While sensemaking describes the process by which actors fill in a meaning void, sensebreaking is seen as the process of creating that void by disrupting current understandings. We apply the concept of sensebreaking to the analysis of the influence of relevant stakeholders on entrepreneurial cognition. Specifically, we study how those stakeholders affect sensemaking processes during opportunity identification and preparation. Using a real-time, longitudinal data set of weekly diary entries we followed 30 entrepreneurs engaged in new venture building throughout one year. The analysis of almost 7,200 distinct units of analysis revealed 115 occurrences of sensebreaking. These show that sensebreaking aids the development of entrepreneurial cognition via three possible actions: questioning, reframing and redirecting. We identify relevant stakeholders involved in sensebreaking and discuss the typical effects of the most important stakeholders in sensebreaking and new sensemaking action. In this way we contribute to the conceptualization of micro-level foundations of entrepreneurial (social) cognition.

\subsection{INTRODUCTION}

Entrepreneurial cognition refers to knowledge structures used for opportunity identification, preparation and exploitation. These knowledge structures are associated with mental model or prototype building, pattern recognition and decision-making (Baron \& Ensley, 2006; McMullen \& Shepherd, 2006; McKelvie et al., 2009). Central to the study of entrepreneurial cognition are sensemaking processes (Michell et al.; 2011). Sensemaking is associated with the ex ante or ex post construction of meaning about a novel or unexpected process, event or situation (Weick et al., 2005; Weick, 2012).

Right from connecting the dots to identify a new opportunity (Baron \& Ensley, 2006) or the use of emergent entrepreneurial strategies in place of methodical planning (Sarasvathy, 2001) to devising a strategy as a set heuristics (Bingham \& Eisenhardt, 2011), entrepreneurs are engaged in sensemaking processes. Entrepreneurial sensemaking serves as the source of development of entrepreneurial cognition and, ultimately, business opportunity identification and preparation. However, despite the importance of sensemaking processes in organizational contexts (Weick et al., 2005) it is a neglected topic in the field 
of entrepreneurship. Empirical studies on the micro-level development of those cognitions are sparse (Krueger, 2003; 2007). Also, we lack understanding of the antecedents of entrepreneurial sensemaking (Shepherd et al, 2014).

Intersubjective sensemaking is fundamental to entrepreneurial opportunity development. The field of entrepreneurship has recognized the socially situated nature of cognitive processes and the effect of input of various stakeholders on them (Mitchel et al., 2011). Entrepreneurship literature recognizes the role of intersubjectivity in entrepreneurship (Davidsson, 2001; Venkataraman et al., 2012). Studies have analyzed the role of team members, mentors and other actors for entrepreneurial cognitive development (West, 2007; Ozgen \& Baron, 2007). Various studies have shown that stakeholders positively affect identification and preparation of a business opportunity in the form of information, feedback and advice (Ozgen \& Baron, 2007; Sarasvathy, 2008).

However, studies have neglected to analyze the effect of intersubjective sensebreaking on entrepreneurial cognition. Sensebreaking discrupts a person's existing understanding or process of sensemaking by contradictory evidence. Sensebreaking is indispensable for new or different sensemaking processes (Pratt, 2000).

Intersubjective sensebreaking occurs through interaction with stakeholders such as investors or customers. Acts of sensebreaking by third parties affect sensemaking processes, by challenging existing assumptions and thus creating a meaning void (Pratt, 2000; Vlaar et al., 2008). They make entrepreneurs realize logical holes in their arguments, broaden their perspective and trigger a new or different focus throughout the opportunity identification and preparation phase. In doing so, sensebreaking occurrences facilitate the adding of new or different 'dots' when they are 'connecting the dots' (Baron \& Ensley, 2006) for the realization of a viable business opportunity. Entrepreneurial sensemaking processes affect entrepreneurial cognition and ultimately opportunity identification and preparation. Intersubjective sensebreaking can therefore play an important role during business opportunity development.

In this study, we examine the effects of intersubjective sensebreaking on the development of entrepreneurial cognition and which stakeholders contribute to this. We use 
qualitative data from weekly diary entries provided by entrepreneurs over a year. Applying discourse data analysis we find that sensebreaking acts involving a range of stakeholders. The results show that sensebreaking enables novel sensemaking processes of entrepreneurs vis-à-vis a variety of topics relating to opportunity development. In that way, sensebreaking functions as mechanism for entrepreneurial cognitive development.

This study contributes in the following ways. For one, the results contribute not only on empirical insights but also to current theory building efforts on the development of metacognition and socially situated cognition within entrepreneurship studies. Specifically we add to the understanding of contributions made by pertinent stakeholders to entrepreneurial cognitive processes. In that way we inductively conceptualize the role which sensebreaking plays in entrepreneurship. Secondly, our data collection method via diary entries enable us to observe and report on the origins of entrepreneurial cognition with very limited retrospection bias. This allows us to perform a micro-level analysis of interactions between entrepreneurs and specific stakeholders over time and their real-time cognitive effects. Finally, this study illustrates the beneficial effects of sensebreaking on cognitive development: The different elements or mechanisms of sensebreaking could therefore be particularly useful for feedback sessions in incubation/acceleration environments.

\subsection{THEORY}

Entrepreneurial cognition consists of knowledge structures which are used in interpretive and decision-making processes during business opportunity identification, preparation and exploitation (Mitchell et al., 2002; Grégoire et al., 2011). Research on entrepreneurial cognition has found that entrepreneurs use previous knowledge to 'connect the dots' and leverage mental models, metaphors and symbols in order to identify and successfully prepare a business opportunity (Hill \& Levenhagen, 1995; Baron \& Ensley, 2006; Zott \& Huy, 2007; Cornelissen et al, 2012). Cognitive differences at the individual level may determine how actors would execute entrepreneurial tasks (Forbes, 2005).

The role of supporting stakeholders in the development of a business opportunity such as founding team members, early investors, mentors, launch customers and sometimes 
close family members and friends has been empirically proven (Ozgen \& Baron, 2007; Lim et al., 2013). In terms of the actors that shape cognition, attention has been paid to particular stakeholders such as the top management team (West, 2007) and groups consisting of founders and investors (Lim et al., 2013). Ozgen and Baron (2007) describe the role of mentors and other industry-related network actors in shaping entrepreneurial cognition. StJean \& Audet (2012) show that these parties offer different types of support in addition to industry knowledge such as emotional support and support for cognitive development. And Gemmell, Boland and Kolb (2012) found that shared cognition between entrepreneurs and trusted partners can help them generate creatively rich ideas.

However even after having embraced the often quoted notion that the "entrepreneur in the entrepreneurship is probably plural" (Gartner et al., 1994), we know very little about the effect of micro-level interactions between the entrepreneurs and each of these stakeholders on cognitive development of entrepreneurs. Micro-level empirical evidence of how stakeholders affect entrepreneurial interpretative and decision-making processes is still lacking.

\section{SENSEMAKING AS A SOCIALLY SITUATED COGNITIVE PHENOMENON}

The role of entrepreneurial cognition in the intersubjective construction of opportunities is conceptualized by Mitchell et al. (2011) as socially situated. Socially situated cognition (SSC) sees cognition as action-oriented, embodied, situated and distributed. Sensemaking plays a central role in socially situated cognition (Mitchell et al., 2011). In this perspective, sensemaking of entrepreneurial opportunities is seen as the result of intersubjective knowledge collaboration between stakeholders involved (Davidson, 2001; Mitchell et al., 2011; Venkataraman et al., 2012). Various authors have recognized that "cognition is grounded in the sensemaking" of actors (Thompson, 2007; Thompson \& Stapleton. 2009).

In this study, we adopt a socially situated perspective on sensemaking. Using this perspective on entrepreneurial cognition, we examine the way in which entrepreneurs' sensemaking is affected by the input from stakeholders during opportunity preparation.

Sensemaking is the arrangement of information into meaningful patterns (Weick et al., 2005) in order to process, store, or apply new or existing information. Sensemaking has 
been widely acknowledged as an important field of organization studies (Weick et al., 2005), as well as in the field of entrepreneurship studies. For example, research found that entrepreneurs identify new business opportunities by organizing prior knowledge with new insights; this process has been described as 'connecting the dots' (Baron, 2004; Baron \& Ensley, 2006). Sensemaking organizes the 'flux' of the dynamic reality we are part of, by noticing, bracketing labeling information that is considered relevant or useful (Weick, 1995). In the case of entrepreneurship, Bettiol et al. (2011) find that sensemaking facilitates market activities of entrepreneurial ventures. While sensemaking describes how the environment works, the related concept of sensegiving is associated with communicating that representation of reality to others in order to gain their support (Hill \& Levenhagen, 1995).

Entrepreneurs engage in sensemaking and sensegiving processes for example by metaphor development to communicate their vision to external partners, which is considered an important aspect of entrepreneurial activities (Hill \& Levenhagen, 1995). Concepts of sensemaking and sensegiving are both associated with an individual's reaction to 'broken down' logics in a social context and with ways of fixing those breakdowns. In the past, studies have mainly focused on sensemaking and sense-giving processes by entrepreneurs (Hitt \& Levenhagen, 1995; Zott \& Huy, 2007; Brännback \& Carsrud, 2009; Holt \& Macpherson, 2010; Cornelissen et al., 2012).

However, the concepts of sensemaking and sensegiving do not offer explanations as to how that 'breaking down' occurs initially. More recently, the term 'sensebreaking' has emerged in organizational studies (Pratt, 2000; Vlaar et al., 2008) which helps us to identify the socially situated antecedents of entrepreneurial cognition.

\section{SensebreAkING}

Sensebreaking is an integral part of the sensemaking process. It is concerned with breaks in the scanning, interpretation and learning dynamics of the sensemaking process (Vlaar et al., 2008). Sensebreaking occurs when a person's existing understanding or process of sensemaking is disrupted by contradictory evidence or values (Vlaar et al., 2008; Pratt, 2000). In that way, sensebreaking creates a meaning void, enabling subsequent 
sensemaking to take place. The concept of sensebreaking therefore contributes significantly to new sensemaking processes and ultimately the development of entrepreneurial cognition.

In organizational contexts, prior studies have analysed the consequences of sensebreaking that ensue as a result of failure of strategic change (Lawrence \& Maitlis, 2005; Mantere et al., 2009; Maclntosh \& Beech, 2010). A different approach was taken by Pratt (2000) who used the concept of sensebreaking for the construction of self-identity. And Vlaar et al. (2008) explain the usefulness of sensebreaking episodes from studying geographically distributed team members training one another. They find that sensebreaking triggered by others help team members to question their existing assumptions and learn from it. In the case of entrepreneurship sensebreaking occurs when an entrepreneur's sensemaking activity is interrupted by the introduction of new evidence that challenges their current assumptions.

Sensebreaking occurs by critical self-reflection but it can also be intersubjectively triggered by stakeholders. This makes intersubjective sensebreaking an interesting analytical tool for the study of third party influence on business opportunity development. Sensebreaking instances induce entrepreneurs to reframe their interpretations of a situation, redirect their strategy or actions, or question the entrepreneur's learning and current understanding (Vlaar et al., 2008). These mechanisms of questioning, reframing and redirecting are central elements to our study of sensebreaking. Therefore we describe each one in more detail.

Reframing involves the changing of beliefs and attitudes in the process of making sense of new information (Spillane et al., 2002). It is associated with the unsettling or replacement of existing understandings, previously held conceptions, and lines of thinking by others (Vlaar et al., 2008). Though reframing requires something that is 'already known' it involves a deep conceptual reorganization of explanatory reasoning in order to accounfor discrepant or deviant cases or situations encountered (Spillane et al., 2002).

Redirecting implies a notion of something existing which will be transformed; it thus requires an existing behavior or idea which will be 'worked upon'. For example, it can relate to the replacement of one object of attention, such as one problem as center of attention with another one (Van Merriënboer et al., 2002). Another usage of redirecting is within 
teams, where it can be used to draw other team members' attention to other aspects and search for different solutions for a given problem (Vlaar et al., 2008). Redirecting requires that there is a given extent or degree of attention already present - as in the case of the problem, initial attention for the problem - that is veered towards something else, such as a different problem. An original belief or attitude regarding a problem, however, remains unaltered. For example, Van Merriënboer et al. (2002) suggest, on skills, that redirecting attention is a promising approach to improve training efficiency.

Questioning is meant to interrupt undesirable courses of action actors have taken or are taking (Lawrence \& Maitlis, 2007; Vlaar et al., 2008). Questioning is "motivated by the need to problematize the understandings held by others" (Vlaar et al, 2008: p. 241) so that they "question the bases on which they have been acting" (Lawrence \& Maitlis, 2007: p. 15). Borchert \& Rocheford (2009) showed that negative feedback during opportunity exploitation such as questioning positively affects the extent of product change but not the innovativeness of the product.

In this study, we extend the notion of sensebreaking to the entrepreneurial context and conceptualize sensebreaking as the effect of stakeholders on cognitive development of entrepreneurs engaged in nascent stages of venture building, commonly described as the phase of opportunity recognition (Baron \& Ensley, 2006), discovery (Davisson \& Honig, 2003) or identification (Grégoire \& Shepherd; Shepherd et al., 2014;).

Sensebreaking is seen as influencing the development of entrepreneurial cognition during venture development by yielding possibilities for the creation of new knowledge structures which are analysed in terms of how stakeholders redirect, reframe and question the entrepreneur's opportunity development.

\section{EFFECT OF SENSEBREAKING ON ENTREPRENEURIAL COGNITION: NOVEL SENSEMAKING OF RESOURCES}

Sensebreaking by third parties creates a void of meaning that, in turn, yields opportunities for new assessments and interpretations. Those assessments and interpretations are associated with entrepreneurial sensemaking. Entrepreneurial sensemaking pertains to individual cognitive structures reflecting decision-making processes about the control and allocation of firm resources (Foss et al., 2008). Previous 
studies have shown that third parties play an important role in entrepreneurial sensemaking (McMullen, 2013).

Opportunity identification and preparation require (im)material resource acquisition and orchestration crucial for successful business development (Jarillo, 1989; Barney, 1991; 2011; Davidsson \& Honig, 2003; Sirmon et al., 2010) however is generally acknowledged that resources are not inherently valuable (Kor et al., 2007; Kraaijenbrink et al., 2010). In this study, we analyze the effect of sensebreaking on the development of entrepreneurial cognition by examining entrepreneurs' sensemaking about resources in business opportunity development. By questioning existing assumptions, redirecting the entrepreneur's attention and reframing their held assumptions and beliefs, stakeholders affect entrepreneurial sensemaking about the value, acquisition and orchestration of (im)material rescoures.

On the basis of existing literature, we distinguish three broad categories of resources that are of particular relevance for opportunity development. These are economic capital, human capital and social capital. Economic capital pertains to material resources such as financial capital or material possessions such as housing, office supplies, machines or technologies. Economic capital also includes intellectual capital due to the fact that it can be expressed in terms of monetary value (Nahapiet \& Ghoshal, 1998). Human capital represents the knowledge, skills and capabilities of individual actors (Coleman, 1988; Hitt \& Douane, 2002), acquired for example by education, professional experience and prior entrepreneurial experience (Hitt \& Duane, 2002; Davidsson \& Honig, 2003; Florin et al., 2003; Haynie et al, 2009). Social capital in the form of networks of relationships provide actors with 'a 'credential' which entitles them to credit, in the various senses of the word' (Bourdieu, 1986: p. 249). In the case of entrepreneurship, social capital matter in the form of providing legitimacy (in the form of credible and competent contacts) and linkages to potential investors, lead customers or other social sources of tangible and intangible support (Davidsson \& Honig, 2003; Ozgen \& Baron, 2007). The relevance of economic, social and human capital has been confirmed in various empirical studies (Davidsson \& Honig, 2003). 
In this study, we examine changes in the way that entrepreneurs asses the value of resources as a result of intersubjective sensebreaking in terms of human, social and economic capital in order to successfully develop their opportunity.

\subsection{METHOD}

\section{RESEARCH DESIGN}

In this study, we examine how entrepreneurial cognition develops via meaningful coconstruction of reality by actors involved in business opportunity development. To do so, we use a qualitative research approach. We do so in order to collect data on the subjective reports of respondents regarding the way other parties use sensebreaking, and the effect of this on their own sensemaking processes. Hindle (2004) cites three major hurdles in the choice of qualitative methods to study entrepreneurial cognition, namely introspection, holism and temporality. In order to combat these methodological issues we make use of the diary method as measuring instrument in the study of entrepreneurial cognition.

\section{DATA COLLECTION INSTRUMENT}

We measured the effect of stakeholders on the development of entrepreneurial cognition by examining written self-reports by entrepreneurs. These self-reports are digital diaries which consists of a diary for weekly entries.

Diary studies have a long tradition in the field of psychology because they offer the opportunity to investigate micro-level processes within their natural context. They are suitable for three types of data collection goals namely, 1) reliable person-level information, 2 ) estimates of within-person change over time as well as individual differences in that change and 3) the causal analysis of within-person change and differences in that change (Bolger et al., 2003). Since we are interested in the development of entrepreneurial cognition at the individual level and the simultaneous progress of the new venture, diary analysis fits very well with the purpose of our study. A particular strength of diary research is the high validity and reliability of the qualitative data collected through nearly real-time accounts of individual experience with reduction in biases due to retrospection. Also, the diary method 
is suited for longitudinal data collection, therefore this method can be used to deal with the issue of temporality in qualitative research.

The psychology literature distinguishes between three types of diary collection methods; interval, signal, and event contingent diary design (Wheeler and Reis, 1991; Bolger et al., 2003). The interval-contingent protocol requires participants to communicate their experiences at regular, predetermined intervals. Both the signal-contingent and the event-contingent protocol design prompt participants to self-report each time the given signal or event occurs.

For this study we used the interval-contingent protocol. The main reason for this is that due to the 'heat of the moment' of entrepreneurial action, entrepreneurs may not be reflexively aware of which events or signals deserve reporting. Furthermore, the use of regular intervals, allowed us to make the habit of filling out the diaries an integral part of the program. The time-based design of this study involved weekly intervals for the diary collection. The frequency of weekly intervals was judged as appropriate since it allows sufficient time for entrepreneurs to deploy their regular activities. At the same time, they could report on the progress of their ventures with as little retrospection as possible.

We designed the diary entries to capture a limited number of topics. Every diary entry consisted of themes - we call them 'subentries'- which the entrepreneurs could freely elaborate on. There were no word limits. The diary entries were subject to some revisions in a first pilot phase. This pilot showed the need for some more adjustments. The answers given to the first two questions turned out to be too general and did not provide sufficient detail, particularly about the cognitive aspects we were interested in.

Based on the feedback received on an initial diary pilot, the final diary was restructured along the following four sub-entries: 1) Learning: What were the most important things that you learned in the past week? (2) Results: What results have you made in the past week? 3) Issues: What issues have you been most concerned with in the past week? and 4) Next Steps: What are the next steps that you are going to take in the coming weeks? Our analysis of a second batch of diaries showed that these questions did provide the kind of detailed data about the entrepreneur and the venture that was needed. 
The qualitative research method chosen yields useful data for the study of cognitive development by entrepreneurs. In addition, we retrieved the quantifiable personal attributes of entrepreneurs - prior start-up experience - relevant for this study from a survey that is conducted among all respondents at the time of exiting the one-year business incubation/acceleration program.

\section{SAMPLE SELECTION}

The study took place in an institutionalized business incubation/acceleration setting. Our sample consists of entrepreneurs who participated in a one-year business acceleration program in the Netherlands. During this year entrepreneurs interact with various parties within the program: mentors/business coaches, trainers, subject matter experts, an expert panel, fellow participants etc. In addition, entrepreneurs also interact with parties outside the program, such as their friends and family, potential customers, investors, certification agencies and others. For our analyses we used detailed micro-level data from diaries that entrepreneurs filled in during the entire year as part of the accelerator program. The fillingin of the diary was a mandatory requirement agreed explicitly between the accelerator and the entrepreneur.

We selected dairies which contained on average 30 weekly entries at least, and of which the entries were distributed across the year of the incubation/acceleration program. This was done in order to control for potential variations in the incubation/acceleration program which could lead to differential access or availability of stakeholder interactions offered within the realm of the incubation/acceleration program. In that way we sought to ensure a valid and representative picture of sensebreaking occurrences across our sample population. On average, respondents reported in their logbooks 35 weeks during the year.

We sought to examine the effect of sensebreaking on entrepreneurs during business opportunity identification and preparation. Therefore we only sampled respondents who are engaged in novel, independent opportunity identification and preparation. In order to control for the effect of prior start-up experience, in addition to novice entrepreneurs we sampled entrepreneurs with more than six years of entrepreneurial experience. 
Various studies have pointed out that increasing entrepreneurial experience affects self-employment success as well as entrepreneurial cognition (Robinson \& Sexton, 1994; Bingham \& Eisenhardt, 2011). Therefore we sought to have variation in our population with regards to prior start-up experience. By doing so we seek to control the results of our analysis for the moderating role of this attribute in the effects of sensebreaking on entrepreneurial cognition.

While formal education and gender might play a role in entrepreneurial cognition as well (Baron \& Ensley, 2006), we chose not to sample our study population for these attributes. That is due to the dominant presence of highly educated male entrepreneurs in the business accelerator program. The business incubation/acceleration program VLT Twente is coordinated by the technical university (University of Twente) and located in the proximity of it. We observed that many aspiring - but also experienced entrepreneurs - were engaged in business opportunity development in technical fields, such as software and IT services, or machinery, equipment and components. Gender as well as education of entrepreneurs in the incubation are therefore extremely biased among the studied population and we are unable to have a sufficient variety in respondents to control for the effect of these variables.

Our research sample consists of 30 entrepreneurs who are involved in opportunity identification and preparation. We derived this sample from an overall population of 50 entrepreneurs who had completed the VLT incubation/acceleration program and who had filled in a sufficient amount of diary entries throughout that year: at least twenty weekly entries. That means they had started gestation activities for their venture no earlier than two years prior to the start of the program and are still involved in developing that business opportunity. All had finished their one-year business acceleration program at the time the data collection for this study took place. Of the 30 entrepreneurs, only four are female. At the time of data collection for this study, the entrepreneurs in our sample have an average age of 45 years.

As one would expect, some entrepreneurs were very elaborate and descriptive while some of them were characteristically taciturn. It must be noted that each diary entry could contain multiple issues on several topics and thus amounted to multiple units of analysis. At 
an average of 2 units of analysis per entry, four entries per week, and an average of 30 weekly entries per respondent, the diaries yielded more than 7,200 units of analysis.

\section{Coding Procedure and Data Analysis}

Qualitative data analysis, in particular discourse analysis, is carried out by a datacoding process that involves the development of categories to describe consistencies across cases, also called cross-analysis (Silvermann, 2001; Seale, 1998; Hill et al., 1997). By means of comparing data from various sources and developing a system of meaningful categories, we can distinguish frequently occurring topics and meaningful concepts in the reports of respondents. We applied this process to study the effect of sensebreaking on entrepreneurial cognition. First, all diaries were entered into the qualitative software program NVIVO.10. Then, diary instances deemed relevant by the researchers were coded into sensebreaking acts according to the three mechanisms of reframing, redirecting and questioning.

During the coding and cross-analysis of data, researcher triangulation (Flick, 2007) was employed in order to ensure validity of the data analysis. To facilitate researcher triangulation we designed a codebook on the basis of existing literature of sensebreaking, presented earlier. We operationalized the sensebreaking construct in terms of reframing, redirecting and questioning. The codebook contains signal words provide empirical indicators that facilitate the identification of relevant quotes for this study in the diary reports, and subsequently increases the reliability of the coding process. Signal words can be phrases, expressions or verbal structures for the three sensebreaking acts of reframing, redirecting and questioning.

Three researchers independently read the diaries and retrieved those quotes which according to them reflect sensebreaking instances. The results of a first coding round of 15 respondents were thoroughly discussed among the three coders. Based on in this initial coding round, the codebook for the different sensebreaking mechanisms - redirecting, reframing and questioning - was sharpened. In Table 3-1 the indicators of three sensebreaking mechanisms are exemplified by signal words. The table also contains example quotes for each sensebreaking mechanism so as to demonstrate a typical, empirical example of this mechanism from the diary reports. 


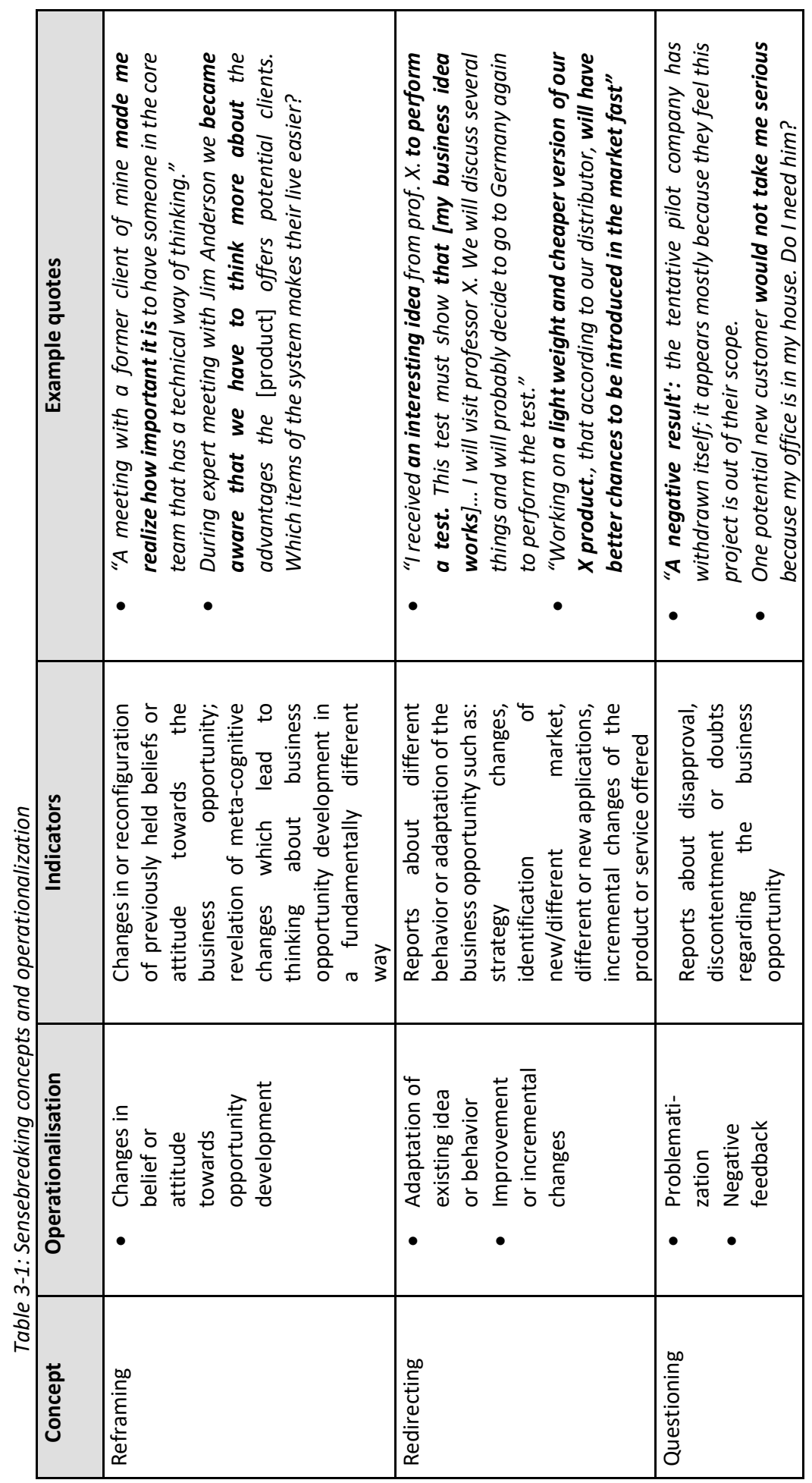


After coding relevant quotes from the diaries into the three categories redirecting, reframing and questioning we categorized the different stakeholders mentioned in those quotes. As indicators for this served the entrepreneurs' naming of actors or external parties. As much as possible, we grouped various parties into the same stakeholder category, for example we categorized banks and business angel investors as 'Financial Investors'. A number of stakeholder categories that we identified represent well-known stakeholders in entrepreneurial opportunity development such as formal networks, suppliers, and (potential) customers. In addition, we also distinguish between different categories of stakeholders in the business incubation/acceleration program such as 'Mentors/Coaches' and 'Trainers' since these appeared to play distinct roles in sensebreaking.

While we included a variety of stakeholders inside and outside the business incubation/acceleration program, we decided to exclude sensebreaking related to insights from books, magazines and other literature since these do not consist of social actors sui generis and are therefore not relevant for the analysis of interactive sensebreaking occurrences.

The analysis yielded 115 instances of sensebreaking. Those instances were used to examine the effect of sensebreaking occurrences on entrepreneurial cognition. We grouped those instances into distinctive, re-occurring themes. For example, several entrepreneurs reported the effect of sensebreaking on one's business plan, therefore we assigned these quotes to the theme of 'strategy'.

\subsection{RESULTS}

Below we present the findings of the analysis. They pertain to differences among the sensebreaking mechanisms of redirecting, reframing and questioning in terms of 1) frequency, 2) categories of stakeholders involved in sensebreaking and 3) effects of those stakeholders on new or different sensemaking processes. 


\section{DIFFERENCES IN FREQUENCY OF SENSEBREAKING DIMENSIONS}

In total, we identified 50 occurrences of redirecting in the diaries, as well as 38 occurrences of reframing. Questioning occurrences were counted the least; we identified only 27 occurrences in the entrepreneurs' diaries. In Graph 1 the total distribution of sensebreaking reports among the three sensebreaking mechanisms is presented in percentage of the total amount of sensebreaking occurrences. This graph shows that $44 \%$ of sensebreaking occurrences reported by the respondents in their diaries pertains to the mechanism of redirecting. This is followed by reframing occurrences which make up $32 \%$ of the reported sensebreaking occurrences reported by the respondents. The other $24 \%$ of the reported sensebreaking occurrences are related to the questioning mechanisms.

Graph 3-1: Distribution of reported sensebreaking mechanisms in percentage of total reported sensebreaking occurrences

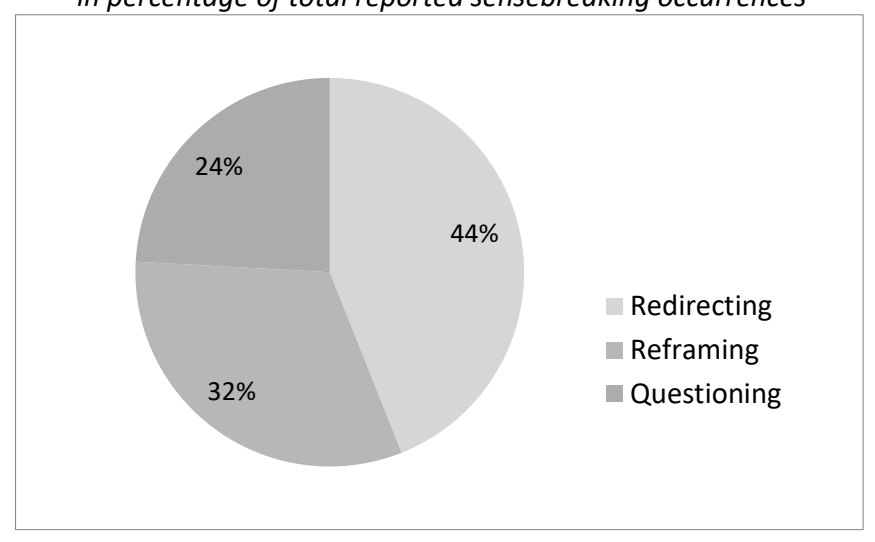

Typically, reframing instances pertain to a change in values emphasized or beliefs held by the respondents. They are related to entrepreneurial opportunity development in terms of basic assumptions of how opportunities are identified and/or prepared. Redirecting occurrences lead to respondents reconsiderations of strategic or tactical decisions, such as which market to enter or how to best create value. Questioning instances are typically related to feedback about the value capture and deliver of the opportunity developed, usually in terms of its finances and product- or service-content. 


\section{DIFFERENCES IN STAKEHOLDERS INVOLVED IN SENSEBREAKING}

Stakeholders involved in sensebreaking that were mentioned by the respondents are assigned to one of ten categories. Five of these categories are associated with the business accelerator program. Those are coaches, trainers, fellow program participants, expert trainers and coaches as well as expert panel members. The category of expert trainers/coaches consists of (inter)national professionals and (academic) experts in a specific business-related field, such as marketing, strategy or opportunity recognition and development. The category of expert panel members contains experienced entrepreneurs or academic faculty members in the field of business administration. At a three-month interval throughout the one-year incubation/acceleration program, the participating entrepreneurs pitched their business plan to an expert panel for feedback.

The other five categories were independently accessible to the entrepreneur stemming from his or her own network, such as the non-formal network of the entrepreneur (friends, families or former colleagues or employers etc.), as well as form al networks which included governmental bodies and various other institutes (universities, research organizations). Furthermore there was a category for financial institutions - including banks, investment funds and business angels, and a category 'team' which consists of the entrepreneur's own employees or management team members. In Graph 3-2 we show the distribution of total sensebreaking instances among the stakeholder categories.

Graph 3-2 Distribution of sensebreaking among stakeholder categories, in percentage of total sensebreaking occurrences

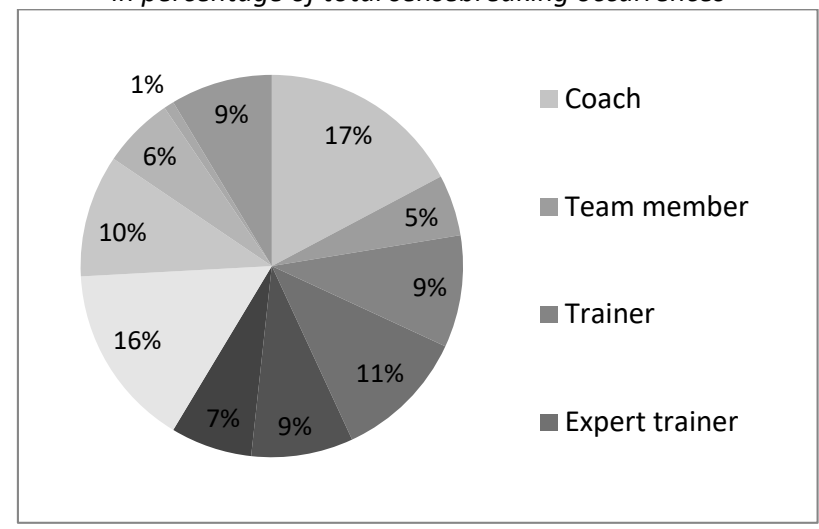


Graph 3-3 contains the reported stakeholder groups involved in sensebreaking (in percentage of the total amount of reports per sensebreaking mechanism). Overall, we see that the most frequently reported stakeholder categories are coaches and (potential) customers. We also see that the various stakeholders differ in the way they trigger the sensebreaking mechanisms of redirecting, reframing and questioning

Reframing occurrences are mainly prompted by coaches and (expert) trainers/coaches of the VLT incubation/acceleration program whereas redirecting episodes are predominantly triggered by interaction with the non-formal network and (potential) customers of the entrepreneurs. The category of (potential) customers also make up the dominant category involved in questioning occurrences.

Graph 3-3 Distribution of sensebreaking per stakeholder group (in \% of total sensebreaking reports of respectively questioning, reframing and redirecting)

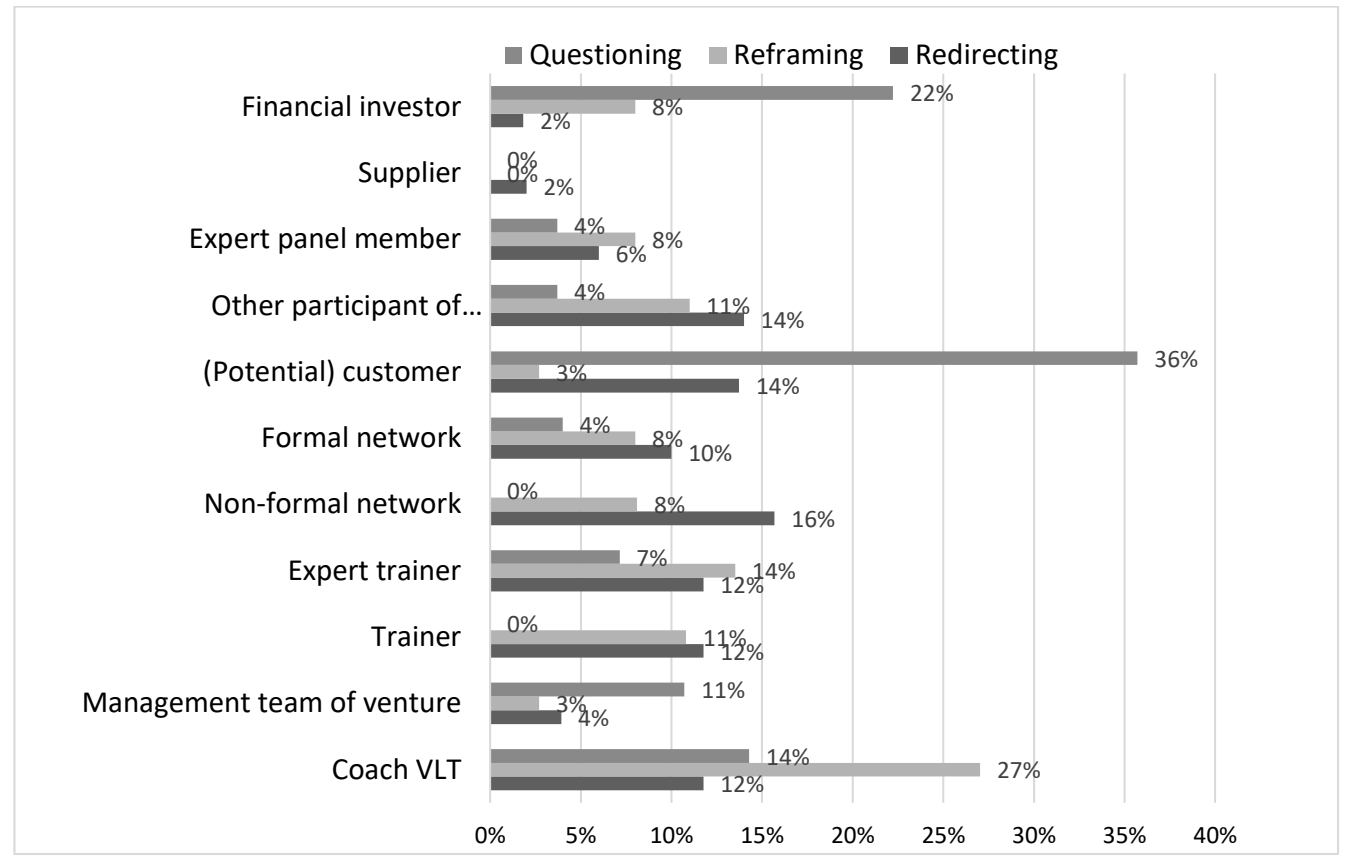

\section{SENSEBREAKING AND ITS EFFECTS ON ENTREPRENEURIAL COGNITION}

We also examined the effects of sensebreaking occurrences on entrepreneurial sensemaking. In Table 3-2 we show the themes involved in those novel sensemaking 
processes. Some sensemaking themes were only triggered by one or two of the three sensebreaking mechanisms, which is reflected in the absence of quotes in the table.

Sensebreaking most often triggers novel sensemaking about human capital resources. The themes of strategic reconsiderations and business planning are mentioned most frequently. Here, novel sensemaking pertains to the applicability of the business opportunity and are mainly prompted by redirecting occurrences. For example, respondents report being redirected to other methods of business opportunity development, such as different commercialization of the envisioned product or service or the entry to other or new markets. There are also some reports of reframing instances; respondents mention that they realize they fundamentally have to change their thinking about business planning or market approaches. We note that questioning plays hardly a role in the reports of these themes; only once is questioning the trigger for novel sensemaking in these two overall most often reported themes.

Other dominant themes in novel sensemaking of human capital relate to the opportunity's value proposition, the business model and the development of one's customer orientation. All three of are frequently recurrent themes in both redirecting and reframing sensebreaking instances. Central to this dimension is the entrepreneur's concern of turning the product or service which is developed into a value that responds effectively to those problems. Novel sensemaking processes related to these themes are sometimes triggered by questioning occurrences as well; we identified reports of quite some negative feedback on collaboration with specific parties, and refusals by potential customers, as well as difficulties in negotiations, such as form and content of sales and orders.

Less frequently mentioned are themes associated with personal and skill development. For example, we identified novel sensemaking about one's own role in the venture but also about home-work balance. Entrepreneurs report both redirecting and reframing but there are considerably more reframing occurrences associated with personal (skill) development. Yet other themes regard changes in organizational structures of the business opportunity. This pertains to the statutory organizational form, the design of official rules and the set-up of legal business contracts of the venture. Novel sensemaking associated with organizational structures are mainly caused by the sensebreaking mechanism of questioning. 
Redirecting occurrences also led to novel sensemaking of social capital. This concerns predominantly interaction with stakeholders which redirect the entrepreneur's attention to other or new network ties for and for acquisition. For example, entrepreneurs report novel sensemaking regarding importance of key customers, key suppliers as well as finding collaborative partners. These are mainly triggered by interaction with the non-formal network and the entrepreneur's coach.

Sensebreaking occurrences that trigger novel sensemaking of economic capital, such as financial or material aspects, are reported only sporadically by the respondents and result from redirecting and questioning instances. Novel sensemaking associated with the themes of tangible or material resources pertain to the benefits of funding, prototype development or particular equipment needed. Interestingly, the theme of funding is caused by questioning instances which involve declined funding or dissatisfaction with turn-over by banks. 


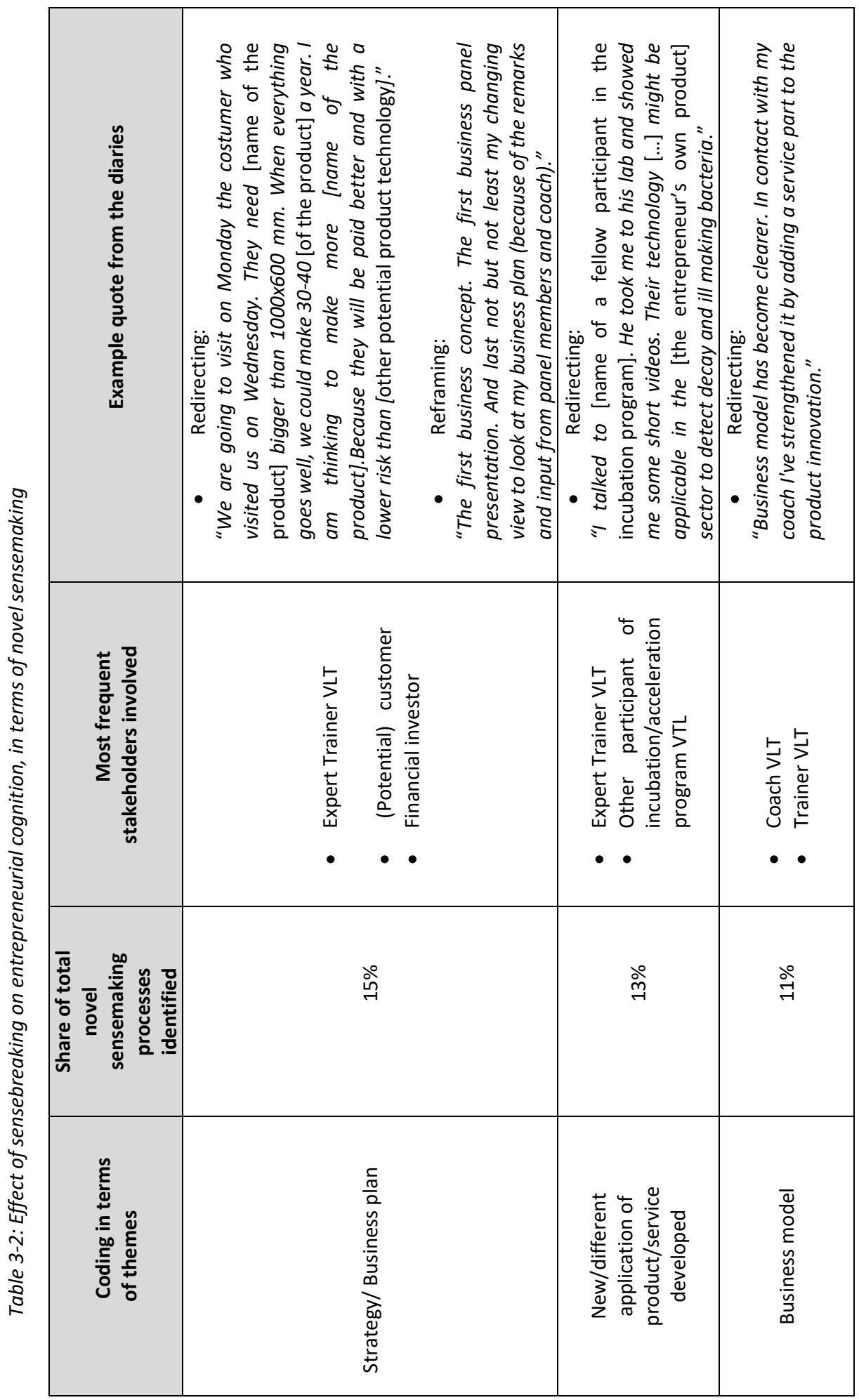




\begin{tabular}{|c|c|c|c|c|c|}
\hline 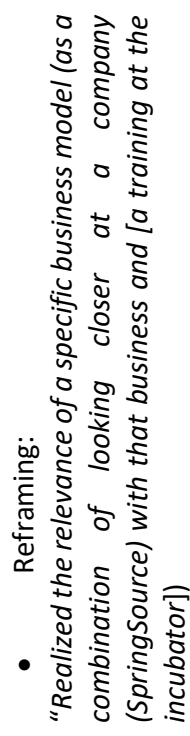 & 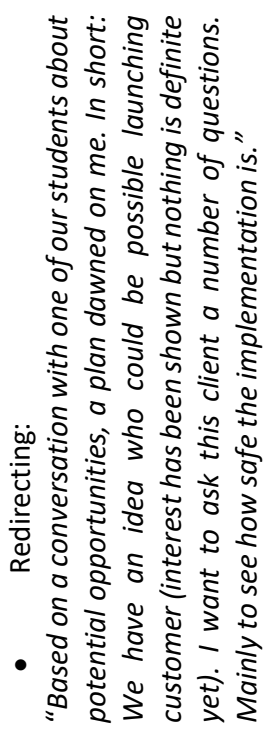 & 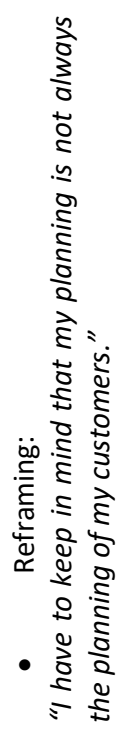 & 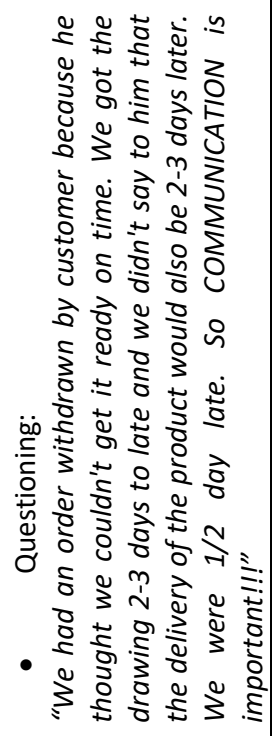 & 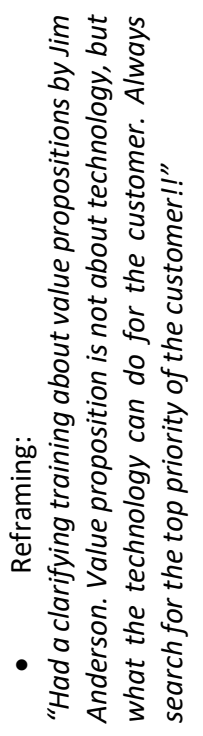 & 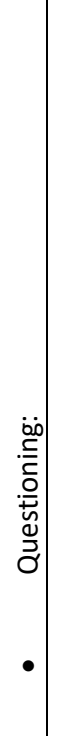 \\
\hline & & 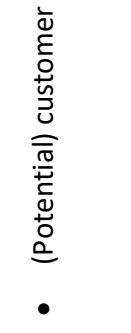 & & 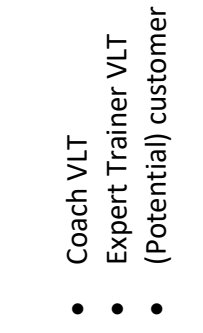 & \\
\hline & & ১े̊ & & ১̊ำ & \\
\hline & & 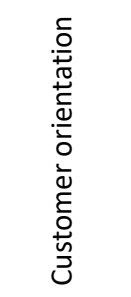 & & 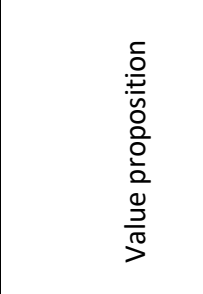 & \\
\hline
\end{tabular}




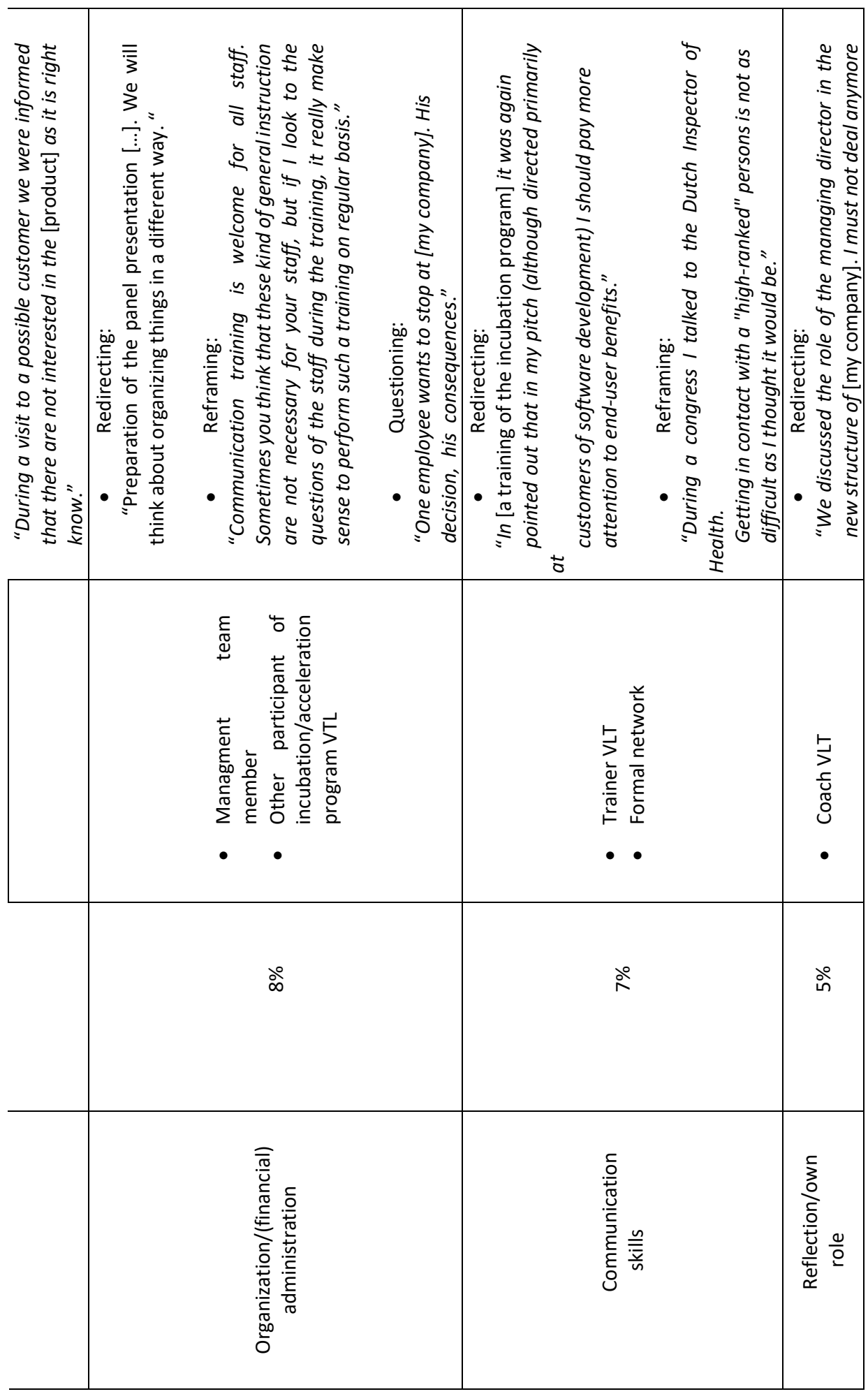




\begin{tabular}{|c|c|c|c|c|c|}
\hline 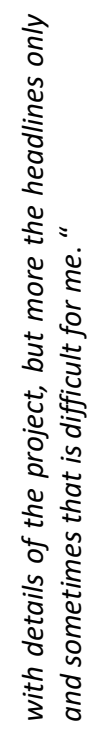 & 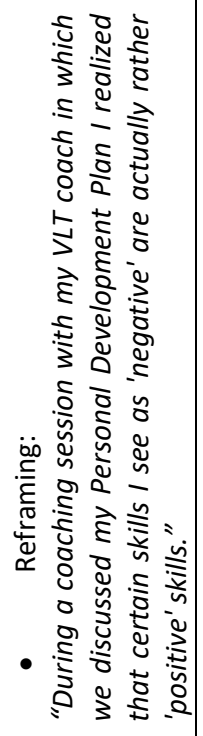 & 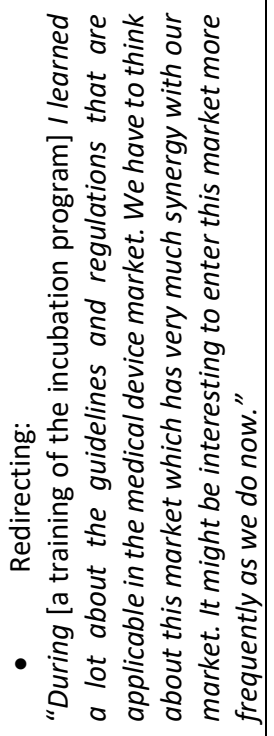 & 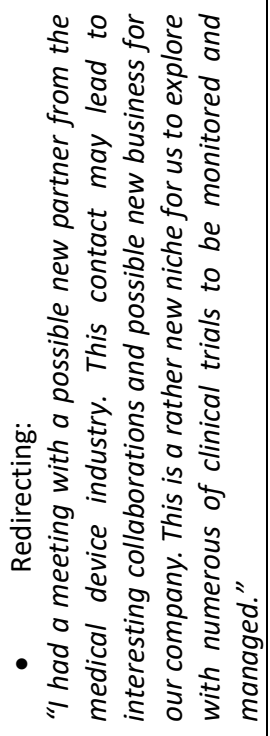 & 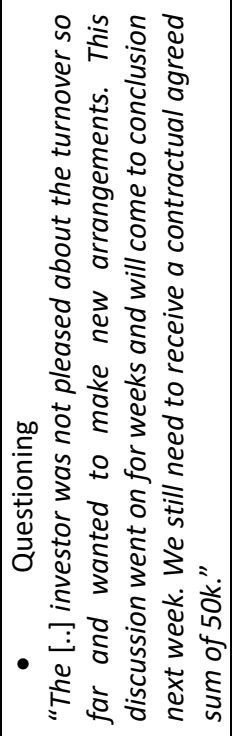 & 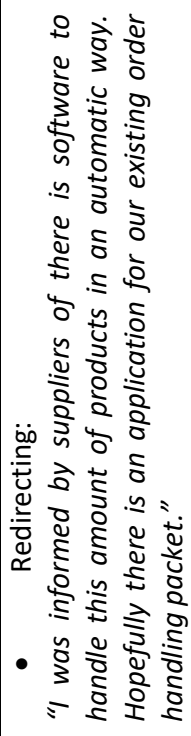 \\
\hline & & 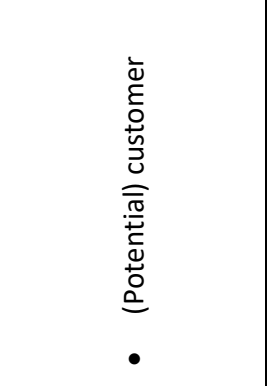 & 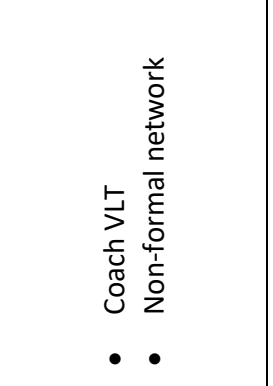 & 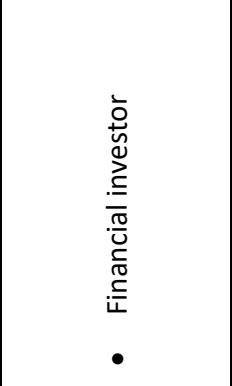 & 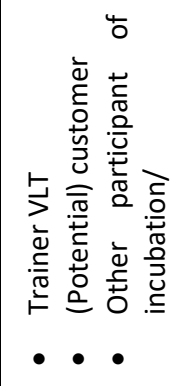 \\
\hline & & ஓ゚ & ১゚ & ஓ゚ & ১े \\
\hline & & 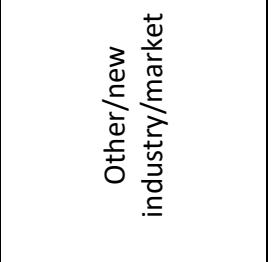 & 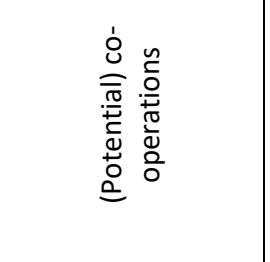 & 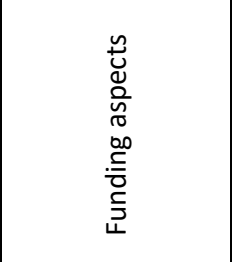 & 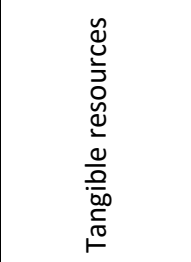 \\
\hline
\end{tabular}




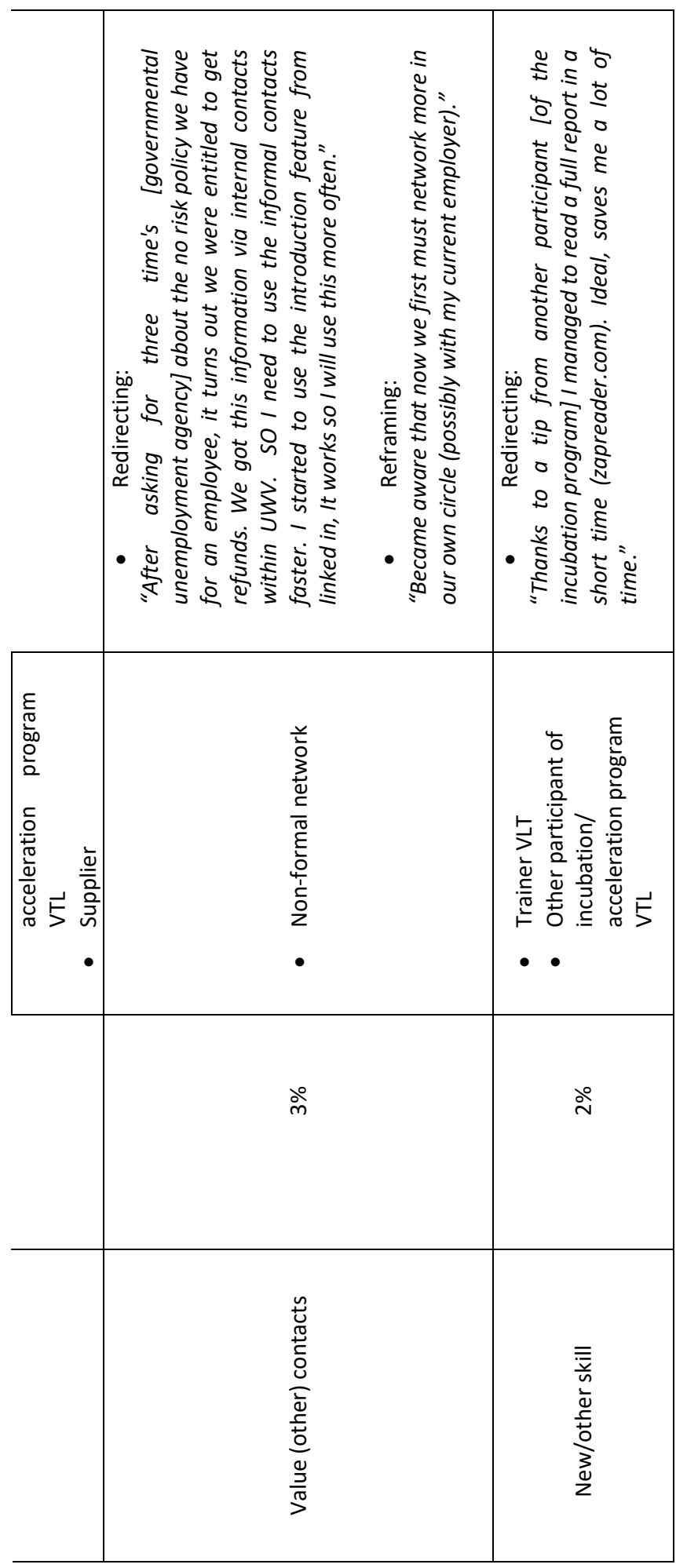


Overall, the most commonly mentioned sensebreaking instances are the result of the redirecting and reframing mechanisms and lead to novel sensemaking of human capital. Redirecting appears to benefit more technical novel sensemaking, whereas reframing leads to more metacognitive development in terms of changing one's attitude or belief towards a particular way of developing the business opportunity. These sensebreaking occurrences lead to novel sensemaking by entrepreneurs of their business plan and business model, as well as of their strategy, in particular related to issues of applicability of the service or product developed.

The findings also show that reframing instances which lead to novel sensemaking are more often initiated by stakeholders in the business incubation/acceleration program than outside it, whereas questioning more commonly occurs in interaction with stakeholders outside the business incubation program. Yet, in terms of novel sensemaking the respondents report very similar themes regardless of the type of stakeholder - with the exception of financial issues and tangible resources which are typically the result of sensebreaking occurrences outside the business incubator.

In Graph 3-4 we show the novel sensemaking themes that entrepreneurs reported per sensebreaking mechanisms. As we can see, redirecting leads to novel sensemaking mainly in terms of application of the business opportunity, the strategy or business plan and changes in the market targeted. Reframing also affects the business strategy, but it affects more general changes in the overall business model as well as the value proposition of the venture. The value proposition is also affected by occurrences of questioning, yet questioning mainly changes - in the sense of enhances - the entrepreneur's customer orientation and his sensemaking of funding. 


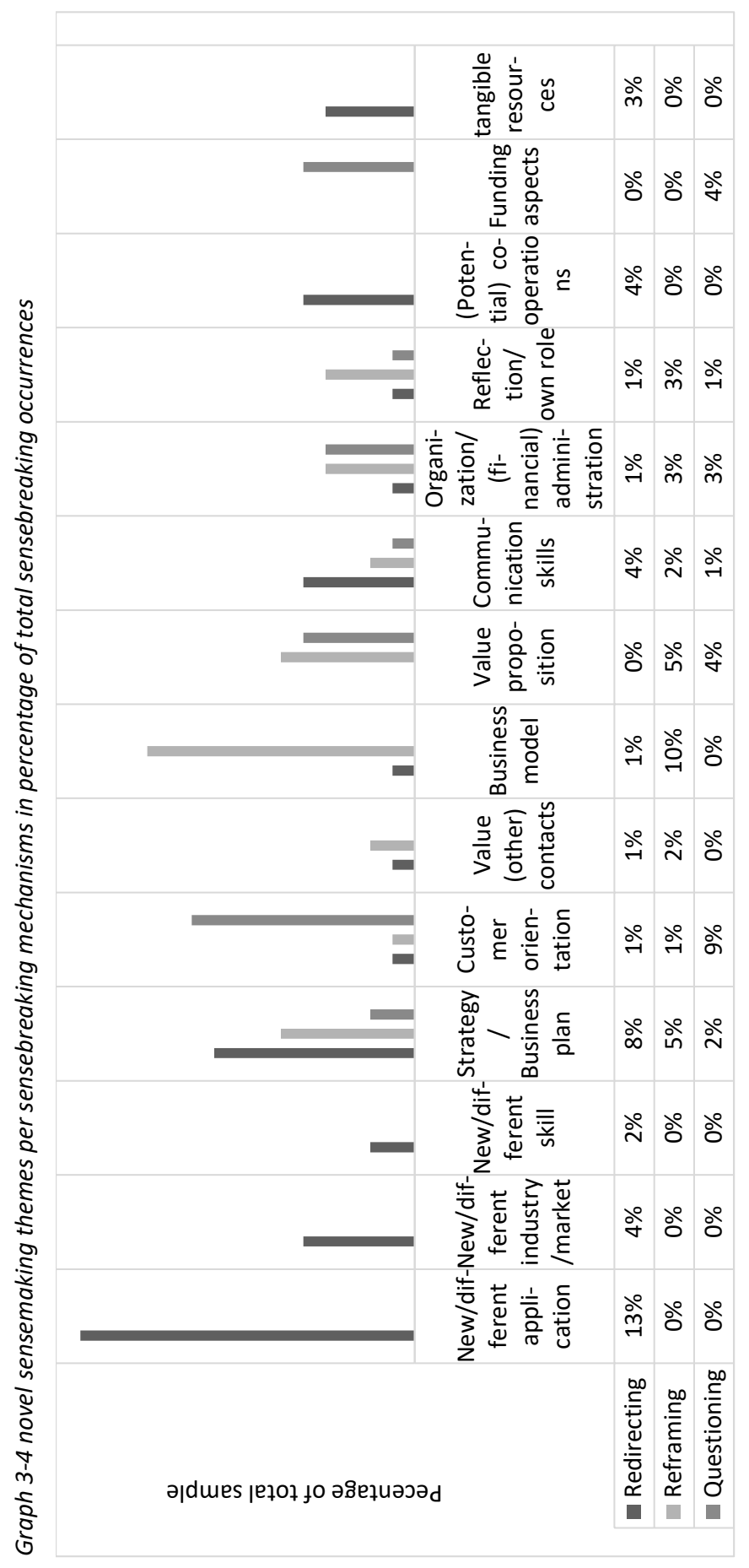




\subsection{CONCLUSION AND DISCUSSION}

The aim of this study is to identify the effects of intersubjective sensebreaking on the development of entrepreneurial cognition. For this we examined how redirecting, reframing and questioning that are initiated by third parties influence novel sensemaking by entrepreneurs during opportunity development. Employing qualitative analysis of diary data, we made two observations: 1) there are differential frequencies and effects of sensebreaking mechanisms on entrepreneurial cognitive development and 2) stakeholder groups involved in sensebreaking affect entrepreneurial cognitive development differently. We finish this section with a more general discussion of sensebreaking in the context of entrepreneurship.

\section{DIFFERENTIAL EFFECTS AND FREQUENCIES OF SENSEBREAKING MECHANISMS ON ENTREPRENEURIAL}

\section{COGNITIVE DEVELOPMENT}

In total we identified 14 different themes in the diaries of respondents which reflect novel sensemaking as a result of sensebreaking. Most novel sensemaking regard human capital. For example, it relates to strategic reconsiderations, changes in business planning and alterations of the entrepreneur's business model or his customer orientation. In addition, sensebreaking leads to cognitive development regarding more awareness of possible applications of the product or service developed, as well as improvement of the entrepreneur's communication skills. Also, novel sensemaking due to sensebreaking occurrences pertain to social capital such as the use of different networks for tangible and intangible support. Sparsely, novel sensemaking concerns economic capital such as loans, housing, machines or (patents or licenses for) technologies and prototypes, in short, everything that has material value and can be sold.

Based on the thematic focus of sensebreaking occurrences reported by entrepreneurs in our sample we conclude that sensebreaking predominantly affects cognitive development in terms of human capital (strategic learning) but also in terms of social capital, such as more appreciation of (the value of) various social networks.

In terms of frequency, redirecting is reported the most often. Redirecting occurrences lead to novel sensemaking of business plan writing, the use of networks and other more technical questions involved in developing a business opportunity. In that way, it leads to cognitive development in terms of knowledge, skills and capability 
formation of individual actors. Reframing, the second most frequently reported sensebreaking mechanism, stimulates entrepreneurs' reflections on and realization, for example the importance of a customer orientation. In that way, reframing affects entrepreneurs' beliefs, attitudes and meta-cognition regarding opportunity development. Questioning is the least reported sensebreaking mechanism, related to team or financial issues associated with opportunity development.

We conclude that there is an important difference in the way that redirecting and reframing affect cognitive development of entrepreneurs. Redirecting instances trigger mainly the (re)consideration of other or new methods, techniques or pieces of information and advice. Reframing on the other hand occurs less in the entrepreneurs' reports however it appears that reframing episodes influence the entrepreneurs in a more principle, profound way. Reframing forces the entrepreneur to reflect on his own thinking, and thus facilitates the development of entrepreneurial cognitive strategies in general. Specifically, reframing raises awareness for value proposition or the business model as a whole, whereas redirecting 'merely' points out certain details or focus points of the business model.

In other words: Reframing sensebreaking triggers the 'WHAT' and 'WHY' questions in business opportunity development and stimulates entrepreneurs' reflections on and realization of something completely different and 'out of the box'; whereas redirecting sensebreaking trigger the 'HOW' questions in business opportunity development and prompt the more technical questions of an otherwise already known or accepted phenomenon. In this way, reframing facilitates the shaping of beliefs and attitudes of entrepreneurs which has been described as deeper level learning (Krueger, 2007).

The deeper level learning is associated with metacognitive skills. Metacognition has been defined by as the ability to understand, control, and reflect upon one's learning (Dennison \& Schraw, 1994). Metacognition captures cognitive processing at a more general, abstract level than cognition (Haynie et al., 2012: p.240) and research has advanced that metacognitive aware learners are more strategic and perform better than unaware learners (Dennison \& Schraw, 1994). It has been argued that metacognition represents the cognitive basis of the "entrepreneurial mindset" (Haynie et al., 2009). 
Our findings suggest that sensebreaking has implications for the development of the entrepreneurial mindset or Krueger's (2007) deeper level learning. The results show that particular stakeholders appear more frequently in providing 'metacognitive resources' - by means of triggering reframing occurrences -, which are seen as the building blocks of one's metacognitive ability and which consist of metacognitive knowledge and metacognitive experience (Haynie et al., 2012). Our results also show that reframing leads to metacognitive learning in terms of novel, intersubjective sensemaking related to metacognitive knowledge and experience which "contribute to "qualifying the implications of thought content" (Sanna \& Schwarz, 2007, p. 173) as applied to a particular problem or situation given what an individual understands about people, tasks, strategy, themselves (intuitions, emotions, experiences, memories), and their own cognitive processes (Schraw \& Dennison, 1994)." (Haynie et al,. 2012: p.241). Those metacognitive processes are related to the 'why' of entrepreneurial action and attitude which, as we found, are triggered by reframing occurrences. Since individuals vary in metacognitive ability, we suggest that the sensebreaking mechanism of reframing represents an important micro-level, social-cognitive tool by which third parties can affect deeper-level learning among entrepreneurs.

On a related note, we saw that some entrepreneurs report reframing occurrences more than others which could be related to what Corbett (2007) calls learning asymmetries among entrepreneurs. According to Corbett, these learning asymmetries "have a profound effect on why some individuals discover entrepreneurial opportunities while others do not." (Corbett, 2007: p. 116). Relating that insight to the importance of deeper level beliefs which are conducive to entrepreneurial learning and subsequently a more expert entrepreneurial mindset we argue that sensebreaking can be a valuable mechanisms for third parties to affect entrepreneurial learning, also on a deeper level by means of reframing, and thus stimulate or accelerate the development of an entrepreneurial mindset, with associated entrepreneurial attitudes, perspectives, and beliefs.

\section{EFFECTS OF DIFFERENT STAKEHOLDERS ON SENSEBREAKING}

The results show that stakeholders often affect more than one sensebreaking mechanism, and their positive effect is enhanced when stakeholder interaction occurs in complementary form. In other words, the more stakeholders are engaged in interaction 
with the entrepreneurs, the more likely he or she reports the occurrences of all three sensebreaking mechanisms.

We also note that not all stakeholder groups contribute to sensebreaking equally. Some stakeholders affect redirecting more than reframing, and vice versa. Redirecting instances are the result of interaction with (potential) customers, while reframing is associated with the formal network, such as governmental institutions and trade fairs, as well as financial investors. Questioning, the least commonly mentioned sensebreaking mechanism, happens mainly as a result of interaction with potential customers, financial investors and, to a lesser extent, also coaches of the VLT business incubation program.

As we saw in the results, the effects of redirecting and reframing mechanisms triggered by coaches and (expert) trainings in terms of novel sensemaking are comparable to those of the non-formal network and (potential) customers. We observe this in particular in relation to human capital associated with the themes business model, value proposition and strategy. We therefore conclude that intersubjective sensebreaking inside a business incubation/acceleration program are at least as frequently reported as those outside a support program.

This means that business support provided in an institutionalized incubation environment can be as powerful in sensemaking processes - at least regarding sensebreaking - as the support provided by an entrepreneur's own network. An explanation for this is that entrepreneurs come to a support program with an explicit intent to learn, ask questions and expose themselves to learning in this institutionalized incubation/acceleration environment. Yet, our findings provide empirical support to the relative importance of incubation program impact on entrepreneurial cognitive development in comparison to the entrepreneur's own (non)formal network.

There are however some differences in the effect of sensebreaking episodes occurring inside and outside the support program. Sensebreaking episodes in the nonformal network and of potential customers are solely focused on the topic of 'added value' or the value proposition, while sensebreaking within the business incubation program typically treats business model as a whole.

Another difference between stakeholders inside and outside the support program regards the different effects of the sensebreaking mechanisms. Reframing is noticeable more frequently mentioned inside the incubation program than outside of it - particularly in association with interaction with coaches and (expert) trainers - while outside the 
incubation program, interaction with stakeholders lead to significantly more questioning occurrences. A possible explanation for this difference is that the business incubation program VLT targets explicitly the improvement - and thus change - of business opportunity in order to speed up entrepreneurial learning and successful commercialization of that opportunity.

Ozgen \& Baron (2007) described how different social sources play a role in providing feedback to entrepreneurs during opportunity identification. This study extends their findings by adding micro-level evidence of the sensebreaking mechanisms involved in this feedback, and how the use of these different mechanisms - and their effects differs depending on which stakeholder is involved in feedback. For example, we saw that reframing more often occurs in a setting which presupposes trust or at least an informal setting, such as with the mentor or with fellow entrepreneurs.

While a business incubation/acceleration program is a formal, institutional environment it integrates non-formal elements such as peer-to-peer interaction, coaching and networking events. On the other hand, a more formal learning context is induced by lectures and trainings, as well as panel presentations. The findings of our study indicate that by providing both types of learning environments - informal and formal - the business incubation/acceleration program stimulates different types of trust which play complementary roles in the development of weak and strong ties (Scarbrough et al., 2013), and subsequent acquisition of (different types of) information relevant for venture development. Studies have found that during opportunity development, strong ties can encourage entrepreneurial persistence (Davidsson \& Honig, 2003) and that trust-based personal relationships enable entrepreneurs to gain greater feedback on their business idea (Greve, 1995). For example, St-Jean \& Audet (2012) found that coaching leads to cognitive as well as metacognitive learning among novice entrepreneurs. This is congruent with our findings and offers and explanation as to why sensebreaking, in the form of reframing occurrences, speeds up entrepreneurial metacognitive learning and the development of a more expert entrepreneurial mindset.

\section{THE CONCEPT OF SENSEBREAKING IN THE CONTEXT OF SOCIALLY SITUATED COGNITION}

The mechanisms of redirecting, reframing and questioning function to provide stakeholders access to an entrepreneur's cognition. The results of this our research thus yield empirical evidence that intersubjective sensebreaking affects the development of entrepreneurial cognition, by triggering novel sensemaking processes mainly regarding human capital and to a lesser extent social and economic capital. 
In that way, sensebreaking empirically demonstrates the workings of socially situated cognition as described by Mitchell et al. (2012). Socially situated cognition emphasizes the situated, embodied and interactive nature of opportunity development in general, and entrepreneurial cognition in particular. This perspective views cognition in its social context, with the idea that agency may be distributed in pursuing entrepreneurial opportunities and in which metacognition, which enables individuals to deal with feedback from a dynamic context, plays an important role (Randolph-Seng et al., 2015).

Socially situation cognition thus emphasizes the distributed nature of cognition, in terms of other actors being the source of information and knowledge storage which can be leveraged by the entrepreneurs for venture development (Dew et al., 2015). By means of sensebreaking mechanisms stakeholders affect novel sensemaking processes of entrepreneurs, which supports the idea that much entrepreneurial activity involves learning by entrepreneur who are understood as not working alone, but interactively and collectively (Dew et al., 2015).

Stakeholders are crucial for distributed (socially situated) cognition because cognition is considered a system-level property which arises among the actors that constitute a social system. (Dew et al., 2015), in this case the entrepreneur and relevant stakeholders in the business opportunity development process.

Our findings regarding the role of sensebreaking mechanisms in shaping entrepreneurial sensemaking also provide empirical underpinnings for what has been called 'transactive memory' (Zheng, 2012) which provides a conceptual, system-level notion of thinking about sensemaking intersubjectively. It is defined as the cognitive interdependence of actors and which leads to a cognitive division of labor (Dew et al., 2015). The notion of transactive memory is in line with and supported by our findings of sensebreaking mechanisms as socially situated cognitive processes.

Of particular importance in this context is the interactive nature of sensebreaking which invariably involves language, namely feedback provided to the entrepreneur by means of reframing, redirecting or questioning occurrences. Language is a central notion in socially situated cognition as Cornelissen \& Clarke (2011) propose. They authors argue that external speech reconfigures ideas to fit the demands of spoken language which organizes thinking itself, in order to meet the demands of linguistic encoding 
(Cornelissen \& Clarke, 2011). Sensebreaking is therefore an important micro-level mechanism when using language because "when an entrepreneur gets feedback from stakeholders who display whether they understand and accept the intentions for the venture, it allows the entrepreneur to validate that understanding or to correct it, and this, in turn, has consequences for the way in which the understanding of the venture evolves" (Cornelissen \& Clarke, 2011: p. 777).

We therefore propose that the concept of sensebreaking is indispensable for the discourse about the role of socially situated cognition in opportunity development. Our findings show that reframing, redirecting and questioning are employed by stakeholders and simultaneously leveraged by entrepreneurs in concrete, every-day (linguistic) interactions shape entrepreneurial cognition and the 'entrepreneurial mindset, facilitating the development a business opportunity by means of novel entrepreneurial sensemaking processes.

We conclude that sensebreaking represents as a socially situated, cognitive mechanism which offers an instrument to stakeholders to influence an entrepreneur's cognition during venture development.

\subsection{CONTRIBUTION}

This study contributes in the following ways. Firstly, our study yields empirical evidence of the intersubjective nature of micro-decisions by entrepreneurs regarding various aspects of opportunity development, such as business model design, strategy formation and the value of networks. These findings provide valuable empirical insights into the micro-level processes of the situated, distributed nature of cognition as conceptualized by West (2007) and McMullen (2014). In particular, McMullen \& Dimov (2013) emphasize the importance of analyzing micro-decisions by entrepreneurs which pertain to questions of whether and how to continue investing into the development of a business opportunity. They argue that these decisions involve the necessity to take into account the potential preferences of stakeholders which he associates with sensemaking - but which, they find, is difficult study empirically. In fact, our study yields empirical evidence that sensebreaking as an interactive process is associated with different cognitive outcomes, depending on the kind of stakeholder involved - as we saw in the 
difference between stakeholders inside and outside the business incubation/acceleration program with regards to reframing and questioning occurrences.

Secondly, we contribute to the conceptualization of entrepreneurial sensemaking as the result of a social cognitive process. The distinction between the roles that redirecting, reframing and questioning play, as well as the varying use of these sensebreaking mechanisms by a number of stakeholders and their respective cognitive outcomes contribute to our understanding of how input by third parties affects entrepreneurial cognition during opportunity development. Various authors have pointed out that there is a lack of studies in the area of what has been termed (socially) situated cognition (Mitchell et al., 2012; Dew et al., 2015). For example, Shepherd (2015) only recently emphasized the need for using an interactive perspective when examining entrepreneurial opportunity identification and preparation. We showed that sensebreaking is the results of interactions with various stakeholders which trigger the entrepreneurs to question, reframe and redirect their existing notions on venture building and thus function as an antecedent to entrepreneurial cognition.

From these results we conceptualize sensebreaking as a socially situated cognitive mechanism in the identification and preparation of a business opportunity, by triggering novel sensemaking processes regarding the entrepreneur's human, social and economic capital, and geared towards making the opportunity more commercially viable. Particularly we saw how certain stakeholders enable entrepreneurs to acquire metacognitive skills by means of triggering reframing instances. In that way, sensebreaking is a mechanism in progressing entrepreneurial cognition towards a more 'expert' entrepreneurial mindset. These insights advance current theory building efforts on the role of socially situated cognition in the development of meta-cognition among entrepreneurs. Our study thus contributes to theory-building on the role of socially situated cognitive mechanisms by which the community affects entrepreneurial (meta)cognitive development.

Thirdly, the relatively novel data collection method of analyzing diary entries enables us to observe and report on the origins of entrepreneurial cognition with very limited retrospection bias. By doing so, we answer to Shepherd's (2015) call for methodological experimentation and modernisation in scientific entrepreneurship research. By means of the diary method we observed real-time, individual-level interactions between entrepreneurs and specific stakeholders over time, as well as their 
effects on the cognitive development of entrepreneurs. We suggest that using this method helps to avoid methodological pitfalls in qualitative research on the phenomenon of entrepreneurship, such as socially desirable or retrofitted answers by entrepreneurs under investigation. Also and more generally, we suggest that the diary method contributes to expanding the array of research methods in entrepreneurship, in particularly on the micro-level interactions which affect opportunity development.

Fourthly and more practically, sensebreaking actions are also useful in clarifying our existing understanding of role of critical feedback provided by different stakeholders. Our study yields insights into the functions of institutional support program - particularly, in providing a demonstration of the influence of different program staff - mentors or trainers - on reframing and redirecting mechanisms triggered by intersubjective sensebreaking. We found that reframing can prove valuable in creating 'cognitive space' for novel assessments and subsequent decision-making about the value of a resource. Meanwhile, redirecting facilitates incremental learning episodes, such as shifting the entrepreneur's attention to a different market or a novel partner in value creation.

Institutionalized or informal support program can benefit from applying the insights from this study for more effective - and efficient - opportunity development, for example by providing interaction with mentors and coaches respectively trainers according to the perceived need of the entrepreneur for reframing and redirecting occurrences and their associated effects on cognitive development by the entrepreneur. Based on the results of this study we suggest that ultimately intersubjective sensebreaking leads to decisionmaking which has a greater 'emphatic accuracy' (McMullen, 2013) and subsequently to better stakeholder involvement and greater chance of successful business opportunity development.

\subsection{LIMITATIONS}

There are some limitations to the generalizability of the results presented in this study, of which the three main ones are discussed in turn.

For one, the data collection method is not without limitations. Diary data is a very 'raw' form of data; the entrepreneurs were free to write down thoughts about their development, only guided by the four 'topics' within each weekly diary. The result is 
rather chaotic data; sometimes, sentences are not finished, various issues are mentioned in general without going into relevant details or names of the parties involved. Regretfully, applications of diary methods are scarce in the entrepreneurial literature with the exception of Kato and Wiklund (2011) who analyzed blog entries of entrepreneurs. Therefore we could not turn to other studies for insights into legitimate ways of doing diary studies. However and on a more positive note, the resulting real-time data also makes it a very pure and authentic form of research data.

The commitment of the entrepreneurs was an important factor in getting the data collection method to work. Entrepreneurs varied in terms of content and frequency of self-reporting. We did notice that some entrepreneurs enjoyed the exercise; while others underlined and at times critized the time commitment and discipline required for consistent self-reporting given the fact that their new venture kept them endlessly busy. This we found appeared to affect the level of detail and elaboration with which the diaries were kept. Also, some entrepreneurs seemed cautious about which information to report in the diaries, possibly for reasons of confidentiality and secrecy. In order to provide a maximum level of comfort to the entrepreneurs, all information given in the diaries were maintained in secure servers with very limited access to the employees at the business accelerator.

Another limitation is that we did not code episodes of sensebreaking which are triggered by critical self-reflection and introspection by the entrepreneur, due our interest in intersubjective sensebreaking by means of stakeholder interaction. Neither could we focus on sensebreaking occurrences involving public agencies (regulations, policies, laws or sanctions), influence of books, magazines and other media sources. These were not considered (though mentioned by the entrepreneurs a few times) for lack of explicit stakeholder source but which could possibly affect entrepreneurial cognition.

Finally, we studied a biased sample of entrepreneurs. Our data was drawn exclusively from diaries of entrepreneurs in a business support environment, limiting it to help-seeking entrepreneurs. They are therefore a 'self-selected' group in terms of openness to learning which could lead to the outcomes of this study being biased towards reported learning. This means that the results cannot be representative, and we are therefore unable to draw general, population-wide conclusions. On a related note, the size of our sample was a limitation in itself. Certainly additional participants would make our results more robust. This is a constraint often encountered in qualitative research. 


\subsection{FUTURE RESEARCH}

On the basis of our results, we identify three promising research avenues for future studies regarding the concept of sensebreaking.

One, our study focused on the sensebreaking mechanisms of reframing, redirecting and questioning. That makes this study descriptive in nature instead of explanatory. In future research, a focus on the explanatory dimension of sensebreaking could contribute to the understanding of sensebreaking in venture development. For example, the relationship between the frequency of sensebreaking occurrences on the one hand and survival and growth of a venture on the other could yield important information about the effect of sensebreaking mechanisms on the commercial success of that venture.

A second relevant area of research is the relationship between emotion and sensebreaking. Shepherd (2015) emphasizes the role of emotions in cognitive development of entrepreneurs, and the relevance of research on this topic. We showed the sensebreaking mechanisms trigger novel sensemaking processes entrepreneurs to acquire cognitive skills. Particularly interesting in this context the role that passion plays in enabling sensebreaking episodes. Cardon et al. (2009) argue that passion is a critical element in an entrepreneur's, because it enhances commitment and keeps the energy focused on his achieving his goals. Also, the authors point to the role that passion plays in the entrepreneur's identification with his role and tasks as entrepreneur. The identification with the entrepreneurial role also plays a role in sensebreaking, because it is associated with reframing occurrences found in this study. Concretely, we saw that reframing instances led to changing perspectives of the entrepreneur, characteristically towards a more 'entrepreneurial' mindset; a concern with business development under conditions of uncertainty. As we saw in the results, reframing can lead to meta-cognitive or 'deeper level learning' (Krueger, 2007), in terms of enabling the novel sensemaking such as the adoption of different perspectives. Future empirical examination of the role of entrepreneurial passion in sensebreaking effects, in particular of reframing occurrences, contributes to understanding the antecedents to deeper level' learning and the development of an entrepreneurial mindset.

A third area of future research is trust which is a relevant factor in sensebreaking and worth exploring. In general, trust is relevant for the integration of weak and strong 
tie networks (Scarborough et al., 2013), and could ultimately affects the access to and frequency of sensebreaking occurrences. Bammens \& Collewaert (2014) found that trust plays a role in entrepreneur-angel investor relationships, both positively and negatively. Financial investors are a relevant party for exposing entrepreneurs to sensebreaking instances; we saw in the results that investors affect novel entrepreneurial sensemaking, most notably in terms of questioning occurrences. It might be a fruitful endeavor for the future to study the level of trust between certain stakeholders such as investors and entrepreneurs who are relevant for sensebreaking, or examine the effect of different forms of trust type - such as described by Scarbrough et al. (2013) - on the occurrences and effects of sensebreaking.

Taking these research avenues in consideration, we posit that sensebreaking has a promising because rich potential to enlarge the conceptual toolkit for analyzing the effect of interactions on the development of entrepreneurial cognition.

\subsection{BIBLIOGRAPHY}

Bammens, Y. and V. Collewaert (2014). Trust Between Entrepreneurs and Angel Investors: Exploring Positive and Negative Implications for Venture Performance Assessments. Journal of Management 40(7): 1980-2008.

Barney, J. (1991). Firm resources and sustained competitive advantage. Journal of management, 17(1), 99-120.

Baron, R. A. (2004). The cognitive perspective: a valuable tool for answering entrepreneurship's basic "why" questions. Journal of Business Venturing, 19(2): 221239.

Baron, R.A., and Ward, T.B. (2004). Expanding entrepreneurial cognition's toolbox: Potential contributions from the field of cognitive science. Entrepreneurship Theory and Practice, 28(6): 553-573.

Baron, R.A. (2006). Opportunity recognition as pattern recognition: How entrepreneurs "connect the dots" to identify new business opportunities. Academy of Management Perspectives, 1(20): $104-119$. 
Baron, R. A. (2007). Behavioral and cognitive factors in entrepreneurship: entrepreneurs as the active element in new venture creation. Strategic Entrepreneurship Journal, 1(12): 167-182.

Baron, R. A., \& Ensley, M. D. (2006). Opportunity recognition as the detection of meaningful patterns: Evidence from comparisons of novice and experienced entrepreneurs. Management science, 52(9), 1331-1344.

Bettiol, M., Di Maria, E., \& Grandinetti, R. (2011). Market extension and knowledge management strategies of knowledge-intensive business services. Knowledge Management Research \& Practice, 9(4), 305-314.

Bingham, C. B., \& Eisenhardt, K. M. (2011). Rational heuristics: the 'simple rules' that strategists learn from process experience. Strategic Management Journal, 32(13), 14371464.

Bolger, N., Davis, A, Rafaeli E. (2003). Diary methods: Capturing life as it is lived. Annual Review of Psychology, 54(1): 579-616.

Borchert, P. S., \& Rochford, L. (2009). The role of negative feedback in the process of opportunity exploitation. Frontiers of Entrepreneurship Research, 29(13), 1.

Cardon, M. S., Wincent, J., Singh, J., \& Drnovsek, M. (2009). The nature and experience of entrepreneurial passion. Academy of management Review, 34(3), 511-532.

Carsrud, A. L., \& Brännback, M. (2009). Cognitive maps in entrepeneurship: Researching sense making and action In A. L. Carsrud, \& M. Brännback, (Eds.), Understanding the entrepreneurial mind: Opening the black box, International Studies in Entrepreneurship 24, (pp. 75 -96). Springer Science \& Business Media.

Coleman, J. S. (1988). Social capital in the creation of human capital. American journal of sociology, S95-S120.

Corbett, A. C. (2007). Learning asymmetries and the discovery of entrepreneurial opportunities. Journal of Business Venturing, 22(1), 97-118.

Cornelissen, J. P., Clarke, J. S., \& Cienki, A. (2012). Sensegiving in entrepreneurial contexts: The use of metaphors in speech and gesture to gain and sustain support for novel business ventures. International Small Business Journal, 30(3), 213-241. 
Davidson, D. (2001). Subjective, Intersubjective, Objective. New York: Oxford University Press.

Davidsson, P., \& Honig, B. (2003). The role of social and human capital among nascent entrepreneurs. Journal of business venturing, 18(3), 301-331.

Elio, R. and Scharf, P. B. (1990). Modeling novice-to-expert shifts in problem-solving strategy and knowledge organization. Cognitive Science, 14( 4): 579-639.

Flick, U. (2007). Managing quality in qualitative research. Sage: London, Thousand Oaks, New Delhi.

Florin, J., Lubatkin, M., \& Schulze, W. (2003). A social capital model of high-growth ventures. Academy of Management Journal, 46(3), 374-384.

Forbes, D. P. (2005). Are some entrepreneurs more overconfident than others? Journal of Business Venturing, 20(5): 623-640.

Foss, N. J., Klein, P. G., Kor, Y. Y., \& Mahoney, J. T. (2008). Entrepreneurship, subjectivism, and the resource-based view: toward a new synthesis. Strategic Entrepreneurship Journal, 2(1), 73-94.

Gartner, W.B., Shaver, K.G., Gatewood, E.J., Katz, J.A. (1994). Finding the entrepreneur in entrepreneurship. Entrepreneurship: Theory and Practice, 18(3): 5-9.

Gemmell, R.M., Boland, R.J., Kolb, D.A. (2012). The socio-cognitive dynamics of entrepreneurial ideation. Entrepreneurship: Theory and Practice, 36(5): 1053-1073.

Gioia, D. A. and Chittipeddi, K. (1991). Sensemaking and sensegiving in strategic change initiation. Strategic Management Journal, 12(6): 433-448.

Grégoire, D. A., Corbett, A.C., McMullen, J.S. (2011). The cognitive perspective in entrepreneurship: An Agenda for Future Research. Journal of Management Studies, 48(6): 1443-1477.

Grégoire, D. A., \& Shepherd, D. A. (2012). Technology-market combinations and the identification of entrepreneurial opportunities: An investigation of the opportunityindividual nexus. Academy of Management journal, 55(4), 753-785. 
Greve, A. (1995). Networks and entrepreneurship_an analysis of social relations, occupational background, and use of contacts during the establishment process. Scandinavian journal of management, 11(1), 1-24.

Haynie, J. M., Shepherd, D. A., \& McMullen, J. S. (2009). An opportunity for me? The role of resources in opportunity evaluation decisions. Journal of Management studies, 46(3), 337-361.

Haynie, J. M., Shepherd, D., Mosakowski, E., Earley, P.C. (2010). A situated metacognitive model of the entrepreneurial mindset. Journal of Business Venturing, 25(2): 217-229.

Haynie, J. M., Shepherd, D. A., \& Patzelt, H. (2012). Cognitive adaptability and an entrepreneurial task: The role of metacognitive ability and feedback. Entrepreneurship Theory and Practice, 36(2), 237-265.

Hill, R. C. and Levenhagen, M., 1995. Metaphors and mental models: Sensemaking and sensegiving in innovative and entrepreneurial activities. Journal of Management, 21(6): 1057-1074.

Hill, C. E., Thompson, B. J., and Williams, E. N. (1997). A guide to conducting consensual qualitative research. The Counseling Psychologist, 25: 517- 572.

Hindle, K., 2004. Choosing qualitative methods for entrepreneurial cognition research. A canonical development approach. Entrepreneurship Theory and Practice, 28(6): 575607.

Holt, R., \& Macpherson, A. (2010). Sensemaking, rhetoric and the socially competent entrepreneur. International Small Business Journal, 28(1), 20-42.

Jarillo, J. C. (1989). Entrepreneurship and growth: The strategic use of external resources. Journal of Business Venturing, 4(2), 133-147.

Kato, S. and Wiklund, J. (2011). Doing good to feel good - A theory of entrepreneurial action based in hedonic psychology. Frontiers of Entrepreneurship Research, 31(4): 123 $-137$.

Kolodner, J. (1983). Towards an understanding of the role of experience in the evolution from novice to expert. International Journal of Man-Machine Studies, 19(5): 497-518. 
Kraaijenbrink, J., Spender, J. C., \& Groen, A. J. (2010). The resource-based view: a review and assessment of its critiques. Journal of management, 36(1), 349-372.

Krueger Jr, N. F. (2003). The cognitive psychology of entrepreneurship. In Handbook of entrepreneurship research (pp. 105-140). Springer US.

Krueger, N. F. (2007). What Lies Beneath? The experiential essence of entrepreneurial thinking. Entrepreneurship Theory and Practice, 31(1): 123-138.

Kor, Y. Y., Mahoney, J. T., \& Michael, S. C. (2007). Resources, capabilities and entrepreneurial perceptions. Journal of Management Studies, 44(7), 1187-1212.

Lim, J.Y.-K., Busenitz, L.W., Chidambaram, L. (2013). New venture teams and the quality of business opportunities identified: Faultlines between subgroups of founders and investors. Entrepreneurship Theory and Practice, 37 (1), 47-67.

Lawrence, T. B., and Maitlis, S. (2005). The disruption of accounts: Sensebreaking in organizations. Paper presented at the Academy of Management Annual Meeting, Honolulu, $\mathrm{HI}$.

Maclntosh, R., and Beech, N. (2010). The practice of failure: sensebreaking dialogues and the stymieing of change. In: 70th Annual Meeting of the Academy of Management 2010, Montreal, Quebec, Canada.

Maitlis, S., \& Lawrence, T. B. (2007). Triggers and enablers of sensegiving in organizations. Academy of management Journal, 50(1), 57-84.

Mantere, S., Schildt, H., and Sillince, John A.A. (2009). Managing false starts: managerial sensegiving and sensebreaking in response to an interrupted strategic change effort. In: Academy of Management Conference, Chicago, USA.

McKelvie, A., \& Davidsson, P. (2009). From resource base to dynamic capabilities: an investigation of new firms. British Journal of Management, 20(s1), S63-S80.

McKelvie, A., Haynie, J. M., Gustavsson, V. (2011). Unpacking the uncertainty construct: Implications for entrepreneurial action. Journal of Business Venturing, 26(3): 273-292.

McMullen, J. S. and Shepherd, D. A. (2006). Entrepreneurial action and the role of uncertainty in the theory of the entrepreneur. Academy of Management Review, 31(1): 132-152. 
McMullen, J. S., \& Dimov, D. (2013). Time and the entrepreneurial journey: The problems and promise of studying entrepreneurship as a process. Journal of Management Studies, 50(8), 1481-1512.

Van Merriënboer, J. J. G., Schuurman, J. G., De Croock, M. B. M., \& Paas, F. G. W. C. (2002). Redirecting learners' attention during training: Effects on cognitive load, transfer test performance and training efficiency. Learning and Instruction, 12(1), 11-37.

Mitchell, R. K., Busenitz, L., Lant, T., McDougall, P. P., Morse, E. A., \& Smith, J. B. (2002). Toward a theory of entrepreneurial cognition: Rethinking the people side of entrepreneurship research. Entrepreneurship theory and practice, 27(2), 93-104.

Mitchell, R. K., Randolph-Seng, B., \& Mitchell, J. R. (2011). Socially situated cognition: Imagining new opportunities for entrepreneurship research. Academy of Management Review, 36(4), 774-776.

Mitchell, D. E., \& Nielsen, S. Y. (2012). Internationalization and globalization in higher education. INTECH Open Access Publisher.

Nahapiet, J., \& Ghoshal, S. (1998). Social capital, intellectual capital, and the organizational advantage. Academy of management review, 23(2), 242-266.

Ozgen, E. and Baron, R. A. (2007). Social sources of information in opportunity recognition: Effects of mentors, industry networks, and professional forums. Journal of Business Venturing, 22(2): 174-192.

Pratt, M. G. (2000). The good, the bad, and the ambivalent: Managing identification among Amway distributors. Administrative Science Quarterly, 45(3): 456-493.

Randolph-Seng, B., Mitchell, R. K., Vahidnia, H., Mitchell, J. R., Chen, S., \& Statzer, J. (2015). The Microfoundations of Entrepreneurial Cognition Research: Toward an Integrative Approach. Foundations and Trends (R) in Entrepreneurship, 11(4), 207-335.

Robinson, P. B., \& Sexton, E. A. (1994). The effect of education and experience on selfemployment success. Journal of business Venturing, 9(2), 141-156.

Sanna, L. J., \& Schwarz, N. (2007). Metacognitive experiences and hindsight bias: It's not just the thought (content) that counts!. Social Cognition, 25(1), 185. 
Sarasvathy, S. D. (2001). Causation and effectuation: Toward a theoretical shift from economic inevitability to entrepreneurial contingency. Academy of management Review, 26(2), 243-263.

Sarasvathy, S. D., Dew N., Read, S., Wiltbank, R. (2008). Designing organizations that design environments: Lessons from entrepreneurial expertise. Organization Studies, 29(3): 331-350.

Scarbrough, H., Swan, J., Amaeshi, K., \& Briggs, T. (2013). Exploring the Role of Trust in the Deal-Making Process for Early-Stage Technology Ventures. Entrepreneurship Theory and Practice, 37(5), 1203-1228.

Schraw, G., \& Dennison, R. S. (1994). Assessing metacognitive awareness. Contemporary educational psychology, 19(4), 460-475.

Shepherd, D. A. (2009). Grief recovery from the loss of a family business: A multi- and meso-level theory. Journal of Business Venturing, 24(1): 81-97.

Shepherd, D. A., Williams, T. A., \& Patzelt, H. (2015). Thinking about entrepreneurial decision making review and research agenda. Journal of management, 41(1), 11-46.

Silverman, D. (2001). Interpreting qualitative data: Methods for analysing talk, text and interaction. London: Sage.

Sirmon, D. G., Hitt, M. A., \& Ireland, R. D. (2007). Managing firm resources in dynamic environments to create value: Looking inside the black box. Academy of management review, 32(1), 273-292.

Spillane, J. P., Reiser, B. J., \& Reimer, T. (2002). Policy implementation and cognition: Reframing and refocusing implementation research. Review of educational research, 72(3), 387-431.

St-Jean, E. and Audet, J., 2012. The role of mentoring in the learning development of the novice entrepreneur. International Entrepreneurship and Management Journal, 8 (1): 119-140.

Thomas, J. B., Clark, S. M., \& Gioia, D. A. (1993). Strategic sensemaking and organizational performance: Linkages among scanning, interpretation, action, and outcomes. Academy of Management journal, 36(2), 239-270. 
Thompson, E. (2007). Mind in life: biology, phenomenology, and the sciences of mind. Harvard University Press: Cambridge, MA.

Thompson, E., \& Stapleton, M. (2009). Making sense of sense-making: Reflections on enactive and extended mind theories. Topoi, 28(1), 23-30.

Venkataraman, S., Sarasvathy, S., Dew, N., Forster, W. R. (2012). Reflections on the 2010 AMR Decade Award: Whither the promise? Moving forward with entrepreneurship as a science of the artificial. Academy of Management Review, 37(1): 21-33.

Vlaar, P. W. L., Van Fenema, P.C., Tiwari, V. (2008). Co-creating understanding and value in distributed work: how members of onsite and offshore vendor teams give, make, demand, and break sense. MIS Qarterly, 32(2): 227-255.

Weick, K. (1969). The social psychology of organizing. Addison-Wesley: Reading, MA.

Weick, K. E. (1995). Sensemaking in organizations (Vol. 3). Sage.

Weick, K. E., Sutcliffe, K. M., \& Obstfeld, D. (2005). Organizing and the process of sensemaking. Organization science, 16(4), 409-421.

Weick, K. E. (2012). Organized sensemaking: A commentary on processes of interpretive work. Human Relations, 65(1), 141-153.

West, G. P. (2007). Collective cognition: When entrepreneurial teams, not individuals, make decisions. Entrepreneurship Theory and Practice, 31(1): 77-102.

Wheeler, L., \& Reis, H. T. (1991). Self-recording of everyday life events: Origins, types, and uses. Journal of personality, 59(3), 339-354.

Wood, M. S., \& McKinley, W. (2010). The production of entrepreneurial opportunity: a constructivist perspective. Strategic Entrepreneurship Journal, 4(1), 66-84.

Zheng, Y. (2012). Unlocking founding team prior shared experience: A transactive memory system perspective. Journal of Business Venturing, 27(5), 577-591.

Zott, C., \& Huy, Q. N. (2007). How entrepreneurs use symbolic management to acquire resources. Administrative Science Quarterly, 52(1), 70-105. 


\section{CHAPTER 4}

\section{Sensemaking beyond the Customer: A cognitive Perspective on MARKET ORIENTATION DEVELOPMENT}

Gabi A. Kaffka, University of Twente

Jeroen Kraaijenbrink, University of Twente

Aard J. Groen, University of Twente

History of the manuscript: This chapter is a modified version of a peer-reviewed paper that was presented at the ASQ-OMT-INSEAD conference in January 2013 in Singapore. 


\section{ABSTRACT}

This study focuses on the development of a market orientation among individual entrepreneurs. Taking a grounded theory approach, we analyze the cognitive development of a market orientation among entrepreneurs in terms of their use of market-oriented mental models during business opportunity development. For this we draw on qualitative data from interviews with 50 entrepreneurs involved in business opportunity development. Our findings show that market-oriented mental models relate predominantly to a broader stakeholder orientation, and appear to shift from activityrelated mental models among novice entrepreneurs to more generic mental schemas related to market-oriented problem solving identified in the reports of experienced entrepreneurs. The results of this study yield insights into the development of a market orientation, in terms of activating the entrepreneurs' awareness of the role of stakeholders as well as encouraging interaction with those stakeholders. With these findings our study contributes to a better understanding of micro-level foundations of a venture's market orientation.

\subsection{INTRODUCTION}

A market orientation reflects a firm's commitment to the creation of (superior) value for customers (Narver et al, 1998). A market orientation is positively linked to performance and enhances an firm's positional advantage (Hult \& Ketchen, 2001; Kirca et al., 2005; Morgan, Vorhies \& Mason, 2009). Studies have also found that a market orientation or focus is beneficial to the performance of start-ups, small and medium-sized businesses (Raju et al, 2011; Ghosh et al., 2001) and gives small companies a potential competitive advantage over larger firms (Reijonen et al., 2012)

The existence of a market orientation on the micro-level is not self-evident, in particular among people who first develop a business opportunity. Few entrepreneurs know what their market is, who their (potential) customers might be (Blank, 2005). They are liable to focusing too much on the technology of their product or service when they develop a business opportunity, at the detriment of focusing on value for the customer (Sarin \& Mohr, 2008; Schindehutte, Morris \& Kocak, 2008; West \& Noel, 2009; Roersen et al., 2013). While a market orientation is ultimately expressed in attitudes and behavior 
regarding the creation and delivery of (superior) customer value (Van Raaij \& Stoelhorst, 2008), it is rooted cognitive faculties, for example in market-oriented information processing activities (Kohli \& Jaworski, 1990) or cognitive models (Morgan et al, 2009).

In this study, we explore the cognitive nature of market orientation development among entrepreneurs. Using grounded theory, we draw on qualitative data from interviews with 50 entrepreneurs engaged in business opportunity development and examine their sensemaking processes related to problem solving during (superior) customer value creation, capture and delivery. Our analysis yields a number of marketoriented mental models which entrepreneurs develop during opportunity development. These results show that the market-oriented mental models which are developed relate predominantly to a broader stakeholder orientation, and appear to shift from activityrelated mental models to more generic mental schemas of market-oriented problem solving with increasing entrepreneurial experience.

The findings of our study show that a market orientation on the micro-level is a socially situated cognitive phenomenon: a market orientation among individuals develops mainly in terms of a growing awareness for the social context, in terms of relevant networks, in the creation of (superior) customer value. The results contribute to a better understanding of the micro-level cognitive development of a market orientation, seen as a relevant research and policy object (Shane, 2012). In addition and more practically, insights from this study can be used to optimize venture development in business support programs, for example with trainings regarding the development of entrepreneurs' market orientation.

We start this paper with a discussion of the key theoretical concepts before turning to methodological aspects. Then we present the results of our analysis, discuss their implications and suggest alleys for future research.

\subsection{THEORY}

\section{MARKET ORIENTATION}

A market orientation has been examined from two theoretical perspectives, the behavioral perspective and the cultural perspective (Becker \& Homburg, 1999). Both perspectives are concerned with "all of the activities in acquiring information" (Narver \& Slater, 1990: p. 21) related to customer needs and preferences. The behavioral 
perspective is concerned with behavior related to market-oriented activities: information generation, interpretation and use for marketing and sales activities (Kohli \& Jaworski, 1990). The focus is clearly on action, as Rijonen et al. (2012) put it. The cultural perspective on a firm's market orientation holds that a market orientation is a' 'business culture' which reflects the commitment to the creation, capture and delivery of superior customer value and which consists of three elements: a customer orientation, a competitor orientation and interfunctional coordination (Slater \& Narver, 1995).

Existing studies suggest that a market orientation in entrepreneurial ventures is beneficial for organizational performance. Raju et al. (2011) use concepts from both the cultural and behavioral perspective in a study of market orientation in small and mediumsized businesses and finds market orientation to be positively related to the performance of small and medium-sized enterprises (Raju et al., 2011). And Boso et al. (2013) found that entrepreneurial firms combining high levels of a market orientation (and of an entrepreneurial orientation) with well-developed network ties display greater performance benefits.

\section{THE DEVELOPMENT OF A MARKET ORIENTATION FROM A COGNITIVE PERSPECTIVE}

Both the behavioral and the cultural perspective on market orientation see marketoriented actions as interpretative actions that are shaped by an individual's cognitive faculties. Cognitive faculties or structures play an important role in entrepreneurial opportunity development by facilitating interpretation, decision-making and problem solving processes (Baron, 2004; Mitchell et al., 2002; Gregoire et al., 2010). They are knowledge structures which help people to make sense of the world, for example by offering interpretative frames and reflecting our priorities (Hill \& Levenhagen, 1995; Krueger, 2003; Weick et al., 2005) and guide interpretation (Harris, 1994). Entrepreneurial cognition has been recognized as a promising avenue for research on entrepreneurial interpretation, assessments, judgments and decisions involved in opportunity evaluation, preparation and exploitation (Mitchell et al., 2002; Mitchell et al., 2007).

A market orientation involves cognitive structures since it links to information generation, processing and storing activities about the market and how to (better) create customer value (Kohli \& Jaworski, 1990) and is concerned with the processing of market information (Hult et al., 2005; Sinkula, 1994). For example, Hult et al. (2005) and Morgan et al. (2009) have applied the information processing approach to studying marketing capabilities used for information generation and dissemination about customer value 
creation (Hult et al., 2005; Morgan et al, 2009). These market-oriented activities require individual cognitive structures which are involved in predicting and explaining of behaviour of others, in recognizing and remembering relationships between components and actors, and in constructing expectations of what is likely to occur next (Mathieu et al., 2000).

During business opportunity development, entrepreneurs develop cognitive structures not only for the interpretation of situations from a market-oriented perspective but also as the basis for market-oriented decision-making; decisions to engage in market-oriented behavior. In this study, we focus on cognition in terms of entrepreneurs' interpretation of opportunity development in a characteristically market-oriented way: Namely, focusing on the capture, creation and delivery of (superior) customer value. Since a market orientation is beneficial to the performance of start-ups, small and medium-sized businesses (Raju et al., 2011; Ghosh et al., 2001) entrepreneurs who develop a business opportunity profit from developing a market-oriented mindset and deploy market-oriented activities.

\section{MARKET-ORIENTED MENTAL MODELS}

The development of cognitive structures is characterized by the development of mental categories. Mental categories play an important role in providing a 'fit' between a situation and its meaning; those, in time, give rise to mental models (Autere \& Autio, 2000) which are interpretative frames used for understanding situations and events (Hill \& Levenhagen, 1995). Mental models are cognitive structures that represent "how the environment works" (Hill \& Levenhagen, 1995: p.1057). Mental models provide a means for individuals to create and share a more general (shared) understanding, for example by establishing images, names and other sensemaking processes of how things fit together (Hill \& Levenhagen, 1995).

While sensemaking processes are episodic and situational, they form the base for more stable mental models which establish how things fit together and what is important and unimportant depending on for example underlying values and common understandings (Hill \& Levenhagen, 1995). Mental models reflect individuals' cognitive frameworks that define plausibility, effectiveness, or some other form of acceptability (Pryor et al., 2015). In this study, we look at the emergence of market-oriented mental models which reflect sensemaking processes regarding problem solving for (superior) 
customer value creation. They play a critical role in enabling entrepreneurs to structure behavior in their organizations (Forbes, 1999).

The creation and maintenance of a market orientation requires the owner of a firm to have a mental model of his business which "points toward a culture with marketoriented values as the mechanism that enables the successful pursuit of opportunities for growth." (Martin et al., 2009: p.93).

The capture, creation and delivery of (superior) customer value during opportunity development requires interpretative processes because entrepreneurs must solve the problem of how to turn a yet non-existent business opportunity into superior customer value in the form of a service or product. Through every-day sensemaking processes entrepreneurs seek to successfully engage in that process.

\section{MENTAL MOdELS AND SENSEMAKING}

Studies have found that cognitive structures such as mental models are based on every-day sensemaking activities by entrepreneurs (Autere \& Autio, 2000; Hill \& Levenhagen, 1995; Krueger, 2007). Sensemaking processes begin "with attention, continues with encoding [...] [which] gives information meaning, by establishing a fit between the information and existing mental categories. As such categories develop, they become the source of mental frames, or mental models that are used in the individual sense-making process [and are] constructed in an ad hoc fashion to deal with novel problems and issues as they arise in the course of daily business operations" (Autere \& Autio, 2000: p. 8).

Sensemaking activities are associated with activities of attention - the perception of signals -, selection - in terms of the interpretation of meaning - and retention which involves internalization or storage of their interpretations (Pryor et al., 2015; Autere \& Autio, 2000; Weick, 1995) which underlines its close association to cognitive processes.

Sensemaking provides a frame of reference for cues, in order to the identify problems and the selection of an appropriate or desirable solution for them. It organizes the flux of impressions and perceptions which individuals are faced with in everyday life, by labeling what is considering noticeable (Weick et al., 2005). In that way, sensemaking processes facilitate our understanding of how things fit together (Hill \& Levenhagen, 1995). While sensemaking is said to be retrospective (Weick et al., 2005), it can also facilitate anticipatory forecast (Weick, 2012). The existing literature on entrepreneurial 
cognition has broadly acknowledged the analytical strength of the sensemaking perspective (Hill \& Levenhagen, 1995; Weick, 2005; Clarke \& Cornelissen, 2011). In order to examine what these cognitive processes of problem solving are, we undertake an inductive study among entrepreneurs in the phase of opportunity development.

\section{SENSEMAKING AS MARKET-ORIENTED PROBLEM SOLVING}

Sensemaking is used for the alignment of action towards a single purpose (Hitt \& Levenhagen, 1995), for example by noticing and labeling and thus categorizing events and phenomena (Weick et al., 2005). It forms the basis on which individuals create and share understanding, by establishing images, names or employing linguistic tools for the interpretation and meaning-making of their perceptions and intentions (Zott \& Huy, 2007; Weick et al., 2005).

Sensemaking is associated with solving issues or problems in a given situation because it is used to interpret and categorize 'chaotic reality' and stabilize the streaming of experience by creating 'plausible accounts of equivocal situations' and informing identity and action (Weick, 2012; Weick et al., 2005). By means of sensemaking, individuals strive to create legitimacy in their intentions, accounts and actions (Lounsbury \& Glynn, 2001; Wry et al., 2011).

Narver \& Slater (1990) posit that a market orientation "most effectively and efficiently creates the necessary behaviors for the creation of superior value for buyers and, thus, continuous superior performance for the business" (Narver \& Slater, 1990: p. 21). In this study, we focus on sensemaking processes that reflect how entrepreneurs solve problems they encounter during business opportunity development and how they develop a sense of legitimate, effective and/efficient solutions in their quest to capture, create and deliver (superior) customer value. For example, an entrepreneur who developed a food-processing technology interacts with a supplier and based on information exchanged in that interaction, learns that there is another potential applicability of his food-processing technology, for example in the dog food market. He realizes that instead of only approaching potential customers in his original market, he can also target relevant parties in the other market. The entrepreneur interprets a second potential product applicability as a way to more effectively create customer value, and decides to add a value offer for the second market with which he can satisfy the need of more customers (simultaneously) and potentially even satisfy their needs better. The entrepreneur's report of this situational, episodic sensemaking process reflects a more 
sustainable market-oriented mental model, namely that of communication - interaction with the supplier who provided the valuable information - as well as proactivity in the creation of (superior) customer value.

In graph 4-1 we have depicted the relationship between sensemaking processes, mental models and a market orientation. A market orientation consists of market-oriented mental models that reflect recurring solutions to problems which entrepreneurs encounter in the creation of (superior) customer value. Those mental models are rooted in individual, episodical sensemaking regarding problem solving as reported by entrepreneurs.

Graph 4-1: Conceptual model of the cognitive development of a market orientation

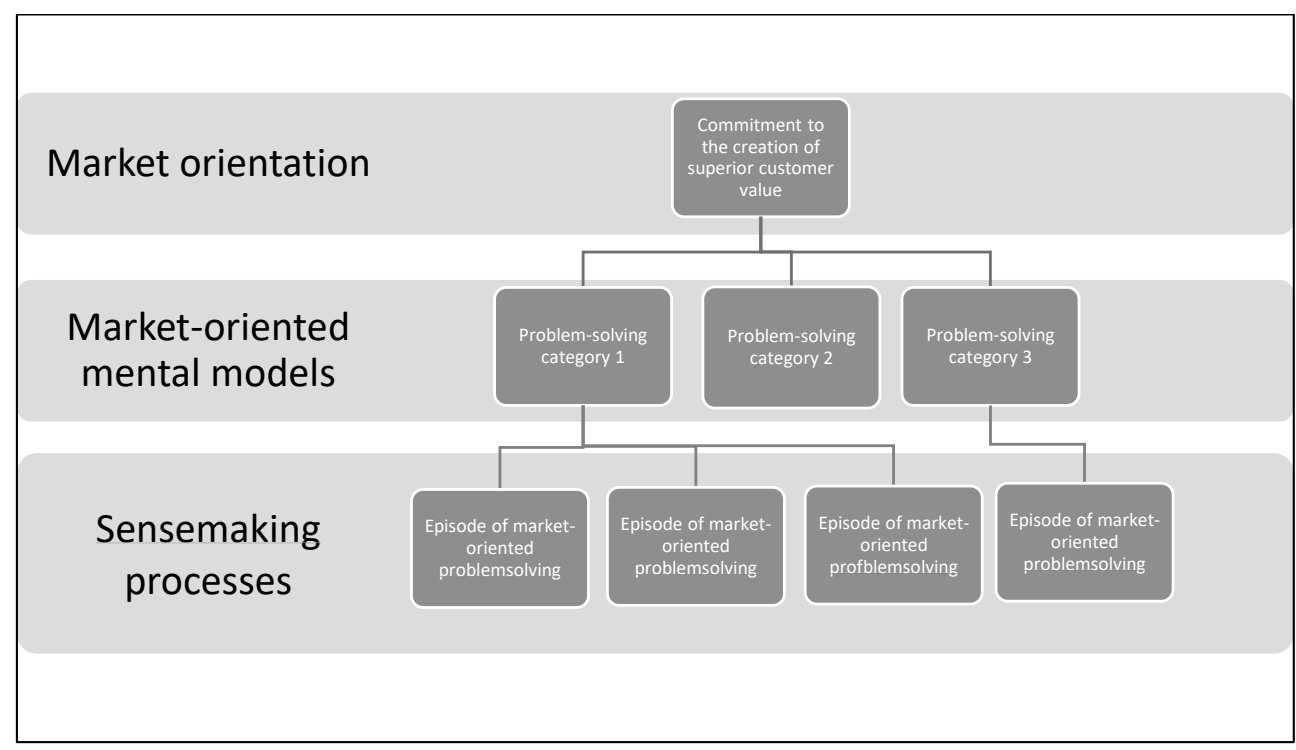

\section{ANALYZING SENSEMAKING PROCESSES}

Sensemaking processes can be analyzed using ethnographic methods. In ethnography, text and other ways of expression are studied in terms of how they represent reality since people as well as other social entities perceive and communicate reality differently. Silverman (2001) uses the poignant example of how the accounts of the 'same' event read differently depending on the newspaper they are written in.

Ethnographic methods reflect a concern with individual's sensemaking processes. Sensemaking is associated with labeling (Weick et al., 2005) which involves the categorization of new impressions. Sensemaking is associated with justification activities 
by actors (Weick, 2012). People identify and describe events in terms of many different categories, and those identifications of categories have "massive implications for the sense we attach to people and their behavior" (Silverman, 2001: p. 140). In particular, sensemaking involves categorization activities and the use of discursive tools.

\section{MARKET-ORIENTED PROBLEM SOLVING AS CATEGORIZATION}

We study people's representation of reality in terms of categorization (Silverman 2001) which is also central to sensemaking theory (Weick, 1995; 2012). In the case of this study, we focus on sensemaking processes related to market-oriented problem solving. Market-oriented problem solving involves the identification of a problem and a corresponding solution. A problem is an issue for which some kind of response is called (Spicker, 1995).

Problem solving consists of the response to a perceived problem, and therefore involves a process of labeling (Weick et al., 2005). This process is associated with 'functional deployment' which consists of the imposition of "labels on interdependent evets in ways that suggest plausible acts of managing, coordinating, and distributing" (Weick et al., 2005: p. 411). Through labeling, individuals differentiate and identify phenomena (Chia, 2000) - in this case, the solution to the problem of customer value capture, creation and delivery.

In this study we focus on the categorization of market orientated problem solving by entrepreneurs. This process involves labeling involves sensemaking in terms of plausible accounts of what problem related to the capture, creation and delivery of (superior) customer value was solved in what way. This translates to the entrepreneur's perception of a phenomenon encountered during the process of (superior) customer value capture, creation and delivery, and its categorization as a problem as well as the identification of a solution for it. For example, an entrepreneur realizes that he can better work together with a different distributor to get his product faster to the customer, the redesign of a product to make it more attractive to potential customers or optimizing internal processes to that end.

In order to discern sensemaking processes which involve that kind of marketoriented problem solving we draw on existing analytical concepts from the sensemaking literature, specifically the role of categorization and discursive devices. 


\section{THE USE OF DISCURSIVE TOOLS IN SENSEMAKING}

Plausible accounts of a certain event or phenomenon - like that of overcoming problems in (superior) customer value capture, creation and delivery - are associated with narratives. With narratives, entrepreneurs seek to establish identity and legitimacy of the business (Zott \& Huy, 2007; Clarke \& Cornelissen, 2011). Discursive devices are linguistic tools - such as metaphors, analogies, or narratives or even representative images - which are used in sensemaking in organizations (Hill \& Levenhagen, 1995; Stigliani \& Ravasi, 2012).

Discursive devices can be effectively used for making and conveying sense during entrepreneurial value capture, creation and delivery. Cornelissen \& Clarke (2011) point to the importance of linguistic tools for entrepreneurs during business opportunity development. For example, linguistic frames, labels and metaphors are used to convey the identity of an organization or company (Navis \& Glynn, 2010). In addition, entrepreneurs use images and other symbols in what has been described in 'symbolic impression management' during business opportunity development. (Zott \& Huy, 2007). The use of images involve sensemaking processes which instill those images with meaning (Stigliani \& Ravasi, 2012).

Therefore, when presenting the results of our analysis and where it is applicable, we describe the type of discursive element, or the association with entrepreneurial identity or legitimacy identified in the entrepreneur' reports of their problem solving involved in value capture, creation and delivery.

In sum, in this study we analyze the cognitive development of market orientation in terms of comparing mental models about customer value creation. Those mental models are studied by examining sensemaking processes about effective and/or efficient solutions to issues or problems during value creation. In the next section we describe the method used to carry out this study. 


\subsection{METHOD}

\section{RESEARCH DESIGN}

In this study, we examine how market-oriented problem solving yields mental models among entrepreneurs. To study this cognitive dimension of market orientation development we use a qualitative research design.

\section{QUALITATIVE RESEARCH}

A qualitative research design facilitates the analysis of evaluative, cognitive processes of individual actors (Rynes \& Gephart, 2004). Qualitative research is employed for the depiction and understanding of how meanings is given and (co-) constructed by individual actors (Rynes \& Gephart, 2004). It focuses on the socially constructed nature of reality (Schutz \& Luckmann, 1973) and is used in to identify how social experience and processes are given meaning (Denzin \& Lincoln, 2000; Rynes \& Gephart, 2004). A qualitative research design facilitates the identification of patterns from the data by comparing different cases in the sample of a study. By means of this crossexamination researchers derive conceptualizations and categorizations (Bingham \& Eisenhardt, 2011), in this case those which can contribute to theory-building of the development of a market orientation among entrepreneurs.

Since we seek to analyze the development of a market orientation in terms of sensemaking processes, we use an ethnographic approach to identify categories of market-oriented problem solving in the entrepreneurs' discourse. To do so, we use the grounded theory method.

\section{GROUNDED THEORY}

Grounded theory is an ethnographic method which enables theory development inductively, through the constant comparative method (Glaser \& Strauss, 1967). Grounded theory 'is a way of thinking about and conceptualizing of data' and its ontological roots can be found in the importance of discovery (Corbin \& Strauss, 1990; Strauss \& Corbin 1994). It is a qualitative research method based on the careful and systematic collection of data and its analysis, using the cross-comparison method in the analysis of empirical data with the aim of establishing conceptual relationships (Strauss \& Corbin, 1994). This method is used to compare units of analysis - in the form of text or discourse - which are coded into more abstract categories. In this way, one can 
examine similarities with and differences between items in different categories (Walsh, 1998).

The grounded theory approach examines which categories are used by individuals to label events and phenomena. The grounded theory method is characterized by a method of constant comparison of the data during the analysis (Walsh, 1998). In grounded theory, coding is seen as an important element of transforming raw data into theoretical constructions of social processes (Kendall, 1999). This leads to concept development with 'considerable meaningful variation' which is the base for theory development (Strauss \& Corbin, 1994), in this case about the development of a market orientation from a cognitive perspective.

This fits our study of the cognitive development of market orientation. We focus on categorization processes relating a market orientation, namely, how entrepreneurs report in what way they solve problems in capture, creation and delivery of customer value. To identify market-oriented problem solving among entrepreneurs we examine sensemaking processes of entrepreneurs involved in value capture, creation and delivery. We draw on data derived from entrepreneurs engaged in business opportunity development. Ultimately, we seek to contribute to theory building of the development of market orientation.

\section{RESEARCH SETTING}

The data comes from a population of entrepreneurs who participated in a business incubator/accelerator program. This VLT (Venture Lab Twente) program consists of a twelve-month long incubation/acceleration-trajectory for start-ups and small firms, coordinated the University of Twente, a technical university in the Netherlands. While the VLT program is geared towards the facilitation of high-growth firms, the majority of participating entrepreneurs are in phases associated with opportunity recognition, characteristically described in terms of discovery and formation of an opportunity (Lumpkin et al., 2004; Corbett, 2005).

The VLT program provides an intensive, weekly business acceleration program. It provides a variety of material resources - office space and facilities - to participants of the programs and offers immaterial resources, such as trainings, lectures and targeted feedback mechanisms in the form of mentoring and regular panel presentations. In addition, participants receive information about as well as introduction and access to appropriate (industrial) networks. Social sources of information, like those offered in the 
business incubation program, have a positive effect of entrepreneurial cognitive development (Ozgen \& Baron, 2007).

The incubation program is not only accessible to nascent entrepreneurs with no prior start-up experience, but also to more experienced entrepreneurs who want to develop a novel opportunity in addition to their existing business or to replace it. The VLT program therefore offers access to entrepreneurs with varying levels of prior entrepreneurial experience.

While the incubation/acceleration program's resources are aimed at facilitating the entrepreneur's venture's growth and commercial success, the incubation/acceleration program also provides a context inducing to the development of entrepreneurial cognition in general, by offering various learning instances. Of interest for this study is that the VLT program offers intensive interpersonal coaching and training, of which the trainers and coaches provide reference models to the entrepreneurs. Specifically, the VLT program trainings contain information about the importance of a value proposition, about defining one's market segment, how to develop an attractive opportunity; in short, it seeks to raise awareness for a market orientation among entrepreneurs. While this context creates a bias in terms of its favorable conditions for the development of a market orientation, those conditions also make it a suitable research setting to study this phenomenon because all respondents are involved in gestation activities which in turn invariably requires exposition to market-oriented problem solving for the creation, capture and delivery of (superior) customer value.

\section{DATA COLLECTION}

We collected the data by means of interviewing respondents from a population of entrepreneurs. These entrepreneurs are a) involved in novel business opportunity development and $b$ ) therefore likely to be engaged in market-oriented problem solving. By doing so we increase the chances that the sampled entrepreneurs report the development of market-oriented mental models.

\section{INTERVIEW TECHNIQUE}

The interview questions are not explicitly inquiring about the respondent's market orientation or the development of it. The reason for this is that we sought to avoid response biases related to 'socially desirable responding' (SDR). Social desirability is defined as evaluation apprehension or the respondent's tendency to respond in a "typical" fashion to avoid appearing "different" (Rosenberg, 1965; Shaver \& Scott, 1991). 
SDR can decrease significantly the validity of research results (Paulhus, 2002). Bird (2014) argues that the bias of SDR is a relevant issue when assessing entrepreneurs' thoughts, plans, decisions and actions. SDR occurs when entrepreneurs report seemingly desirable or deemed appropriate market-oriented attitudes, activities or learning. In order to avoid the bias of SDR in the respondents' answers we asked the respondents a variety of open-ended questions about their (novel) process of business opportunity development, their personal development as well as about their leverage and the perceived value of various resources offered by the business accelerator, such as trainings, coaching, panel presentations, office space offered as well as access to various networks.

The interviews consisted of a general query about the entrepreneur's own development as well as about his business opportunity. Asking open questions we queried about various aspects of opportunity development such as behavioral changes as well as intellectual or mindset development. Questions we asked the respondents include the following:

- Have you changed your approach of doing business during the last year and how?

- Have you changed your goals or your vision during the last year, and if so how and why?

- To what extent do you see yourself as an entrepreneur at this moment, and how is this different from a year ago when you started?

The complete list of interview questions can be found in Appendix 1. All interviews lasted between 40 minutes and 120 minutes. In order to ensure the validity and reliability of the collected data, all interviews were audio-taped and transcribed. The digital transcripts were transmitted to the software programs NVivo10 and Excel, where we also conducted the coding of the data and subsequent analysis of the quotes.

\section{SAMPLING}

We carried out structured, open-ended interviews with entrepreneurs who are engaged in business opportunity development at a university-based business incubation/acceleration facility in Enschede, the Netherlands. By drawing data from this sample we ensures that all entrepreneurs are involved in problem solving related to (superior) customer value capture, creation and delivery. Also, we sampled 
entrepreneurs with differing amount of entrepreneurial experience, different educational backgrounds and with a range of industrial sectors those entrepreneurs are part of.

In total, 65 entrepreneurs were interviewed within three months after their participation in the one-year incubation/acceleration program at the University of Twente during 2012 and 2014.

We discarded those respondents from our sample who are so-called intrapreneurs (working as employees in firms but enjoy a certain degree of autonomy) or family entrepreneurs (who work in an existing business for a family member). This led to an exclusion of ten entrepreneurs from our sample. Also, we did not take respondents into account who reported the abandoning of the business idea during the acceleration program or who had become employed during the program year. In this step, we discarded 5 entrepreneurs from our sample. In total, our final sample consists of 50 entrepreneurs.

\section{SAMPLE CHARACTERISTICS}

Our final sample contains 50 respondents of which only 4 are female. The average age of our sample population is 46 years, with a standard deviation of 9 years. On average respondents had 7 years of entrepreneurial experience.

In our sample we distinguished between novice entrepreneurs who are those with up to five years of entrepreneurial experience, and 'experienced entrepreneurs' who are entrepreneurs with more than 6 years of entrepreneurial experience. Experienced entrepreneurs are those who have earlier experience with setting up a venture. The definition of experience we operate is linked to the amount of time spent on gestation activities. Read \& Sarasvathy (2005) when looking at entrepreneurial expertise propose a rule-of-thumb of a minimum of ten years of deliberate practice for a novice (entrepreneur) to ascent to the rank of expert. However we also know that the 5-year survival rate of new ventures is less than $50 \%$ on average, and that ten years after starting up only $10 \%$ of the ventures remain in the market (Wise, 2013). This suggets that taking the 10 year benchmark would correlate our definition of experience predominantly to venture survival success, at the detriment of and ignoring the significance of entrepreneurial experience having being gathered by entrepreneurs throughout those ten years.

When looking at gestation time in order to assess when entrepreneurs are on average considered to have built up their venture, we see that the literature on 
entrepreneurship acknowledges that there is a highly skewed distribution of gestation times associated with venture development (Davidsson \& Gordon, 2009). Reported median and mean gestation times have been identified to lay between 32 and 76 months (Liao et al., 2005), the mean of which yields 4,5 years of gestation time associated with venture development. Rounded up this would mean that a five year period represents an average time frame in which entrepreneurs develop their venture. We take this benchmark of 5 years as a plausible and credible time frame in which entrepreneurs accumulate sufficient experience to be called 'experienced.' Distinguishing between novice and experienced entrepreneurs in that way, our sample of 50 respondents contains 26 novice entrepreneurs and 24 experienced entrepreneurs.

\section{CODING PROCESS}

The focus is on the cognitive dimension of a market orientation. The interviews we collected provided us with the discourse which we then searched for sensemaking processes associated with problem solving for the creation of superior customer value by entrepreneurs.

We coded the data in the qualitative software program NVIVO 10. With this program, part of text can be selected into nodes - categories - which help to identify frequently occurring topics in the textual discourse of the interview. By creating various nodes and coding quotes which we found to contain references to creating (superior) customer value.

Two coders read and coded the interviews independently of each other. The firstlevel coding consists of searching for discourse about the identification of problems and their solutions during the capture, creation and delivery of (superior) customer value, by means of behavior or mental activity.

Only quotes from the discourse are kept in the sample final sample which both coders agreed upon that they contain sensemaking which reflects market-oriented problem solving, namely those which relate to the creation of (superior) customer value. Those market orientated sensemaking quotes contain various sentences that represent logical chains of thought. Each of those sensemaking processes in the form of quotes represents a unit of analysis. In total, the coding yielded 157 distinctive units of analysis.

In a second round of coding we examined which mental models emerge from the different, episodical sensemaking processes we identified. To do so we categorized units of analysis inductively according to the theme they contain, for example the theme of 
collaboration. Quotes of entrepreneurs which contain references for the role of stakeholders such as suppliers and collaborative firms in the process of value creation were coded into the thematic category of collaboration. We noticed during coding that the entrepreneurs' discourse frequently contains sensemaking processes related to more than one theme. As a consequence, we allowed for multiple thematic coding of a unit of analysis.

From this second round of coding a number of thematic categories emerged. Those thematic categories contain sensemaking which reflects market-oriented problem solving. While the sensemaking processes pertain to idiosyncratic, episodic problem solving specific to the individual entrepreneur's situation, grouped together they reflect a more general theme in problem solving which emerges from the data. The theme reflects a more general mental model because it represents the common denominator shared by all sensemaking processes grouped in that category. 


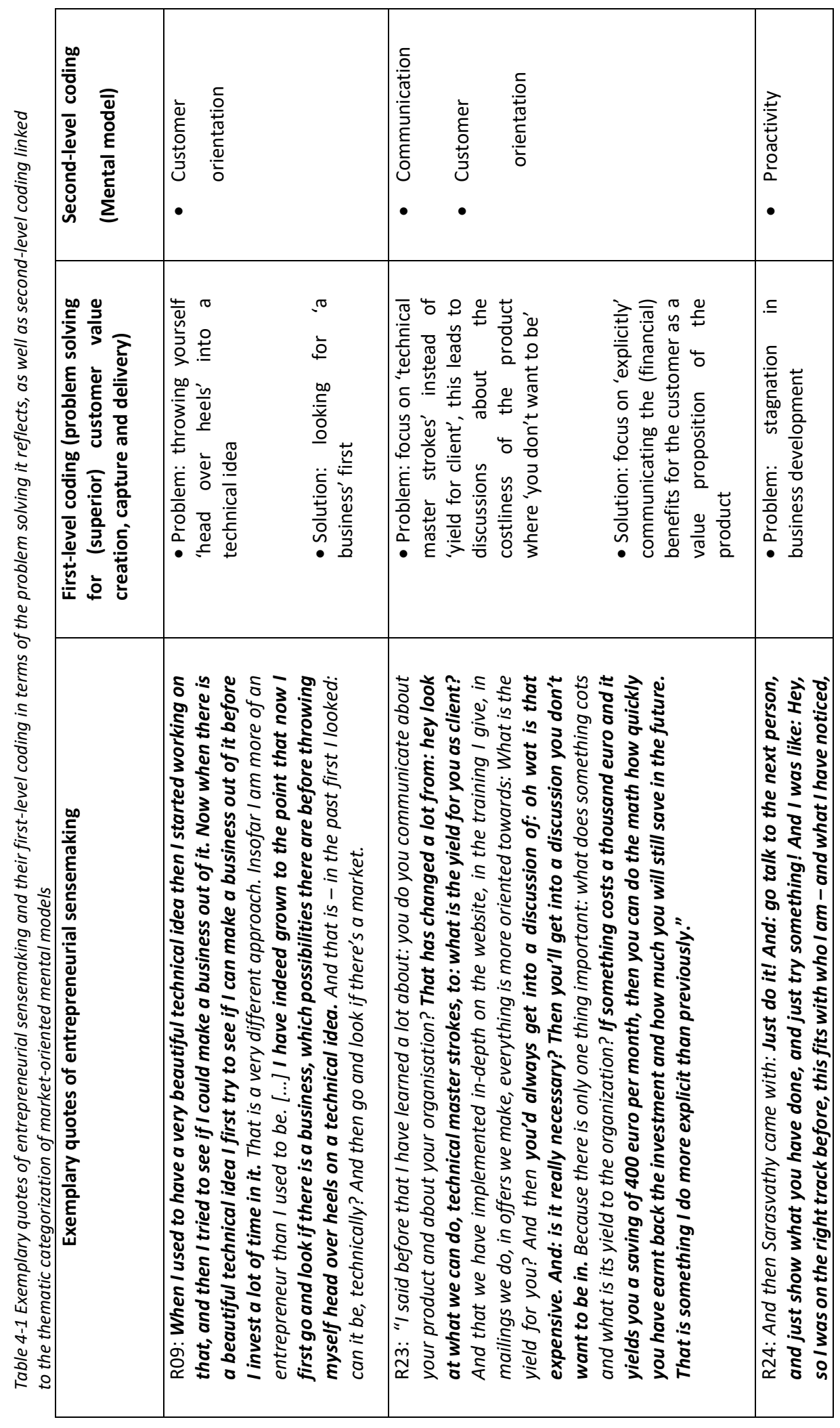




\begin{tabular}{|c|c|c|}
\hline 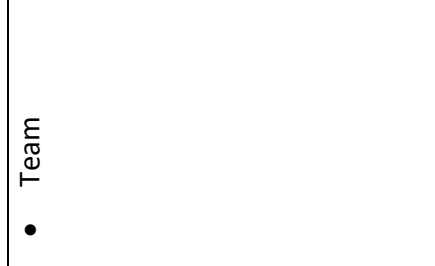 & 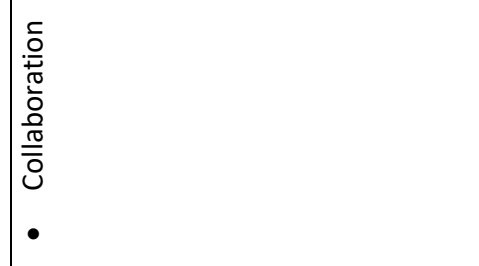 & 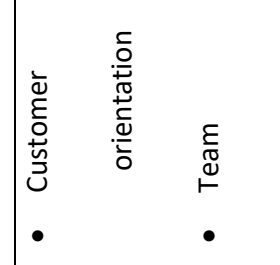 \\
\hline 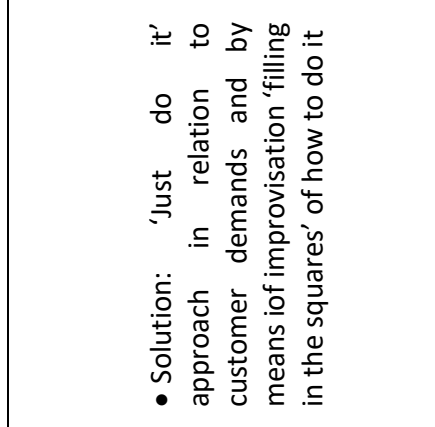 & 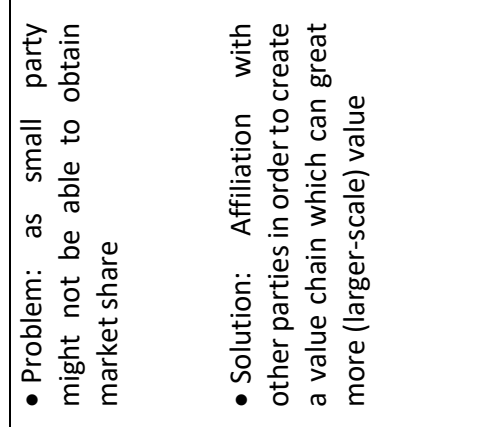 & 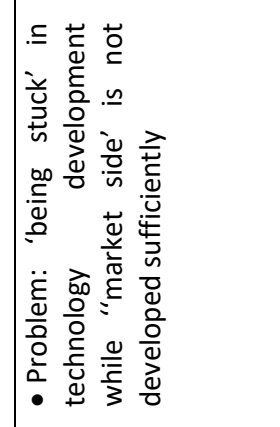 \\
\hline 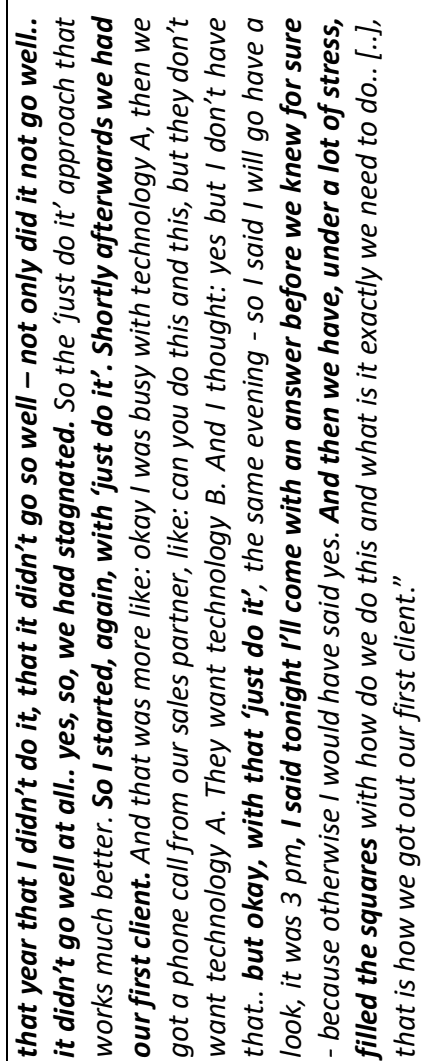 & 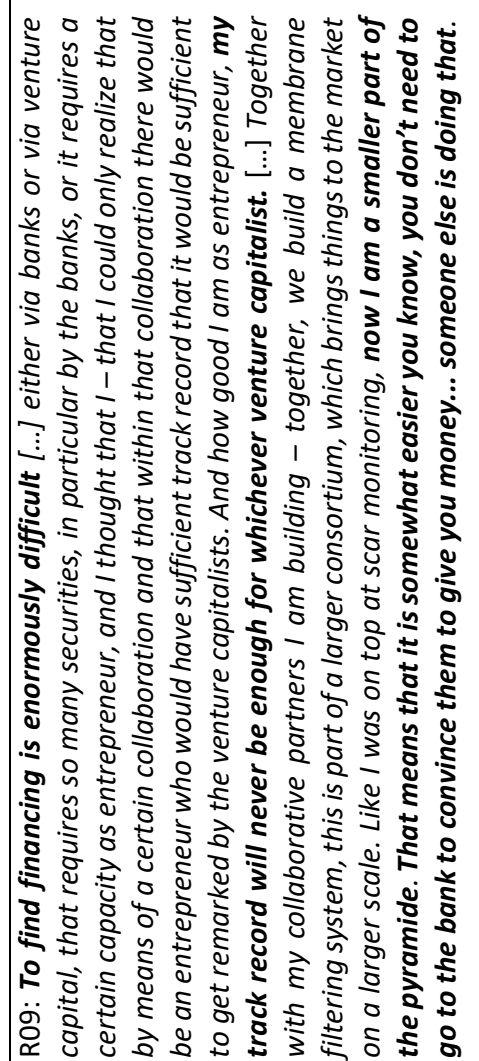 & 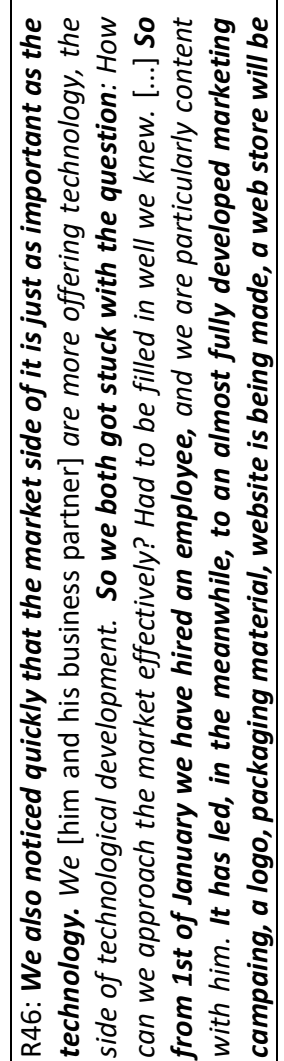 \\
\hline
\end{tabular}




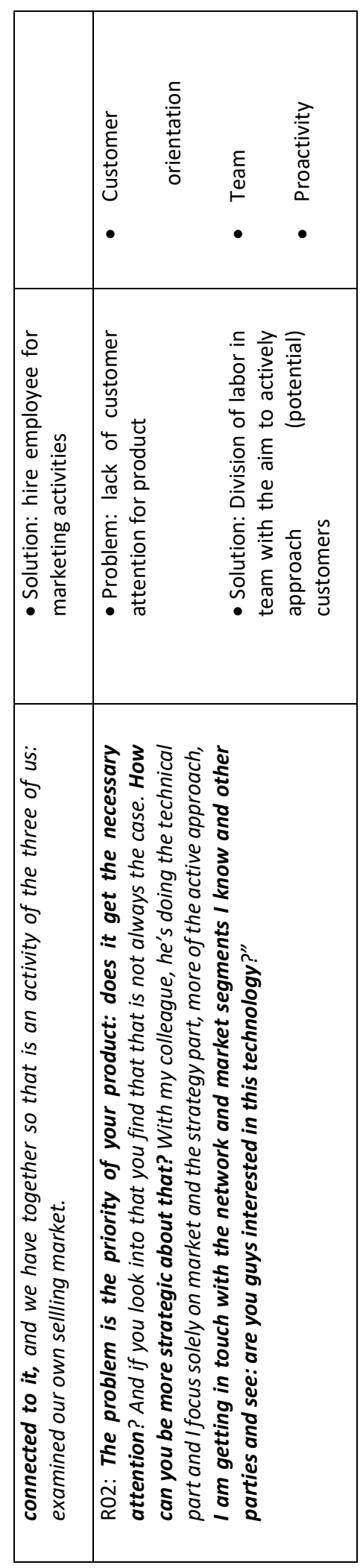




\subsection{RESULTS}

In this section we present the various problem solving themes we identified in the respondents' discourse. Also, we discuss how our findings relate to the existing concepts in market orientation literature. Then, we show how the different frequencies of reporting problem solving themes differs among novice and experienced entrepreneurs, reflecting the development of a market orientation.

\section{MARKET-ORIENTED MENTAL MODELS}

In total, we identified six distinctive categories of sensemaking processes which relate to market-oriented problem solving by respondents. Those six categories represent market-oriented mental models of which some are more frequently reported than others. Graph 4-2 contains an overview of the identified frequency of the mental models, in percentage of the total sensemaking processes related to problem solving for (superior) customer value creation. Below we describe sensemaking processes per mental model in more detail and provide some illustrative quotes to exemplify their use by respondents in our sample.

Graph 4-2 Frequency of thematic market-oriented mental models in percentage of all identified mental models in discourse

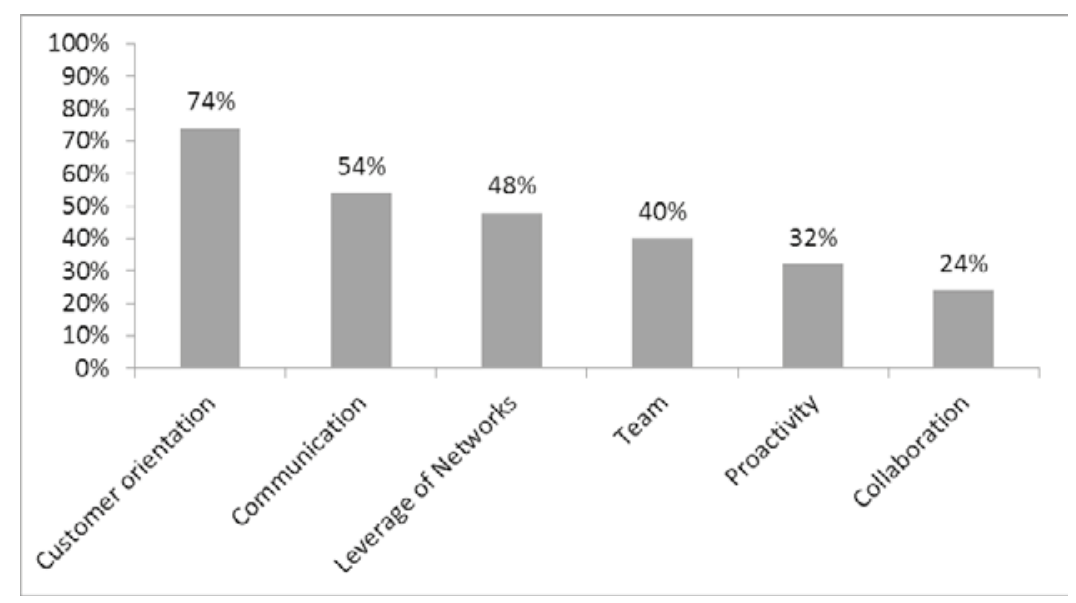

\section{CUSTOMER ORIENTATION}

The entrepreneurs in our sample most frequently report market-oriented problem solving that we labeled 'customer orientation'. $74 \%$ of all respondents report 
sensemaking processes which pertain to the centrality of the customer in solving problems related to the creation, capture and delivery of (superior) customer value. They describe problem solving by paying more attention to what value or benefits of the product or service offered by the entrepreneur actually matters for the customer. An example of it is given by the following respondent. He has learned that successful value creation is achieved by focusing his energy on the development of a specific product:

R31: "It is different now because a year ago I thought: let a thousand flowers bloom. Now I have to apply more focus.. I must get my own product to the market instead of waiting for what comes by. Now, I am focusing on developing a unique selling point.. at least obtaining my own position on the existing market"

This respondent describes how he used to think that value creation happens by means of spontaneous changes in opportunity development. The entrepreneur associates opportunity development with the freedom to try out whatever by describing it in terms of 'letting 1000 flowers bloom'. He evokes this image in the description of his own opportunity development process and then opposes it to the focus on a unique selling point which he is now concentrating on. He sees that focus as the solution in the problem of 'wild growth' of business ideas which he perceives as 'waiting for what comes by' and thereby being more active and goal-oriented which he associates with obtaining his own position on the market.

Another respondent reports a similar sensemaking process. Instead of focusing on the technical aspects which were important to himself, the entrepreneur has learned to orient himself pragmatically towards what is important to the customer in the development of his product. This change in attitude reflects the development of a customer orientation. The entrepreneurs reports how he's become aware of the importance of having a value proposition for the customer:

R10: "With our product there are so many use cases you can apply this product to. And the main challenge was to find the right direction - so again: the right focus - and how to sell the product. What's the pain - so what's the pain the product is supposed to solve? And really - focus on that [...] and then stay with this, stay with this use case, and don't move around too much... As soon as you have found the right niche, then the stay with this. That was quite - still like a challenge for me; I saw so many opportunities and it was hard for me to focus on one of them." 
This entrepreneur points out that the solving problems in creating value can be achieved by giving the customer an opportunity to express his wishes. By paying (more) attention at what the "pain" is for the customer the entrepreneur is able to find a "cure" for it, by doing so is able to create (more) value for the customer. The entrepreneur makes sense of problems in value creation by literally checking the customer for solutions - like a medical doctor who analyses a patient and asks him questions about his state of health before pronouncing the diagnosis.

These sensemaking episodes show how different linguistic tools such as images, symbols and metaphors are used by entrepreneurs for 'symbolic impression management' (Zott \& Huy, 2007) regarding their awareness of the importance of creating (superior) customer value. Both the '1000 flower' image and the 'pain-cure' metaphor used by these respondents exemplify how entrepreneurs emphasize an activation of or increase in their understanding of the role of the customer and his perception of the product or service.

Sensemaking processes in this category reflect the market-oriented mental model of 'Customer orientation'. This mental model relates to problem solving in terms of explicitly putting customer needs and wants central when solving problems of superior value creation.

\section{COMmunication}

Another category of sensemaking processes is associated with problem solving by means of communication. $52 \%$ of all respondents report the usefulness of talking with various stakeholders about one's business idea, in particular but not only with potential customers. Regarding the communication with potential clients, one entrepreneur remarks:

R02: "We ran into problems with translation of what we do in terms of relevance for the customer, communication must be more concrete. I have said on various occasions that I am busy with a tech platform and that there must be a translation of that in terms of solutions for the customer - that is a big problem. [...] You think that all is fine, but you must be able to say it very concretely."

This respondent describes the problem he perceives with creating customer value in terms of lacking communication with the customer about the concrete relevance of his product for the customer himself. The entrepreneur then describes the solution to this 
problem as translating the technology to solutions which are relevant for the customer. Another respondent solves this problem in the same way, as he describes in the following quote:

R23: "I thought that - I am a programmer but I always said I am not a nerd. [...] I always thought that I didn't communicate technically about our product, but that is what I did. I didn't realize that. And that is what I have learned. I have learned to look at it differently, more as if I was sitting in the customer' chair, like: hey, what is it doing for me? Instead of me saying: hey look at all what we can do."

The entrepreneur describes his change in terms of looking at it from a different perspective, the perspective of the customer. By drawing on the image of 'sitting in someone else's chair' he describes his learning but simultaneously, implicitly, states that the previous absence of taking the customer's perception was a problem. Having realized that he communicated 'technically' (as opposed to customer value-focused) he seeks to change that undesirable situation by communicating more effectively about his product. The solution of the problem is described by evoking the image of 'taking a seat' in the chair of the customer. Using this image, the respondent seeks to convey the meaning that he has made sense of the role of the customer who thinks 'what is it doing for me?' The chair is here used as a symbol for the increased awareness of the respondent that the perspective, perception, or interest of the customer is essential for successful value creation.

Respondents reported how they recognize that communication is not only about getting one's 'message' across but also to solving the problem of effectively obtaining relevant, valuable information for successful customer value creation. Even the non-oral cues matter, as another respondent has realized:

R38: "And also I learnt how to attend a network meeting. If there are people that you - you didn't know that how people are facing each other what that means, now I have learned that how they stand yields important information about how they are open to being approached and so on."

The respondent reports how he has learned the role of reading body language in successful networking. While learning about human interaction and thus (verbal and nonverbal) communication, entrepreneurs become skilled in how to communicate about their business idea in various ways and situations. By describing this learning, the 
entrepreneur indirectly admits that he was lacking this skill before and that his was an undesirable situation; after all he has changed it. The solution to this perceived problem is found in his learning about non-verbal communicative clues. The respondent's sensemaking reflects the perceived effectiveness of (non-verbal) communicative skill in network meetings that are attended by entrepreneurs with the aim of finding ways of creating superior customer value.

Sensemaking processes in this category reflect market-oriented problem solving by means of communication. Entrepreneurs demonstrate how they have realized the benefit of talking and obtaining or exchanging information with relevant stakeholders for customer value creation.

\section{LEVERAGE OF NETWORKS}

The category of sensemaking processes related to the leverage of networks is mentioned by almost half of all respondents, namely $48 \%$. Problem solving associated with these sensemaking episodes relate to solving problems by means of leveraging various networks, for example entrepreneurs' discourse about the importance of network building with strategic parties. For example, one respondent reports how he used to think differently about networks:

R30: What has also been a very important theme for me is - having insights in having and maintaining networks. About that I had - not a positive impression because I thought of it in terms of the traditional network meetings of business clubs and that sort of things, and the obligated drinking and casual chats. Here I learned, among other things, that you can look at it in a different way - that there you can instead set up a very professional network, that you don't necessarily need to maintain that network in a café environment but that you can do that in a very professional atmosphere. And if you really do your best and you maintain your network that there.. that that network - your network gives a lot, that you get very much back for it. What you have to look for in that network is a little bit - yes, that person who is engaged in that network in the same way, so who is not playing big boy but precisely those people who use that network to do business. And if you become aware of that a little bit, then - yes, then you design your network in order to do business. And, yes, I had a little bit of a chronic lack of that, of having and maintaining that network, and I found that here indeed." 
This respondent accounts for the problem with networking in terms of having relevant networks as well as maintaining them. He links networking to negative connotations; a 'café environment' which stands symbolic for an informal, unprofessional networking context. The entrepreneur then describes the solution to this problem. This he found to be in looking at networks as something that can be shaped by oneself, and therefore made to serve more professional leverage - namely "to do business". His problem of a chronic lack of networks and their maintenance he reports to solve by being selective and choosing carefully, that way safeguarding the quality - and usability - of his network and indirectly emphasizing the problem solving during (superior) customer value creation by means of the leverage of networks.

Another entrepreneur mentions the different functions which networks can fulfill when he reports his perception of networks in the following way:

R26: "Actually I don't do anything with social network. [..] What I - would find nice but that, until now it hasn't shown that it is in there - that is for example that as entrepreneurs you regularly, once in a while, get together and just exchange thoughts. That would be something - that is something I would like. [...] We are part of an entrepreneurs' club, I am part of the technology circle Twente, but that is - well that has proven to be much too technical, I have no affinity with that, technology doesn't interest me. So what it's for me about actually is more generally that you discuss, about this and that, about entrepreneurship, how you can bring ideas to the market, more about that sort of thing."

The respondent describes how he would like to leverage network more generally. He describes a problem which pertains to lack of a more general usability of his network. He report his affiliation with a 'technology'-oriented network. This stands as a metaphor for an orientation on product development and the technological component of it. This entrepreneur sees that as a problem, "too technical", and talks about the potential solution to this as laying in networks which can provide information about the ways of bringing ideas to the market. Here again, we see that networks are appreciated in their broad scope. The entrepreneurs identifies problem solving of too narrowly-defined, technology-oriented, networks as laying in those which can be leveraged to learn 'entrepreneurship' more generally, in terms of successfully delivering (superior) customer value. 
Market-oriented sensemaking in this category of problem solving relate to the role of networks in general. Networking sees as a solution in itself, In order to leverage that network for a variety of purposes related to the capture, creation and delivery of (superior) customer value. The sensemaking processes of respondents associated with problem solving by means of using the beneficial effects of networks, reflecting the mental model we named 'Leverage of Networks'.

\section{TEAM}

The problem solving theme of team is, as the name suggests, primarily rooted in the role of the team in realizing superior customer value. In the discourse of $40 \%$ of all respondents we identified reports of problem solving which involve the entrepreneur's team. One respondent for example points out the importance of organizational development in the creation of value:

R02: "You realize that you still have to do a lot. There is a whole period of organizational development and growth. Create value in a clever way in the organization; form a team; that's what it's also about, to do it together. By the way that is the biggest challenge in the short run: good team, finding good people."

The respondent realizes that there is still 'much more to do'. By emphasizing that it is 'also about doing it together' as well as the challenge of finding 'good' people because a 'good team' helps to 'create value in a clever way' because it is important to 'to it together'. This statement reflects market-oriented problem solving insofar as the entrepreneurs underlines the role of his team members in successful customer value creation.

Another example is given by a respondent who reminisces about the importance of doing customer-related activities. He reports how he perceived his own lack of commercial abilities as a problem and what he is sees as the solution to it:

R44: "Last year when I was starting with this business idea I was looking to commercialize this, and was looking for a partner who could provide services, so different stakeholders were mainly from people who could provide services. Why? Throughout the year I realized that commercial, or the sales part of the business, is also very important. And for this I need a Dutch partner, which means that I also have to sacrifice 
my independence a little bit and also has to help people to buy into this idea. In that sense the idea has changed."

The entrepreneur talks about the importance of having the sales person who can carry out sales activities. This indicates problem solving in value creation by means of functional division, namely separate marketing activities. While he recognizes it will limit his independence, he underlines that he needs a partner for the "sales side of the business". The reference to the delivery of customer value shows that for the entrepreneur, problem solving in (superior) customer value creation, capture and delivery can be achieved by the beneficial effect of a team. In this case, it concerns a team member who can take over the important task of selling the value proposition to potential customers.

Sensemaking processes in this category relate to team-based solutions problems in the creation, capture and deliver of value, and reflect the development of the mental model we called 'Team'. Entrepreneurs in our sample report how they start functional division of labor in order to better reach and serve a market, or more generally appreciate the help of team-members in market-related activities.

\section{Proactivity}

In the discourse of almost one in three respondents - $32 \%$ - we found reports of the role of proactivity in solving value creation problems. This sensemaking theme is linked to a proactive attitude towards business opportunity development, mainly in terms of customer acquisition:

R14: "Yes, in the sense that I do much more cold acquisition now. Before I had a, yes, an existing network which, say, provided one assignment after the other and that actually it wasn't necessary to do cold acquisition. End the last year I have actually for the first time = yes did I start doing that because actually it was necessary. Yes, by means of the business model - I don't know if you know that - well.. I have changed some things, in particular, say, more publicity, so actually also a little bit in order to support the cold acquisition- yes, that kind of thing."

The respondent reports how she had no interaction before with potential customers in the context of cold acquisition. But realizing that she had more problems getting assignments ("it was necessary") she has "changed some things" in terms of her marketing activities. The solution, for her, was to increase her efforts with cold 
acquisition, making publicity and displaying more behavior oriented versus proactive customer interaction. The solution for her problem of creation customer value is to engage more proactively with (potential) customer in order to better delivery value to them.

Another entrepreneur also sees problem solving in (superior) customer value creation, capture and delivery in terms of being proactive about solving issues. He reports tis in the following quote:

R43: "What I have learned most is that entrepreneurship and setting up a business is mainly just about doing it. For example, when you look at the market developmentso, how do you find clients who want to have your technology, who are interested eventually that is just a question of telling everywhere what you do. So, mainly networking, so, like: telling about what you do and at a certain moment the bal gets rolling. [...] So I don't know if you can talk about easy or difficult, it is just a question of just do it. And just being flexible in that - so I mean, actually, when there are things that are happening or that need to happen, yes, then you just have to deal with that and do it."

The respondent emphasizes the importance of just getting the things done which need to be done, for example by means of networking and telling people about the technology, thereby "getting the ball rolling". Here, communication is seen as a solution to the desired state of having clients ("how do you find clients") and the respondent reports a proactive, approach is seen as the answer to that problem. In that sense the entrepreneur accounts for problem solving in the case of finding clients who are interested in buying the product by means of proactivity. By pointing out that "this is really what we are going to focus on" this quote reflects his commitment to creating customer value by means of actively bringing his product to the market, by proactively engaging in marketing of his technology. The respondents discourse thus reflects problem solving by proactively engaging in interaction and communication to successfully create and deliver customer value.

This category of market-oriented problem solving contains sensemaking processes which relate to the entrepreneur's own behavior, namely his proactivity in approaching relevant market parties. This problem solving by means proactive engagement in customer value creation reflects a mental model we called 'Pro-activity'. 


\section{Collaboration}

A final category of sensemaking processes is related to problem solving in customer value creation by means of collaboration. We found sensemaking processes linked to this category of problem solving in the discourse of almost one in four respondents $(24 \%)$. This mental model reflects the importance of third parties in the creation of customer value.

For example, one entrepreneur has realized that he needs partners in order to realize the business opportunity he perceives. He says:

R05: "Based on the first suggestion we approached a number of companies where we know people. Went into a discussion, for two months, and from this a proposal emerged. Through discussing very much with each other and with whom we work together did we get it on paper. A demo-unit, that was the work of the team with the help of suppliers. By being the spider in the web eventually everything fell into place."

We see that the respondent reports both communication and collaboration in business opportunity development. In particular, this quote reflects the role of collaboration. He describes how 'eventually' customer value is facilitated - 'falls into place' -The entrepreneur indirectly defines the problem of insufficient (immaterial) resources by pointing out the solution through which everything 'falls into place'. He draws on the image of a spider in its web, thereby evoking the meaning of a resourceproviding mechanism which a web represents. By drawing on the image of a spider in a web, he thus emphasizes the role of collaborative partners in providing required (immaterial) resources for customer value creation, in this case for making a demo-unit.

Entrepreneurs learn that it helps to solve problems during customer value creation with collaborative partners, such as lead customers. For example one respondent talked about his business idea which has turned from an original product-idea or another product-idea which appears to be higher valued by customers at this point:

R43: "Those are actually two very big markets, where we need a certain time time to market. Well, that time to market, that is in our eyes - somewhat too long. That is why eventually we have started looking for: okay, can we have a product a little different, a derivative product with which we, say, on short term can turn a commercial turn-over? And that is [product B]. That is more a laboratory-cooling platform, for customers who are interested in plug and play, [..] so the customer really has interaction with the cooling platform, he's interested in that low temperature. Now, that idea - when 
I started there was only the idea of [product A] and [product B] has kind of been added for strategic reasons. So with the latter the focus of the business has shifted a little at the moment. But yes, it appears that ultimately - what happened during the last year is that now we are busy to market the [product B]. What we use their, for example is lead users."

This respondent perceives the time to market as 'too long', thus labeling it as a problem. He pursues to talk about how this was solved by changing the product to something which lead users found valuable enough to using it and, more importantly, buy it on a 'commercial basis'. Problem solving in the creation of customer value is achieved by this entrepreneur by means of collaboration with another party, in this case lead users who helped to develop his product by using it, and ultimately buy the product.

Sensemaking in this category relates to various stakeholders or parties with whom collaboration is sought or deemed useful in the context of successful opportunity development. These sensemaking processes reflect the market-oriented mental model we named 'Collaboration' because the solution to problems of customer value creation is found by means of cooperation, alliances or partnerships.

In this section, we analyzed the development of a market orientation in terms of the activation of mental models. To do so we examined sensemaking related to novel ways of problem solving among entrepreneurs. In the next section, we compare the mental models used by novice entrepreneurs and those used by experienced entrepreneurs.

\section{COMPARING MARKET-ORIENTED MENTAL MODELS OF NOVICE AND EXPERIENCED ENTREPRENEURS}

The central research question of this study pertains to the way in which a market orientation develops among entrepreneurs. In the previous section we analyzed the content of market-oriented problem solving in order to identify mental models that are used by entrepreneurs during business opportunity development. In this section, we compare the market-oriented mental models we identified in the discourse of novice entrepreneurs (with one to five years of experience) and of experienced entrepreneurs (with more than 5 years of experience).

Comparing the market-oriented mental models used by novice and experienced entrepreneurs yields insights into structural differences between them. These differences indicate a change in market-oriented mental models - and therefore a market orientation 
- when novice entrepreneurs transit to become more experienced entrepreneurs, which would indicate the development of a market-orientation among entrepreneurs. To identify potential structural differences between novice and experienced entrepreneurs, we examine two aspects of the market-oriented mental models: For one, the relative frequency - the number of occurrences - of market oriented mental models between the two groups of entrepreneurs. Second, we compare the content of market-oriented mental models between the two groups of entrepreneurs.

\section{FREQUENCY OF REPORTED SENSEMAKING}

In Graph 4-3 we see how market-oriented mental models identified among novice respectively experienced entrepreneurs compare to each other. The graph illustrates that experienced entrepreneurs in our sample report four of the six mental models more often than novice entrepreneurs.

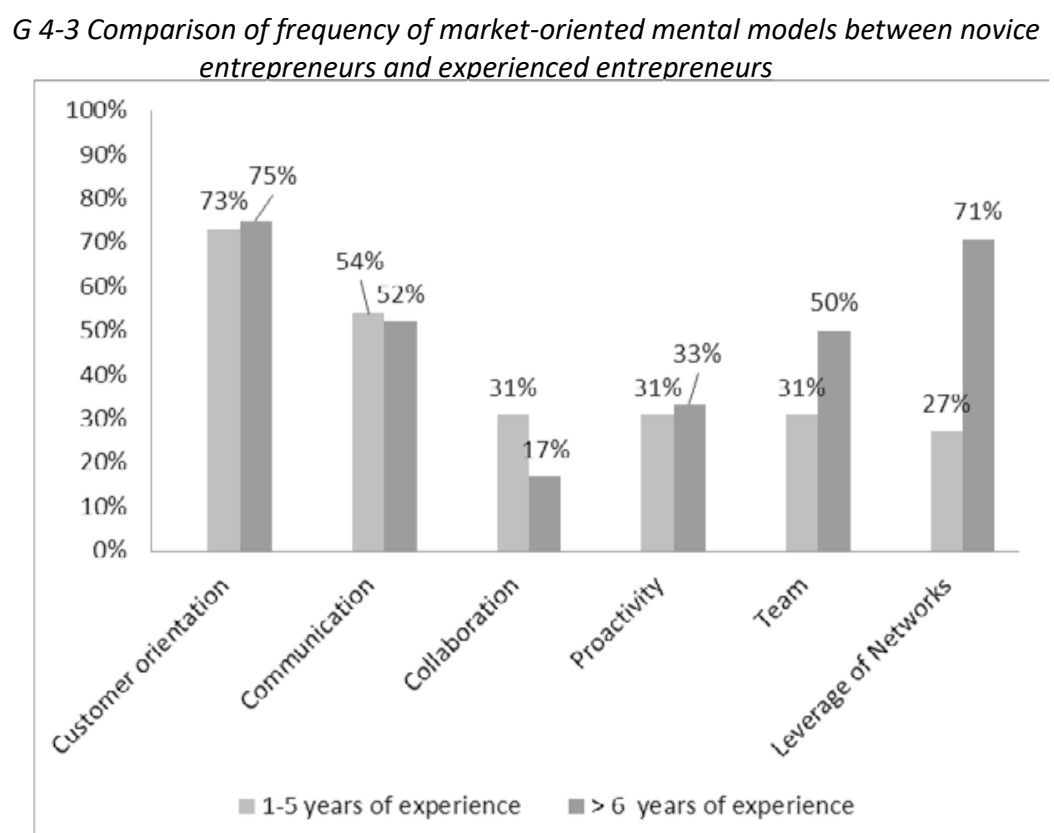

This shows that the activation of a market orientation - in terms of novel problem solving for the creation of customer value - is clearly not something only done by novice entrepreneurs, but even more so with increasing entrepreneurial experience. For example, an entrepreneur with more than 20 years of experience talks about her 
insecurities about not being sufficiently 'complete' in her abilities as entrepreneur and how this has changed due to the acceleration program she took part in:

R33: "For five years I didn't succeed in getting my discovery to the market, now, through the incubation program, I have become more 'complete', I have more selfconfidence, I can position myself better in the market, I can talk better to people who have a higher educational level than I have or with scientists."

This respondent describes that he didn't succeed in 'getting the discovery to the market', evoking the image of an existing market that he just could not reach yet. Then he enumerates a number of attributes and competences which have helped him to deal with this problem, in terms of more 'completeness', in terms of skills that help him to get to the evoked market. This quote reflects the entrepreneur's market-oriented problem solving in terms of his increased ability to verbalize his business idea towards other people, thus reflecting the mental model of 'communication' as a solution to his problem of 'getting the discovery to the market'. Another respondent, with more than 8 years of entrepreneurial experience, is developing a new business opportunity alongside his wellrunning small venture. He recognizes the following:

R23: "Now I look differently at customers, I have more focus on what the service or product can do for the customer."

This quote demonstrates that experienced entrepreneurs still learn to be market oriented. Regardless of the level of prior entrepreneurial experience, entrepreneurs report sensemaking processes which reflect the use of market-oriented mental models.

However, four of the six mental models we identified are relatively more mentioned by experienced entrepreneurs than by novice entrepreneurs. This suggests that a market orientation develops as entrepreneurs move from being novice to being more experienced, in terms of more frequently activated mental models linked to the creation of (superior) customer value.

\section{CONTENT OF MARKET-ORIENTED MENTAL MODELS}

In graph 4-3 we can see that the mental models labelled 'collaboration' and 'communication' are reported more by novice entrepreneurs than by experienced entrepreneurs, while 'Leverage of Networks' and 'Proactivity' are identified more in the discourse of experienced entrepreneurs. 
The mental models of 'Communication' and 'Collaboration' refer to concrete activities or engagement with particular stakeholders in problem solving. Mental models of 'Customer orientation', 'Leverage of Networks' and 'Proactivity' more generic and reflect more abstract solutions to problems in (superior) customer value creation. The more abstract content of the market-oriented mental models could refer to activities of communication and collaboration, but also to other activities linked to the creation of superior customer value creation. For example, the mental model of 'Customer orientation' could also be linked to problem solving by means of desk-research in order to gather information about customer needs by means of reading industry-related literature, and an example of problem solving by means of 'Leverage of networks' could be the attendance of a. industry-specific fair. In other words, those two mental models transcend concrete behavioral activities. This indicates that experienced entrepreneurs' discourse reflects mental models that involve a more general awareness of the value of networks in successful value creation.

The mental model of 'Team' is also more often mentioned by experienced entrepreneurs. But after checking the status of the business at the time of the interviews we saw that those entrepreneurs who report problem solving by means of a team, all but one have a team. It appears that sensemaking reflecting the mental model of 'Team' do so is associated with having a team to use for problem solving in the first place.

\subsection{CONCLUSION AND Discussion}

In this study, we studied the development of a market orientation among entrepreneurs. To do so, we examined sensemaking processes by entrepreneurs who are engaged in solving problems encountered in the creation of (superior) customer value. In total, we identified six market-oriented mental models reflected in entrepreneurial sensemaking which we labelled respectively 'Customer orientation', 'Communication', 'Leverage of networks', 'Team', 'Proactivity' and 'Collaboration'.

Below, we turn to a more in-depth discussion of these mental models identified and their linkage to existing market orientation theory. Then we discuss two major 
conclusions; first, how the central role of stakeholders reflects in market-oriented problem solving, and second, the way in which mental models used by more experienced entrepreneurs show characteristics of more abstract schemas which are also found at the organizational level.

\section{MARKET-ORIENTED MENTAL MODELS AND EXISTING MARKET ORIENTATION THEORY}

The six mental models identified show similarities to some main concepts of market orientation, from both the behavioral and the cultural perspective.

First, our results show that problem solving by means of a customer orientation is the most frequently reflected in the entrepreneurs' sensemaking. This is in line with the two major theoretical perspectives in the existing literature, which put an orientation towards respectively information acquisition, generation and dissemination about customer needs and wants central Narver \& Slater, 1990; Kohli \& Jaworski's, 1990). The development of the mental model of 'Customer orientation' consists of both ,responsive' (customer-led) and ,proactive" market orientation; the later is described as the satisfaction of latent needs (Narver et al, 2004). This mental model is also associated with problem solving regarding information generation activities described by Kohli \& Jaworski (1990) as an essential dimension of a market orientation. The predominance of the mental model 'Customer orientation' underlines the importance of a customer orientation in the cognitive conceptualization of a market orientation, in addition to the existing firm-level construct of the customer orientation described by Narver \& Slater (1990).

Also, we found that the mental model 'Team' parallels what has been described as inter-functional coordination (Narver \& Slater, 1990) respectively information dissemination (Kohli \& Jaworski, 1990). And the mental models of 'Communication' and 'Collaboration' compare to the concepts of information generation and responsiveness described by Kohli \& Jaworski (1990) because they imply communicative engagement with third parties in order to acquire relevant (customer or market) information.

The similarities described above between our findings and existing market orientation theory shows that there are a number of elements in our cognitive conceptualization of a market orientation which parallel existing theories, in particular the behavioral perspective - information generation, dissemination and responsiveness but also dimensions central to the cultural perspective on market orientation, namely customer orientation and inter-functional coordination - albeit inter-functional 
coordination not being equally important to customer orientation as it is conceptualized in the cultural perspective.

An important difference between the mental models we identified and existing literature relates to the competitor orientation (Narver \& Slater's, 1990). We could not identify sensemaking processes that pertain to problem solving associated with a competitor orientation in this study. Our results confirm those of Roersen et al. (2013) who found that entrepreneurs in small, startup ventures in Russia do not exhibit a competitor orientation.

The most crucial difference between our findings and existing theory is the central role that is accorded to stakeholders in those mental model we identified. While the mental model of 'Customer orientation' is the single most frequently mentioned mental model, our findings show that a market orientation primarily reflects in mental models related to the engagement of the broader network. In particular this is reflected in the mental models 'Communication', 'Leverage of Networks, 'Collaboration' and 'Proactivity' which pertain to interaction and go beyond a focus on customers.

Business studies widely recognize the significance of various stakeholders and networks for successful business opportunity development (Hite, 2005; Sullivan \& Marvel, 2011), which entrepreneurs leverage by 'tapping into' relevant (knowledgeable) stakeholders who can provide relevant market information and resources. These studies are exemplary for the effect of the so-called network economy (Achrol \& Kotler, 1999) in which entrepreneurs co-create, capture and deliver (superior) customer value together with other parties in their network. While customers are a focal point of value creation, the actual capture, creation and delivery of this value - which are recognized elements of the process of value creation (Anderson et al., 2009) - involve the leverage of relevant stakeholders (Shapiro \& Varian, 2013).

These findings link to the concept of socially situated cognition (SSC) described by Mitchell et al. (2011). SSC sees entrepreneurial cognition as embedded, distributed, action-oriented and embodied (Mitchell et al., 2011). For the context of our study, the focus of socially situated cognition on cognition-in-action and the role of stakeholders in shaping entrepreneurial development is particularly relevant. The market-oriented mental models we identified in this study are strongly related to learning about the importance of stakeholders in creating (superior) customer value. The cognitive development of a market orientation in terms of mental models thus reflects the important 
role that (proactive) engagement plays in problem solving during (superior) customer value creation. For example, entrepreneurs develop different cognitive strategies for market-oriented problem solving in terms of mental models of 'Communication' and 'Collaboration' which reflect not only an awareness of but an action-orientation (for engagement with the network) required for solving problems related to the creation of (superior) customer value. The results of this study thus provide empirical underpinnings for the concept of socially situated cognition and its relevance for analyzing and understanding the process of business opportunity development.

We conclude that market-oriented mental models function as socially situated cognitive mechanisms since they not only lead to an awareness for the social context that is relevant for solving problems in customer value creation, but also to engagement with networks in order to solve those problems.

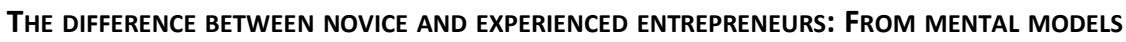
TO MENTAL SCHEMAS

Graph 4-3 shows that experienced entrepreneurs more often report sensemaking processes that reflect market oriented mental models than novice entrepreneurs. In addition, there are also differences in the content of market-oriented mental models used by novice and experienced entrepreneurs.

Market-oriented mental models used by novice entrepreneurs are associated with clear and tangible solutions to problems encountered during the creation of (superior) customer value, specifically the mental models 'Communication' and 'Collaboration'. Meanwhile, experienced entrepreneurs use mental models that link to more general solutions to problems related to customer value creation, such as 'Customer orientation' and 'Leverage of networks'. These mental models are more abstract than those used by novice entrepreneurs.

This difference in market-oriented mental model used by novice and experienced entrepreneurs suggests that a market orientation transforms with increasing entrepreneurial experience from concrete behavioral to more abstract and schematic market-oriented problem solving. Schemas are generalized set of rules in relation to classes of goals (Cheng \& Holyoak, 1985) and represent generic concepts stored in memory (Brewer \& Nakamura, 1985). They are more abstract and tacit than mental models (Sims \& Gioia, 1986; Orlikowski \& Gash, 1994), giving meaning to every-day 
action and the appropriate behavior or sequence of events in situations (Gioia \& Poole, 1984; Rerup \& Feldman, 2011).

The experienced entrepreneurs' development of more generic mental schemas can be explained by their level of expertise. Dreyfus \& Dreyfus (2005) examine the development of expertise and found that skill acquisition occurs in five stages of performance. The highest stage of skilled performance is that of expertise. While lower levels of skilled performance require conscious decision-making, the highest level of skilled performance - expertise - is based on the making of intuitive, immediate, unreflective situational responses which are more tacit in nature. The development of mental schemas by experienced entrepreneurs is linked to their acquisition of expertise. Both schemas and expertise are rooted in skilled performance, i.e. performance which reflects the appropriate behavior legitimately considered 'skilled' and both are characteristically tacit in nature.

We conclude that the use of more abstract schemas for market-oriented problem solving by experienced entrepreneurs can be explained by their more expertly and intuitive - tacit - performance in the creation of (superior) customer value. And the lower stage of performance by novice entrepreneurs explains their use of mental models that pertain to their concrete and activity-related decisions in market-oriented problem solving performance.

Dreyfus \& Dreyfus (2005) observe that experts, when asked to talk about their expertise, draw on 'deliberative rationality' which is detached, reasoned observation of the expert's own intuitive, practice-based behavior. This is opposed to what Dreyfus \& Dreyfus (2005) call 'calculative rationality', the inferential reasoning observed in the discourse of novice entrepreneurs and reflected in their use of more explicit mental models such as 'Communication' and 'Collaboration'. In order to develop expertise, Dreyfus \& Dreyfus (2005) note that "the beginner's job is to follow the rules and gain experience" (Dreyfus \& Dreyfus, 2005: p. 786). The authors argue that if the learner watches someone good at doing something this could limit the learner's random trials to the more promising ones. Therefore, observation and imitation of the activity of an expert can replace a random search for better ways to act. They conclude their argument by saying that this is, in general, the advantage of being an apprentice. 
Considering these insights in the light of the differences in market oriented development we propose the following. As we saw in the results, novice entrepreneurs use mental models which are clear in what skilled performance entails; decision-making regards reflective choices for explicit, concrete behaviors such as communication and collaboration. On the expert level of experienced entrepreneurs, decision-making about market-oriented problem-solving is made on a more intuitive and unreflective level and reflect in market-oriented mental schemas.

Mental schemas are broad and general enough to be a shared frame of reference from which concrete behaviors are drawn (Vaghely \& Julien, 2010; Rerup \& Feldman, 2011). Entrepreneurs must be able to communicate those schemas to new members of the firm. Especially for more experienced yet growth oriented entrepreneurs this is a point of attention. To communicate a market-oriented frame of reference to new members the expert would need to 'go back' into a lower, more reflective stage of decision-making of skilled performance because then he can make his own performance more reflective and more explicit and communicate this easier to other members of the organization.

Entrepreneurs, by developing their awareness of their level of skilled performance, can engage intentionally and effectively with new members of their organization. In particular, experienced entrepreneurs might consider that novice members of the firm will most likely be learning by the application of 'calculative rationality' as described by Dreyfus \& Dreyfus (2005). This means they will benefit from imitation and observation of 'how things are done', which also applies to learning about market-oriented behavior and attitudes.

In sum, we conclude the market-oriented mental models used by experienced entrepreneurs reflect more abstract problem solving than the mental models used by novice entrepreneurs. This difference between novice and experienced entrepreneurs parallels the difference in experience and skilled performance between beginners and experts as described by Dreyfus \& Dreyfus (2005) which affects the way entrepreneurs can transmit their market-oriented attitudes and behaviors to new members of their venture. 


\subsection{CONTRIBUTION}

A market orientation contributes to the optimization of a venture's performance (Bhuian et al., 2005). As yet, we lack analytical tools to examine how a market orientation develops and is maintained at the individual level (Gainer \& Padanyi, 2005). The existing measures lack applicability in the case of smaller businesses (Raju et al., 2011) which includes start-up ventures or self-employed entrepreneurs. With our study on the development of a market orientation among individual entrepreneurs we seek to fill this void and contribute to theory and practice in the following ways.

First, by conceptualizing cognitive development of a market orientation in terms of six mental models this study adds to our understanding of the quality of a market orientation among individuals called for in the literature (Morgan et al., 2009). Grégoire et al. (2011) encourage the study of development of entrepreneurial cognition. In this study we answer this call and focus on the cognitive development of a market orientation among entrepreneurs. With the results of this study we add to a better understanding of micro-level roots of a venture's market orientation. In that way we answer a call for conceptualization of micro-level processes associated with value creation (Kraaijenbrink et al., 2010). In table 4-2 we compare the traditional conceptualization of a market orientation and the market-oriented mental models we identified in our analysis.

Table 4-2 The market orientation construct as conceptualized by Narver \& Slater (1990) and Kohli \& Jaworski (1990) and corresponding mental models identified in this study

\begin{tabular}{|c|c|c|}
\hline $\begin{array}{c}\text { Concepts in existing literature on market } \\
\text { orientation }\end{array}$ & $\begin{array}{c}\text { Corresponding market oriented mental } \\
\text { model }\end{array}$ \\
\hline $\begin{array}{c}\text { Customer orientation } \\
\text { (Narver \& Slater, 1990) }\end{array}$ & $\bullet$ & $\begin{array}{l}\text { Customer orientation } \\
\text { Proactivity }\end{array}$ \\
\hline $\begin{array}{c}\text { Competitor orientation } \\
\text { (Narver \& Slater, 1990) }\end{array}$ & No corresponding market-oriented \\
\hline Inter-functional coordination & mental model identified \\
\hline (Narver \& Slater, 1990) & $\bullet$ & Team \\
\hline Information generation & $\bullet$ & Leverage of Networks \\
\hline (Kohli \& Jaworski, 1990) & $\bullet$ & Communication \\
& $\bullet$ & Leverage of Networks \\
& $\bullet$ & Collaboration \\
\hline Information dissemination & $\bullet$ & Proactivity \\
\hline (Kohli \& Jaworski, 1990) & $\bullet$ & Team \\
\hline Responsiveness & $\bullet$ & Leverage of Networks \\
\hline
\end{tabular}




\begin{tabular}{|l|ll|}
\hline (Kohli \& Jaworski, 1990) & $\bullet$ & $\begin{array}{l}\text { Leverage of Networks } \\
\text { Collaboration }\end{array}$ \\
\hline
\end{tabular}

The mental models we identified in our analysis parallel some dimensions identified in the existing literature, however there are two crucial differences between our findings and existing theory. One is the lack of mental models which pertain to the development of a competitor orientation. The other is the central role that is accorded to stakeholders in the market-oriented mental models we identified, in the form of the mental models 'Leverage of Networks', 'Communication' and 'Collaboration'.

Second, our findings show how mental models function as socially situated cognitive mechanisms for involving relevant stakeholders in solving problems that the entrepreneur encounters during the creation of value). Various authors have described the intersubjective, socially embedded nature of business opportunities (Davidsson, 2001; Venkataram et al., 2012; Mitchell et al., 2011; Clarke \& Cornelissen, 2011). This study contain empirical evidence of the socially situated and embedded nature of entrepreneurial cognition. The importance of a stakeholder orientation among entrepreneurs is for example confirmed by Whalen \& Akaka (2016). Innovative entrepreneurs must orient themselves towards relevant stakeholders and therefore this stakeholder-orientation becomes an important part of their market orientation.

Third and more practically, insights from this study can be used to facilitate venture development in business support programs. Support programs can profit from the results of this study by facilitating the development of market orientation mental models, not only among novice but also among experienced entrepreneurs. In addition, business support institutions can use the results of this study to find ways to foster a market orientation among entrepreneurs more holistically. For example, as we saw, entrepreneurs neglect the element of interfunctional coordination and virtually ignore the element of competitor orientation, even those entrepreneurs with numerous years of prior start-up experience. The raising of awareness for these elements in terms of trainings can help to make entrepreneurs more market-oriented, and subsequently more have a positive effect on the venture's performance in terms of profitability.

Importantly, the insights in differences between novice and experienced entrepreneur regarding their level of skilled performance reflected in their use of market oriented mental models has implications for the development of a market orientation of 
the organizational level, specifically when considering the growth of a market-oriented firm culture among all members of the organization. Entrepreneurs could grow their awareness of their level of skilled performance. In this, it is important that experienced entrepreneurs remain aware that novice members of the firm will most likely be learning by imitation and observation (Dreyfus \& Dreyfus, 2005) whereas novice entrepreneurs are more likely to be able to leverage discursive 'calculative rationality' with novice members since they are more reflective and less intuitive than experienced entrepreneurs about their entrepreneurial performance. By being aware of differences in their expertise and how to transmit knowledge about the 'right' way of performing to new firm members, both novice and experienced entrepreneurs can make their marketoriented attitudes and behaviors easier accessible to new members of their organisation.

\subsection{LIMITATIONS AND FUTURE RESEARCH}

There are three limitations to this study, as well as suggestions for future research it yields.

One limitation of this study is associated with the research setting. Our data stems from a sample of entrepreneurs which is drawn from a research incubation/acceleration program. This means that our sample is self-selectively biased, consisting of entrepreneurs who chose to actively leverage institutionally-set resources for the development of their business opportunity. This setting yields a sample-bias of entrepreneurs unwilling or able to independently pursue business development but clever enough to seek help. Therefore we cannot compare our findings with those of a sample of entrepreneurs who 'independently' pursue the realization of their business opportunity outside the resource-rich environment of the incubation program. Due to the setting of this study in an institutional support context makes that conclusion of this study are not necessarily generalizable for all entrepreneurs. However, our sample consists of a diverse selection of educational and industrial backgrounds. In that way we sought to validate the results across a wide variety of entrepreneurs and increase the reliability of our findings.

Secondly, we cannot exclude the possibility of a biased research population. The VLT incubation/acceleration program clearly favors the activation of a customer 
orientation, due to the program's emphasis on customer value creation, as can be seen in various trainings in which the development of customer marketing and sales skills of entrepreneurs are central. In general, entrepreneurs in the incubation/acceleration program are positively biased towards being market oriented to start off with; after all, their engagement in that program testifies of their commitment to the creation of (superior) customer value which is characteristic of a market orientation. This positive bias means that the entrepreneurs' readiness to develop their market orientation is generally higher in the totality of our sample. Yet, we see that a market orientation among entrepreneurs does not develop in accordance with the existing market orientation concepts. Even if the totality of the sample is more inclined to and trained in developing their market orientation by going through the business incubation trajectory with trainings and coaching on market orientation, this makes our results with ever more so relevant, in particular the development of a stakeholder orientation which is much more predominant in the market-oriented mental models we identified than in the market orientation construct advanced by Narver \& Slater (1990) respectively Kohli \& Jaworski (1990).

The incubator setting, in that sense, provides what is described by Shepherd \& Patzelt's (2017) as a 'community of inquiry' which consists of stakeholders who are able to comment on the potential opportunity's promise and validity. Those communities are important because by means of social interaction with others a community of inquiry exposes the entrepreneur to alternative thought worlds, give attention to his statements, and provide him with feedback and valuable information only obtained (Autio et al., 2013). Our findings demonstrate that it is important for entrepreneurs to be immersed and engaged in market-oriented activities offered in an incubator setting since this is conducive to change in terms of market orientation development - both among novice and experienced entrepreneurs.

A third limitation is due to the qualitative nature of this study which characteristically yields a sample size which is rather limited and therefore inhibit broader conclusions. Also, due to the small sample size we were unable to control for the effects of other variables which could affect market orientation development, such as differences in gender, educational background or team composition on the development of a market orientation in more depth. The ground work done in this study will need to be investigated further by taking personal attributes and contextual variables into account. 
In terms of avenues for future research we discerned three topics worth investigating in following studies.

For one, there is merit in gaining a better understanding of micro-level processes in venture development. The approach taken in this study differs significantly from that of Narver \& Slater (1990) or Kohli \& Jaworski (1990) yet support findings of more recent studies of the importance of stakeholders (Whalen \& Akaka, 2016). Further insights into the stakeholder orientation are therefore relevant for our understanding of a market orientation in small and medium-sized businesses. Therefore we propose that future studies can contribute by examining the effect of a stakeholder orientation - in the form of stakeholder-oriented mental models - on small and medium-sized venture performance, survival and growth. On a related note, it is relevant to examine how other variables enhance or prevent the development of a stakeholder orientation among entrepreneurs; for example; how do gender, age, or the type of industry which is targeted affect the development and use of stakeholder-oriented mental models we identified? We propose that future studies on those variables would yield relevant insights into the correlation of stakeholder-oriented mental models to venture success.

Second, the cognitive development of a market orientation in terms of mental models reflect the important role that (proactive) engagement of relevant stakeholders plays in problem solving during (superior) customer value creation. Our findings link to the concept of socially situated cognition described by Mitchell et al. (2011), therefore a future alley of research is the investigation of other mental models as socially situated cognitive mechanisms which enable entrepreneurs to understand and act in a more market-oriented fashion. For example, do entrepreneurs use certain mental models in other social contexts which affect their own cognitive development, such as when choosing team members, business coaches, or most trusted advisors relevant for mediated sensemaking (Strike \& Rerup, 2016)?

A third aspect worth future investigation is that of more generic mental models which we found among experienced entrepreneurs. These generic mental models have characteristic of schemas, in the sense that they are more abstract than situation-specific and can thus be applied in varying contexts. In that sense, they are a suitable base for the emergence of organizational schemas which ensure that individual efforts of everyday business activities are linked to the achievement of common organizational goals, by informing individual-level mental models and thus mainstreaming their idiosyncratic 
efforts towards a collective goal. It would be worthwhile examining the mechanisms by which individual mental models become firm-level schemas leveraged for the concerted creation of superior customer value.

Fourth, we suggest investigating the role of business acceleration programs in more depth. We found that the resources of the business support program had a considerable influence on the development of a market orientation among the entrepreneurs in the study sample. Market orientation development is stimulated by feedback not only from team members, suppliers, or other entrepreneurs but also from the business acceleration program that the respondents participated in. This strongly points towards the benefits of targeted feedback such as coaching but also trainings or organized access to networks. Encouragement, support and critical feedback on one's actions during business opportunity development appear to have an important influence on the cognitive development of entrepreneurs. The role of feedback mechanisms in business accelerator/incubator programs therefore deserves more attention in future studies.

\subsection{BIBLIOGRAPHY}

Achrol, Ravi S., and Philip Kotler. "Marketing in the network economy." The Journal of Marketing (1999): 146-163.

Anderson, J. C., Narus, J. A., \& Narayandas, D. (2009). Business market management: Understanding, creating, and delivering value.

Autere, J., \& Autio, A. (2000). Is entrepreneurship learned? Influence of mental models on growth motivation, strategy, and growth. In Academy of Management Conference.

Baron, R. A. (2004). The cognitive perspective: a valuable tool for answering entrepreneurship's basic "why" questions. Journal of business venturing, 19(2), 221-239.

Becker, J., \& Homburg, C. (1999). Market-oriented management: a systems-based perspective. Journal of Market-Focused Management, 4(1), 17-41.

Bhuian, S. N., Menguc, B., \& Bell, S. J. (2005). Just entrepreneurial enough: the moderating effect of entrepreneurship on the relationship between market orientation and performance. Journal of business research, 58(1), 9-17. 
Bingham, C. B., \& Eisenhardt, K. M. (2011). Rational heuristics: the 'simple rules' that strategists learn from process experience. Strategic Management Journal, 32(13), 14371464.

Bird, B. (2014). Toward a taxonomy of entrepreneurs' behavior. Handbook of Entrepreneurial Cognition, 113-131.

Blank, S. (2013). The four steps to the epiphany. Pescadero: K\&S Ranch.

Boso, N., Story, V. M., \& Cadogan, J. W. (2013). Entrepreneurial orientation, market orientation, network ties, and performance: Study of entrepreneurial firms in a developing economy. Journal of Business Venturing, 28(6), 708-727.

Brewer, W. F., \& Nakamura, G. V. (1984). The nature and functions of schemas. Center for the Study of Reading Technical Report; no. 325.

Cheng, P. W., \& Holyoak, K. J. (1985). Pragmatic reasoning schemas. Cognitive psychology, 17(4), 391-416.

Chia, R. 2000. Discourse analysis as organizational analysis. Organization, 7(3): 513518

Clarke, J., \& Cornelissen, J. (2011). Language, communication, and socially situated cognition in entrepreneurship. Academy of Management Review, 36(4), 776-778.

Corbett, A. C. (2005). Experiential learning within the process of opportunity identification and exploitation. Entrepreneurship Theory and Practice, 29(4), 473-491.

Corbin, J. M., \& Strauss, A. (1990). Grounded theory research: Procedures, canons, and evaluative criteria. Qualitative sociology, 13(1), 3-21.

Davidson, D. (2001). Subjective, intersubjective, objective (Vol. 3). Oxford University Press.

Davidsson, P., \& Gordon, S. R. (2009). Nascent entrepreneur (ship) research: A review. Denzin, N. K., \& Lincoln, Y. S. (2000). The discipline and practice of qualitative research. Handbook of qualitative research, 2, 1-28.

Dreyfus, H. L., \& Dreyfus, S. E. (2005). Peripheral vision expertise in real world contexts. Organization studies, 26(5), 779-792. 
Forbes, D. P. (1999). Cognitive approaches to new venture creation. International Journal of Management Reviews, 1(4), 415-439.

Gainer, B., \& Padanyi, P. (2005). The relationship between market-oriented activities and market-oriented culture: implications for the development of market orientation in nonprofit service organizations. Journal of business research, 58(6), 854-862.

Ghosh, B. C., Liang, T. W., Meng, T. T., \& Chan, B. (2001). The key success factors, distinctive capabilities, and strategic thrusts of top SMEs in Singapore. Journal of Business Research, 51(3), 209-221.

Gioia, D. A., \& Poole, P. P. (1984). Scripts in organizational behavior. Academy of management review, 9(3), 449-459.

Glaser, B., \& Strauss, A. (1967). Thediscovery of grounded theory. Strategies for Qualitative Research. Mill Valley: The Sociology Press.

Grégoire, D. A., Barr, P. S., \& Shepherd, D. A. (2010). Cognitive processes of opportunity recognition: The role of structural alignment. Organization Science, 21(2), 413-431.

Gioia, D. A., \& Poole, P. P. (1984). Scripts in organizational behavior. Academy of management review, 9(3), 449-459.

Harris, S. G. (1994). Organizational culture and individual sensemaking: A schemabased perspective. Organization Science, 5(3), 309-321.

Hill, R. C., \& Levenhagen, M. (1995). Metaphors and mental models: Sensemaking and sensegiving in innovative and entrepreneurial activities. Journal of Management, 21(6), 1057-1074.

Hite, J. M. (2005). Evolutionary processes and paths of relationally embedded network ties in emerging entrepreneurial firms. Entrepreneurship theory and practice, 29(1), 113144.

Hult, G. T. M., \& Ketchen, D. J. (2001). Does market orientation matter?: A test of the relationship between positional advantage and performance. Strategic management journal, 22(9), 899-906.

Hult, G. T. M., Ketchen, D. J., \& Slater, S. F. (2005). Market orientation and performance: an integration of disparate approaches. Strategic Management Journal, 26(12), 11731181. 
Kendall, J. (1999). Axial coding and the grounded theory controversy. Western journal of nursing research, 21(6), 743-757.

Kirca, A. H., Jayachandran, S., \& Bearden, W. O. (2005). Market orientation: A metaanalytic review and assessment of its antecedents and impact on performance. Journal of marketing, 69(2), 24-41.

Kohli, A. K., \& Jaworski, B. J. (1990). Market orientation: the construct, research propositions, and managerial implications. The Journal of Marketing, 1-18.

Kraaijenbrink, J., Spender, J. C., \& Groen, A. J. (2010). The resource-based view: a review and assessment of its critiques. Journal of management, 36(1), 349-372.

Krueger Jr, N. F. (2003). The cognitive psychology of entrepreneurship. In Handbook of entrepreneurship research (pp. 105-140). Springer US.

Krueger Jr, N. F. (2007). The cognitive infrastructure of opportunity emergence. In Entrepreneurship (pp. 185-206). Springer Berlin Heidelberg.

Liao, J., Welsch, H., \& Tan, W. L. (2005). Venture gestation paths of nascent entrepreneurs: Exploring the temporal patterns. The Journal of High Technology Management Research, 16(1), 1-22.

Lounsbury, M., \& Glynn, M. A. (2001). Cultural entrepreneurship: Stories, legitimacy, and the acquisition of resources. Strategic management journal, 22(6-7), 545-564.

Lumpkin, G. T., Hills, G. E., \& Shrader, R. C. (2004). Opportunity recognition. Entrepreneurship: The way ahead, 73-90.

Martin, J. H., Martin, B. A., \& Minnillo, P. R. (2009). Implementing a market orientation in small manufacturing firms: from cognitive model to action. Journal of Small Business Management, 47(1), 92-115.

Mathieu, J. E., Heffner, T. S., Goodwin, G. F., Salas, E., \& Cannon-Bowers, J. A. (2000). The influence of shared mental models on team process and performance. Journal of applied psychology, 85(2), 273.

Mitchell, R. K., Busenitz, L., Lant, T., McDougall, P. P., Morse, E. A., \& Smith, J. B. (2002). Toward a theory of entrepreneurial cognition: Rethinking the people side of entrepreneurship research. Entrepreneurship theory and practice, 27(2), 93-104. 
Mitchell, R. K., Busenitz, L. W., Bird, B., Marie Gaglio, C., McMullen, J. S., Morse, E. A., \& Smith, J. B. (2007). The central question in entrepreneurial cognition research 2007. Entrepreneurship theory and practice, 31(1), 1-27.

Mitchell, Ronald K., Brandon Randolph-Seng, and J. Robert Mitchell. "Socially situated cognition: Imagining new opportunities for entrepreneurship research." Academy of Management Review 36.4 (2011): 774-776.

Morgan, N. A., Vorhies, D. W., \& Mason, C. H. (2009). Market orientation, marketing capabilities, and firm performance. Strategic Management Journal, 30(8), 909-920.

Narver, J. C., \& Slater, S. F. (1990). The effect of a market orientation on business profitability. The Journal of marketing, 20-35.

Narver, J. C., Slater, S. F., \& Tietje, B. (1998). Creating a market orientation. Journal of market-focused management, 2(3), 241-255.

Ozgen, E., \& Baron, R. A. (2007). Social sources of information in opportunity recognition: Effects of mentors, industry networks, and professional forums. Journal of business venturing, 22(2), 174-192.

Paulhus, D. L. (2002). Socially desirable responding: The evolution of a construct. The role of constructs in psychological and educational measurement, 49-69.

Pryor, C., Webb, J. W., Ireland, R. D., \& Ketchen Jr, D. J. (2015). Toward An Integration of the Behavioral and Cognitive Influences on the Entrepreneurship Process. Strategic Entrepreneurship Journal.

Raju, P. S., Lonial, S. C., \& Crum, M. D. (2011). Market orientation in the context of SMEs: A conceptual framework. Journal of Business Research, 64(12), 1320-1326.

Read, S., \& Sarasvathy, S. D. (2005). Knowing what to do and doing what you know: Effectuation as a form of entrepreneurial expertise. The Journal of Private Equity, 9(1), 45.)

Reijonen, H., Laukkanen, T., Komppula, R., \& Tuominen, S. (2012). Are Growing SMEs More Market-Oriented and Brand-Oriented?. Journal of Small Business Management, 50(4), 699-716. 
Rerup, C., \& Feldman, M. S. (2011). Routines as a source of change in organizational schemata: The role of trial-and-error learning. Academy of Management Journal, 54(3), 577-610.

Rosenberg, M. J. (1965). When dissonance fails: On eliminating evaluation apprehension from attitude measurement. Journal of personality and social psychology, 1(1), 28.

Rynes, S., \& Gephart Jr, R. P. (2004). From the Editors: Qualitative Research and the" Academy of Management Journal". The Academy of Management Journal, 454-462.

Sarin, S., \& Mohr, J. J. (2008). An introduction to the special issue on marketing of hightechnology products, services and innovations. Industrial Marketing Management, 37(6).

Schindehutte, M., Morris, M. H., \& Kocak, A. (2008). Understanding market-driving behavior: the role of entrepreneurship. Journal of Small Business Management, 46(1), 4-26.

Schutz, A., \& Luckmann, T. (1973). The structures of the life-world (Vol. 1). Northwestern University Press.

Shane, S. (2012). Reflections on the 2010 AMR decade award: delivering on the promise of entrepreneurship as a field of research. Academy of Management Review, 37(1), 1020.

Shapiro, C., \& Varian, H. R. (2013). Information rules: a strategic guide to the network economy. Harvard Business Press.

Shaver, K. G., \& Scott, L. R. (1991). Person, process, choice: The psychology of new venture creation. Entrepreneurship theory and practice, 16(2), 23-45.

Silverman, D. (2001). Interpreting qualitative data: Methods for analysing talk, text and interaction. London: Sage.

Sims, H. P., \& Gioia, D. A. (1986). The thinking organization. Jossey-Bass Inc Pub.

Sinkula, J. M. (1994). Market information processing and organizational learning. the Journal of Marketing, 35-45.

Slater, S. F., \& Narver, J. C. (1995). Market orientation and the learning organization. The Journal of marketing, 63-74. 
Stigliani, I., \& Ravasi, D. (2012). Organizing thoughts and connecting brains: Material practices and the transition from individual to group-level prospective sensemaking. Academy of Management Journal, 55(5), 1232-1259.

Strauss, A., \& Corbin, J. (1994). Grounded theory methodology. Handbook of qualitative research, 17, 273-285.

Strike, V., \& Rerup, C. (2016). Mediated sensemaking. Academy of Management Journal, 59(3), 880-905.

Sullivan, D. M., \& Marvel, M. R. (2011). Knowledge acquisition, network reliance, and early-stage technology venture outcomes. Journal of Management Studies, 48(6), 11691193.

Roersen, M. J., Kraaijenbrink, J., \& Groen, A. J. (2013). Marketing Ignorance and the Validity of Narver and Slater's MKTOR Scale in High-Tech Russian Firms. Journal of product innovation management, 30(3), 545-559.

Vaghely, I. P., \& Julien, P. A. (2010). Are opportunities recognized or constructed?: An information perspective on entrepreneurial opportunity identification. Journal of Business Venturing, 25(1), 73-86.

Van Raaij, E. M., \& Stoelhorst, J. W. (2008). The implementation of a market orientation: A review and integration of the contributions to date. European Journal of Marketing, 42(11/12), 1265-1293.

Venkataraman, S., Sarasvathy, S. D., Dew, N., \& Forster, W. R. (2012). Reflections on the 2010 AMR decade award: Whither the promise? Moving forward with entrepreneurship as a science of the artificial. Academy of Management Review, 37(1), 21-33.

Walsh, D. (1998). Doing ethnography. Researching society and culture, 217-232.

Webb, J. W., Ireland, R. D., Hitt, M. A., Kistruck, G. M., \& Tihanyi, L. (2011). Where is the opportunity without the customer? An integration of marketing activities, the entrepreneurship process, and institutional theory. Journal of the Academy of Marketing Science, 39(4), 537-554.

Weick, K. E. (1995). Sensemaking in organizations (Vol. 3). Sage. 
Weick, K. E., Sutcliffe, K. M., \& Obstfeld, D. (2005). Organizing and the process of sensemaking. Organization science, 16(4), 409-421.

Weick, K. E. (2012). Organized sensemaking: A commentary on processes of interpretive work. Human Relations, 65(1), 141-153.

West, G. P., \& Noel, T. W. (2009). The impact of knowledge resources on new venture performance. Journal of Small Business Management, 47(1), 1-22.

Whalen, P. S., \& Akaka, M. A. (2016). A dynamic market conceptualization for entrepreneurial marketing: the co-creation of opportunities. Journal of Strategic Marketing, 24(1), 61-75.

Wise, S. (2013). The impact of financial literacy on new venture survival. International Journal of Business and Management, 8(23), 30.

Wry, T., Lounsbury, M., \& Glynn, M. A. (2011). Legitimating nascent collective identities: Coordinating cultural entrepreneurship. Organization Science, 22(2), 449-463.

Zott, C., \& Huy, Q. N. (2007). How entrepreneurs use symbolic management to acquire resources. Administrative Science Quarterly, 52(1), 70-105. 


\section{ChAPTER 5}

MAKING SENSE OF THE BUSINESS MODEL: A COGNITIVE INVESTIGATION INTO

THE USE OF THE BUSINESS MODEL ARTIFACT BY ENTREPRENEURS

Gabi A. Kaffka, University of Twente

Jeroen Kraaijenbrink, University of Twente

Aard J. Groen, University of Twente

History of the manuscript: This chapter is a modified version of a peer-reviewed paper that was presented at the HTSF conference in May 2012 in Amsterdam, The Netherlands. 


\section{ABSTRACT}

This study focuses on entrepreneurial sensemaking of the business model artifact. We distinguish between four general purposes for which the business model artifact can be used. These are respectively understanding, communicating, analyzing and developing the venture's value creation logic. To examine how entrepreneurs make sense of the business model artifact in terms of those different purposes, we carried out a qualitative analysis of interviews with 85 entrepreneurs who are involved in business opportunity development. In particular we looked at the effect of human capital in the form of educational background, and distinguish between STEM disciplines (science, technology, engineering and mathematics) and non-STEM disciplines. The results show that entrepreneurs use the business model artifact mainly for the purposes of understanding and developing their value creation logic, and that STEM entrepreneurs use the business model artifact significantly more for communication and analytical purposes than non-STEM entrepreneurs. We contribute to the literature by demonstraing empirically how entrepreneurs make sense of the business model artifact differently, and how human capital affects entrepreneurial sensemaking during opportunity development.

\subsection{INTRODUCTION}

A business model is a simplified representation of the venture's value creation logic. It characteristically consists of a value proposition as well as the customer segment which is targeted, how the value is created and with what financial consequences (Osterwalder, 2004, Osterwalder \& Pigneur, 2005; Chesbrough, 2010). A business model is seen as the organizational and financial 'architecture' of a business (Teece, 2010) and used for the depiction of value creation processes in various industries, sectors, and specific firm products or services (Magretta 2002; Al-Debei and Avison 2010; Teece 2010). Because a firm's business model is critical to enterprise success in terms of profitable and lasting value creation (Teece, 2010), there is a lot of attention for the design and change of a venture's business model from academic and practitioners (Zott et al., 2011). It has also become a central topic in entrepreneurship studies (Bock, 2011; Trimi et al., 2012). 
The business model is an artifact that can be used for different purposes. Research shows that the way entrepreneurs use their business model affects the way their business opportunity develops (Martins et al., 2015). In order to examine this purposeful use, we look at sensemaking processes regarding the use of the business model artifact by entrepreneurs during opportunity development.

In particular, we are interested in the role that educational background of entrepreneurs plays in sensemaking of different purposes for which the business model artifact can be used. Studies have shown that the way in which entrepreneurs identify and prepare a business opportunity is affected by the entrepreneur's human capital (Davidsson \& Honig, 2003; Brinckmann \& Kim, 2015). Entrepreneurs with a background in the academic disciplines of science, technology, engineering and mathematics (the so-called STEM disciplines) tend to focus on the technical aspects of their business idea (Berry, 1996). The 'technical mindset' of STEM entrepreneurs can impede their focus on value for the customer, which in turn causes a 'management' or survival challenge for the novel business opportunity (West \& Noel, 2009; Schindehutte, Moris \& Kocak, 2008). This indicates that entrepreneurs with an educational background in the STEM disciplines (henceforth: STEM entrepreneurs) make sense of their business opportunity differently than entrepreneurs from other educational disciplines. But as yet, a systematic comparison of sensemaking processes of entrepreneurs with different educational backgrounds is as yet lacking.

Our central research question is the following: How do entrepreneurs make sense of their use of the business model artifact during opportunity development?

To answer this research question, we distinguish between four purposes for which the business model can be used and which are respectively: understanding, communicating, analyzing and developing the venture's value creation logic. We draw on qualitative data from open-ended interviews with 85 entrepreneurs who are involved in business opportunity identification and preparation. Using the sensemaking perspective we examine the entrepreneurs' reports regarding their use of the business model artifact. The results of our analysis show that entrepreneurs make sense of the business model artifact mainly for the purposes of understanding and developing their value creation logic. In addition, we saw that STEM entrepreneurs report significantly more sensemaking for the purposes of communication and analysis than non-STEM entrepreneurs. 
With this study we aim to contribute to the literature in the following ways. Firstly, we demonstrate empirically for which purposes the business model artifact is used by entrepreneurs during opportunity development. Secondly, this research yields deeper insights into the way that education affects sensemaking processes, specifically regarding the use of the business model artifact during opportunity development. By doing so, we contribute to theory-building on the way in which human capital affects entrepreneurial cognition.

This chapter is set up as follows. First, we elaborate on the theoretical approach which is employed for the analysis of sensemaking of the business model artifact. Next, we present the method used in this study to answer our research question. Then we present the results of our analysis, before turning to the conclusion and discussion of those results. Lastly, we discuss the limitations of this study and advance recommendations for future research.

\subsection{THEORY}

\section{THE BUSINESS MODEL ARTIFACT IN THE LITERATURE}

Despite an upsurge in studies on business models and numerous definitions of business models, there are still hardly any theoretical groundings of the business model in economics or business studies (Teece, 2010). As Arend (2013) underlines, the idea behind a 'model' of value creation is of great importance, especially in the case of nonmonetary exchanges. However the author also criticizes that the term 'business model' (as a description of how a traditional venture operates) lacks theoretical ground. Similarly, Zott et al. (2011) suggest scholars have yet to develop a common and widely accepted language on the business model artifact which allows research to effectively draw on the works of others. While definitions of the business model vary in scope and conceptual focus, the majority of them focus on value creation and value offerings of an individual enterprise (Lambert \& Davidson, 2013).

A business model articulates the logic and provides data and other evidence that demonstrates how a business creates and delivers value to customers (Teece, 2010). A business model is positioned between the competitive strategy and business processes of organizations (Magretta, 2002). It represents the 'organizational and financial architecture' of a company (Teece, 2010). While it is more generic than a business 
strategy (Teece, 2010), it helps to make a selection where and how in the value chain value is created (Chesbrough, 2010). The term business model is used in various industries and sectors (Magretta 2002; Osterwalder et al., 2005; Al-Debei \& Avison 2010). A venture's business model satisfies both the need for planning and the call for flexibility in the modern networked market (Osterwalder \& Pigneur, 2005), recently also recognized for its ability to bring 'the customer back into the spotlight for both strategy and entrepreneurship scholars' (Demil et al., 2015).

The notion of the business model was spawned by a rapid increase in use, and dropping costs of, information technology (IT) in (post)modern businesses. This technology enabled the rise of new markets, with products and services in multimedia, online entertainment and other fields (Teece, 2010). These new markets created the possibility to define a variety of new business models, in particular in the realm of ecommerce and e-business (Osterwalder, 2004; Osterwalder \& Pigneur 2005; Teece, 2010). Examples are the innovative business models of Facebook (digital platform) or Google (online search engine) who profit from providing (paid) access to user information to third parties.

In empirical research the business model artifact has been analyzed in three ways: the business model as the basis for enterprise classification, the business model and its relationship with enterprise performance, and business model innovation (Lambert \& Davidson, 2013). Osiyevskyy \& Dewald (2015) found that business models innovation or change is typified by either explorative adoption of a disruptive business model or the exploitative strengthening of the existing business. Also, business model change is affected by third parties, such as venture capital firms or outside CEOs (Gerasmymenko et al., 2015).

There also is business model as a conceptual artifact, as meta-model which depicts the general 'building blocks' that a venture's business model consists of (Osterwalder et al., 2005). Osterwalder et al. (2005) describe three categories into which the literature on business models can be classified and that can be hierarchically linked to one another. On the most abstract (and hierarchically highest) level, there is literature in which the business model artifact is described as an abstract overarching concept which depicts all real world businesses. The second category contains literature in which a number of different abstract types of business models are described, each with a set of common characteristics, for example the type of online ventures such as Amazon or Dell. The third category on the bottom of the hierarchy consists of literature in which 
authors present aspects of or a conceptualization of a particular, real world business model.

On the highest abstraction level of Osterwalder et al.'s (2005) hierarchy the business model artifact is not a concrete representation of a real-existing firm's value creation logic, but must be seen it its capacity of being a conceptual model. On this level, the business model artifact represents an artifact of which entrepreneurs can make sense in different ways.

\section{SENSEMAKING OF THE BUSINESS MODEL ARTIFACT}

Entrepreneurial opportunity development involves previously unknown and unknowable value creation processes and is therefore characterized by uncertainty (Knight, 1921; Sarasvathy, 2001; McMullen \& Shepherd, 2006). In uncertain situations, actors use sensemaking because it facilitates the ordering of the chaotic flux of reality events (Weick et al., 2005). Sensemaking is recognized to be essential to opportunity development (Mitchell et al., 2011). It is concerned with interpretative actions during novel situations, also business opportunity identification and preparation (Hill \& Levenhagen, 1995). By means of sensemaking, actors create order in the incessant chaotic "flux" of information that they are confronted with in day-to-day life.

Sensemaking is "the primary site where meanings materialize to inform and constrain" (Weick et al., 2005). Meaning is an operation of intentionality which becomes visible to the reflective glance (Schwandt, 2005) and driven by the individual actor's seeking of plausibility of events and situations (Weick, 1995; Humphreys et al., 2012). Those meanings are developed during a process which consists of picking up cues ('signaling', interpreting those cues as meaningful ('bracketing'). Signaling and bracketing leads to the categorization ('labeling') of events or situations (which create, inform and constrain an individual's identity and action (Weick, 1995; Weick et al., 2005; Maitlis \& Sonenshein, 2010). Actors signal, bracket and label in order to fit information which is relevant to them into a plausible reality, a legitimate identity or a purposeful activity (Weick, 1995).

Sensemaking processes can involve the use of (im)material artifacts (Garud \& Guiliani, 2013). The business model as a conceptual model is such an artifact. The role of artifacts in sensemaking has been studied by Stigliani \& Ravasi (2012) who examined the role of artifacts in the articulation of meaning during value creation processes in organization. The authors propose that individuals use various types of artifacts to 
support the construction of new understandings while engaging in sensemaking and sensegiving processes, for example by using PowerPoint presentations, drawings, models, and prototypes which facilitate the exchange of understandings across involved stakeholders.

Artifacts can be discursive - such as concepts and models - or material, such as visual or textual artifacts. Artifacts have received increasing attention from entrepreneurship scholars (Venkataraman et al., 2013). The business model artifact for example facilitates an entrepreneur's enactment of connecting "an underlying business idea with the future organization of concrete resources" (Selden \& Fletcher, 2015).

In this study, we analyze entrepreneurial sensemaking of the business model artifact, in terms of its purposeful use during opportunity development. The different purposes for which the business model artifact can be used are presented below.

\section{PuRPoses OF THE BUSINESS MOdEL ARTIFACT}

The literature on business models shows that the business model artifact is used for different purposes. Traditionally, a venture's business model had the purpose of the design of the value creation logic (Magretta 2002; Teece, 2010) but there is a considerable amount of research which examines how the business model artifact is used for innovating or changing the business opportunity (Lambert \& Davidson, 2013). For example, Guptha \& McMillan (2004) study the way entrepreneurial leaders enact the transformation of the venture's business model, while Spieth et al. (2014) distinguish between three different roles of a venture's business model, which include that of explaining the business, running the business and developing the business.

In order to obtain an overview of the different purposes identified in the literature regarding the purposes for which the business model artifact we carried out a literature review to obtain an overview of the. The particular focus was on different uses of the business model artifact by entrepreneurs in the start-up phase. As a starting point, we used two special issues of high-ranking academic journals as initial literature sources, one from the Strategic Entrepreneurship Journal (SEJ) and the other one from the journal Long Range Planning (LRP). Both are leading journals in the field of strategic management, with impact factors of 6.36 (SEJ) respectively 2.936 (LRP) in 2015 . The reason for choosing these two literature sources is that they are both authoritative sources of scientific studies, and both contain articles with relevant references to other articles on the topic of business models. 
Drawing on the articles comprised in those two special issues we employed the snowball method to trace relevant articles cited in these two initial literature sources. To do so we carried out a search within the studies listed in the bibliographies of the articles contained in the special issues. We focused on the identification of those studies which examine the (instrumental) roles, functions or purposes for which entrepreneurs use a business model. Next, we carried out a bibliography analysis with the same aim in the bibliographies of those articles. In that sense, the two initial literature sources provided us with a peer review of articles relevant for our study. By doing so, we sought to increase the reach of our search to relevant studies.

We identified a number of purposes for which the business model artifact is used by entrepreneurs and which have been examined in the entrepreneurship literature. The publications listed in table 5-1 contain exemplary studies that convey how the business model artifact is used (column 3), as well as the more general purposes those uses reflect (column 4). 


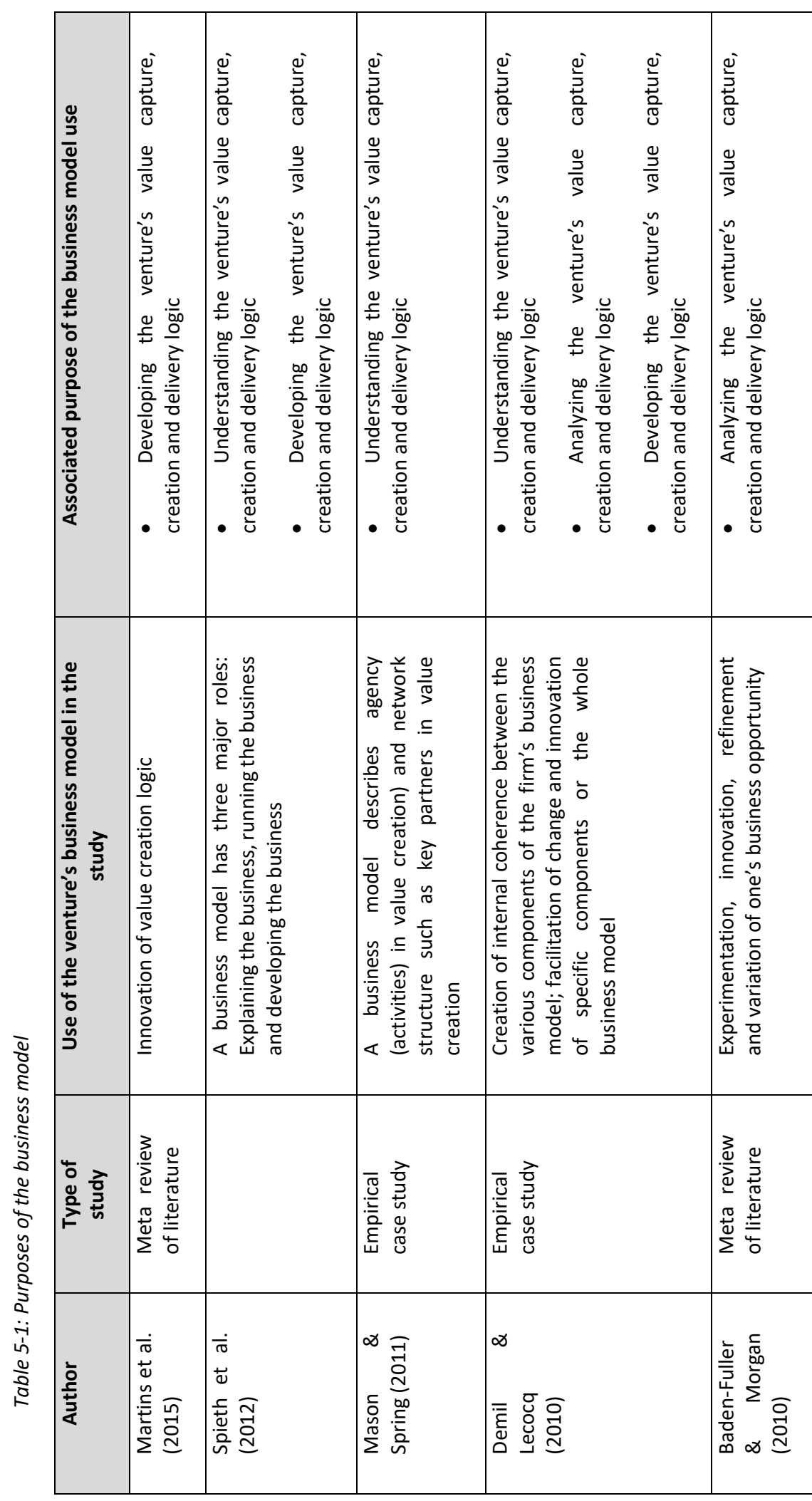




\begin{tabular}{|c|c|c|c|c|c|}
\hline 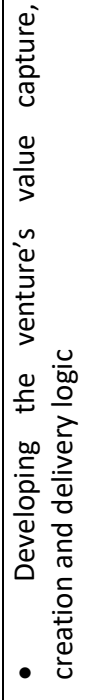 & 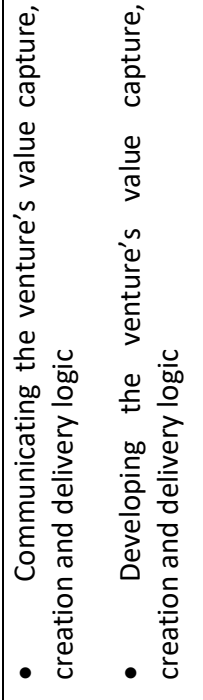 & 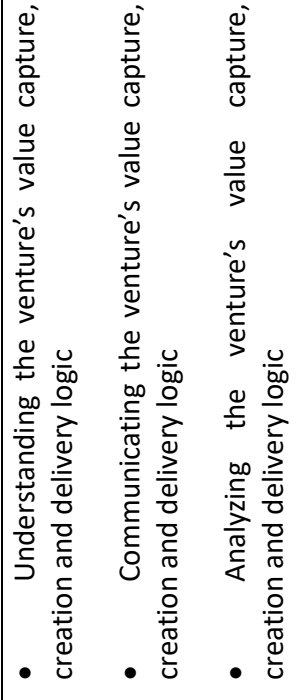 & 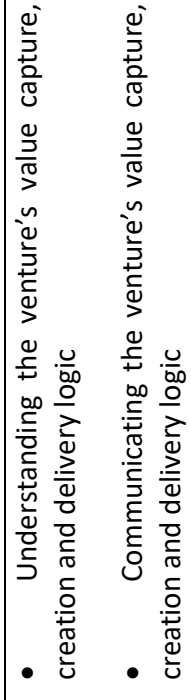 & 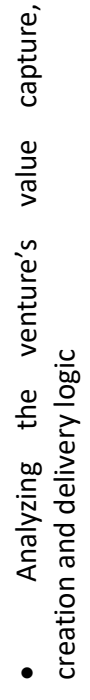 & 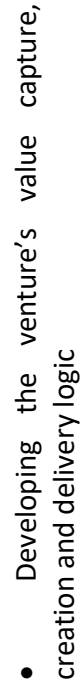 \\
\hline & 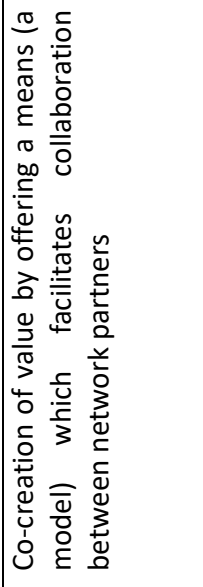 & 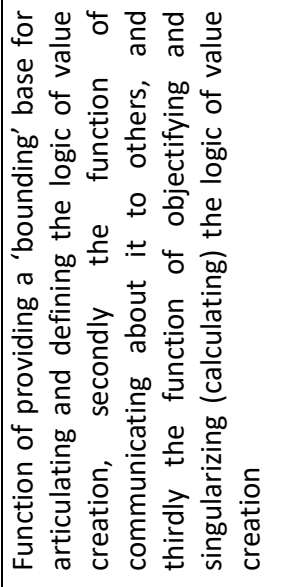 & 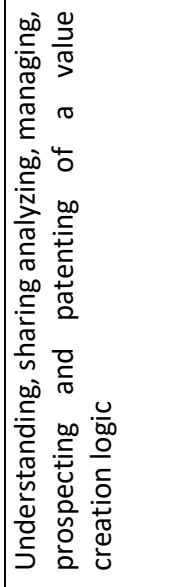 & & \\
\hline & 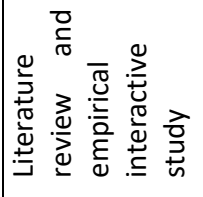 & 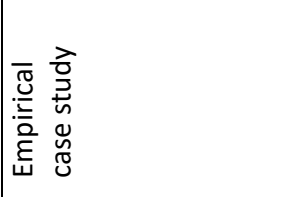 & 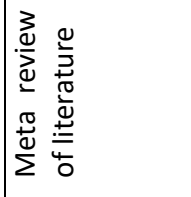 & & \\
\hline & 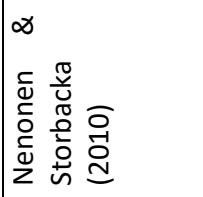 & 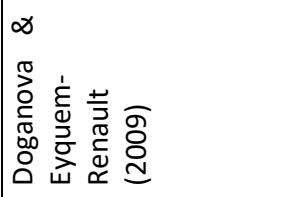 & 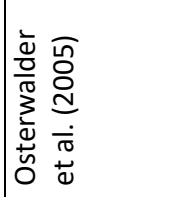 & & \\
\hline
\end{tabular}


One purpose for which the business model artifact is used is that of understanding the venture's value creation logic. When the business model artifact is used in this way, it functions as bounding tool of the business opportunity, by facilitating one's 'capturing' of a business model. An example is a case-study by Doganova \& Eyquem-Renault (2009) who find that the business model artifact provides a 'bounding' base for articulating and defines the logic of value creation.

The purpose of 'understanding value' can for example be achieved by visualizing, defining and designing the venture's business model or the different elements of it (Osterwalder \& Pigneur, 2005). It also includes the identification of relevant stakeholders in the definition of the value chain and the customer segment, and subsequently affects the articulation of activities as a result of defining the venture's value creation logic. This can even lead to the articulation of actions as a result of the business model design; for example, Mason \& Spring (2011) describe the role of the business models in shaping action.

The second purpose for which the business model artifact is used according to the literature is the 'communicating purpose'; for example when the business model artifact is used for narrative or sharing tool during the presentation of the venture's business model to third parties. As Doganova \& Eyquem-Renault (2009) show, a venture's business model can be used to communicate about the venture's specific value creation logic to others. For example, entrepreneurs use their venture's business model to explain its value creation logic to investors, supplier or employees. Similarly, Osterwalder \& Pigneur (2005) describe the way the venture's business model can be used to share the venture's value creation logic with others. And Spieth et al. (2014) differentiate three major roles that a venture's business model plays, among which the role of explaining how an existing or future venture is to generate profit to external shareholders of that venture, such as investors, media, customers or partners, as well as internal employees.

The 'communicating purpose' of the business model artifact facilitates the presentation of the venture's value creation logic to third parties and thus obtain valuable feedback. This also contributes to value co-creation. For example, Nenonen \& Storbacka's (2010) research investigates firms' business models as a broader conceptualization of value co-creation. The authors draw on the notion of co-creation of 'discursively legitimated market spaces' in order to create value, and underline the role of negotiation among market actors through business models. In doing so, the authors point towards the communication purpose which the business model artifact fulfills. 
A third general purpose we identified in the literature on the uses of the business model artifact is the 'analyzing purpose'. This purpose is associated with activities such as measuring, observing or comparing a venture's business model - the latter in relation to other (possible) business models. For example, Demil \& Lecocq (2010) describe the business model artifact as a blueprint for analyzing the coherence between core business model components. And Baden-Fuller \& Morgan (2010) distinguish the function of experimentation of a business model; since this activity involves an examination of the consequences of that value creation logic (potentially also in comparison to other possible value creation logics) it reflects the analytical purpose for which the venture's (possible) business model is used in this scenario.

Actors also draw on the 'analyzing purpose' of the business model artifact when they use their venture's business model to compare the current customer segment to potential future segments, or to measure the success of previous value creation processes. Used for the 'analyzing purpose', a venture's business model also facilitates the identification of measures to improve management, the tracing of issues of the firm's value creation logic over time or the comparison of one's business model with that of competitors. Doganova \& Eyquem-Renault (2009) find that a venture's business model can be used to objectify and singularize the logic of value creation, such as making it calculable for accountancy purposes.

The fourth general purpose for which a business model artifact can be used is the 'developing purpose'. For example, an entrepreneur can use the business model artifact to design, plan or change the venture's value creation logic, but also for reacting to (changing) markets or aligning one's value creation logic with a market. Baden-Fuller \& Morgan (2010) differentiate not only the 'experimentation function' mentioned above, but also a number of functions which relate to the 'developing purpose' of the business model artifact; these contain innovation, refinement and variation of the venture's business model. Similarly, Demil \& Lecocq (2010) describe the transformational function of the business model artifact in addressing change and innovation. And Spieth et al. (2014) distinguish the role of developing the business model as central to a venture's business model. In this role, the business model function is to support management in defining and developing the firm's strategy.

In its 'developing' purpose the business model artifact is used to foster innovation, for example in order to react to a changing competitive landscape. In this function, the entrepreneur can also use the business model to align it with the firm's strategy and 
technology or to simulate and test various business models in order to create a business model portfolio, so that different business models are at hand in the face of changing environmental circumstances or when prospect new business activities. For example, Osterwalder \& Pigneur (2005) identify the functions of managing, prospecting and patenting of the firm's value creation logic.

The four different purposes which are described above and for which the business model artifact is used by entrepreneurs have all been emerged in empirical studies on the use of the business model artifact. We contend that those four purposes are sufficiently broad to cover a wide range of usage of the business model artifact which also distinctive from one other. In this study, we examine entrepreneurial sensemaking of the business model artifact by looking at the way entrepreneurs report their use of the business model artifact for these four different purposes. Summaring, these general purposes are:

- Understanding the venture's value creation logic

- Communicating the venture's value creation logic

- Analyzing the venture's value creation and delivery logic

- Developing the venture's value creation logic

\section{THE ROLE OF EDUCATION IN OPPORTUNITY DEVELOPMENT}

In this study we are particularly interested in the role which educational background plays in how entrepreneurs make sense of the different purposes for which the business model artifact can be used.

Entrepreneurs make sense of events and situations based on their cognitive ability to 'connect the dots', to bring together bits of previously unconnected information, in order to create novel value (Baron \& Ensley, 2006). This ability is affected by the entrepreneur's prior knowledge (Grégoire et al., 2010), such as education, work and entrepreneurial experience (Smith, 1967; Davidsson \& Honig, 2003; Haynie et al., 2009). Given the strong influence of one's prior knowledge structures on the perception and interpretation of information by entrepreneurs (Baron \& Ensley, 2006; Grégoire et al., 2010), it is likely that the formal educational background of an entrepreneur also influences his or her sensemaking of business model artifact. Therefore we are particularly interested in the effect of formal education on business model use by entrepreneurs. 
Studies have shown that entrepreneurs with a background in the so-called STEM disciplines (science, technology, engineering and mathematics) tend to focus on the technical aspects of their business idea. For example, Berry (1996) described how firms which are dominated by technologists in the management are likely to be technologydriven instead of market-driven. This is due to those managers' 'technical' educational background which can form a threat to survival and growth of small high-tech firms. And Knockhaerdt et al. (2010) write: "A common concern with entrepreneurs [...] is that they often lack commercial experience, resulting in a tendency to focus only on the technical aspects of innovation." (p. 790).

Other studies have shown that entrepreneurs select team members which have the same (technical) educational background, which results in lacking commercial experience in the venture (Ensley \& Hmieleski, 2005; Franklin, Wright, \& Lockett, 2001). The ensuing predominantly 'technical mindset' can impede the entrepreneur's focus on value for the customer, which in turn causes a 'management' or survival challenge of the novel business opportunity (West \& Noel, 2009; Schindehutte, Moris \& Kocak, 2008). These findings point to the influence of prior education of the management team of a venture on the commercial success of that venture.

\subsection{METHOD}

\section{RESEARCH DESIGN}

To analyze entrepreneurial sensemaking about the purposeful use of the business model artifact, we carried out a qualitative analysis of interview data collected from entrepreneurs who are involved in business opportunity development.

\section{SetTING}

This study took place within a business incubation/acceleration program called the Venture Lab Twente (VLT). It is a one-year program which provides institutional support for novice and experienced entrepreneurs in the Netherlands who are in the process of developing a novel business opportunity, offering resources in the form of office facilities, trainings and coaching and panel feedback.

The program contains various instances in which the business model is trained. There is a basic training on business modeling which lasts one afternoon and is offered 
at least twice during the one-year program. Entrepreneurs who participate in the training are informed about various aspects of a business model and are encouraged to formulate and use a business model for their own business idea and for different purposes. In this way, entrepreneurs are not only informed about the possibilities for business model use, but also stimulated to immediately design their venture's value creation logic in practice, and thus get acquainted with the business model artifact.

By drawing respondents from a sample which has knowledge of the business model artifact, the awareness of this artifact by entrepreneurs in our sample is ensured while avoiding to affect - and bias - their reported sensemaking about their use of the business model artifact.

\section{Measuring education}

In this study, we analyze the use of the business model for different purposes by entrepreneurs. In addition to the qualitative data in the form of interviews about the utilization of the business, we draw on quantitative data about the respondents: their age, gender and education. Age and gender are coded as a nominal variable respectively as a binary variable.

Education is coded in terms of two dimensions: the level of education and the type of education of respondents. Level of education is coded nominally, from primary school to post-graduate education. The other educational variable - type of education - is coded binary, in terms of various educational field which lead to a professional expertise.

For a comprehensive coding of professional fields we drew on the International Standard Classification used by the United Nations Educational, Scientific and Cultural Organization (UNESCO). We categorized educational fields into technical education on the one hand, and non-technical education on the other hand. Drawing on Berry's (1996) descriptions of technical disciplines, the distinction is based on the degree of affinity of the professional field with natural sciences and engineering, the classical so-called 'technical' studies. This distinction refers to the difference in studies focusing on the physical world and those which focus on the study of human behavior or the products thereof, loosely based on the commonly used Anglo-Saxon distinction between sciences respectively social sciences and humanities. In this study, we employ a dichotomous categorization whereby the so-called 'STEM' disciplines contain those with educational content relating to natural sciences, technology, engineering and mathematics; and 'nonSTEM' disciplines which encompass all other educational disciplines, such as social sciences, liberal arts and humanities. We present an overview of these two categories 
of educational disciplines in table 5-2. In our study, these two educational sub-samples are designated respectively STEM and non-STEM population of entrepreneurs.

Table 5-2: Categorization of education fields in STEM and non-STEM disciplines

\begin{tabular}{|c|c|}
\hline STEM disciplines & Non-STEM disciplines \\
\hline $\begin{array}{l}\text { - } \text { Life sciences } \\
\text { - } \text { Physical sciences } \\
\text { - } \text { Mathematics and statistics } \\
\text { - } \text { Engineering and engineering trades } \\
\text { - } \text { Manufacturing and processing } \\
\text { - } \text { Agriculture, forestry and fishery } \\
\text { - } \text { Veterinary } \\
\text { - } \quad \text { Health if biochemical/biomedical }\end{array}$ & 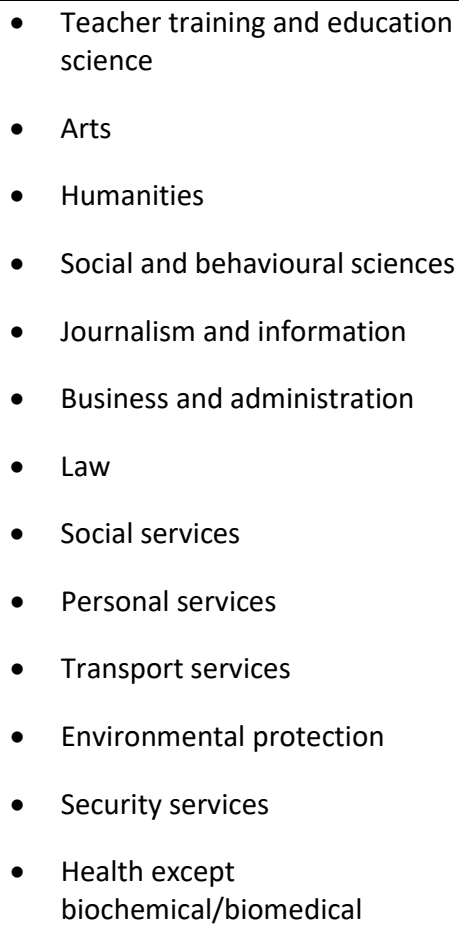 \\
\hline
\end{tabular}

\section{DATA COLLECTION}

The interviews were scheduled at the moment when entrepreneurs exited the program. We chose to carry out structured, open-ended interviews in order to catch the entrepreneur's reports about their meanings, impressions and perceptions of using the business model. Since all interview studies with a high data validity must satisfy the criterion of low-inference descriptors (Silverman, 2001) all interviews were audio-taped and transcribed.

The qualitative research approach chosen in this study provides insights into subjective experiences of an actor however it also raises the issue of biased data collection. Biases in self-reports of study subject are a relevant issue in entrepreneurship research. For example, Bird (2014) pointed towards desirable answering by 
entrepreneurs as a noticeable methodological challenge in cognitive studies in entrepreneurship.

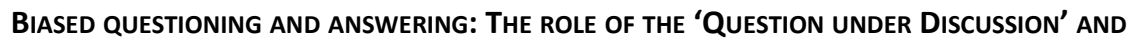
SOCIAL DESIRABILITY

Research shows that people have a tendency to give overly positive selfdescriptions, for example the desirable or most moderate response to a question, which relates to the phenomenon of socially desirable answering, SDR (Paulhus, 2002). Social desirability is defined as evaluation apprehension or the respondent's tendency to respond in a "typical" fashion to avoid appearing "different" (Rosenberg, 1965; Shaver \& Scott, 1991). Bird (2014) emphasizes that the bias of social desirable answering is particularly relevant when assessing entrepreneurs' thoughts, plans, decisions and actions. For example, research shows that entrepreneurs display optimism and dispositional positive affect which can lead to biased recall of information (Hmieleski \& Baron, 2009).

The institutional support context in which this study is embedded implies a sample bias. Entrepreneurs in a business incubation environment self-selectively volunteered for access to (intangible) resources provided in such a context. This context could lead to a higher social desirability bias in the entrepreneurs' reports, who seek to demonstrate proficiency and versatility in leveraging the offered (intangible) resources, in this case their use of the business model artifact they have been introduced to. In the case of this study, we recognize that an entrepreneur might be affected by what Bird (2014) describes as "his or her desire to be a good (or bad) experimental subject" (Bird, 2014: p.123). Interviews containing explicit questions about business model use could lead to biased answers regarding the purposes for which they use the venture's business model artifact, and reducing the validity of our study.

In order to control for this bias, we collected two samples of interviews. From our sample we drew two subsamples to compare the reported purposes and control for significant differences in reported purposes. Both subsamples are part of our final analysis and contain the open-ended, structured questions about personal and venture development of the entrepreneur. In one subsample, the interview questionnaire contains no explicit questions about the respondent's use of the business model. In the other subsample, we asked additional structured questions about business model use to the interview. Appendix 1 contains the interviews questions as well as the additional business model questions in the interviews. 
By comparing the way entrepreneurs report business model purposes when asked directly about it and when not, we can identify the potential effects of cognitive biases such as that of social desirability in responses.

\section{SAMPLE CHARACTERISTICS}

In total, the sample contains 85 respondents. They are from the population of entrepreneurs who participated in the VLT incubation/acceleration program during the years 2011 and 2012. During the two years of interviewing, ten to twenty entrepreneurs completed and exited the support program every three months. We interviewed only entrepreneurs who had fully completed and exited the VLT program. The interviews were collected within a month that they finished the program.

Since we are interested in how entrepreneurs make sense of the purposes of business model during the phase of opportunity identification and preparation, we excluded interviews with entrepreneurs who did not develop a novel business opportunity per se at the time of interviewing. 9 respondents from our sample are excluded for this reason. This concerns family entrepreneurs, 'intrapreneurs' (who work as employees in an existing firm) and entrepreneurs who had abandoned gestation activities altogether during the time of the incubation/acceleration program.

Our final sample contains 76 respondents, of which eight are female entrepreneurs. The average entrepreneur in this sample is 45 years old (with a standard deviation of 10 years), has 14 years of work experience before making the decision to become an entrepreneur (standard deviation: 10 years) and 7 years of previous entrepreneurial experience (standard deviation: 7 years). 4 entrepreneurs in our sample are (part-time) employed, all other respondents are engaged full-time in the development of their business opportunity. 19 respondents in this sample are serial or portfolio entrepreneur.

Overall, the average educational level of entrepreneurs in our sample is high. More than half of the sample has at least an academic education on the graduate level. 32 respondents in the sample have obtained a doctorate degree of which 19 respondents in field of natural science or technology. The Netherlands are known for a high educational level (OECD, 2014) which is reflected in this sample. The high level of education is also likely affected by the research setting; an incubation/acceleration program which is affiliated to a technical university and thus attracts its (post)graduate students who wish to pursue the commercialization of scientific research results. 


\section{DATA ANALYSIS}

We carried out a discourse analysis of the interviews. Discourse analysis is an interpretive process in which key categories, recurrent themes and terms help to organize the data (Tonkiss, 1998; Silverman, 2001). In this case, these key themes pertain to activities for which the business model is reported to be used, and associated purposes of business model used as identified in the theory section. In order to discern sensemaking processes we also draw on existing analytical concepts from the sensemaking literature, specifically the role of discursive devices.

Sensemaking processes are reflected in actors' justifications which bind people to actions that are consistent with them and which tend to recur, stabilize and serve as resource for dominant stories (Weick, 2012). Justifications or plausible accounts of certain events or phenomena by entrepreneurs - in this case their purposeful use of the business model artifact - are associated with the use of symbols and other discursive devices to establish identity and legitimacy of the business (Zott \& Huy, 2007; Clarke, 2011; Clarke \& Cornelissen, 2011). Discursive devices or linguistic tools are used in sensemaking by actors (Hill \& Levenhagen, 1995; Stigliani \& Ravasi, 2012). Discursive devices can be effectively used for making and conveying sense during entrepreneurial value capture, creation and delivery.

Cornelissen \& Clarke (2011) point to the importance of discursive devices for entrepreneurs during business opportunity development. Discursive devices pertain to metaphors, analogies, or even juxtapositions. In addition, entrepreneurs use images and other symbols in what has been described in 'symbolic impression management' during business opportunity development. (Zott \& Huy, 2007). The use of images involve sensemaking processes which instill those images with meaning (Stigliani \& Ravasi, 2012). For example, linguistic frames, labels and metaphors are used to convey the identity of an organization or company (Navis \& Glynn, 2010).

When presenting the results of our analysis and where it is applicable, we describe the type of discursive element, or the association with entrepreneurial identity or legitimacy identified in the entrepreneur' reports of their use of their venture's business model.

\section{CODING PROCESS}

We coded entrepreneurs' reports on their use of the business model in terms of activities related to the different purposes of the business model which are described in 
the theory section For our analysis, a codebook was designed which contains the operationalization of the purposes identified in the theory section and their empirical indicators in terms of reported behavior or motivation (see table 3). A codebook facilitates researcher triangulation and ensures inter-rater reliability. The codebook used during the analysis is shown in table 5-3.This codebook consists of key signal words associated with activities and meanings identified in the discourse which reflect the use of the business model for a particular purposes or motivations for using it in a certain way. In that way, the codebook ensured the validity, reliability and thus transparency of the analysis.

The unit of analysis is an individual sentence as well as a logical 'chain' of sentences in the transcripts of the interviews. A logical chain of sentences is defined as a number of sentences which semantically pose an integral (interrelated) unity. For example, this occurs when an account consists of several sentences which reference back to the same subject. 


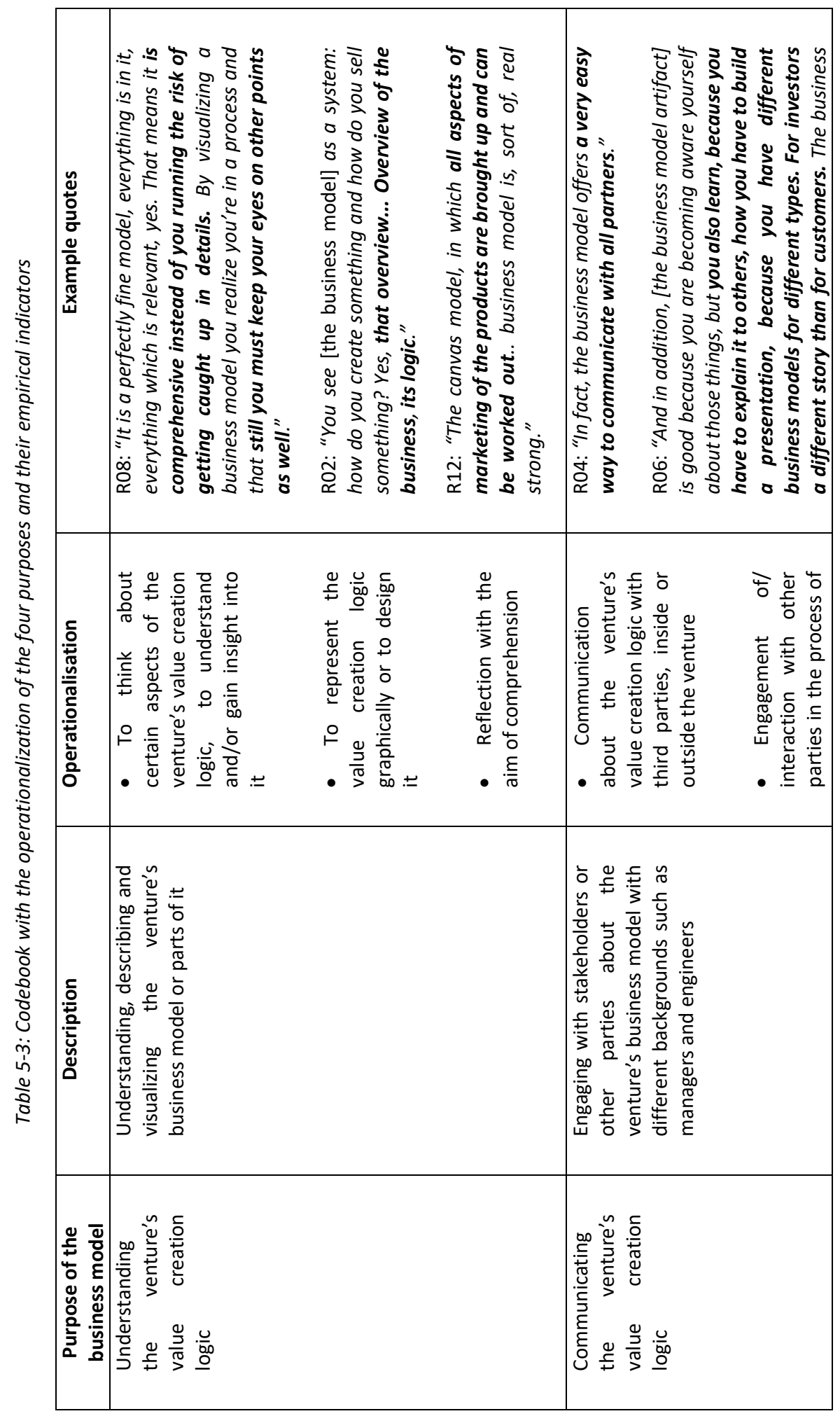




\begin{tabular}{|c|c|c|c|c|}
\hline 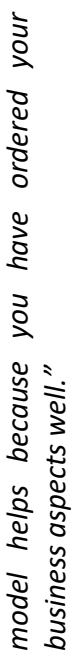 & 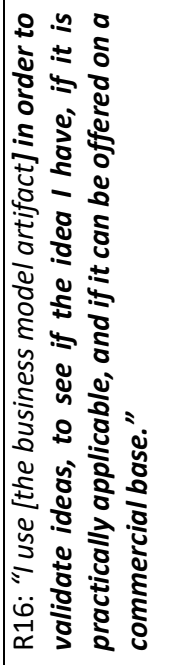 & 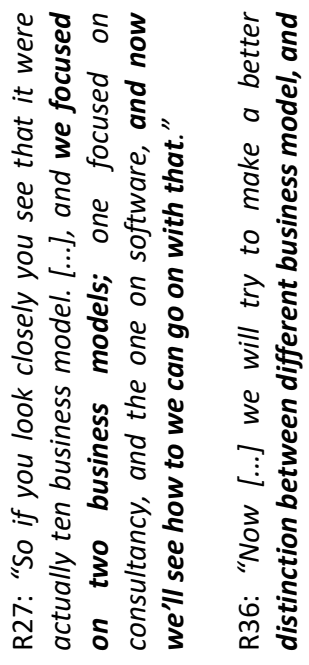 & & \\
\hline 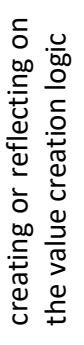 & 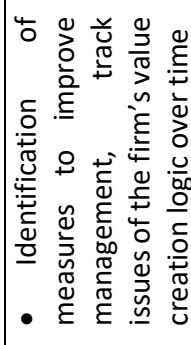 & 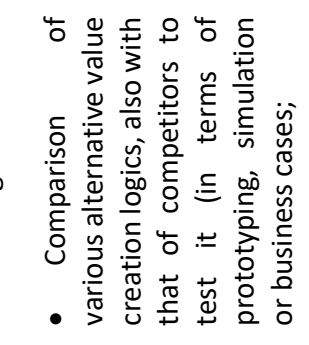 & 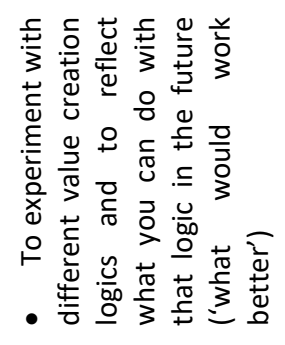 & 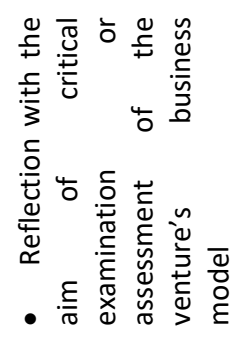 \\
\hline & 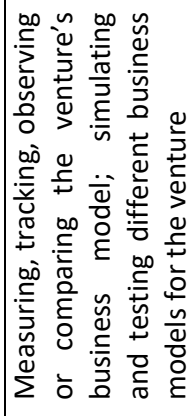 & & & \\
\hline & 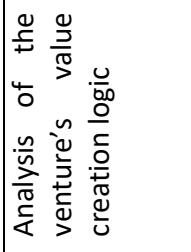 & & & \\
\hline
\end{tabular}




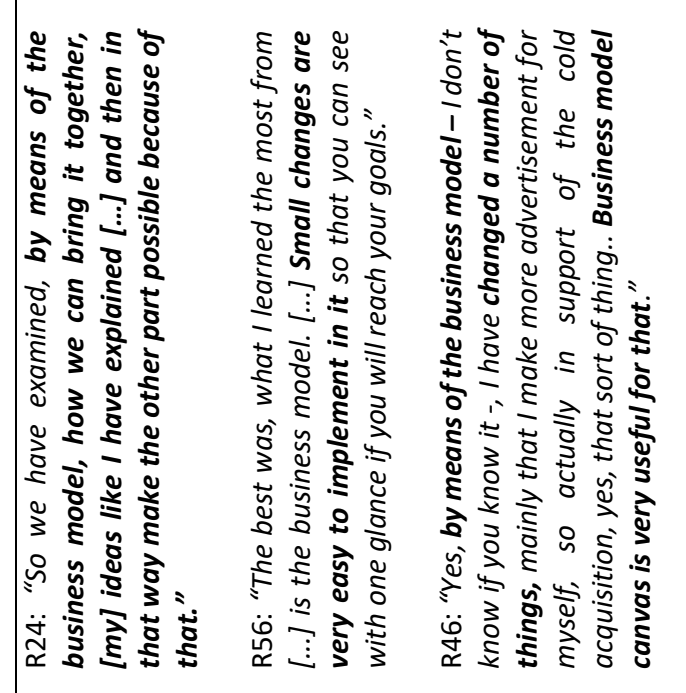

$\stackrel{n}{N}$

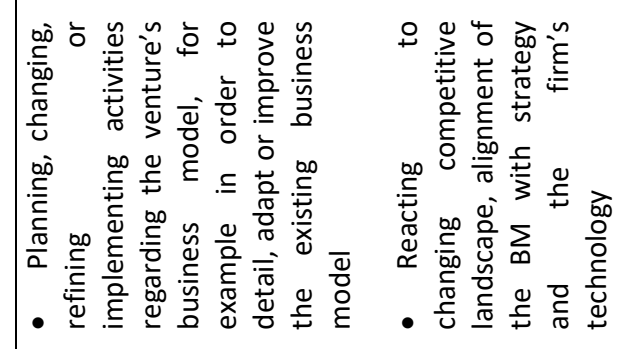

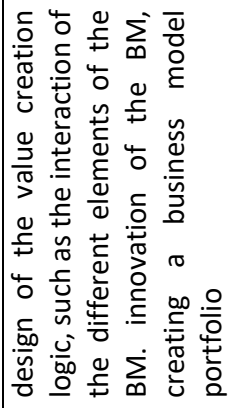

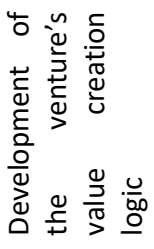




\section{INTER-RATER RELIABILITY}

Inter-rater reliability is an important issue in qualitative research (Silverman, 2001). This can be achieved using researcher triangulation which yields a more empirically informed definition of the theoretical constructs which leads to a more objective way of coding the qualitative data (Flick, 2007). In this study, inter-rater reliability was measured by calculating the percentage agreement and Cohen's kappa. These measures are recognized indicators for inter-rater reliability and have been used in a previous entrepreneurial study carrying out qualitative research data coding. (Grégoire et al., 2010).

Two coders first started with the identification, comparing and contrasting of reports by entrepreneurs in which we identified references to the use of the business model. They independently read four interviews and where they found reference to the business model and its use, for example where respondents described, motivated or reasoned their use of the business model. The coders assigned those (chains of) sentences contained in the quote to the activity or purpose mentioned.

After the coders were finished with four interviews, they compared the coding and they discussed the cases in which their coding result differed and compared how they had assigned a certain code. Based on this, they reached agreement about the interpretation of specific signal words found in the respondents' interviews. They also assessed the reliability of the measuring instrument in terms of relative percentage agreement and Cohen's kappa.

The percentage of agreement between both coders is calculated on the basis of 25 quotes, stemming from 4 interviews. Those quotes are agreed to contain references to business model use. After independent coding, both coders compared their coding outcome. Cohen's kappa was calculated using Brennan \& Prediger's (1981) procedure for the calculation of a kappa when coding more than one nominal category.

Multiple coding of one quote is possible, but does not represent more reliable coding procedures because more categories mathematically affect the calculated kappa, but do not represent a better inter-rater reliability (Bakeman et al., 1997). In our case we distinguish between four categories. In that way, the calculation of Cohen's kappa is more conservative and thus reflects higher robustness. On the basis of a sub-sample of four interviews the calculation of Cohen's kappa yielded an inter-rater reliability of $84 \%$ 
which is considered be an excellent or almost perfect inter-rater reliability (Landis \& Koch, 1977; Fleiss, 1981).

\subsection{RESULTS}

Below we compare the results of the sub-sample which received direct questions about business model use with the sub-sample which did not receive those direct business model questions.

\section{COMPARISON OF THE SUB-SAMPLES BY MEANS OF A T-TEST}

Graph 5-1 shows the number of respondents who report respectively no, one, two, three or four purposes for which they use the business model artifact. As the graph shows, in the sub-sample of respondents who did not receive business model questions there are considerably more entrepreneurs who did not report sensemaking of any purpose. When comparing the two sub-samples on sensemaking of the four purposes, we see that the variance between the sub-samples is relatively small. Reported sensemaking of the different purposes runs in parallel, except for reports of 0 purposes in the two sub-samples.

Graph 5-1 Variance of sensemaking in the two subsamples, in number of purposes mentioned in whole sample and in sub-samples

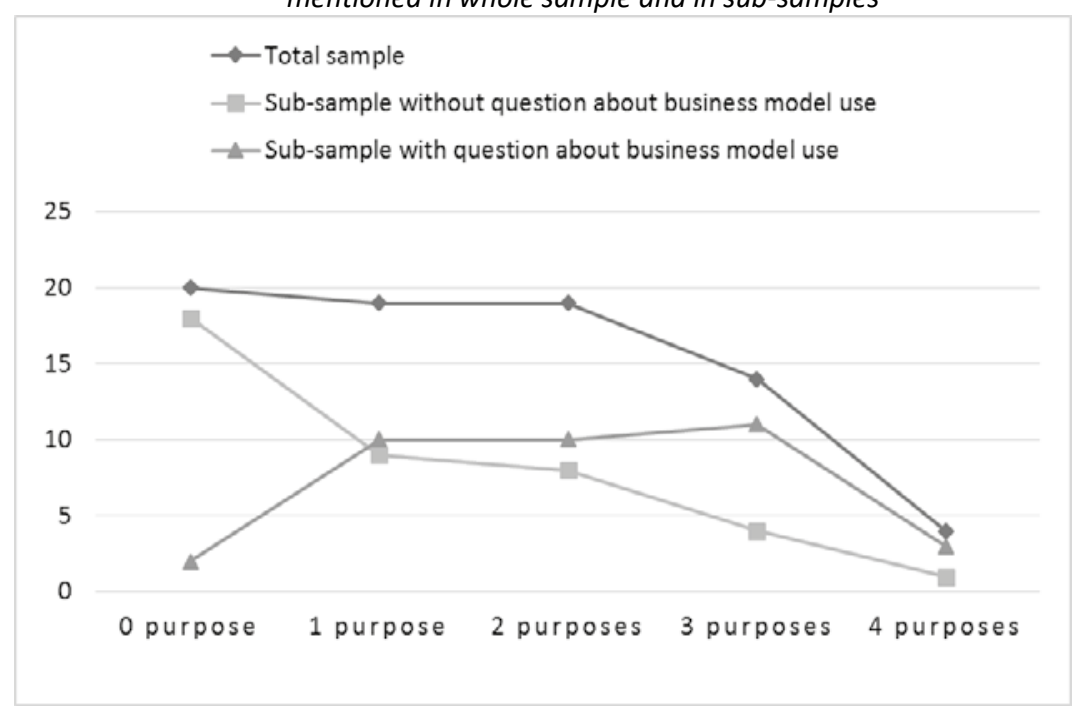


An important point regarding the results shown in graph 5-1 concerns the quantitative differences we see of the report of no purpose for which the business model artifact is used between the two sub-samples. This difference can be explained by the bias of SDR; without targeted questions about the business model, respondents are much less likely to report sensemaking of purposes for which the business model artifact is used and therefore score more on '0 purpose'. In other words: either respondents talk about the use of the business model artifact, or they don't, but when they talk about it there is little difference between the relative amount of number of purposes identified between the sample of entrepreneurs that have been asked direct questions about their use of the business model artifact and the sample which did not receive those questions.

The relative similarity of both sub-samples in terms of number of purposes for which the business model artifact is used by entrepreneurs is reason for us to exclude the respondents who did not report any purposeful use of the business model artifact from further analysis because our focus is not on whether purposes are named or not by entrepreneurs who did receive questions, but on which purposes are named and whether this differs among the two sub-samples. Therefore, to find out whether there is a difference between entrepreneurs in the two sub-samples regarding the average amount of different purposes named, we calculated the variance among the sub-samples minus the entrepreneurs who did not report any purpose $(n=20)$. Of the $n 20$ who did not report any purposes, 16 are entrepreneurs with an educational background in the STEM disciplines.

In order to compare the differences between the results of the entrepreneurs in the two sub-samples regarding the average reporting of one, two, three or even all purposes, we carried out a t-test. A t-test compares the differences between the means of two populations relative to the variability of their scores. Before a t-test can be performed, the samples must be checked for equal variance. To do so, we carried out an f-test with the null hypothesis which presumes that the variances of both subsamples are equal. If F > F Critical one-tail, we can reject the null hypothesis. Table 5-4 shows the outcome of the f-test for variances of scores of the subsample which received direct questions about the business model use and the subsample which did not receive direct questions. 
Table 5-4 F-test two-sample for variances

\begin{tabular}{|l|c|c|}
\hline & $\begin{array}{c}\text { Population which received } \\
\text { direct business model } \\
\text { questions }\end{array}$ & $\begin{array}{c}\text { Population which did not } \\
\text { receive direct business model } \\
\text { questions }\end{array}$ \\
\hline Mean & 2.21 & 1.86 \\
\hline Variance & 0.96 & 0.79 \\
\hline Observations & 34 & 22 \\
\hline df & 33 & 21 \\
\hline $\mathrm{F}$ & 1.21 & \\
\hline $\begin{array}{l}\mathrm{P}(\mathrm{F}<=\mathrm{f}) \text { one- } \\
\text { tail }\end{array}$ & 0.33 & \\
\hline $\begin{array}{l}\mathrm{F} \text { Critical one- } \\
\text { tail }\end{array}$ & 1.99 & \\
\hline
\end{tabular}

The null hypothesis is that in both populations the variance of reported number of purposes is equal. Results of the f-test show that: $F<F$ Critical one-tail. Therefore we confirm the null hypothesis. We also see that the p-value is almost $33 \%$ (with an alpha of $5 \%$ ), which is another indicator that the null hypothesis cannot simply be rejected. We therefore assume relatively equal variances of the scores in the two groups.

Next, we ran a t-test assuming equal variances within the two groups, with the null hypothesis again that the average number of purposes reported in both populations is equal. The results of the t-test are shown in table 5-5.

Table 5-5 T-test assuming equal variances

\begin{tabular}{|l|c|c|}
\hline & $\begin{array}{c}\text { Population which } \\
\text { received direct business } \\
\text { model questions }\end{array}$ & $\begin{array}{c}\text { Population which did not } \\
\text { receive direct business } \\
\text { model questions }\end{array}$ \\
\hline Mean & 2.21 & 1.86 \\
\hline Variance & 0.96 & 0.79 \\
\hline Observations & 34 & 22 \\
\hline $\mathrm{df}$ & 54 & \\
\hline $\mathrm{t}$ Stat & 1.32 & \\
\hline $\mathrm{P}(\mathrm{T}<=\mathrm{t})$ one-tail & 0.095 & \\
\hline $\mathrm{t}$ Critical one-tail & 1.67 & \\
\hline $\mathrm{P}(\mathrm{T}<=\mathrm{t})$ two-tail & 0.19 & \\
\hline $\mathrm{t}$ Critical two-tail & 2.00 & \\
\hline
\end{tabular}

The results of the t-test show that $t$-stat $<\mathrm{t}$ Critical two-tail $(1.32<2.00)$, which means that we cannot reject the null hypothesis. In addition we see that the $p$-value is .09 , indicating that the null hypothesis cannot simply be rejected. This result shows that 
the average number of purposes reported within the two sub-samples does not differ significantly.

The analysis above shows that both subsamples reflect similar pattern of overall distribution. While a comparison between the two populations shows that direct questioning about the use of the business model artifact does lead to a higher count of purposes reported, the results of the t-test show that direct questioning does not affect the relative equality of variance of distribution of scores across the different purposes. In the further results, we therefore discuss the sample as a whole and not the individual subsamples. The results of the whole sample are presented in the next section.

\section{PURPOSES REPORTED BY ENTREPRENEURS}

Respondents use the business model mainly for the purposes of understanding and developing their value creation model. The business model is used for communicative purposes by significantly less respondents, and they hardly report the use of the business model for the purpose of analyzing their value creation logic. Graph 5-2 shows the percentage of respondents of the total sample that reports the use of the business model in terms of the four different purposes.

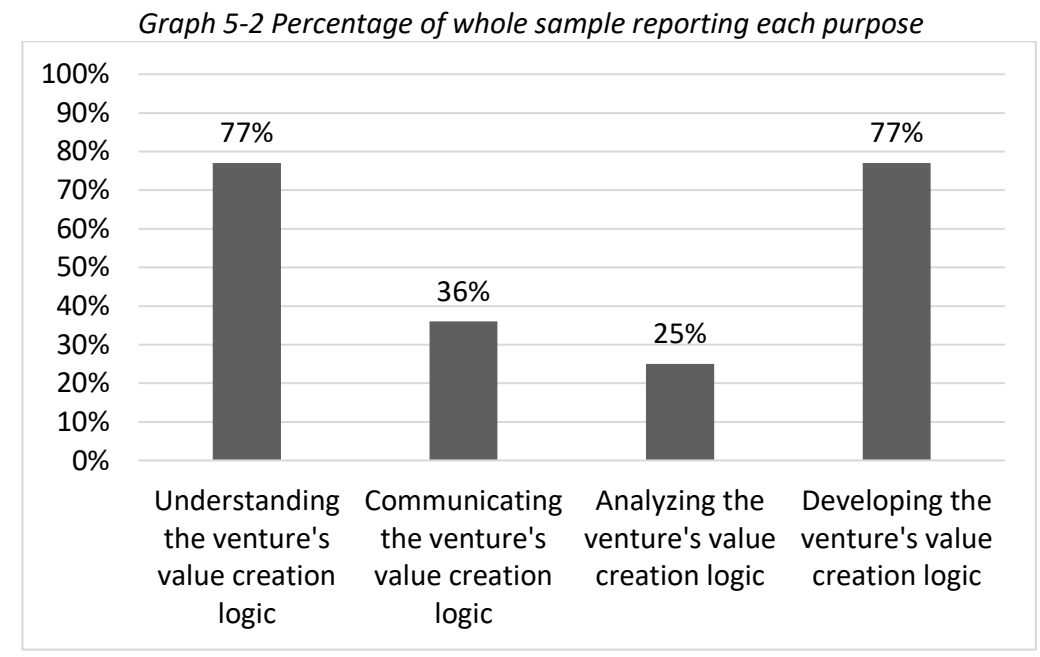

\section{THE PURPOSE OF UNDERSTANDING THE VENTURE'S VALUE CREATION LOGIC}

A little more than three quarters of the respondents in our sample $(77 \%)$ report the use of the business model artifact for the purpose of understanding the venture's value creation logic. For example, they make sense of the venture's business model to gain a comprehensive overview and to delineate the opportunity's focus. The various elements 
of the business model artifact are seen as facilitating the creation of an encompassing, full overview of one's proposed value creation logic, as one respondent describes it:

R05: Yes, nice, a new way of looking at it. [...] I thought it to be quite - it is rather nice because it actually gives an impression of the total concept on one page, yes, that model does that well."

This respondent makes sense of the business model artifact explicitly as a 'model' and this model's usefulness for depicting the total value creation logic of the business. This reflects his use of the business model for comprehensively - yet concisely understanding the venture's value creation logic. Another entrepreneur also emphasizes the comprehensiveness of the business mode artifact. He says:

R08: "Yes, [the business model artifact] I found very enlightening because it gives a total picture. And it not only forces you but gives you the opportunity to formulate and form that total picture. So that there are no more inconsistencies in it."

This entrepreneur appreciates the way in which the business model artifact facilitates the visualization of one's business opportunity which helps to retain a helicopter view instead of 'getting caught up in details'. Other respondents report to use the business model artifact to draw out and define their business idea. Respondents report the business model's facilitating decision-making about the elements of the business idea and their relationship, in the simplest dimensions. In that way, the business model is used to concentrate on a systematic business set-up and thus serves the purpose of understanding value. One respondent describes this as follows:

R17: The business model can be used for a better way of thinking about all the aspects that have to do with the product, customers, service, and suppliers - altogether. If you're very busy you are inclined to think very fragmented: there is an issue and you search for a solution. And because you are often dominated by everyday issues that means that when you are thinking about where you want to go with the venture you often think very opportunistically. With the [...] business model you learn to think about this structurally.

This respondent mentions the inclination to 'think fragmentally' which he associates with 'opportunistic' thinking: namely, everyday issues which yield the danger of 'dominating' his thinking. He juxtaposes this with thinking in a 'structured' way about 'all the aspects' that have to do with a venture, and underlines the role of the venture's business model in facilitating the 'structured' way of thinking about the venture. This 
quote shows that the business model serves as a tool for gaining a systematic overview, which in turn facilitates decision-making about which value the entrepreneur aims to create. Sensemaking of the business model artifact regarding using it for the purpose for understanding the venture's value creation logic reflects the entrepreneurs' intention to keep abreast of all aspects involved in venture development.

\section{THE PURPOSE OF DEVELOPING THE VENTURE'S VALUE CREATION LOGIC}

$77 \%$ of respondents who report sensemaking of the business model artifact in terms of the purpose of managing the venture's value creation logic make sense of this purpose in terms of using it to innovate, change or implement their value creation logic.

Here, sensemaking reflect the entrepreneurs' aim to manage and leverage the business model artifact for the realization of his business idea and related strategy. One entrepreneur for example describes how he has changed his venture's way of value creation, and points out that the business model artifact has helped in this. He speaks about the change he made after a bankruptcy in the following way:

R57: "In first instance, my business model was directed towards product development. [...] I think that actually, in particular with the situation after the bankruptcy, we have examined everything again. Before we were very product-focused, afterwards we had a better balance between product-focus and market-focus, and strongly oriented towards a good cost and revenue structure. That is quite facilitated by the business model."

This respondent describes how the business model facilitates change, which reflects his sensemaking of using the business model for the purpose of developing his venture's value creation logic. The business model's purpose of managing one's value creation model is also used in situations which require strategic re-considerations and choices about changes in the business model, such as a redesign of the value proposition. For example, one entrepreneur uses the business for developmental purposes when talking about the execution of it in the commercial environment:

R28: "Now we have the business model to step by step - focus on execution [...], to document changes in the business model."

The respondent labels the instrumental use of the business model artifact as being able to 'focus on the execution' which reflects his sensemaking in terms of using the business model to carry out strategic activities which relates to the managing purpose. $\mathrm{He}$ also emphasizes the business model artifact as useful for documentation purposes 
which is shared by another entrepreneur. The later mentions the role of the business model artifact in in serving the documentation of experiences:

R24: "It requires continuous attention, a living document, you can put your successive experiences in it."

This entrepreneur describes the business model artifact as a 'living' document' which reflects his sensemaking of the business artifact as able to be changed. This reflects the developmental purpose which the respondent implies. In particular, by adding that he can put 'successive experience' in it, he associates the business model with a tool for documentation of change. Respondents who use the business model for developmental purposes make sense of it in terms of an artifact which facilitates additions to and improvements of the existing value creation model as well as basing their strategic gestation activities on it. Entrepreneurs do so with the motivation of changing their business model in response to external requirements or internal needs.

\section{THE PURPOSE OF COMMUNICATING ABOUT THE VENTURE'S VALUE CREATION LOGIC}

The purpose of communicating value is reported significantly less. $36 \%$ of the entrepreneurs in our sample use the business model for communicative means, such as sharing information with relevant stakeholders. It is perceived to contribute significantly to making one's product or service easily understandable for relevant stakeholders. For example, one entrepreneur says proudly:

R28: "I know now I can create, show my business model to a new audience and get the message across in 15 minutes, I can do that now whenever I need to, that is a good feeling."

This respondent points to the advantages of using the business model to engage in conversation with third parties about one's value creation model. Entrepreneurs in our sample talk about the business model as a means to familiarize people with one's business opportunity. This discourse reflects the entrepreneur's sensemaking of the business model artifact for communicative purposes, in terms of using it to show his venture's value creation logic to 'a new audience' while also pointing out the easiness of communicating that logic by means of this artifact, because it gets the message across in 15 minutes' which is a arguable a rather short time frame. Similarly, another respondent points out: 
R14: "We use [the business model] to get everyone on the same page within the organization, I have presented it to the organization, that is good, then everybody knows what we are doing. And then it's also input for our action plans."

The entrepreneur describes how the venture's business model can be used to create a shared understanding and commitment within the venture for a new business opportunity, which reflects his sensemaking of the business model artifact for a communicative purpose. This reflects the entrepreneur's appreciation of the business model's communicative function not only externally but also internally about the venture's strategy. Another respondent's sensemaking also reflects this 'brainstorming' function of the business model artifact:

R17: "The business model was awesome, a model with which you can brainstorm with everyone, in a graphic way, about the possibilities and choices, about markets, products, you name it, yes, how to make money, with which you can also involve people who are not used to think about these sorts of things, because everyone has an idea."

The quote of this respondent also shows an appreciation of the business model artifact for its quality to 'involve people' by making brainstorming possible in a 'graphic' way. He sees this as a benefit for involving people 'who are not used to think about these sorts of things', and by doing so reaffirms the advantage of the graphic, illustrative, and therefore relatively easy and accessible way of leveraging the business model artifact for the purpose of communication, and to engage others 'because everyone has an idea'. He juxtaposes this to other strategic planning- 'markets, products [..], these sorts of things' - , which are apparently for him perceived as more difficult.

In particular, we found that respondents with a STEM education report the use of the business model artifact for the purpose of communication. This finding will be elaborated in section 4.3.

Sensemaking of the business model artifact for the purpose of communicating the venture's value creation logic is reflected in reports of its use for and in the interaction with various stakeholders, for example when dealing with potential customers, investors, suppliers, but also for internal communication with the managerial team.

\section{THE PURPOSE OF ANALYZING THE VENTURE'S VALUE CREATION}

$25 \%$ of the sample reports the use the business model for the purpose of analyzing their venture's value creation logic. Sensemaking regarding this purpose is reflected in 
the report of activities intended to analyze potential other business models and the development of one's own business model, possibly in comparison to those of competitors. One of those respondents said:

R25: "From what I learned most and what I look at every month, is the business model. That gives me a very good image of where we stand and where we go and if we're on the right track."

The respondent describes how he uses the business model artifact to gain an 'image' of 'if we're on the right track'. He makes sense of the business model artifact in terms of its usefulness for 'checking' the alignment of the venture's business model with what he calls 'the right track'. This goes beyond the purpose of understanding the venture's business model in so far that it involves a 'check' of a previously defined business model, implying a critical analysis of the alignment of the venture's value creation logic. It reflects the entrepreneur's use of the venture's business model for the purpose of analysis. Another respondent also uses the business model artifact for the purpose of analysis and reports his sensemaking of this purpose in the following way:

R02: "I designed about six business models [...]. Before starting the venture I picked the most practical business models to start with. And it ended [...] with a list of criteria of which business models can yield the most profit for me."

While this respondent mentions the design of his business model - thereby referring to the use of the business model artifact for the purpose of understanding the venture's value creation logic - he also talks about a 'list of criteria' and his choosing the business model which would be 'most practical' and 'yield the most profit'. The use of these terms imply a comparison between the various business models - in terms of practicality and profitability - from which he identifies certain criteria that are relevant for him. This way of identifying criteria on the basis of comparison relates to an analytical process, reflecting the entrepreneur's sensemaking of his use of the business model for analytical purposes. Another entrepreneur reports similar sensemaking of the business model when he says:

R18: "My motivation in using the business model was mainly to see if we were on the right track with the present business model."

This respondent emphasizes the use of the business model artifact to see if he was 'on the right track' with his venture's current value creation logic. This implies that he was critically examining whether the way he had organized the businesses' value 
creation logic fits the aim that he had - at some point - set for it. This quote reflects the entrepreneur's use of the business model artifact for analytical purposes.

Sensemaking of the business model artifact for the purpose of analyzing the venture's value creation logic pertains to its enabling nature for critical analysis of the entrepreneur's chosen value creation path, as well as for the comparison with relevant business models of other ventures in the same or similar markets.

THE ROLE OF EDUCATION IN BUSINESS MODEL USE: DIFFERENCES BETWEEN SENSEMAKING OF STEM

\section{AND NON-STEM ENTREPRENEURS}

We now turn to the results of a comparison of respectively respondents with a STEM educational background and those without a STEM educational background. Of the 56 respondents who reported the use of business model artifact for one or more purposes, 28 respondents have a STEM educational background - in science, technology, engineering and mathematics disciplines - and 28 respondents have a nonSTEM educational background in social sciences, liberal arts and humanities. Of the later, half have a background in business administration of economic studies. Graph 53 illustrates the distribution of the amount of purposes mentioned by entrepreneurs (in percentage) within the whole sample and within the two educational sub-samples.

Graph 5-3: Variance in the two educational sub-samples, in terms of number of purposes reported

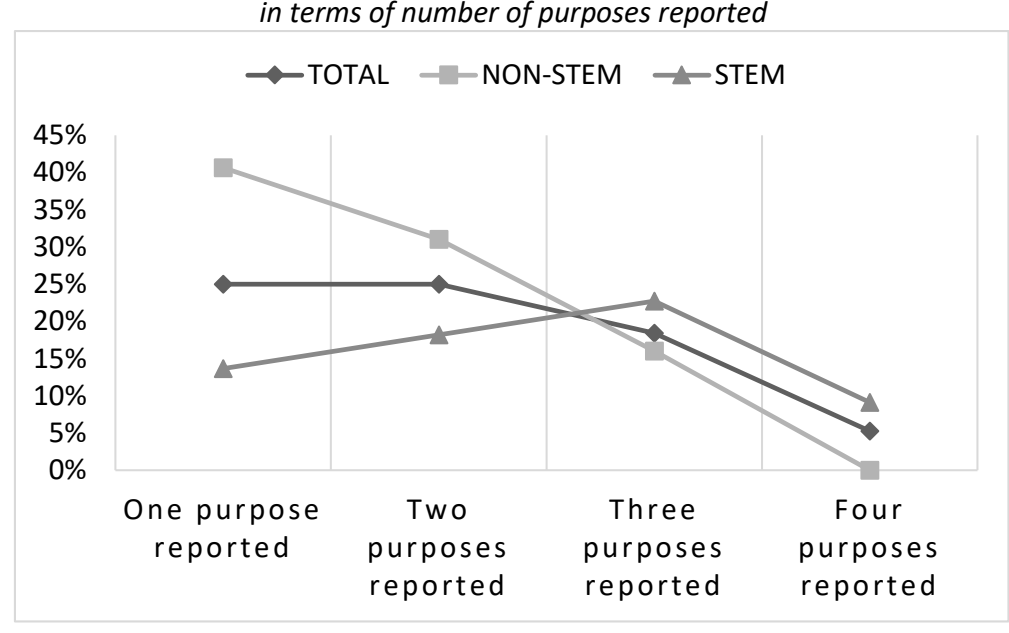

In order to measure the control for the significance of the difference between the populations of those sub-samples, we ran an f-test and a t-test to compare for the variance and the average amount of purposes reported across the two educational subsamples. The null hypothesis is that the both sub-samples are equal regarding the 
average amount of purposes of BM reported. Table 5-6 reports the results of the f-test which shows that $f<F$ Critical which means the sub-samples do not differ significantly regarding their variance.

Tabel 5-6: $f$-test for the educational sub-samples

\begin{tabular}{|l|c|c|}
\hline & STEM & non-STEM \\
\hline Mean & 2.43 & 1.71 \\
\hline Variance & 0.99 & 0.58 \\
\hline Observations & 28 & 28 \\
\hline df & 27 & 27 \\
\hline $\mathrm{F}$ & 1.71 & \\
\hline $\begin{array}{l}\mathrm{P}(\mathrm{F}<=\mathrm{f}) \text { one- } \\
\text { tail }\end{array}$ & 0.09 & \\
\hline $\begin{array}{l}\text { F Critical one- } \\
\text { tail }\end{array}$ & 1.90 & \\
\hline
\end{tabular}

Next, we carried out a t-test assuming equal variances within the two sub-samples. Table 5-7 displays the outcome of the t-test which we conducted only using the respondents who reported the use of purposes.

Table 5-7 t-Test for two-sample assuming equal variances

\begin{tabular}{|l|r|r|}
\hline & \multicolumn{1}{c|}{ STEM } & \multicolumn{1}{c|}{ non-STEM } \\
\hline Mean & 2.428571 & 1.714286 \\
\hline Variance & 0.994709 & 0.582011 \\
\hline Observations & 28 & 28 \\
\hline$d f$ & 54 & \\
\hline$t$ Stat & 3.01005 & \\
\hline $\mathrm{P}(\mathrm{T}<=\mathrm{t})$ one-tail & 0.001983 & \\
\hline $\mathrm{t}$ Critical one-tail & 1.673565 & \\
\hline $\mathrm{P}(\mathrm{T}<=\mathrm{t})$ two-tail & 0.003965 & \\
\hline $\mathrm{t}$ Critical two-tail & 2.004879 & \\
\hline
\end{tabular}

The results of the statistical test presented in table 5-6 show that $\mathrm{t}$-stat $>\mathrm{t}$ Critical two tail $(3.01>2.00)$. In addition, the $p$-value is very small $(0.2 \%)$. Based on this result we can reject the null hypothesis; the difference between the two samples is significant. Entrepreneurs with a background in STEM disciplines report the use of the business model artifact for more purposes significantly more often than entrepreneurs with a nonSTEM educational background.

In particular, there are notable differences in the reported use of the communicative and the analytical purpose. In graph 5-4 we compare the relative use of purposes by entrepreneurs in the two sub-samples who had been asked explicitly about their use of 
the business model artifact. As the graph shows, almost $60 \%$ of STEM entrepreneurs who received business model questions reported sensemaking of the business model artifact in terms of its communicative purpose, compared to only $19 \%$ of the non-STEM entrepreneurs who received those questions. We also see that $20 \%$ of the STEM entrepreneurs report the use of the business model artifact for its analytical purpose, compared to only $6 \%$ of the non-STEM entrepreneurs.

Graph 5-4: Comparison of reported purposes by entrepreneurs who received business model questions per educational sub-sample

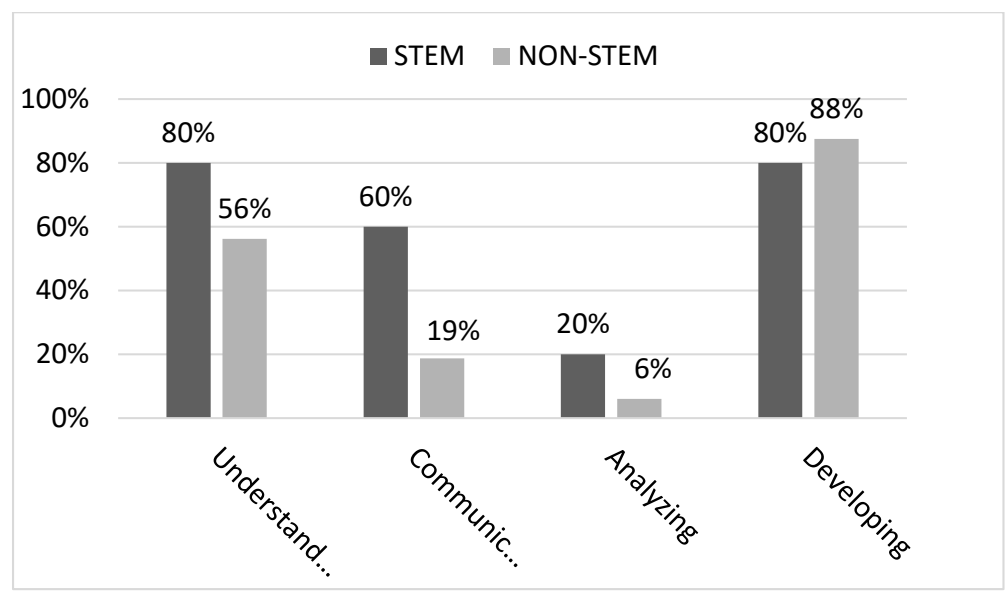

The comparison between entrepreneurs with a background in STEM disciplines (from here onwards named 'STEM entrepreneurs') and those with a background in nonSTEM disciplines (from here onwards named 'non-STEM entrepreneurs') shows that both populations most often use the purposes of understanding and developing the venture's value creation logic.

This comparison also yields two important differences between STEM and nonSTEM entrepreneurs. One relates to the amount of reported purposes identified in the respondents' sensemaking, while the other relates to which purposes are reported by STEM and non-STEM entrepreneurs. We elaborate on each of these differences below.

In graph 5-3 is shown that the amount of purposes reported sensemaking differs between STEM and non-STEM entrepreneurs. STEM entrepreneurs exhibit a more 'extreme' use of the purposes of the business model artifact insofar that there are two distinct groups of STEM entrepreneurs. 
One group of STEM entrepreneurs makes much less sense of the business model artifact, which is illustrated by a lower number of STEM entrepreneurs who report the use of one or two purposes in comparison to non-STEM entrepreneurs. As we recall, STEM entrepreneurs also don't report any purposeful use of the business model artifact four times more than non-STEM entrepreneurs. The other group of STEM entrepreneurs uses the business model artifact for more purposes than non-STEM entrepreneurs. This is demonstrated by the fact that STEM respondents report sensemaking of their use of the business model artifact for more than two purposes more often than non-STEM entrepreneurs.

This result shows that the STEM entrepreneurs are likely to either report no or less purposeful use of the business model artifact, or they report the use of more purposes of the business model artifact than non-STEM entrepreneurs.

The second difference between STEM and non-STEM entrepreneurs is linked to the purposes for which the business model artifact is used. Graph 5-4 shows that the STEM entrepreneurs make sense of using the business model artifact more often than the non-STEM entrepreneurs for three of the four purposes: the purposes of understanding, communicating and analyzing the venture's value creation logic. In particular, the latter two purposes are identified three times as much in the reported sensemaking of STEM entrepreneurs.

These two differences between STEM and non-STEM entrepreneurs show that STEM entrepreneurs are more extremely distributed in terms of their use of the business model artifact; but when they use the business model artifact, they use it for more purposes than non-STEM entrepreneurs.

\subsection{Conclusion AND Discussion}

In this study we examined how entrepreneurs use the business model during opportunity development. To do so, we analyze how entrepreneurs make sense of their purposeful use of the business model artifact. While existing literature has identified different purposes of the venture's business model for opportunity development, our study is the first to empirically, systematically and comparatively show how those purposes are used in practice during opportunity development. In this section, we 
present our conclusions in more detail and discuss how they contribute to the existing literature.

\section{SENSEMAKING OF THE BUSINESS MODEL ARTIFACT PURPOSES}

Based on our findings we conclude that sensemaking of the business model artifact by entrepreneurs mainly relates to using it for purposes of understanding and developing the venture's value creation logic. To a significantly lesser extent, entrepreneurs also make sense of the business model artifact by using it for the purposes of communicating and analyzing their venture's value creation logic. The dominance of sensemaking in terms of purposes of understanding and developing the venture's value creation logic fits with cognitive processes linked to opportunity development which these entrepreneurs are engaged in.

As we saw in the results of our analysis, entrepreneurs report using the business model artifact's purpose of analyzing the venture's value creation logic the least of all purposes. The lack of making sense of this purpose of the business model artifact is worth noting in the light of Martins et al.'s (2015) study. Finding a positive relationship between the analytical exploration and the effectiveness of business model innovation, the authors underline that key to effective development of the venture's business model is the analysis of the existing business model in comparison to the target business model. While these authors thus emphasize the positive impact of using the venture's business model for analytical purposes, our findings suggest that entrepreneurs do not use this purpose.

In addition, an important result relates to the difference between the sensemaking of STEM and non-STEM entrepreneurs regarding the use the business model artifact. STEM entrepreneur in our sample are four times more likely to not report using the business model artifact purposefully at all, but when they report it, they report using the business model artifact for two or more purposes more often than non-STEM entrepreneurs. The reason for this extreme distribution could be that in the case of STEM entrepreneurs who don't report the use of the business model artifact their product or service-offering is already clarified and far advanced in terms of commercially viable, and is therefore less prone to be used for one of the four purposes.

Another explanation for this result is associated with the entrepreneurs' selfperception. Douglas (2009) wrote about the perceptions of entrepreneurs and pointed out that entrepreneurs tend to see themselves as more competent than non- 
entrepreneurs see themselves. This would explain why the large number of entrepreneurs in the STEM population are unable to see the benefits of purposefully using their venture's business model: they can be seen as the type of entrepreneur which is seen as 'unknowingly incompetent'; while the business model artifact could certainly be of use to them - if only to critically analyze their value creation logic, if not for anything else - they feel that they are competent enough to judge its use as not necessary. On the other side of the extreme, we find STEM entrepreneurs who more often report the use of more purposes, and in particular use the business model artifact's purposes of communicating and analyzing significantly more often than the non-STEM entrepreneurs; on this extreme of the scale they are in fact 'knowingly and competently' using the business model artifact.

\section{THE BUSINESS MODEL ARTIFACT AS SOCIALLY SITUATED COGNITIVE MECHANISM}

The second conclusion relates to entrepreneurial sensemaking of the business model artifact for the purpose of communicating the venture's value creation logic. Our findings show that STEM entrepreneurs are three times more likely to use the business model artifact for the communicating purpose than non-STEM entrepreneurs.

This result can be explained by the STEM entrepreneurs' 'entrepreneurial type' as described in existing entrepreneurship literature. Research shows that entrepreneurs with an (exclusively) technical education - such as the STEM disciplines - are less likely to effectively communicate orally and written than entrepreneurs with a (partially) nontechnical background (Smith, 1967; Smith \& Miner, 1983). Therefore STEM entrepreneurs are less likely to report the purposeful use of the business model artifact - they are simply not very able to or interested in communicating about the use of the business model artifact. Yet, if and when they communicate about it, they are more likely than non-STEM entrepreneurs to use it for communicating purposes because they have a higher need for a communication tool than the non-STEM entrepreneurs or at least make more often sense of the business model artifact accordingly. This instrumental use suits their dispositional needs indicated by existing research on this type of entrepreneur (Smith, 1967).

Our results show that STEM entrepreneurs make sense of the business model artifact in terms of an instrument to engage stakeholders in communication about the venture, in order to receive their feedback and advice. By enabling the input of stakeholders in entrepreneurial decision-making about and during opportunity 
development, the business model artifacts functions as mechanism for realizing interactive, interpretative decision-making processes during value creation.

This use of the business model artifact can be explained with the socially situated cognitive perspective on entrepreneurship. From the perspective of socially situated cognition, language is paramount for shared sensemaking activities in entrepreneurship (Clarke \& Cornelissen, 2011). Cornelissen (2014) posit that language shapes entrepreneurial cognition by providing a means to verbalize one's ideas and "shape or limit the ways in which the speaker forms conceptions of the world" (Clarke \& Cornelissen, 2014: p. 387). Business opportunities are seen to emerge in the social context "of speaking and interactions with others" which affect "the construction of meaning about a new venture" (Cornelissen \& Clarke, 2010: p. 542). The authors suggest that language plays an important role in the successful development of a business opportunity; a similar observation was made by Zott \& Huy (2007). Using the business model artifact for its communicative purpose enables entrepreneurs to express their business idea to other parties verbally.

We conclude that the communicating purpose of the business model provides a means for STEM entrepreneurs to move from what Berry (1996) called 'technical mindset' to a more 'value-oriented' mindset. It enables the input of those stakeholders in entrepreneurial decision-making about the venture's value creation logic and in doing so functions as a mechanism for shared sensemaking with the aim of value co-creation. Used for its communicating purpose, the business model artifact thus facilitates the entrepreneur's focus on customer value creation by enabling the engagement of relevant network partners required for that value (co-)creation.

\section{PRACTICAL IMPLICATIONS OF THIS STUDY}

As of yet, studies into the practical employment of the business model artifact during opportunity development are sparse (George \& Bock, 2011). The conflict between the role accorded to lean startup, flexibility and improvisation on the one hand, and entrepreneurial need for direction on the other hand yields cause for research on this matter. The results of this study have implications for entrepreneurs and for the set-up of business incubation programs.

For entrepreneurs, the advantages of developing their awareness for the different purposes for which the business model artifact can be used are evident: In learning to 
use them, they can become more knowingly competent about leveraging their venture's value creation logic during opportunity development. In generally, using the analytical and communicative purposes more can help to critically reflect and receive feedback on the different aspects of the venture. The use of the business model for its analytical purpose could benefit the effectiveness of a business model innovation just like Martins et al. (2015) propose. And the use of the communicating purpose facilitates the engagement with and reception of input from relevant stakeholders during business opportunity identification and preparation which enhances opportunities which facilitate the legitimizing process described as relevant for successful opportunity development (Zott \& Huy, 2007). This is in particular relevant for STEM entrepreneurs who can leverage the communicating purpose of the business model artifact to move from a product-oriented to a more value-oriented mindset.

By raising awareness for the various purposes for which the business artifact can be used, ensuing entrepreneurial sensemaking of these purposes and their subsequent effective use could contribute successful business model change or innovation, and eventually to successful venture development.

Also, the findings of this study allow practitioners to better align (institutional) support efforts in business planning, such as focused training in order to raise awareness for the different purposes of the business model artifact and to encourage the using the business model artifact for them. For example, as stated above, we found that the use of the business model's purposes of communicating and analyzing the venture's value creation logic are less reported by entrepreneurs - in particular by the non-STEM entrepreneurs - than the other two purposes. We argue that raising the awareness for the analyzing purpose of the business model artifact by means of trainings or workshops can facilitate business model development since research shows that there is a positive relationship between an analytical exploration of the venture's existing business model and the effectiveness of business model innovation (Martins et al., 2015). And training in using the business model for communicative purposes can help to engage relevant stakeholders in the entrepreneur's 'community of inquiry' (Shepherd \& Patzelt, 2017) which is relevant for providing further feedback and shaping of the business opportunity.

To make institutional training programs targeted at making venture development more efficient, policy-makers can raise awareness among entrepreneurs for the business model's purposes of communicating and analyzing the venture's value creation 
logic. We propose that a better understanding of the different purposes is conducive to using them more extensively, which ultimately could contribute to more successful business model innovation.

\subsection{LIMITATIONS AND FUTURE RESEARCH}

There are a number of limitations to our study. Most importantly, we drew on a selfselected sample of entrepreneurs who participated in a business incubation/acceleration program, and therefore cannot be treated as representative. Due to the research setting, we cannot provide a comparison with entrepreneurs outside the incubation/acceleration setting. We are therefore unable to tell whether entrepreneurs in our sample use the business model differently than entrepreneurs outside an institutional support setting. On a related note, we were also unable to control for phase of business opportunity development; it is possible that those respondents who are much further in terms of gestation activities mention more functions since they are able to use more of the business model purposes, for example make more use of the communicative or analyzing purpose. Still, it remains surprising that respondents hardly use the business model for the purpose of critically analyzing and comparing their business model, considering that they had received information about the various purposes of their business model during the incubation/acceleration program.

Another limitation is that of the use of interviews. Our data captured the ex post perception of the use of business model functions, and might suffer from lack of or biases in the memories that respondents reported to us in the interviews. Unfortunately realtime data was not available for the purposes of our study.

Based on our results, we also identify the following research alleys.

Future research on the benefits of the various functions and how to stimulate the use of them, can yield valuable insights into mechanisms which facilitate value creation processes. Foss et al. (2008), Kraaijenbrink et al. (2010) and Rasmussen (2011) for example emphasize the importance of a closer analysis of micro-level processes and phenomena regarding entrepreneurs. We suggest that research into the individual reasons for under-utilizing the purpose of analyzing value could yield interesting insights into the relationship between the use of the analytical purpose and the identification of 
what Martin et al. (2015) call prototypical attributes, and subsequent effective development of innovation of the venture's business model.

On a related note, investigating why some entrepreneurs do not use the business model artifact for any purposes, as we found in the case of a substantial number of entrepreneurs with a background in STEM disciplines, would yield valuable results about the motivations and argumentations of entrepreneurs to use or not to use the business model artifacts and to what extent this motivation or argumentation affects their openness to changing their business model or the effect of their use or non-use of the business model artifact on subsequent opportunity development, venture survival and growth. An analysis of a larger group of entrepreneurs with different background might be more effective in the identification of subtle differences in their sensemaking of the purposes for which the business model artifact can be used. That is because a larger sample offers the opportunity to examine whether the slight differences between the perceptions of entrepreneurs regarding the leverage of different business model purposes become aggravated or instead disappear altogether.

Furthermore, more research on the effect of human capital on utilization of business planning instruments can help us identify important variables in the way entrepreneurs use planning instruments, and which variables affect successful use of those instruments. For example, the effects of previous employment, team composition, or individual background variables - such as parental entrepreneurship experience, phase of business development, or serial entrepreneurship experience - can contribute to a more detailed understanding of cognitive and socio-cultural influences on business planning during venture development. We full-heartedly join in the call of Haynie et al. (2009) for more detailed analysis of the influence of human capital variables on business opportunity identification and preparation, in order to offer more detailed insights into the mechanisms which contribute to successful venture development, survival and growth.

\subsection{BIBLIOGRAPHY}

Al-Debei, M. M. and D. Avison (2010). Developing a unified framework of the business model concept. European Journal of Information Systems 19(3): 359-376. 
Arend, R. J. (2013). The business model: Present and future-beyond a skeumorph. Strategic Organization, 11(4), 390-402.

Baden-Fuller, C., \& Morgan, M. S. (2010). Business models as models. Long range planning, 43(2), 156-171.

Bakeman, R., McArthur, D., Quera, V., \& Robinson, B. F. (1997). Detecting sequential patterns and determining their reliability with fallible observers. Psychological Methods, 2(4), 357.

Baron, R. A., \& Ensley, M. D. (2006). Opportunity recognition as the detection of meaningful patterns: Evidence from comparisons of novice and experienced entrepreneurs. Management science, 52(9), 1331-1344.

Berry, M. M. (1996). Technical entrepreneurship, strategic awareness and corporate transformation in small high-tech firms. Technovation, 16(9), 487-522.

Bettiol, M., Di Maria, E., \& Finotto, V. (2012). Marketing in SMEs: the role of entrepreneurial sensemaking. International Entrepreneurship and Management Journal, 8(2), 223-248.

Bird, B. (2014). Toward a taxonomy of entrepreneurs' behavior. Handbook of Entrepreneurial Cognition, 113-131.

Blank, S. G. (2005). The Four Steps to the Epiphany. Cafepress.com, 2005.

Brennan, R. L., \& Prediger, D. J. (1981). Coefficient kappa: Some uses, misuses, and alternatives. Educational and psychological measurement, 41(3), 687-699.

Brinckmann, J., \& Kim, S. M. (2015). Why we plan: the impact of nascent entrepreneurs' cognitive characteristics and human capital on business planning. Strategic Entrepreneurship Journal, 9(2), 153-166.

Chesbrough, H. and R. S. Rosenbloom (2002). "The role of the business model in capturing value from innovation: evidence from Xerox Corporation's technology spin-off companies." Industrial and Corporate Change 11(3): 529-555.

Chesbrough, H. (2010). Business model innovation: opportunities and barriers. Long range planning, 43(2), 354-363.

Clarke, J. (2011). Revitalizing entrepreneurship: how visual symbols are used in entrepreneurial performances. Journal of Management Studies, 48(6), 1365-1391. 
Clarke, J., \& Cornelissen, J. (2011). Language, communication, and socially situated cognition in entrepreneurship. Academy of Management Review, 36(4), 776-778.

Clarke, J. S., \& Cornelissen, J. P. (2014). 11. How language shapes thought: new vistas for entrepreneurship research. Handbook of Entrepreneurial Cognition, 383.Davidsson, P. (1988). Type of man and type of company revisited: a confirmatory cluster analysis approach. Frontiers of Entrepreneurship research, 88-105.

Cornelissen, J. P., \& Clarke, J. S. (2010). Imagining and rationalizing opportunities: inductive reasoning and the creation and justification of new ventures. Academy of Management Review, 35(4), 539-557.

Cornelissen, J. P., Mantere, S., \& Vaara, E. (2014). The contraction of meaning: The combined effect of communication, emotions, and materiality on sensemaking in the Stockwell shooting. Journal of Management Studies, 51(5), 699-736.

Davidsson, P. and B. Honig (2003). "The role of social and human capital among nascent entrepreneurs." Journal of Business Venturing 18(3): 301-331.

Delmar, F., \& Shane, S. (2003). Does business planning facilitate the development of new ventures?. Strategic management journal, 24(12), 1165-1185.

Demil, B., \& Lecocq, X. (2010). Business model evolution: in search of dynamic consistency. Long range planning, 43(2), 227-246.

Demil, B., Lecocq, X., Ricart, J. E., \& Zott, C. (2015). Introduction to the SEJ special issue on business models: business models within the domain of strategic entrepreneurship. Strategic Entrepreneurship Journal, 9(1), 1-11.

Doganova, L., \& Eyquem-Renault, M. (2009). What do business models do?: Innovation devices in technology entrepreneurship. Research Policy, 38(10), 1559-1570.

Douglas, E. (2009). Perceptions-Looking at the world through entrepreneurial lenses. In Understanding the entrepreneurial mind (pp. 3-22). Springer New York.

Downing, S. (2005). The social construction of entrepreneurship: Narrative and dramatic processes in the coproduction of organizations and identities. Entrepreneurship theory and Practice, 29(2), 185-204. 
Ensley, M. D., \& Hmieleski, K. M. (2005). A comparative study of new venture top management team composition, dynamics and performance between university-based and independent start-ups. Research Policy, 34(7), 1091-1105.

Fleiss, J.L. (1981). Statistical methods for rates and proportions (2nd ed.). New York: John Wiley.

Flick, U. (2007). "Managing Quality in Qualitative Research." Sage: London, Thousand Oaks, New Delhi.

Foss, N. J., P. G. Klein, et al. (2008). "Entrepreneurship, subjectivism, and the resourcebased view: toward a new synthesis." Strategic Entrepreneurship Journal 2(1): 73-94.

Franklin, S. J., Wright, M., \& Lockett, A. (2001). Academic and surrogate entrepreneurs in university spin-out companies. The Journal of Technology Transfer, 26(1-2), 127-141.

Gambardella, A., \& McGahan, A. M. (2010). Business-model innovation: General purpose technologies and their implications for industry structure. Long range planning, 43(2), 262-271.

Garud, R., \& Giuliani, A. P. (2013). A narrative perspective on entrepreneurial opportunities. Academy of Management Review, 38(1), 157-160.

George, G., \& Bock, A. J. (2011). The business model in practice and its implications for entrepreneurship research. Entrepreneurship theory and practice, 35(1), 83-111.

Gerasymenko, V., De Clercq, D., \& Sapienza, H. J. (2015). Changing the business model: Effects of venture capital firms and outside CEOs on portfolio company performance. Strategic Entrepreneurship Journal, 9(1), 79-98.

Gioia, D. A., \& Chittipeddi, K. (1991). Sensemaking and sensegiving in strategic change initiation. Strategic management journal, 12(6), 433-448.

Gioia, D. A., Thomas, J. B., Clark, S. M., \& Chittipeddi, K. (1994). Symbolism and strategic change in academia: The dynamics of sensemaking and influence. Organization science, 5(3), 363-383.

Grégoire, D. A., Barr, P. S., \& Shepherd, D. A. (2010). Cognitive processes of opportunity recognition: The role of structural alignment. Organization Science, 21(2), 413-431. 
Gupta, V., MacMillan, I. C., \& Surie, G. (2004). Entrepreneurial leadership: developing and measuring a cross-cultural construct. Journal of Business Venturing, 19(2), 241260.

Haynie, J. M., Shepherd, D. A., \& McMullen, J. S. (2009). An opportunity for me? The role of resources in opportunity evaluation decisions. Journal of Management studies, 46(3), 337-361.

Hill, R. C., \& Levenhagen, M. (1995). Metaphors and mental models: Sensemaking and sensegiving in innovative and entrepreneurial activities.Journal of Management, 21(6), 1057-1074.

Hmieleski, K. M., \& Baron, R. A. (2009). Entrepreneurs' optimism and new venture performance: A social cognitive perspective. Academy of management Journal, 52(3), 473-488.

Holt, R., \& Macpherson, A. (2010). Sensemaking, rhetoric and the socially competent entrepreneur. International Small Business Journal, 28(1), 20-42.

Humphreys, M., Ucbasaran, D., \& Lockett, A. (2012). Sensemaking and sensegiving stories of jazz leadership. Human Relations, 65(1), 41-62.

Knight, F. H. (1921). Risk, uncertainty and profit. New York: Hart, Schaffner and Marx.

Knockaert, M., Clarysse, B., \& Wright, M. (2010). The extent and nature of heterogeneity of venture capital selection behaviour in new technology-based firms. $R \& d$ Management, 40(4), 357-371.

Kraaijenbrink, J., Spender, J. C., \& Groen, A. J. (2010). The resource-based view: a review and assessment of its critiques. Journal of management, 36(1), 349-372.

Krueger Jr, N. F., \& Day, M. (2010). Looking forward, looking backward: From entrepreneurial cognition to neuroentrepreneurship. In Handbook of entrepreneurship research (pp. 321-357). Springer New York.

Lambert, S. C., \& Davidson, R. A. (2013). Applications of the business model in studies of enterprise success, innovation and classification: An analysis of empirical research from 1996 to 2010. European Management Journal, 31(6), 668-681.

Landis, J. R., \& Koch, G. G. (1977). The measurement of observer agreement for categorical data. Biometrics, 159-174. 
Magretta, J. (2002). "Why business models matter." Harvard Business Review 80(5): 8693.

Maitlis, S., \& Sonenshein, S. (2010). Sensemaking in crisis and change: Inspiration and insights from Weick (1988). Journal of management studies,47(3), 551-580.

Maitlis, S., \& Christianson, M. (2014). Sensemaking in organizations: Taking stock and moving forward. The Academy of Management Annals, 8(1), 57-125.

Martins, L. L., Rindova, V. P., \& Greenbaum, B. E. (2015). Unlocking the hidden value of concepts: a cognitive approach to business model innovation.Strategic Entrepreneurship Journal, 9(1), 99-117.

Mason, K., \& Spring, M. (2011). The sites and practices of business models. Industrial Marketing Management, 40(6), 1032-1041.

McMullen, J. S., \& Shepherd, D. A. (2006). Entrepreneurial action and the role of uncertainty in the theory of the entrepreneur. Academy of Management review, 31(1), 132-152.

Mitchell, R. K., Randolph-Seng, B., \& Mitchell, J. R. (2011). Socially situated cognition: Imagining new opportunities for entrepreneurship research.Academy of Management Review, 36(4), 774-776.

Navis, C., \& Glynn, M. A. (2010). How new market categories emerge: Temporal dynamics of legitimacy, identity, and entrepreneurship in satellite radio, 19902005. Administrative Science Quarterly, 55(3), 439-471.

Navis, C., \& Glynn, M. A. (2011). Legitimate distinctiveness and the entrepreneurial identity: Influence on investor judgments of new venture plausibility. Academy of Management Review, 36(3), 479-499.

Nenonen, S., \& Storbacka, K. (2010). Business model design: conceptualizing networked value co-creation. International Journal of Quality and Service Sciences, 2(1), 43-59.

Organisation for Economic Cooperation and Development. (2014). Education at a Glance 2014: OECD Indicators. OECD Publishing, Paris. 
Osiyevskyy, O., \& Dewald, J. (2015). Explorative Versus Exploitative Business Model Change: The Cognitive Antecedents of Firm-Level Responses to Disruptive Innovation. Strategic Entrepreneurship Journal,9(1), 58-78.

Osterwalder, A. (2004). The business model ontology: A proposition in a design science approach.

Osterwalder, A., Pigneur, Y., \& Tucci, C. L. (2005). Clarifying business models: Origins, present, and future of the concept. Communications of the association for Information Systems, 16(1), 1.

Paulhus, D. L. (2002). Socially desirable responding: The evolution of a construct. The role of constructs in psychological and educational measurement, 49-69.

Rasmussen, E., S. Mosey, et al. (2011). "The Evolution of Entrepreneurial Competencies: A Longitudinal Study of University Spin-Off Venture Emergence." Journal of Management Studies 48(6): 1314-1345.

Rosenberg, M. J. (1965). When dissonance fails: On eliminating evaluation apprehension from attitude measurement. Journal of personality and social psychology, 1(1), 28.

Sarasvathy, S. D. (2001). Causation and effectuation: Toward a theoretical shift from economic inevitability to entrepreneurial contingency. Academy of management Review, 26(2), 243-263

Schindehutte, M., Morris, M. H., \& Kocak, A. (2008). Understanding market-driving behavior: the role of entrepreneurship. Journal of Small Business Management, 46(1), 4-26.

Schwandt, D. R. (2005). When managers become philosophers: Integrating learning with sensemaking. Academy of Management Learning \& Education,4(2), 176-192.

Selden, P. D., \& Fletcher, D. E. (2015). The entrepreneurial journey as an emergent hierarchical system of artifact-creating processes. Journal of Business Venturing, 30(4), 603-615.

Shaver, K. G., \& Scott, L. R. (1991). Person, process, choice: The psychology of new venture creation. Entrepreneurship theory and practice, 16(2), 23-45. 
Shepherd, D. A., \& Patzelt, H. (2017). Researching the Generation, Refinement, and Exploitation of Potential Opportunities. In Trailblazing in Entrepreneurship (pp. 17-62). Springer International Publishing.

Silverman, D. (2001). "Interpreting qualitative data: methods for analyzing talk, text, and interaction." Sage Publications: London, Thousand Oaks, New Delhi.

Smith, N. R. (1967). The entrepreneur and his firm: The relationship between type of man and type of company. Occasional Papers, Bureau of Business and Economic Research, Michigan State University, 109.

Smith, N., \& Miner, J. (1983). Type of Entrepreneur, Type of Firm, and Managerial Motivation: Implications for Organizational Life Cycle Theory. Strategic Management Journal, 4(4), 325-340.

Sosna, M., Trevinyo-Rodríguez, R. N., \& Velamuri, S. R. (2010). Business model innovation through trial-and-error learning: The Naturhouse case. Long range planning, 43(2), 383-407.

Spieth, P., Schneckenberg, D., \& Ricart, J. E. (2014). Business model innovation-state of the art and future challenges for the field. R\&D Management, 44(3), 237-247.

Stigliani, I., \& Ravasi, D. (2012). Organizing thoughts and connecting brains: Material practices and the transition from individual to group-level prospective sensemaking. Academy of Management Journal, 55(5), 1232-1259.

Teece, D. J. (2010). "Business Models, Business Strategy and Innovation." Long Range Planning 43(2-3): 172-194.

Timmers, P. (1998). Business models for electronic markets. Electronic markets 8(2): 38.

Tonkiss, F. (1998). Continuity/change. Core sociological dichotomies. London: Sage, 3448.

Trimi, S., \& Berbegal-Mirabent, J. (2012). Business model innovation in entrepreneurship. International Entrepreneurship and Management Journal,8(4), 449465.

Venkataraman, S., Sarasvathy, S. D., Dew, N., \& Forster, W. R. (2013). Of narratives and artifacts. Academy of Management Review, 38(1), 163-166. 
Weick, K. E. (1995). Sensemaking in organizations (Vol. 3). Sage.

Weick, K. E., Sutcliffe, K. M., \& Obstfeld, D. (2005). Organizing and the process of sensemaking. Organization science, 16(4), 409-421.

Weick, K. E. (2012). Organized sensemaking: A commentary on processes of interpretive work. Human Relations, 65(1), 141-153.

van Werven, R., Bouwmeester, O., \& Cornelissen, J. P. (2015). The power of arguments: How entrepreneurs convince stakeholders of the legitimate distinctiveness of their ventures. Journal of Business Venturing, 30(4), 616-631.

West, G. P., \& Noel, T. W. (2009). The impact of knowledge resources on new venture performance. Journal of Small Business Management, 47(1), 1-22.

Zott, C., \& Huy, Q. N. (2007). How entrepreneurs use symbolic management to acquire resources. Administrative Science Quarterly, 52(1), 70-105.

Zott, C., Amit, R., \& Massa, L. (2011). The business model: recent developments and future research. Journal of management, 37(4), 1019-1042. 
Chapter 6

Conclusion 


\subsection{RESEARCH PROBLEM AND RESEARCH QUESTION}

Central to this thesis is the topic of entrepreneurial cognitive development during business opportunity development. Entrepreneurial cognitive processes are shaped in interaction with relevant stakeholders (Ozgen \& Baron, 2007; Strike \& Rerup, 2015) and can for example be observed in symbolic actions (Zott \& Huy, 2007), the use of visual symbols (Clarke, 2011), language (Cornelissen \& Clarke, 2011) and artifacts (Garud \& Guiliani, 2013; Venkataraman et al., 2013).

Entrepreneurial researchers acknowledge the socially situated nature of entrepreneurial cognition (Mitchell et al., 2011). Meanwhile, empirical research into the development of entrepreneurial cognitive structures during opportunity development is sparse (Bingham \& Eisenhardt, 2011). Various authors have called for a closer analysis of micro-level, interactive processes by which business opportunity and entrepreneurial cognition are shaped (Foss et al., 2008; Rasmussen et al., 2011; Shepherd, 2015). This thesis responds to this call by focusing on the micro-level mechanisms on development of entrepreneurial cognition during business opportunity development. The research question central to this thesis is the following:

How do socially situated cognitive mechanisms affect entrepreneurial cognitive development during business opportunity development?

In order to answer this research question, we undertook four empirical studies. Using the grounded theory approach, the sensemaking perspective and discourse analysis, we study entrepreneurs who participated in an institutional incubation/acceleration program. The data stems from weekly logbooks kept by the entrepreneurs during the incubation/acceleration program, as well as from open-ended interviews with those entrepreneurs taken at the moment they exited the program. Based on the results of the empirical studies we identify aspects of socially situated cognitive mechanisms which are instrumental in the co-construction of entrepreneurial sensemaking. Our findings contribute to the conceptualization of the role that socially situated cognitive mechanisms play in entrepreneurial cognitive development during opportunity development.

The rest of this conclusive chapter is built up in the following way. First, we give a summary of each of the four empirical studies contained in this thesis. Next, we draw 
some general, overarching conclusions based on their results. Those results will then be discussed in terms of their theoretical and practical implications. This is followed by an overview of limitations of the empirical research presented in this thesis. Last but not least we make recommendations for future studies regarding socially situated cognitive mechanisms in entrepreneurship.

SUMMARY OF CHAPTER 2: WILD WEST: TARGETED FEEDBACK MECHANISMS AND THEIR EFFECT ON COGNITIVE DEVELOPMENT

In Chapter 2, we explore the effect of two feedback mechanisms - coaching and business panel presentation - on entrepreneurial cognitive development during opportunity development. To do so, we use the grounded theory approach and draw on data from weekly logbooks that the entrepreneurs kept.

Our analysis yields a taxonomy that distinguishes between four categories of entrepreneurs which we label, respectively: 'Greenhorns', 'Cowboys', 'Trappers' and 'Saloon-owners'. These categories with regards to prior entrepreneurial experience and the nature of the value proposition (product vs service). Our results show that both feedback mechanisms contribute to cognitive learning outcomes in terms of the development of declarative, procedural and metacognitive knowledge but that they do so in different ways. Both forms of feedback lead to the development of procedural knowledge. Panel feedback leads to the development declarative knowledge among entrepreneurs which is crucial for business development as well as investor attractiveness (Chen et al, 2009). It also leads to relatively more development of cognitive strategies (metacognitive development), while coaching leads to more metacognitive development in absolute terms. Notably, coaching feedback fosters the development of reflective, meta-cognitive (Haynie et al., 2012; St-Jean \& Audet, 2012) or 'deeper-level' entrepreneurial learning (Krueger, 2007). We find that experienced entrepreneurs report the effect of metacognitive development more often than novice entrepreneurs, while novice entrepreneurs report more development of declarative knowledge.

This study contributes to our understanding of feedback processes by the identification of complimentary effects of coaching and panel feedback mechanisms on the cognitive development of entrepreneurs, as well as the role that prior entrepreneurial experience and the nature of their value proposition play in this. 


\section{Summary of ChAPTER 3: SenSEbreaking AND deVElopment OF ENTREPRENEURIAL COGNITION}

Chapter 3 consists of empirical study on the concept of sensebreaking and how third parties affect entrepreneurial cognitive development by means of sensebreaking. Sensebreaking is analysed in terms of its mechanisms of redirecting, reframing and questioning described by Vlaar et al. (2008).

We find that sensebreaking mechanisms affect the development of entrepreneurial cognition by triggering novel sensemaking of mainly human capital aspects but also, to a lesser extent, sensemaking of social and economic capital. The results show that the mechanisms of redirecting and reframing affect entrepreneurial cognitive development differently. Redirecting, the most frequent reported sensebreaking mechanism, leads to more technical novel sensemaking, such as adaptation of strategy and focus. Meanwhile, reframing stimulates entrepreneurs' reflections on and realization of something completely different and 'out of the box' thinking about the business opportunity. In this way, reframing facilitates the shaping of beliefs and attitudes of entrepreneurs which has been described as deeper level learning (Krueger, 2007).

We also find that not all stakeholder groups contribute to sensebreaking equally. Instead, sense-breaking mechanisms of reframing are more often triggered by stakeholders in the business incubation program than outside that program. Yet, interaction with stakeholders outside the incubation program leads to significantly more questioning occurrences.

The results of this study contribute to a more nuanced theoretical conceptualization of how different sensebreaking mechanisms affect entrepreneurial cognition. Methodologically, we contribute by showing that the novel data collection method of analyzing diary entries employed in this study enables researchers to observe and report on the origins of entrepreneurial cognition with very limited retrospection bias. Our study yields insights into the functions of institutional support program - particularly, in providing a demonstration of the influence of different program staff - mentors or trainers - on reframing and redirecting mechanisms triggered by intersubjective sensebreaking. Particularly, we found that reframing can prove valuable in creating 'cognitive space' for novel assessments and subsequent decision-making about the value of a resource. Meanwhile, redirecting facilitates incremental learning episodes, such as shifting the entrepreneur's attention to a different market or a novel partner in value creation. Practically, the results of our study are also useful in clarifying our existing understanding of role of critical feedback provided inside business incubation programs. 


\section{Summary of Chapter 4: Mental MOdels of MARKet ORIENTATION}

In Chapter 4 we explore the cognitive dimension of developing a market orientation. Central to this research are the key concepts of a market orientation and the mental model. A market orientation is defined as an orientation towards the creation of (superior) customer value. The existing measures of market orientation lack applicability in the case of smaller businesses (Raju et al, 2011), which includes start-up ventures or self-employed entrepreneurs. Taking a grounded theory approach, we examine sensemaking processes of entrepreneurs who are engaged in solving problems encountered in the creation of (superior) customer value during business opportunity development.

The results of our analysis yield six market-oriented mental models which we labelled 'Customer orientation', 'Communication', 'Leverage of Networks', 'Collaboration', 'Team' and 'Proactivity'. While we find that the mental model 'Team' links to what has been called 'inter-functional coordination' (Narver \& Slater, 1990) or information dissemination, we do not find mental models which reflect a development of the so-called 'competitor orientation' (Narver \& Slater, 1990) which is in line with the existing literature on a market orientation in nascent ventures (Roersen et al., 2013). In addition, our results show that market-oriented mental models used by experienced entrepreneurs are more general and tacit in nature.

Our findings contribute to the conceptualization of the role and significance of stakeholders in solving problems related to creating (superior) customer value and point towards the development of a stakeholder orientation among entrepreneurs as part of their market orientation. Existing market orientation literature emphasizes the importance of (potential) customer engagement, either in terms of an orientation (Narver \& Slater, 1990 ) or in terms of information generation and dissemination to customers (Kohli \& Jaworski, 1990). But the results of this study yield empirical insights into the predominance of a stakeholder dimension of the market orientation, an aspect that has been undertheorized in existing market orientation literature. These findings underline the role of the social context in entrepreneurial sensemaking which is characteristic for the socially situated cognitive conceptualization of entrepreneurial cognition (Mitchell et al., 2011).

We also show how market-oriented mental models used by entrepreneurs with prior entrepreneurial experience reflect a more expertly skilled performance as described 
by Dreyfus \& Dreyfus (2005) which affects the way that experienced entrepreneurs could communicate their market-oriented problem to other members of the venture in the case of venture growth and the ensuing development of a venture-level market orientation.

Our results contribute to theory-building of how a market orientation develops among individuals, called for in the literature (Morgan et al., 2009), by a conceptualization of micro-level roots of the venture-level phenomenon of a market orientation. More practically, insights from this study can help entrepreneurs to understand the importance of stakeholders for problem solving during the creation of (superior) customer value. This study can also help to optimize the design of business support programs. For example, those programs could benefit from a focus not only creating customer value, but from the identification of relevant stakeholders for an optimal 'community of inquiry' (Shepherd \& Patzelt, 2017) in which entrepreneurs receive feedback on their business idea. Also, we found that nascent as well as experienced entrepreneurs do not report mental models that reflect a competitor orientation, while the literature on market orientation suggests that an orientation towards competitors contributes to a firm's profitability (Narver \& Slater, 1990). Incubation and acceleration support structures can, by means of trainings, make entrepreneurs more conscious of developing all dimensions of a marketorientation. This facilitates the development of the business opportunity into a commercially viable market offer, with a positive effect on the venture's performance in terms of profitability.

\section{SUMmary OF CHAPTER 5: SENSEMAKIng OF THE BUSINESS MODEL CONCEPT}

In Chapter 5, we explore entrepreneurial sensemaking of the business model artifact. The business model is seen as the organizational and financial 'architecture' of a business (Teece, 2010). Osterwalder et al. (2005) describe three categories into which literature on business models can be classified. Those categories are increasingly abstract. In the category of highest abstraction level, the business model artifact is an abstract representation of a value creation logic.

Approached in its most abstract capacity of a model, the business model artifact can fulfill different purposes. Based on a review of existing literature we distinguish between four distinctive purposes for which the business model artifact can be used. We then looked at entrepreneurial sensemaking processes of the business model artifact for these different purposes. In particular, we were interested in analyzing whether there are differences in entrepreneurial sensemaking of those purposes based on their 
educational background. Research has shown that entrepreneurs with a background in STEM disciplines (science, technology, engineering and mathematics) often have a tendency to focus only on the technical aspects of innovation which makes successful commercialisation more difficult (Berry, 1996).

The results of our study show that entrepreneurs make sense of the business model artifact mainly for purposes of understanding and developing the venture's value creation logic. To a lesser extent, entrepreneurs also use the business model artifact to communicate about and analyze the venture's value creation logic. In addition, our findings show that entrepreneurs with a STEM education are twice as likely to use the communication purpose; they report using this purpose for both external as well as internal interactions, for example to share and discuss the value creation logic with team members, while non-STEM entrepreneurs solely report the use of the business model artifact for communicative purpose to external parties. Used for its communicative purpose, the business model artifact provides entrepreneurs with a mechanism to involve relevant stakeholders in the creation of shared meaning, crucial for the successful creation of value (Clarke \& Cornelissen, 2014).

Our study contributes to understanding how the business model artifact provides a mechanism for entrepreneurs to move from a 'technical mindset' (Berry, 1996) to a more 'stakeholder value-oriented' mindset by using that artifact for its communicative purpose. On a practical level, we suggest that the business model artifact can be leveraged by practitioners during trainings in venture incubation programs. Trainings in the use of the different purposes for which the business model artifact can be used also enhance the use of the analytical and communicative purposes of the venture's business model.

\subsection{Overall Conclusion}

The four empirical studies contained in this thesis reveal the ways in which entrepreneurial cognition develops by means of different socially situated cognitive mechanisms. Those socially situated cognitive mechanisms are respectively coaching and panel feedback, sensebreaking mechanisms, stakeholder-oriented mental models and the business model artifact. 
In Chapter 2 we showed that both coaching and panel feedback lead to learning of procedural knowledge (or knowledge organization) among entrepreneurs. In addition, coaching feedback leads to the development of cognitive strategies (or meta-cognitive knowledge) more often than panel feedback, while panel feedback more often leads to learning of declarative knowledge (verbal knowledge) than coaching feedback. In Chapter 3, we saw how certain stakeholders trigger the development of novel sensemaking by means of different sensebreaking mechanisms, and that some lead to metacognitive development of entrepreneurs. In Chapter 4 we analyzed the development of a market orientation among entrepreneurs and found that stakeholderoriented mental models function as a mechanism for the engagement with stakeholders in problem solving during opportunity development. And we see in the results of Chapter 5 that the communicating purpose of the business model artifact functions as a mechanism to involve relevant stakeholders in opportunity development.

The different mechanisms we examine in this thesis - feedback mechanisms, sensebreaking mechanisms, market-oriented mental models and the business model artifact - are situated in a specific context that contains various stakeholders who form an entrepreneur's 'community of inquiry' (Shepherd \& Patzelt, 2017) which provide relevant feedback to the entrepreneurs, thus shaping entrepreneurial sensemaking and ultimately the entrepreneur's business opportunity. In that sense, those mechanisms are socially situated, cognitive mechanisms by which third parties can shape entrepreneurial cognition.

Business opportunity development requires the making of shared meaning about what an opportunity's (future) value is or could be in the market realm. During opportunity identification and preparation, entrepreneurs seek to create real customer value. That customer value is 'in making' and is not yet a reality and therefore must be interactively legitimized, by socially constructing meaning through the use of narratives, signs or symbols (Gardu \& Guiliani, 2013; Zott \& Huy, 2007; Downing, 2005).

Entrepreneurs can use socially situated cognitive mechanisms such as marketoriented mental models and the business model artifact to engage stakeholders in the co-construction of entrepreneurial sensemaking. These mechanisms are relevant for entrepreneurs who must move from a product orientation to a market orientation in order to create a commercially successful business opportunity; and as Roersen et al. (2013) point out this does not necessarily happen. The results of the study presented in Chapter 4 show that a market orientation is not only about customers but even more so about 
relevant stakeholders. As we see in Chapter 5, the business model artifact can be used effectively to engage stakeholders in the entrepreneur's 'community of inquiry' (Shepherd \& Patzelt, 2017) in developing the venture's business model and in learning how to engage those stakeholders - by using the business model artifact for its communicative purposes.

On the basis of our results we conclude that socially situated cognitive mechanisms function as enabling and facilitating instruments for the co-construction of entrepreneurial sensemaking and ultimately for the development of entrepreneurial cognition during opportunity development. We saw that not only stakeholders use socially situated cognitive mechanisms - such as targeted feedback mechanism and more concretely, sensebreaking mechanisms - to affect the development of entrepreneurial cognition by means of co-constructed novel sensemaking. Also, socially situated cognitive mechanisms used by entrepreneurs - such as market-oriented mental models and the business model artifact - drive the co-construction of meaning. These socially situated cognitive mechanisms help to develop the entrepreneurs' awareness for or facilitating the engagement in interaction with relevant stakeholders during opportunity development. This not only affects the entrepreneur's own cognitive development, but ultimately the construction of shared meaning with stakeholders in terms of a legitimate business opportunity.

\subsection{THEORETICAL IMPLICATIONS AND CONTRIBUTIONS}

\section{THE CONTRIBUTION OF THE SENSEMAKING PERSPECTIVE}

The empirical studies of this thesis demonstrate that the sensemaking perspective can be used either with grounded theory method or combined with existing theory in order to 'dig deeper' into the variables that shape entrepreneurial cognition and help understand novel sensemaking processes, cognitive development more generally and ultimately how and why entrepreneurs engage in certain actions and decision-making processes which affect venture development.

We identify three ways in which the sensemaking perspective contributes to studying socially situated cognitive phenomena. For one, we are able to take into account the role of the social context and (im)material artifacts in the development of entrepreneurial cognition which contributes to our understanding of co-construction of 
entrepreneurial sensemaking. Second, using the sensemaking perspective for the study of socially situated cognitive phenomena yields insights into mechanisms that enable successful sensegiving as defined by Gioia \& Chittipetti (1991). Third, the sensemaking perspective facilitates theory-building on the concept of metacognitive or 'deeper level' cognitive learning described by Krueger (2003; 2007). All three contributions will be discussed individually below.

First, the sensemaking perspective helps to analyze and understand the perception of entrepreneurs, their perspective and impressions associated with the coconstruction of shared meaning. This is particularly relevant for the identification of how the social context affect the entrepreneurs' meaning-making activities. The sensemaking perspective's focus on identity, legitimacy and their temporal dimensions are also central to narrative perspectives in entrepreneurship (Downing, 2005; Fletcher, 2007; Gartner, 2007) which emphasize the role of language and communication in business opportunity development. In addition to the analysis of narratives, the sensemaking perspective also facilitates the analysis of the use of artifacts in entrepreneurship which is recognized as important (Venkataraman et al., 2013). Therefore, the sensemaking perspective helps to examine how interpretative processes by entrepreneurs are shaped and co-constructed through interaction with other parties and through the use of (im)material artifacts during the interaction with those stakeholders.

Second, this thesis yields valuable insights into the way the sensemaking perspective is useful for the study of sensegiving. Weick (2012) sees sensemaking as episodic but contextualized as it involves the connection of a cue to a frame: "A concrete individual entity is categorized into an idealized general one and becomes meaningful. A cue, by itself, without a frame, has no predicate. Once you put it in a frame, it does." (Weick, 2012: p. 148). While the literature has made a distinction between sensegiving and sensemaking in which sensegiving is defined as 'the indended' and sensemaking as 'the perceived' (Gioia \& Chittipeddi, 1991), sensegiving is an essential part of shared sensemaking because it presupposes the existence of a frame ('the intended') to which a certain cue ('the perceived') can be linked - thereby fulfilling the presupposition of a certain intent. For example, the study presented in Chapter 4, on market-oriented mental models, shows how entrepreneurs are increasingly intentional in using stakeholders for the solution of problems related to the creation of customer value.

Third, the sensemaking perspective is useful for the analysis of metacognitive development of entrepreneurs. Metacognition is related to deep belief structures which 
form and influence the entrepreneurial mind (Krueger, 2007; Mitchell et al., 2007). Those deep belief structures are how we represent and process knowledge and information and are also involved in meta-cognitive knowledge. Metacognitive processes can exert control over automatic behavior and underlie the ability to explicitly share experiences with other agents, such as in reflective discussion (Frith \& Frith, 2012). Baron (2014) defined the development of accurate metacognition as one of the intriguing questions meriting more attention by entrepreneurship scholars. The study contained in Chapter 3 on sensebreaking mechanisms for example reveal the role that reframing plays in triggering metacognitive learning among entrepreneurs and how their novel sensemaking reflects a different meaning as a result of those reframing occurrences.

\section{HOW SOCIALLY SITUATED COGNITIVE MECHANISMS ENHANCE LEARNING ABOUT LANGUAGE}

Market participants do not share the same information or have the same understanding of events and situations - for example, they employ other conceptual categories, or interpret information differently (Langlois, 2007). Therefore the role of language is a key factor in the co-construction of sense. Clarke \& Cornelissen (2014) posit that language shapes entrepreneurial cognition by providing a means to verbalize one's ideas and "shape or limit the ways in which the speaker forms conceptions of the world" (Clarke \& Cornelissen, 2014: p. 387) and that "it is important to recognize the formative role of language in conceptualizing venture opportunities and in influencing stakeholders about the feasibility of a venture." (Clarke \& Cornelissen, 2011: p.776). The authors underline that language exemplifies the socially situated nature of cognition because it is primarily via language that actors encode and articulate novel ideas and make them recognizable to others, which contributes to sharing them with relevant stakeholders - for example, to receive valuable feedback during opportunity development.

The results of our research show that language enables (meta)cognitive development of entrepreneurs by means of socially situated cognitive mechanisms. Both targeted feedback mechanisms as well as the sensebreaking mechanisms studied in Chapter 2 respectively Chapter 3 consist of verbal feedback through which relevant concepts and ideas for opportunity development are transferred to entrepreneurs. Also, the role of verbal exchange is reflected in the empirical study presented in Chapter 4, such as the entrepreneurs' development of the mental model of 'Communication'. And in the study presented in Chapter 5 we saw how the business model artifact is used intentionally for communicating purposes in the interaction with stakeholders, in order to 
discuss and make sense of the venture's value creation logic together with, for example, team members or financial investors.

The empirical studies in this dissertation demonstrate how entrepreneurs develop (meta-) cognitive skills through communicative interaction. In other words: Entrepreneurs learn about learning via language, by talking with stakeholders who provide relevant concepts and ideas on how to shape the business opportunity. These interactive processes shape the way in which the entrepreneurs think and talk about their business opportunity or value creation logic, and also shape how they learns about developing that business opportunity.

Socially situated cognitive mechanisms enable the encoding and articulating of novel ideas by providing a tool for stakeholders to engage with entrepreneurs, respectively for entrepreneurs to engage with stakeholders. These tools are used by both sides to provide stakeholders with opportunities to shape and influence the entrepreneur's cognition by providing him with novel information, concepts, ideas or perspectives. This in turn affects the development of the opportunity itself. This thesis thus contributes to the conceptualization of the role of socially situated cognitive mechanisms in entrepreneurship, by illustrating the ways which language 'works' through them, and how they facilitate not only entrepreneurial sensemaking but ultimately the development of the business opportunity.

\section{METHODICAL IMPLICATIONS: DIARY RESEARCH}

The diary method used in two empirical studies of this thesis contributes to our understanding of the process and content on diary research in entrepreneurship studies. By employing this method, we answer to Shepherd's (2015) call for methodological experimentation and modernisation in scientific entrepreneurship research. In using the diary method in studying entrepreneurial sensemaking, researchers can avoid commonly encountered difficulties with the reliability and validity of the data, such as avoiding socially desirable or retrofitted answers by entrepreneurs under investigation.

The diary method enables the observation of real-time, individual-level interactions between entrepreneurs and specific stakeholders over time, as well as their effects on the cognitive development of entrepreneurs. It also facilitates the observation of entrepreneurial cognitive processes - in terms of their impressions and interpretations of events - with very limited retrospection bias. We therefore suggest that using this 
method helps to avoid methodological pitfalls in qualitative research on the phenomenon of entrepreneurship. While applications of diary methods are scarce in the entrepreneurial literature with the exception of Kato and Wiklund (2011) who analyzed blog entries of entrepreneurs, this method contributes to expanding the array of research methods in entrepreneurship, in particularly on the micro-level interactions which affect opportunity development.

\subsection{PRACTICAL IMPLications AND CONTRIBUtions}

The results of the empirical studies shed light on the role of incubation/acceleration program such as the VLT program which provided the research setting. Below we discuss our findings for entrepreneurs, as well as for the design of entrepreneurship education and for policy-making regarding entrepreneurship support structures.

\section{IMPLICATIONS FOR ENTREPRENEURS}

Entrepreneurs can leverage the insights presented in the results of the empirical studies to reflect on and become (more) aware of being a particular 'type' of entrepreneur, particularly related to their previous entrepreneurial experience and the type of opportunity they are developing. As we saw in Chapter 2, coaching and panel presentation feedback affect cognitive learning outcomes differently depending on whether entrepreneurs are novice or experienced and whether they develop a product- or service-based value offer. Becoming aware of one's own needs - and that one size does not fit all - is an important step towards articulating those needs and, accordingly, leveraging the complementary effects of coaching and panel feedback. In particular, novice entrepreneurs can use cognitive development in terms of declarative knowledge provided by panel members to create focus in which market they are targeting and find out who is relevant for their 'community of inquiry' (Shepherd \& Patzelt, 2017) which plays an important role in providing further feedback and shaping of the business opportunity.

Generally, as shown in Chapters 3, 4 and 5, entrepreneurs can use socially situated cognitive mechanisms to leverage feedback provided by stakeholders, which in turn leads to cognitive development. This occurs for example by creating developing a stakeholder orientation in terms of stakeholder-oriented mental models and the use of the 
business model artifact for the purpose of communicating with relevant stakeholders. This mechanisms lead to the engagement with stakeholders who provide feedback, new information and knowledge to the entrepreneur and who thereby stimulate the entrepreneur's cognitive development.

In sum, awareness of how an entrepreneur's background and type of value offering can help them to become more skilled in seeking the right kind of feedback and assistance. This in turn contributes to the way they can leverage socially situated cognitive mechanisms more effectively, in order to optimize their (cognitive) learning experiences during opportunity development.

\section{IMPLICATIONS FOR ENTREPRENEURSHIP EDUCATION}

Corbett (2005) proposed that students in entrepreneurship courses should learn more about how to adapt their original ideas in reaction to changes suggested by potential customers and other actors in the market place. We propose that socially situated cognitive mechanisms analyzed in this thesis are enabling tools for adaptation and change of business opportunities, insofar as that they offer platforms for interactive engagement with other parties and expose individuals to new information, ideas and perspectives.

As we saw in Chapters 2 and 3, stakeholder feedback in the form of targeted feedback and sensebreaking mechanisms is valuable for (meta)cognitive development of entrepreneurs. By raising awareness among entrepreneurship students for the different mechanisms of targeted feedback and sensebreaking in combination with using the business model artifact - such as the Osterwalder \& Pigneur's (2010) business model canvas - those socially situated cognitive mechanisms can become useful tools for stimulating the development of entrepreneurial (meta)cognitive skills and facilitate subsequent changes in thinking patterns, more flexibility in perspective taking and ultimately facilitate the students' openness and willingness to adapt a business idea.

While feedback-based learning is relevant in entrepreneurship education in higher education, it is also relevant for academics' engagement in value creation networks, such as multidisciplinary research teams or public-private teams designed to tackle interdisciplinary research topics, such as public safety or humanoid robotics. The socially situated cognitive mechanisms analyzed in this thesis are suitable for stimulating and sustaining effective feedback-based (entrepreneurship) education because they can easily be integrated into experiential learning approaches. Those approaches emphasize the development of soft skills, such as presentational skills and interpersonal 
communication, which are considered important for higher education in general (Kolb \& Kolb, 2005) and in entrepreneurial thinking in particular (Krueger, 2007). The training in and subsequent conscious leverage of these mechanisms by individuals or in teams could be particularly valuable for multidisciplinary trajectories, not only in entrepreneurship education but also in multidisciplinary research projects.

\section{ENTREPRENEURSHIP SUPPORT STRUCTURES}

This thesis provides empirical underpinnings for the role of stakeholders in the development of entrepreneurial cognition. These insights can be used to optimize the design of business incubation/acceleration programs. In particular, our research findings show that socially situated cognitive mechanisms represent enabling tools for actors in business support settings to 'steer', focus and target practice-based entrepreneurial learning. For example they yield evidence that entrepreneurial cognitive development can be achieved by targeted feedback mechanisms and by sensebreaking mechanisms examined in Chapters 2 and 3.

We saw in the first empirical studies that the two targeted feedback mechanisms of coaching and panel feedback affect different categories of entrepreneurs in different ways, and even have complementary effects on entrepreneurs. By combining those feedback mechanisms, in particular for novice entrepreneurs with a service-offer as well as for experienced entrepreneurs who seek to transition from a service-offer to a product offer, the combination of coaching interaction with panel presentations can yield effective cognitive learning outcomes in the realm of knowledge organization and cognitive strategies. In that way these studies provide empirical, micro-level evidence for the role that coaching and panel presentation play in the entrepreneurial (meta)cognitive development.

In addition, trainings in using the business model artifact for different purposes provide entrepreneurs with an instrument to not only formulate and define but also analyze and communicate their opportunity's value creation logic. Offering those targeted feedback mechanisms as well as trainings on the business model artifact will enhance the entrepreneurs' cognitive development, the development of market-oriented metal models and positively affect stakeholder engagement. Raising of awareness for a stakeholder orientation as well as for the business model artifact by means of trainings can help to facilitate the set-up of a 'community of inquiry' (Shepherd \& Patzelt, 2017) in 
which entrepreneurs can engage in thought exchange and leverage feedback from relevant stakeholders that help to shape the business opportunity.

The socially situated cognitive mechanisms we identified - coaching and panel feedback and the use of the business model artifact - can also be used for monitoring and evaluation of support programs. Policy-makers can leverage the conceptualization of the process and outcomes of stakeholder interaction offered in the empirical studies, in order to develop indicators for monitoring and evaluation of the efficient use of both coaching and panel interactions in support programs.

\subsection{RESEARCH LIMITATIONS}

In this section, the limitations of the empirical studies that we identified are discussed.

Firstly, our research population consists of a relatively small number of entrepreneurs, a constraint often encountered in qualitative research. This means that they do not always yield quantifiably measurable causal correlations which can explain or predict the functioning of the socially situated cognitive mechanisms we analyzed. Due to the limited sample size we were unable to analyze the effects of other variables which could affect entrepreneurial cognitive development, such as differences in gender, educational background or team composition. Authors have found that these factors matter in opportunity development (Mueller \& Thomas, 2001); yet due to time and resource restraints posed by the composition of a thesis those factors could not be taken into account in this study. However, this thesis contributes to the conceptualization of entrepreneurial cognitive development by identifying how socially situated cognitive mechanisms are relevant for the cognitive development of entrepreneurs. By doing so, this thesis provides 'legwork' by laying a basis for the design of further, explanatory studies in the area of entrepreneurial cognitive development.

A second limitation concerns the form of the data, specifically the diary data which is a very 'raw' form of data; the entrepreneurs were free to write down thoughts about their development, only guided by the four 'topics' within each weekly diary, and subsequently entrepreneurs varied in terms of content and frequency of self-reporting. 
We did notice that some entrepreneurs enjoyed the exercise, while others at times lacked the time, commitment and discipline required for consistent self-reporting given the fact that their new venture kept them very busy. This appeared to affect the level of detail and elaboration with which the diaries were kept. Also, some entrepreneurs seemed cautious about which information to report in the diaries, for reasons of confidentiality and secrecy. In order to provide a maximum level of comfort to the entrepreneurs, all information given in the diaries were maintained in secure servers with very limited access to the employees at the business accelerator.

A third limitation of this study is associated with the research setting. Our data stems from a sample of entrepreneurs who are part of a research incubation/acceleration program. This means that our sample is self-selectively biased, consisting of entrepreneurs who chose to actively leverage institutionally-set resources for the development of their business opportunity. Because the studies are undertaken in an institutional support context our findings are not necessarily generalizable to all entrepreneurs. We cannot compare entrepreneurs inside an institutional incubation/acceleration support environment with those who 'independently' pursue the realization of their business opportunity outside the resource-rich environment of the incubation/acceleration program. Therefore we cannot exclude the possibility of a biased research population characteristically unwilling or unable to independently pursue business development but clever enough to seek help. However, our sample consists of a diverse selection of educational and industrial backgrounds. In that way we sought to validate the results across a wide variety of entrepreneurs and increase the reliability of our findings.

\subsection{FUTURE RESEARCH}

In terms of avenues for future research, we discern the following topics worth investigating.

\section{SELF-REPORTS: A FUTURE ALLEY OF DATA COLLECTION IN ENTREPRENEURSHIP STUDIES}

While qualitative data is increasingly seen as important in business research (Watson, 2011), there is still a world to discover in terms of applying qualitative research methods - such as the sensemaking perspective - to the study of entrepreneurship. For example we know that feedback has a positive effect on opportunity recognition (Ozgen 
\& Baron, 2007) and cognitive learning (St-Jean \& Audet, 2012), future studies on the qualitative nature of said feedback, and its effects, can advance more detailed insights into this aspect of entrepreneurial cognitive development. In particular, longitudinal studies on how socially situated cognitive mechanisms affect affect entrepreneurial cognition could yield more insights into the effects of third party involvement during business opportunity development.

More fine-grained data collection methods and analyses can provide understanding of unique entrepreneurial processes, whether cognitive or action-based. Modern technology and multimedia offer proficient means for academics to design novel research instruments, for example in the form of logbooks, short surveys, or social media applications. We therefore agree with Foo, Uy \& Baron (2009) who advocate a more extensive use and critical evaluation of these methods.

StUdying the EXPLANATORY ROLE OF SOCIALly SITUATED COGNITIVE MECHANISMS IN ENTREPRENEURIAL COGNITIVE DEVELOPMENT

Theory-building on the role of socially situated cognition in entrepreneurship is seen as a promising research avenue (Mitchell et al., 2011; Clarke \& Cornelissen, 2014). In particular, the role of socially situated cognitive mechanisms in the development of metacognitive abilities can advance our understanding of the development of different metacognitive abilities. In particular, as we saw in Chapter 3, the sensebreaking mechanism of reframing leads to metacognitive development or 'deeper level learning' (Krueger, 2007) of entrepreneurs, by means of triggering novel sensemaking processes among entrepreneurs related to the adoption of a different perspective on how to develop their business or the critical reflection on their entrepreneurial identity, their values or motivations. Future empirical examination of sensebreaking effects could use the concept of reframing as an analytical tool to examine which stakeholders trigger reframing and how this affects 'deeper level learning', and ultimately the development of an entrepreneurial mindset. For example, Haynie et al. (2010) distinguish between two forms of metacognitive resources; metacognitive knowledge and metacognitive experience. Research into the effect of stakeholder feedback on the development of these metacognitive resources could yield valuable information about how those resources are developed by means of different targeted feedback mechanisms.

Future studies on socially situated cognitive mechanisms should also focus on theory-building of explanatory factors for the functioning of these mechanisms. We saw 
in Chapter 2 that coaching and panel feedback have different effects on entrepreneurs, depending on entrepreneurial attributes such as prior entrepreneurial experience and the nature of the business opportunity which is developed. The examination of how personal attributes - such as prior professional experience, age or gender - influence the use and effect of socially situated cognitive mechanisms can add to our conceptualization of socially situated cognition in entrepreneurship. On a related note, future studies on the relationship between socially situated cognitive mechanisms and venture survival, growth and employment generation would yield valuable insights into the role that co-constructed sensemaking plays in the commercial success of entrepreneurial ventures.

\section{THE ROLE OF AFFECT IN SOCIALLY SITUATED COGNITIVE MECHANISMS}

Shepherd (2015) emphasizes the role of emotions in cognitive development of entrepreneurs, and the relevance of research on this topic. Particularly interesting in this context is the role of passion in enabling socially situated cognitive mechanisms. Cardon et al. (2009) argue that passion is a critical element in an entrepreneur's identification with his role and tasks as entrepreneurs because it enhances commitment and keeps the energy focused on achieving his goals. Also, the role of distrust and anger or frustration could be powerful mediators and therefore worth investigating in future studies on the role of socially situated cognitive mechanisms.

On a related note, another factor related to emotions and worth exploring is that of trust. In general, trust is relevant for the integration of weak and strong tie networks (Scarborough et al., 2013) and could affect the access to and frequency of socially situated cognitive mechanisms. For example, Bammens \& Collewaert (2014) found that trust plays a role in entrepreneur-angel investor relationships, both positively and negatively. In chapter 3 we saw that financial investors are a relevant party for exposing entrepreneurs to sensebreaking instances. These results showed that investors affect novel entrepreneurial sensemaking, most notably in terms of questioning occurrences. It might be a fruitful endeavor to study the level of trust between certain stakeholders and entrepreneurs who are relevant for sensebreaking, or examine the effect of different forms of trust type - such as described by Scarbrough et al. (2013) - on the occurrences and effects of the different sensebreaking mechanisms. 


\subsection{LAST WORDS: THE VALUE OF ANALYZING SOCIALLY SITUATED COGNITIVE MECHANISMS}

This thesis shows that the concept of socially situated cognitive mechanism offers a useful micro-analytical tool for studying the co-construction of entrepreneurial sensemaking. There is merit in examining those kinds of micro-level processes for the following reason. As this thesis shows, those stakeholders play a central role in the coconstruction of entrepreneurial sensemaking and ultimately in the formation of an entrepreneurial mindset. That mindset is becoming increasingly important for the network economy of the $21^{\text {st }}$ century in which soft skills, pro-activeness and a stakeholder orientation are crucial for value capture, creation and delivery.

The advent of the modern-day network economy necessitates the development of entrepreneurial cognition in order to engage in and leverage business opportunities together with stakeholders. We believe that this dissertation provides valuable insights into the instrumental role of socially situated cognitive mechanisms in the development of entrepreneurial cognition and, more generally, an entrepreneurial mindset.

\subsection{BIBLIOGRAPHY}

Ardichvili, A., Cardozo, R., \& Ray, S. (2003). A theory of entrepreneurial opportunity identification and development. Journal of Business venturing, 18(1), 105-123.

Bammens, Y., \& Collewaert, V. (2012). Trust between entrepreneurs and angel investors exploring positive and negative implications for venture performance assessments. Journal of Management, 0149206312463937.

Baron, R. A., \& Ensley, M. D. (2006). Opportunity recognition as the detection of meaningful patterns: Evidence from comparisons of novice and experienced entrepreneurs. Management science, 52(9), 1331-1344.

Baron, R. A. (2009). Effectual versus predictive logics in entrepreneurial decision making: Differences between experts and novices: Does experience in starting new 
ventures change the way entrepreneurs think? Perhaps, but for now,"caution" is essential. Journal of Business Venturing, 24(4), 310-315.

Baron, R. A. (2014). Essentials of entrepreneurship: evidence and practice. Edward Elgar Publishing.

Berry, M. M. (1996). Technical entrepreneurship, strategic awareness and corporate transformation in small high-tech firms. Technovation, 16(9), 487-522.

Bingham, C. B., \& Eisenhardt, K. M. (2011). Rational heuristics: the 'simple rules' that strategists learn from process experience. Strategic Management Journal, 32(13), 14371464.

Cardon, M. S., Wincent, J., Singh, J., \& Drnovsek, M. (2009). The nature and experience of entrepreneurial passion. Academy of management Review, 34(3), 511-532.

Chen, X.-P., Yao, X., and Kotha, S. Entrepreneur Passion And Preparedness In Business Plan Presentations: A Persuasion Analysis Of Venture Capitalists' Funding Decisions. Academy of Management Journal 52, 1 (2009), 199-214.

Chesbrough, H. (2010). Business model innovation: opportunities and barriers. Long range planning, 43(2), 354-363.

Clarke, J. S., \& Cornelissen, J. P. (2014). 11. How language shapes thought: new vistas for entrepreneurship research. Handbook of Entrepreneurial Cognition, 383.

Corbett, A. C. (2005). Experiential learning within the process of opportunity identification and exploitation. Entrepreneurship Theory and Practice, 29(4), 473-491.

Clarke, J. (2011). Revitalizing entrepreneurship: how visual symbols are used in entrepreneurial performances. Journal of Management Studies, 48(6), 1365-1391.

Clarke, J., \& Cornelissen, J. (2011). Language, communication, and socially situated cognition in entrepreneurship. Academy of Management Review, 36(4), 776-778.

Clarke, J. S., \& Cornelissen, J. P. (2014). 11. How language shapes thought: new vistas for entrepreneurship research. Handbook of Entrepreneurial Cognition, 383. 
Cull, J. (2006). Mentoring young entrepreneurs: what leads to success?. International journal of evidence based coaching and mentoring, 4(2), 8-18.

Dew, N., Read, S., Sarasvathy, S. D., \& Wiltbank, R. (2015). Entrepreneurial expertize and the use of control. Journal of Business Venturing Insights, 4, 30-37.

Downing, S. (2005). The social construction of entrepreneurship: Narrative and dramatic processes in the coproduction of organizations and identities. Entrepreneurship theory and Practice, 29(2), 185-204.

Fletcher, D. (2007). 'Toy Story': The narrative world of entrepreneurship and the creation of interpretive communities. Journal of Business Venturing, 22(5), 649-672.

Foo, M. D., Uy, M. A., \& Baron, R. A. (2009). How do feelings influence effort? An empirical study of entrepreneurs' affect and venture effort. Journal of Applied Psychology, 94(4), 1086.

Foss, N. J., Klein, P. G., Kor, Y. Y., \& Mahoney, J. T. (2008). Entrepreneurship, subjectivism, and the resource-based view: toward a new synthesis. Strategic Entrepreneurship Journal, 2(1), 73-94.

Frith, C. D., \& Frith, U. (2012). Mechanisms of social cognition. Annual review of psychology, 63, 287-313.

Gartner, W. B. (2007). Entrepreneurial narrative and a science of the imagination. Journal of Business Venturing, 22(5), 613-627.

Garud, R., \& Giuliani, A. P. (2013). A narrative perspective on entrepreneurial opportunities. Academy of Management Review, 38(1), 157-160.

Gioia, D. A., Thomas, J. B., Clark, S. M., \& Chittipeddi, K. (1994). Symbolism and strategic change in academia: The dynamics of sensemaking and influence. Organization science, 5(3), 363-383. 
Haynie, J. M., Shepherd, D., Mosakowski, E., \& Earley, P. C. (2010). A situated metacognitive model of the entrepreneurial mindset. Journal of business venturing, 25(2), 217-229.

Haynie, J. M., Shepherd, D. A., \& Patzelt, H. (2012). Cognitive adaptability and an entrepreneurial task: The role of metacognitive ability and feedback. Entrepreneurship Theory and Practice, 36(2), 237-265.

Kato, S., \& Wiklund, J. (2011). Doing Good To Feel Good-A Theory Of Entrepreneurial Action Based In Hedonic Psychology. Frontiers of Entrepreneurship Research, 31(4), 1.

Knight, F. H. (1921). Risk, uncertainty and profit. New York: Hart, Schaffner and Marx.

Kolb, A. Y., \& Kolb, D. A. (2005). Learning styles and learning spaces: Enhancing experiential learning in higher education. Academy of management learning \& education, 4(2), 193-212.

Krueger Jr, N. F. (2003). The cognitive psychology of entrepreneurship. In Handbook of entrepreneurship research (pp. 105-140). Springer US.

Krueger, N. F. (2007). What lies beneath? The experiential essence of entrepreneurial thinking. Entrepreneurship theory and practice, 31(1), 123-138.

Langlois, R. N. (2007). The entrepreneurial theory of the firm and the theory of the entrepreneurial firm. Journal of Management Studies, 44(7), 1107-1124.

McMullen, J. S., \& Dimov, D. (2013). Time and the entrepreneurial journey: The problems and promise of studying entrepreneurship as a process. Journal of Management Studies, 50(8), 1481-1512.

Mitchell, R. K., Busenitz, L., Lant, T., McDougall, P. P., Morse, E. A., \& Smith, J. B. (2002). Toward a theory of entrepreneurial cognition: Rethinking the people side of entrepreneurship research. Entrepreneurship theory and practice, 27(2), 93-104. 
Mitchell, R. K., Busenitz, L. W., Bird, B., Marie Gaglio, C., McMullen, J. S., Morse, E. A., \& Smith, J. B. (2007). The central question in entrepreneurial cognition research 2007. Entrepreneurship theory and practice, 31(1), 1-27.

Mitchell, R. K., Randolph-Seng, B., \& Mitchell, J. R. (2011). Socially situated cognition: Imagining new opportunities for entrepreneurship research. Academy of Management Review, 36(4), 774-776.

Morgan, N. A., Vorhies, D. W., \& Mason, C. H. (2009). Market orientation, marketing capabilities, and firm performance. Strategic Management Journal, 30(8), 909-920.

Narver, J. C., \& Slater, S. F. (1990). The effect of a market orientation on business profitability. The Journal of marketing, 20-35.

Osterwalder, A., Pigneur, Y., \& Tucci, C. L. (2005). Clarifying business models: Origins, present, and future of the concept. Communications of the association for Information Systems, 16(1), 1.

Osterwalder, A., \& Pigneur, Y. (2010). Business model generation: a handbook for visionaries, game changers, and challengers. John Wiley \& Sons.

Ozgen, E., \& Baron, R. A. (2007). Social sources of information in opportunity recognition: Effects of mentors, industry networks, and professional forums.Journal of business venturing, 22(2), 174-192.

Radu Lefebvre, M., \& Redien-Collot, R. (2013). How to do things with words: The discursive dimension of experiential learning in entrepreneurial mentoring dyads. Journal of Small Business Management, 51(3), 370-393.

Rasmussen, E., Mosey, S., \& Wright, M. (2011). The evolution of entrepreneurial competencies: A longitudinal study of university spin-off venture emergence. Journal of Management Studies, 48(6), 1314-1345. 
Roersen, M. J., Kraaijenbrink, J., \& Groen, A. J. (2013). Marketing Ignorance and the Validity of Narver and Slater's MKTOR Scale in High-Tech Russian Firms. Journal of product innovation management, 30(3), 545-559.

Scarbrough, H., Swan, J., Amaeshi, K., \& Briggs, T. (2013). Exploring the role of trust in the deal-making process for early-stage technology ventures. Entrepreneurship Theory and Practice, 37(5), 1203-1228.

Schraw, G., \& Dennison, R. S. (1994). Assessing metacognitive awareness. Contemporary educational psychology, 19(4), 460-475.

Shepherd, D. A. (2015). Party On! A call for entrepreneurship research that is more interactive, activity based, cognitively hot, compassionate, and prosocial. Journal of Business Venturing, 30(4), 489-507.

Shepherd, D. A., \& Patzelt, H. (2017). Researching the Generation, Refinement, and Exploitation of Potential Opportunities. In Trailblazing in Entrepreneurship (pp. 17-62). Springer International Publishing.

Simoni, M., Cautela, C., \& Zurlo, F. (2014). Product design strategies in technological shifts: An explorative study of Italian design-driven companies. Technovation, 34(11), 702-715.

St-Jean, E., \& Audet, J. (2012). The role of mentoring in the learning development of the novice entrepreneur. International Entrepreneurship and Management Journal, 8(1), 119-140.

Strike, V., \& Rerup, C. (2016). Mediated sensemaking. Academy of Management Journal 59(3), 880-905.

Teece, D. J. (2010). Business models, business strategy and innovation. Long range planning, 43(2), 172-194.

Vaghely, I. P., \& Julien, P. A. (2010). Are opportunities recognized or constructed?: An information perspective on entrepreneurial opportunity identification. Journal of Business Venturing, 25(1), 73-86. 
Venkataraman, S., Sarasvathy, S. D., Dew, N., \& Forster, W. R. (2012). Reflections on the 2010 AMR decade award: Whither the promise? Moving forward with entrepreneurship as a science of the artificial. Academy of Management Review, 37(1), 21-33.

Venkataraman, S., Sarasvathy, S. D., Dew, N., \& Forster, W. R. (2013). Of narratives and artifacts. Academy of Management Review, 38(1), 163-166.

Vlaar, P. W., van Fenema, P. C., \& Tiwari, V. (2008). Cocreating understanding and value in distributed work: How members of onsite and offshore vendor teams give, make, demand, and break sense. MIS quarterly, 32(2), 227-255.

Watson, T. J. (2011). Ethnography, reality, and truth: the vital need for studies of "how things work'in organizations and management. Journal of Management Studies, 48(1), 202-217.

Weick, K. E., Sutcliffe, K. M., \& Obstfeld, D. (2005). Organizing and the process of sensemaking. Organization science, 16(4), 409-421.

West, G. P. (2007). Collective cognition: When entrepreneurial teams, not individuals, make decisions. Entrepreneurship Theory and Practice, 31(1), 77-102.

Zott, C., \& Huy, Q. N. (2007). How entrepreneurs use symbolic management to acquire resources. Administrative Science Quarterly, 52(1), 70-105. 


\section{APPENDIX 1}

Exit interview template

Date:

Interviewer:

Participant:

Interview has been recorded.

Handwritten notes are available.

All topics are covered in interview.

Main points/observations:

1. How is your business idea (idea) now and how is this different from a year ago when you started, qualitative and quantitative, in as much detail as possible?

2. To what extent do you see yourself as an entrepreneur at this moment?

3. How is this different from a year ago when you started?

4. What is, according to you, a successful entrepreneur, and to what extent - and howdid this image change in the course of time?

5. What was your motivation to become an entrepreneur?

6. What did you expect when you came to the Venture Lab?

7. To what extent are those expectations fulfilled, and why/why not?

8. What things did you find easy in establishing the business?

9. What were your main challenges in the last year?

10. How did you solve these challenges (and to what extent did you seek advice (and from whom))?

11. To what extent did you meet your goals?

12. To what extent have you changed your goals or your vision during the last year, how and why (en in hoeverre hebben derden hierbij een rol gespeeld)?

13. To what extent have you changed your approach of doing business during the last year? How and why?

14. After you have participated in the Venture Lab program, what is your opinion about:

- the trainings, and why? 
- In general, what do you think of the usefulness or value of trainings for (starting) entrepreneurs?

- The networks, and why?

- In general, what do you think of the usefulness or value of networks for (starting) entrepreneurs?

- The coaching, and why?

- In general, what do you think of the usefulness or value of trainings for (starting) entrepreneurs?

- The panel presentations, and why?

- In general, what do you think of the usefulness or value of trainings for (starting) entrepreneurs?

- The physical infrastructure (building, offices, phones, printers etc.) and possibility for financial support or help to find this?

- In general, what do you think of the usefulness or value of infrastructural and/or financial support for (starting) entrepreneurs?

15. Which theories, models, or concepts that you have learned in VLT have been most useful to you and why? (please give examples)

16. What kind of (other) resources did you acquire?

17. To what extent did the services you got from the VLT (trainings, information, education, coaching, networks, panel presentations and everything else) influence you in the sense of making you:

a. More independent? Why?

b. More aware of (different) resources? Why?

c. More aware where to get those resources? Why?

d. More of the meaning or value of different resources, and why?

18. Wat do you think of the business model instrument (the business model canvas)? Why?

a. To what extent did your business model change, how and why?

19. Did you participate in business model generation workshop (3 days) or the business model canvas/generation training (one afternoon) and if yes why did you participate, and if you didn't participate, why not?

a. Wat was the goal of your participation? 
b. What did you gain from participating?

c. For what or how do you use the business model, and why?

20. Did you particpate in the synergie group, and if so why and what did you think of it?

a. What were your benefits from it, and why?

21. Did you particpate in the intervision group, and if so why and what did you think of it?

a. What were your benefits from it, and why?

22. What did you miss at the VLT in terms of services or other things?

23. What are your expectations for the future?

24. Would you recommend to participate in the VLT, and if so to whom?

25. Are you an alumnus, and if not, would you like to become one?

26. To what extent does your company use social media and how?

27. Could you tell us:

- Your educational background?

- Your professional background (work experience)?

- If your parents had/have entrepreneurship experience? 


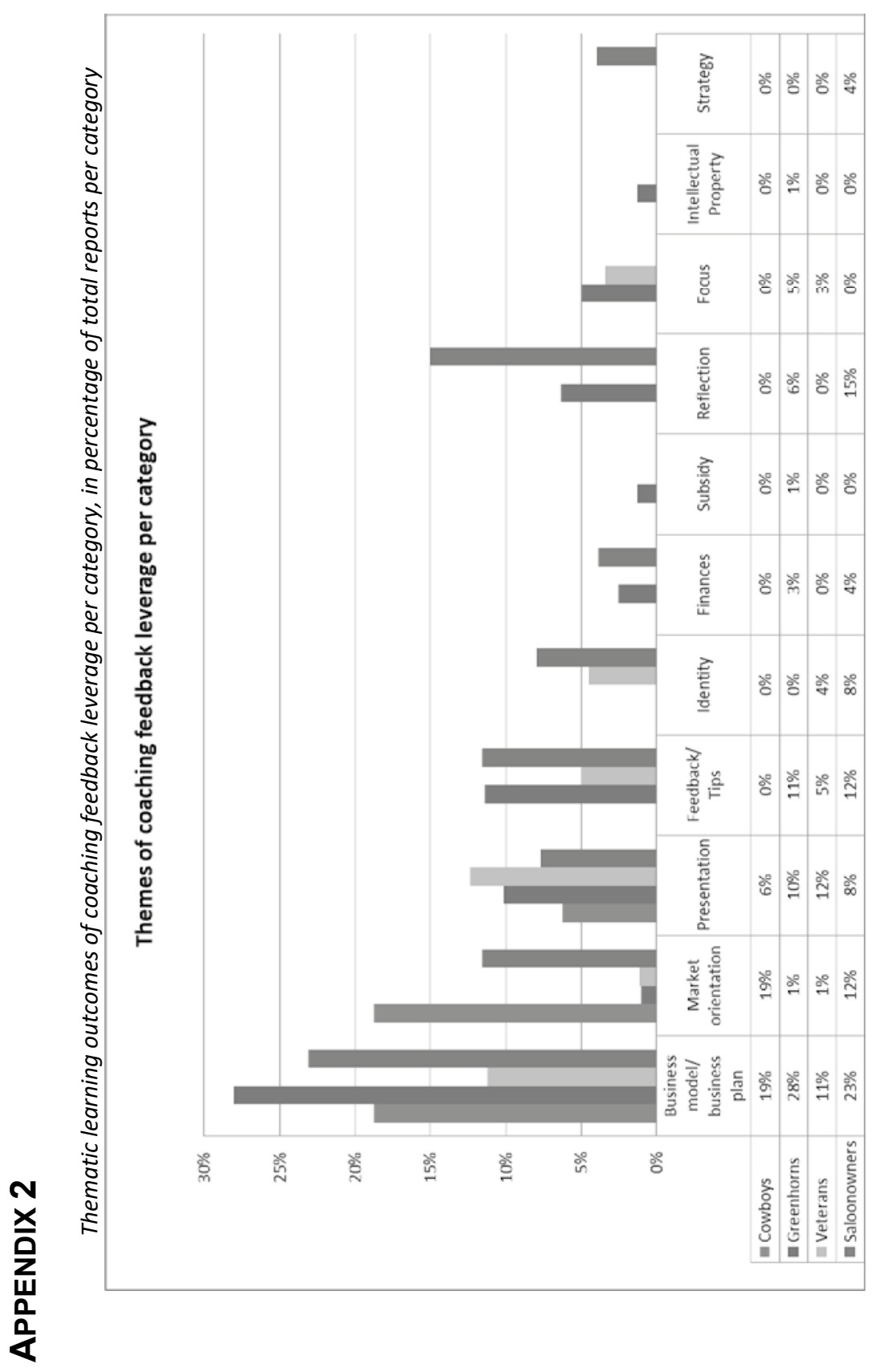




\section{EXECUTIVE SUMMARY}

This dissertation focuses on the topic of entrepreneurial cognitive development during business opportunity development. Business opportunity development takes place in a social context and is affected by the entrepreneur's (inter)action with relevant stakeholders (Clarke \& Cornelissen, 2011). Entrepreneurial researchers acknowledge the socially situated nature of entrepreneurial cognition (Mitchell et al., 2011). The research question central to this thesis is the following:

How do socially situated cognitive mechanisms affect entrepreneurial cognitive development during business opportunity development?

To answer this question, we undertook four empirical studies among entrepreneurs who participated in an institutional incubation/acceleration program.

In the first empirical study we explore the effect of two feedback mechanisms coaching and business panel presentation - on entrepreneurial cognitive development during opportunity development. To do so, we use the grounded theory approach and draw on data from weekly logbooks that the entrepreneurs kept. Our analysis yields a taxonomy in which we distinguish between four categories of entrepreneurs - based on the amount of prior entrepreneurial experience and the nature of their value proposition - which we label, respectively: 'Greenhorns', 'Cowboys', 'Trappers' and 'Saloon-owners'. The results show that panel feedback leads to learning of declarative knowledge among entrepreneurs, while coaching feedback fosters the development of reflective, metacognitive or 'deeper-level' entrepreneurial learning. We find that experienced entrepreneurs report this type of learning more often than novice entrepreneurs, while novice entrepreneurs report more learning of declarative knowledge. This study contributes to our understanding of feedback processes by the identification of complimentary effects of coaching and panel feedback mechanisms on the cognitive development of entrepreneurs.

In the second study, we examine the concept of sensebreaking and how third parties affect entrepreneurial cognitive development by means of sensebreaking. Sensebreaking is analysed in terms of its mechanisms of redirecting, reframing and questioning described by Vlaar et al. (2008). The results show that the mechanisms of redirecting and reframing affect entrepreneurial cognitive development differently. The 
results contribute to a more nuanced understanding of how different feedback mechanisms affect entrepreneurial cognition. Particularly, we found that reframing can prove valuable in creating 'cognitive space' for novel assessments and subsequent decision-making about the value of a resource. Meanwhile, redirecting facilitates incremental learning episodes, such as shifting the entrepreneur's attention to a different market or a novel partner in value creation.

In the third empirical study we explore the cognitive dimension of developing a market orientation. A market orientation is defined as sensemaking reflected in mental models used by entrepreneurs. Taking a grounded theory approach, we examine sensemaking processes of entrepreneurs who are engaged in solving problems encountered in the creation of (superior) customer value during business opportunity development. The results of our analysis yield six market-oriented mental models which are used by entrepreneurs. Our findings contribute to the conceptualization of the role and significance of stakeholders in solving problems related to creating (superior) customer value and underline the role of the social context in entrepreneurial sensemaking. In addition, the findings show that market-oriented mental models used by experienced entrepreneurs are more general and tacit in nature and reflect a more expertly skilled performance as described by Dreyfus \& Dreyfus (2005) and affects the way that experienced entrepreneurs could communicate their market-oriented problem to other members of the venture in the case of venture growth and the ensuing development of a venture-level market orientation.

The fourth empirical study contains an analysis of entrepreneurial sensemaking of the business model artifact. The business model is the organizational and financial 'architecture' of a business (Teece, 2010). Approached in its most abstract capacity of a model, the business model artifact can fulfill different purposes (Osterwalder et al., 2005). We distinguish between four purposes for which the business model artifact can be used, namely: understanding, communicating, analyzing and managing the firm's value creation logic. The results of our study show that entrepreneurs make sense of the business model artifact mainly for purposes of understanding and developing the venture's value creation logic. Our findings also show that entrepreneurs with a STEM education (in science, technology, engineering and mathematics) are twice as likely to use the communication purpose. The results of this study show that when used for its communicative purpose, the business model artifact provides entrepreneurs with a 
mechanism to involve relevant stakeholders in the creation of shared meaning, crucial for the successful creation of value (Clarke \& Cornelissen, 2014).

In the four empirical studies we identify different mechanisms - feedback mechanisms, sensebreaking mechanisms, market-oriented mental models and the business model artifact - which shape entrepreneurial sensemaking. Those mechanisms are socially situated, cognitive mechanisms by which third parties can shape entrepreneurial cognition. We conclude that the socially situated cognitive mechanisms we identify are enabling and facilitating instruments for the co-construction of entrepreneurial sensemaking, and ultimately for the development of entrepreneurial cognition during opportunity development.

With the results of the empirical studies in this dissertation we contribute to conceptualizing the co-constructed nature of entrepreneurial sensemaking. In particular, this dissertation contributes to empirical insights into the role of language in entrepreneurial cognitive development. Language provides a means to verbalize one's ideas and shapes entrepreneurial cognition (Clarke \& Cornelissen, 2014). The results of our research show language enables (meta-)cognitive development of entrepreneurs by means of socially situated cognitive mechanisms and demonstrate how entrepreneurs develop (meta-)cognitive skills through communicative interaction. Furthermore, this dissertation contributes methodically: By employing the diary method, we answer to Shepherd's (2015) call for methodological experimentation and modernisation in scientific entrepreneurship research.

On a practical level, entrepreneurs can leverage the insights from the empirical studies to reflect on and become (more) aware of being a particular 'type' of entrepreneur, particularly related to their previous entrepreneurial experience and the type of opportunity they are developing. For example, novice entrepreneurs can use cognitive development in terms of declarative knowledge provided by panel members to create focus in the business opportunity they are developing. In addition, trainings in using the business model artifact for different purposes provide entrepreneurs with an instrument to not only formulate and define, but also to analyze and communicate their opportunity's value creation logic. And by becoming aware and reflective of the stakeholder orientation (as part of the entrepreneur's market orientation) entrepreneurs can be more intentional about the set-up of a 'community of inquiry' (Shepherd \& Patzelt, 
2017) in which they can engage in thought exchange and leverage feedback from relevant stakeholders that help to shape the business opportunity.

Students and academic researchers can benefit from our findings as well. Corbett for example (2005) proposed that students in entrepreneurship courses should learn more about how to adapt their original ideas in reaction to changes suggested by potential customers and other actors in the market place. The socially situated cognitive mechanisms analyzed in this dissertation are suitable for stimulating and sustaining effective feedback-based (entrepreneurship) education because they can easily be integrated into experiential learning approaches which put emphasis on the development of soft skills, presentational skills and interpersonal communication, and which are considered important for higher education in general (Kolb \& Kolb, 2005) and in entrepreneurial thinking in particular (Krueger, 2007). The training in and subsequent conscious leverage of these mechanisms by individuals or in teams could be particularly valuable for multidisciplinary trajectories, not only in entrepreneurship education but also for multidisciplinary research projects.

This dissertation also provides useful insights for the design of business incubation/acceleration programs. Our research findings show that socially situated cognitive mechanisms represent enabling tools for actors in business support settings to 'steer', focus and target practice-based entrepreneurial learning. For example, policymakers can leverage the conceptualization of the process and outcomes of stakeholder interaction offered in the empirical studies in order to develop indicators for monitoring and evaluation of the efficient use of both coaching and panel interactions.

There are a few limitations to the research undertaken in this dissertation. Firstly, our research population consists of a relatively small number of entrepreneurs, a constraint often encountered in qualitative research; secondly, the diary data is a very 'raw' form of data because the entrepreneurs were free to write down thoughts about their development, only guided by the four 'topics' within each weekly diary, and subsequently entrepreneurs varied in terms of content and frequency of self-reporting. A third limitation is that the data stems from a sample of entrepreneurs who are part of a research incubation/acceleration program, entailing the possibility of a biased research population characteristically unwilling or unable to independently pursue business development but clever enough to seek help. However, this thesis contributes to the conceptualization of entrepreneurial cognitive development by identifying how socially 
situated cognitive mechanisms are relevant for the cognitive development of entrepreneurs. By doing so, this thesis provides 'legwork' by laying a basis for the design of further, explanatory studies in the area of entrepreneurial cognitive development.

In terms of avenues for future research, we discern the following topics worth investigating: For one, the employment of self-reports as a valuable method of data collection in entrepreneurship studies, in parallel to Foo, Uy \& Baron (2009) who advocate a more extensive use and critical evaluation of these methods. Second, future studies should focus on the relationship between socially situated cognitive mechanisms and venture survival, growth and employment generation - this which would yield valuable insights into the role that co-constructed sensemaking plays in the commercial success of entrepreneurial ventures. 


\section{SAMENVATTING}

Deze proefschrift richt zich op de cognitive ontwikkeling van ondernemers tijdens de ontwikkeling van hun bedrijfsidee. De ontwikkeling van bedrijfsideeën vindt plaats in een sociale context en wordt beïnvloed door de ondernemer (inter) actie met relevante stakeholders (Clarke \& Cornelissen, 2011). Ondernemersonderzoekers erkennen de maatschappelijk gelegenheid van ondernemerscognitie (Mitchell et al., 2011). De onderzoeksvraag van dit proefschrift is de volgende:

Hoe beïnvloeden sociaal gesitueerde cognitieve mechanismen de cognitieve ontwikkeling van ondernemers tijdens het ontwikkelen van een businessidee?

Om deze vraag te beantwoorden hebben we vier empirische studies ondernemers ondernomen die deelnamen aan een institutioneel incubatie-/ versnellingsprogramma.

De eerste empirische studie onderzoekt het effect van twee feedback mechanismen - coaching en business panel feedback - op de cognitieve ontwikkeling van onderenemers tijdens bedrijfsidee-ontwikkeling. Om dit te doen gebruiken we de grounded theory en halen de data uit wekelijkse logboeken die de ondernemers gedurende één jaar bijhielden. Uit de analyse komt een taxonomie naar voren waarin we onderscheid maken tussen vier categorieën ondernemers - gebaseerd op het aantal voorondergaande ondernemers ervaringen en de aard van hun waarde propositie - die we respectievelijk 'Greenhorns', 'Cowboys', 'Trappers' en 'Saloon-eigenaren' noemen. De resultaten laten zien dat panel feedback leidt tot het leren van declaratieve kennis onder ondernemers, terwijl coaching feedback de ontwikkeling van reflecterend, metacognitief of 'dieper niveau' leren bevordert. Er blijkt ook dat ervaren ondernemers metacognitieve ontwikkeling vaker rapporteren dan beginnende ondernemers, terwijl beginnende ondernemers meer het leren van declaratieve kennis rapporteren. Deze studie draagt bij tot het begrijpen van feedback-processen, door het identificeren van complimentaire effecten van coaching en panel feedback mechanismen op de cognitieve ontwikkeling van ondernemers.

In de tweede studie onderzoeken we het begrip sensebreaking en hoe derde partijen de cognitieve ontwikkeling van ondernemers beïnvloeden door middel van sensebreaking. Sensebreaking wordt geanalyseerd aan de hand van de mechanismen 
van redirecting, reframing en questioning beschreven door Vlaar et al. (2008). Uit de resultaten blijkt dat de mechanismen van redirecting en reframing de cognitieve ontwikkeling op verschillende manieren beïnvloeden. In het bijzonder hebben we geconstateerd dat reframing waardevol kan zijn bij het creëren van 'cognitieve ruimte' voor nieuwe beoordelingen en daaropvolgende besluitvorming over de waarde van een bron. Ondertussen vergemakkelijkt redirecting incrementeel leren, zoals de ondernemer's aandacht verleggen naar een andere markt of een nieuwe partner in waardecreatie. Deze resultaten dragen bij aan een meer genuanceerd begrip van hoe verschillende feedback-mechanismen ondernemerscognitie beïnvloeden.

In de derde empirische studie onderzoeken we de cognitieve dimensie van het ontwikkelen van een marktoriëntatie. Een marktoriëntatie wordt gedefinieerd als sensemaking dat in mentale modellen weerspiegelt die door ondernemers worden gebruikt. Met een grounded theorie-aanpak onderzoeken wij hoe ondernemers problemen oplossen tijdens het creëren van (superieure) klantwaarde en welke mentale modellen hierbij aangehaald worden. De resultaten van onze analyse laten zien dat ondernemers zes marktgerichte mentale modellen gebruiken tijdens het ontwikkelen van hun bedrijfsidee. Ook gebruiken ervaren ondernemers meer algemene modellen mentale schema's - dan ondernemers zonder ervaring. Onze bevindingen dragen bij tot de conceptualisering van de rol en betekenis van belanghebbenden bij het oplossen van problemen met betrekking tot het creëren van (superieure) klantwaarde en onderstrepen de rol van de sociale context in ondernemend sensemaking.

De vierde empirische studie bevat een analyse van sensemaking door ondernemers omtrent het business model artefact. Het business model is de organisatorische en financiële 'architectuur' van een bedrijf (Teece, 2010). Benaderd in zijn meest abstracte capaciteit als model, kan het business model artefact verschillende doeleinden vervullen (Osterwalder et al., 2005). We onderscheiden tussen vier doeleinden waarvoor het business model artefact kan worden gebruikt, namelijk: het begrijpen, communiceren, analyseren en beheren van de waardecreatie-logica van het bedrijf. Uit de resultaten van onze studie blijkt dat ondernemers het bedrijfsmodel artefact voornamelijk gebruiken om de waardecreatie-logica van de onderneming te begrijpen en te ontwikkelen. Uit onze bevindingen blijkt ook dat ondernemers met een STEMopleiding (in wetenschap, technologie, techniek en wiskunde) twee keer zo vaak het communicatie doeleinde gebruiken. De resultaten van deze studie tonen aan dat het bsuiness model artefact gebruikt wordt om relevante stakeholders te betrekken bij het 
creëren van gedeelde betekenis die van cruciaal belang is voor de succesvolle creatie van waarde.

In de vier empirische studies identificeren we verschillende mechanismen feedback mechanismen, sensebreaking mechanismen, marktgeoriënteerde mentale modellen en het business model artefact - wat vormt de ondernemende sensemaking. In die zin zijn deze mechanismen sociaal gesitueerde, cognitieve mechanismen waardoor derde partijen ondernemingscognitie kunnen vormen. Op basis van onze resultaten concluderen we dat de sociaal gesitueerde cognitieve mechanismen die we identificeren functioneren als instrumenten voor het samen construeren van sensemaking door ondernemers.

Met de resultaten van onze empirische studies dragen we bij aan het conceptualiseren van de co-constructie van sensemaking door ondernemers. In het bijzonder draagt deze proefschrift bij aan empirische inzichten in de rol van de taal in ondernemerscognitieve ontwikkeling. Taal biedt een middel om je ideeën te verbaliseren en ondernemerscognitie te vormen (Clarke \& Cornelissen, 2014). De resultaten van onze research laten zien hoe ondernemers (meta-) cognitieve vaardigheden ontwikkelen via communicatieve interactie en door middel van sociaal gesitueerde cognitieve mechanismen. Bovendien draagt deze proefschrift methodisch bij: Door gebruik te maken van de dagboek methode, beantwoorden we Shepherd's (2015) oproep tot methodologische experimentatie en modernisering in wetenschappelijk ondernemerschapsonderzoek.

Ondernemers kunnen de inzichten van de empirische studies inzetten om na te denken over en zich meer bewust te worden van het type 'ondernemer' dat zij zijn, met name in verband met hun eerdere ondernemingservaring en het soort idee wat zij ontwikkelen. Bijvoorbeel kunnen beginnende ondernemers baat hebben bij de ontwikkeling van declaratieve kennis door panel-leden, bijvoorbeeld op welke markt ze zich moeten richten en wie relevant is voor hun 'community of inquiry' (Shepherd \& Patzelt, 2017). Ook bieden trainingen in het gebruik van het businessmodel artefact voor verschillende doeleinden ondernemers een instrument om niet alleen de waardecreatielogica te begrijpen en te managen, maar ook om te analyseren en te communiceren. 
Studenten en academische onderzoekers kunnen ook profiteren van onze bevindingen. Corbett bijvoorbeeld (2005) stelde dat studenten in ondernemerscursussen meer zouden moeten leren hoe ze hun oorspronkelijke ideeën aanpassen in reactie op veranderingen die door potentiële klanten en andere actoren op de markt werden voorgesteld. De sociaal gesitueerde cognitieve mechanismen die in dit proefschrift worden geanalyseerd, zijn geschikt om (ondernemerschap) onderwijs te ontwikkelen waarin feedback een belangrijke rol speelt, of warain feedback-loops gemakkelijk kunnen worden geïntegreerd. Dit is bijzonder relevant voor experiential learning dat de nadruk legt op de ontwikkeling van zogenaamde 'soft skills', zoals presentatievaardigheden en interpersoonlijke communicatie. Deze laatste vaardigheden worden algemeen als belangrijk beschouwd in het hoger onderwijs (Kolb \& Kolb, 2005) maar met name ook in het ondernemersschapsonderwijs (Krueger, 2007). Het leren en daarop volgende bewuste gebruik van deze mechanismen door individuen of in teams kunnen voor multidisciplinaire trajecten bijzonder waardevol zijn, niet alleen dus in ondernemerschapsopleiding maar ook in multidisciplinaire onderzoeksprojecten.

Praktisch gezien bidet deze proefschrift empirische kennis over de rol van belanghebbenden bij de ontwikkeling van ondernemerscognitie. Deze inzichten kunnen gebruikt worden om het ontwerp van bedrijfsincubatie-/versnellingsprogramma's te optimaliseren. In het bijzonder bewijzen onze onderzoeksresultaten hoe cognitieve ontwikkeling van ondernemers kan worden bereikt door middel van gerichte feedback mechanismen die in dit proefschrift worden onderzocht. Daarnaast kunnen beleidsmakers de conceptualisering van het proces en de resultaten van de interactie tussen belanghebbenden uit de empirische studies van dit proefschrift gebruiken om indicatoren te ontwikkelen voor het monitoren en beoordelen van het efficiënt gebruik van bijvoorbeeld coaching en panel-interacties in programma's ter ondersteuning van (startende) ondernemers.

Er zijn een paar beperkingen op het onderzoek dat in dit proefschrift is ondernomen. Ten eerste bestaat onze onderzoekspopulatie uit een relatief klein aantal ondernemers, een beperking die vaak voorkomt bij kwalitatief onderzoek; Ten tweede zijn de dagboekgegevens een zeer 'ruwe' gegevensvorm omdat de ondernemers vrij waren om gedachten over hun ontwikkeling neer te schrijven, die alleen door de vier 'onderwerpen' worden geleid in elk wekelijks dagboek en vervolgens ondernemers variëren in termen van inhoud en frequentie Van zelfrapportage. Een derde beperking is dat de gegevens voortvloeien uit een steekproef ondernemers die deel uitmaken van 
een onderzoeksincubatie / versnellingsprogramma, waarbij de mogelijkheid bestaat dat een bevooroordeeld onderzoeksbevolking kenmerkend onwillig of niet in staat is om zelfstandig zelfstandig te volgen, maar slim genoeg om hulp te zoeken. Echter moet dit proefschrift vooral worden gezien als een 'eerste aanzet' die een basis legt voor het ontwerpen en uitvoeren van verdergaande studies op het gebied van cognitieve ontwikkeling van ondernemers.

In termen van mogelijkheden voor toekomstig onderzoek onderscheiden we de volgende onderwerpen die de moeite waard zijn om te onderzoeken: Ten eerste de inzet van zelfrapporten als een waardevolle methode voor gegevensverzameling in ondernemerschapstudies, parallel aan Foo, Uy \& Baron (2009) die pleiten voor een uitgebreider gebruik en kritische evaluatie van deze methoden. Ten tweede moeten toekomstige studies zich concentreren op de relatie tussen sociaal gesitueerde cognitieve mechanismen en het overleven, de groei en de gegenereerde werkgelegenheid van de onderzochte ondernemingen - dit zou waardevolle inzichten opleveren in de rol die co-constructed sensemaking speelt in het commercieel succes van ondernemingsondernemingen. 


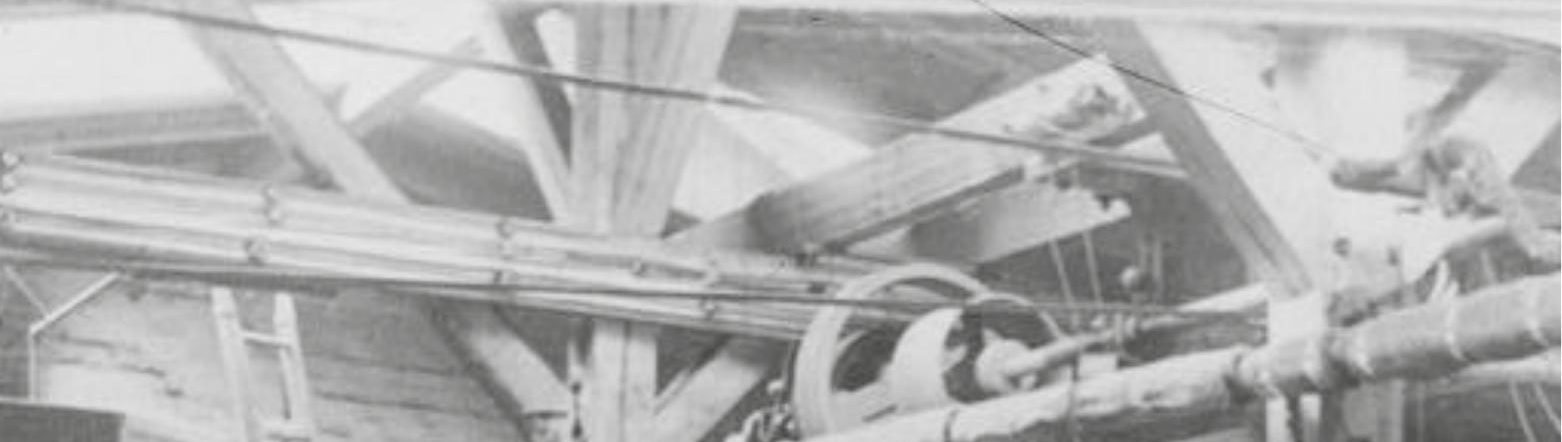

,
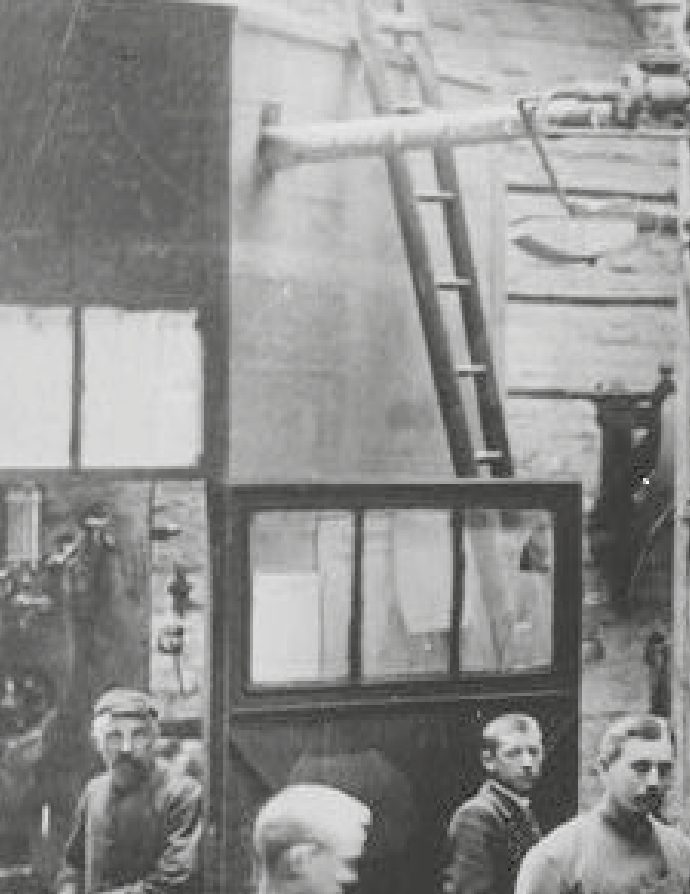

$1+\frac{6}{2}=$

$\sqrt{2}$

G

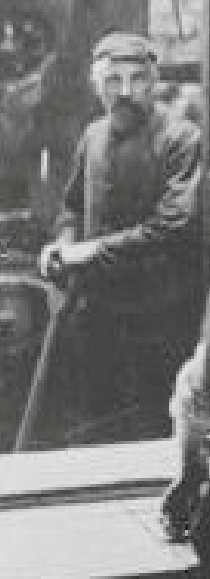

2.7. $\operatorname{lin}_{2}$

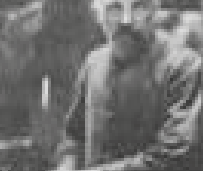
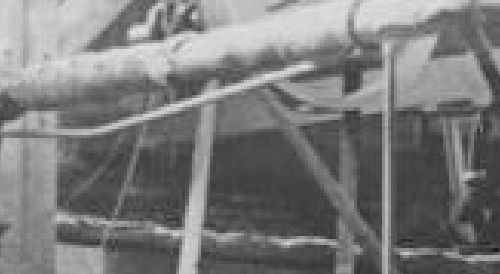

8

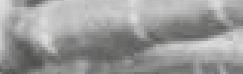
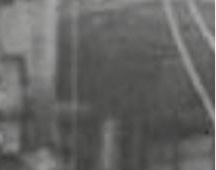